\title{
Applications of Cellular Components in Engineered Environments
}

\author{
Xiao Hu \\ West Virginia University, xihu@mix.wvu.edu
}

Follow this and additional works at: https://researchrepository.wvu.edu/etd

Part of the Biochemical and Biomolecular Engineering Commons

\section{Recommended Citation}

$\mathrm{Hu}$, Xiao, "Applications of Cellular Components in Engineered Environments" (2018). Graduate Theses, Dissertations, and Problem Reports. 3716.

https://researchrepository.wvu.edu/etd/3716

This Dissertation is protected by copyright and/or related rights. It has been brought to you by the The Research Repository @ WVU with permission from the rights-holder(s). You are free to use this Dissertation in any way that is permitted by the copyright and related rights legislation that applies to your use. For other uses you must obtain permission from the rights-holder(s) directly, unless additional rights are indicated by a Creative Commons license in the record and/ or on the work itself. This Dissertation has been accepted for inclusion in WVU Graduate Theses, Dissertations, and Problem Reports collection by an authorized administrator of The Research Repository @ WVU.

For more information, please contact researchrepository@mail.wvu.edu. 


\title{
Applications of Cellular Components in Engineered Environments
}

\author{
Xiao Hu \\ Dissertation submitted to the Benjamin M. Statler College of Engineering and Mineral \\ Resources at West Virginia University \\ in partial fulfillment of the requirements for the degree of
}

Doctor of Philosophy

in the

Chemical and Biomedical Department

\author{
Cerasela Zoica Dinu, Ph.D. Committee Chairperson \\ Charter Stinespring, Ph.D. \\ John Zondlo, Ph.D. \\ Xueyan Song, Ph.D. \\ Yon Rojanasakul, Ph.D.
}

Department of Chemical and Biomedical Engineering

Morgantown, West Virginia

2018

Keywords: Microtubule, Kinesin, Atomic Force Microscopy, Biosensor

Copyright 2018 Xiao Hu 


\title{
ABSTRACT \\ Applications of Cellular Components in \\ Engineered Environments
}

\begin{abstract}
Xiao Hu
Manipulation of cellular components in synthetic environment has attracted interest for applications ranging from sensors to nanelectronics and from catalysis to biomedical devices. In such applications, biological-based processes such as specific recognition and self-assembly direct the formation of hierarchical structures, all at minimum energetic costs, with high efficiency and reliability.
\end{abstract}

Among such cellular machines, microtubules and kinesins have attracted special interest. Inside the cells, microtubules are giving structural integrity while serving as regular and uniform tracks for transport of vesicles or organelles. Kinesin uses a microtubule track in vivo to progress to specific locations with processive and coordinated steps, all under the transformation of the chemical cycle of adenosine triphosphate (ATP) into mechanical work. Manipulation of microtubules and kinesins in vitro has been implemented for the transport of synthetic cargos like beads, nanoparticles or quantum dots to specific locations for molecular detection or diagnosis. However, in such applications, individual separation of a microtubule or kinesin-based complex, or parallel and yet individual sustainability and attainability of such complexes, could not be achieved. Main challenges consisted of biological components susceptibility to experimental conditions or lack of stability and complexity of tasks to be achieved under ambient temperature and pressure conditions.

This thesis presents novel concepts and implementations of such cellular components in engineered environments. From studying single molecule assembly forces using atomic force microscopy approaches, to synthesizing novel self-assembled hybrid materials combining advantages of organic and inorganic components, and from application of cellular components in nanoelectronics or as the next generation tools for single molecule printing, the work included offers viable solutions for emerging science and engineering concepts that promote natural-based processes for synthetic applications. 
Dedication

To my family. 


\section{Acknowledgement}

For the past five years during my Ph.D. program training, there are so many people that have earned my gratitude for their kind help and support.

First, I would like to express my sincere gratitude and special appreciation to my advisor Professor Cerasela Zoica Dinu for her continuous advice, assistance and support for my Ph.D study. Her guidance helped me in all the time of research and writing this thesis. Her support, help and encouragement allows me to grow as a research scientist. She inspired me by her hardworking, passionate and conscientious attitude on the personal level. My sincere thanks also goes to the rest of my thesis committee members, Professor Charter Stinespring, Professor John Zondlo, Professor Xueyan Song, and Professor Yon Rojanasakul, for their insightful comments on my research. I also want to thank staff scientists in WVU shared research facility, without their kind help and professional suggestions some of my work would not occur.

I would like to thank all the other members in Prof. Dinu's lab and also the other faculty and stuff in the Chemical and Biomedical Engineering Department at WVU. This more than 5 years Ph.D. study experience at WVU means a lot to my life and has an important impact on my career.

Last but not the least, I would like to thank my friends and family who have always support me. 


\section{Table of Contents}

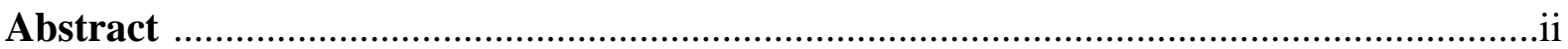

Dedication

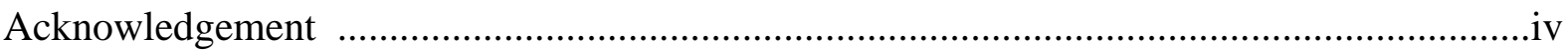

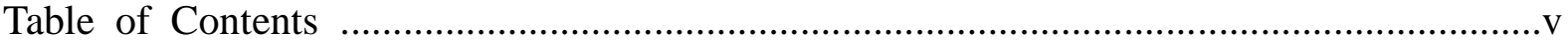

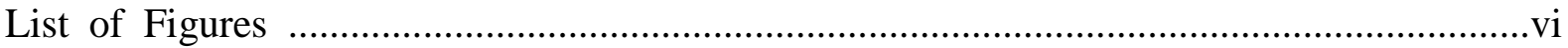

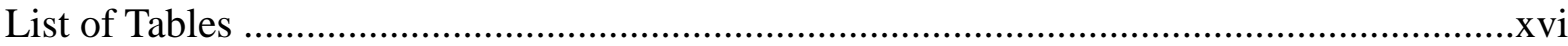

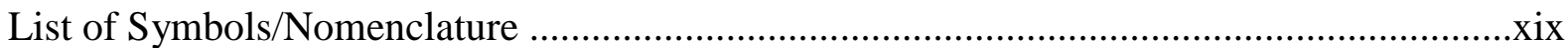

Chapter 1: Development of an Atomic Force Microscopy-based method for analysis of affinities between specific biological ligands 1

Chapter 2: Bio-pen for direct writing of single molecules on user-functionalized surfaces. .25

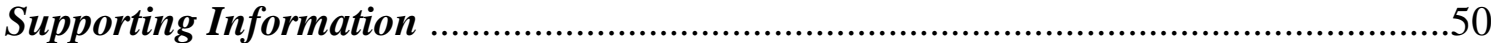

Chapter 3: Protein self-assembly onto nanodots leads to formation of conductive bio-based hybrids 58

Supporting Information .88

Chapter 4: Biological self-assembly and recognition used to synthesize and surface guide next generation of hybrid materials

Supporting Information 122

Chapter 5: Biomolecular interfaces based on self-assembly and self-recognition form biosensors capable to record single molecule binding and release 127

Supporting Informaiton .161

Conclusions 165 


\section{List of Figures}

\section{Chapter 1: Development of an Atomic Force Microscopy-based method for analysis of affinities between specific biological ligands}

Figure 1: Concept drawing of surface functionalization and contact mode analysis performed using Atomic Force Microscopy (AFM). (a) Functionalization of streptavidin protein to the glass surfaces; functionalization was performed using covalent binding. (b) Functionalization of anti-streptavidin antibody or biotin to the AFM tip. Schematic representation of the rupture force between streptavidin and its ligands, i.e., anti-streptavidin antibodies (c), or biotin used as control (d) respectively.

Figure 2: Binding forces between streptavidin and its ligands, namely anti-streptavidin antibodies and biotin used as control. (a) Contact mode force map analysis of the rupture force between representative anti-streptavidin antibody-coated tip and streptavidin-coated glass surface. (b) Contact mode force map analysis of the rupture force between representative biotin-coated tip and streptavidin-coated glass surface. The colored squares are associated with different values of the rupture forces being measured, with the darker representing smaller and brighter colors representing larger rupture forces respectively. (c) Representative distribution of the rupture forces between streptavidin-coated glass surface and anti-streptavidin antibody-coated tip. (d) Representative distribution of the rupture forces between streptavidin-coated glass surface and the biotin-coated tip. The distributions were obtained from independent force maps, with the two force maps for the individual ligands being analyzed being also representatives of all parallel experiments $(n=12)$.

Figure 3: Loading rate dependence on the rupture force in the unbinding process of the (a) anti-streptavidin antibody-streptavidin; and (b) biotin-streptavidin respectively. The $\mathrm{x}$ axis is the logarithm of the loading rate while the $\mathrm{y}$ axis represents the rupture force; 12 independent force maps were obtained for each of the protein-ligand being investigated.

Figure 4: Histogram of the average rupture force between streptavidin and its ligands, with at least 240 independent force maps analyses being performed for each of the complexes being investigated. Control I and control II represents the average rupture force between biotin- and anti-streptavidin antibody-coated tip and the glass respectively (with at least 60 
independent force maps for each of the controls). A Student T-test was carried out with the significance level of $\mathrm{p}^{*}<0.05 ;(*)$ indicates significance between individual complexes and the control while $(* * *)$ indicates significant from the different complexes. 


\section{Chapter 2: Bio-pen for direct writing of single molecules on user-functionalized surfaces}

Figure 1: (a) The structure of the proposed kinesin-inked microtubule pen and its in vitro optical representation; microtubule is shown in red and kinesin is shown in green. All green signal was increased by the software to give better visibility of the kinesin. (b) Schematic of the contact mode AFM analysis of the kinesin-inked microtubule pen. (c) Representative morphology of the kinesin-inked microtubule pen. The beads represent individual kinesin molecules bound to the surface of an immobilized microtubule, under the chemical energy of AMPPNP. (d) Representative morphology of a microtubule used as control. (e) Height profile analysis help evaluate the distributions of kinesin inks onto a microtubule pen. Upper image: readings are done across the sample to evaluate the diameter of the individual bound kinesins. Lower image: readings follow sample's profile and help identify the change in the microtubule local diameter when a bound kinesin is encountered. (f) Average diameters of the kinesin-inked microtubule pens and of control microtubules respectively. Student's T-test considered significance level of $* p<0.05$.

Figure 2: a) Schematic drawing of the AFM tip functionalization with anti-tubulin antibodies for controlled affixing of the kinesin-inked pen. b) Left upper: optical microscopy image showing the microtubule pen immobilized onto the tip through tubulin-anti-tubulin specific interactions. Left lower: optical microscopy image showing green kinesin inks onto the immobilized microtubule. The white dashed lines depict tip's profile. Right: representative fluorescence image of the kinesin-microtubule pen affixed at the tip formed from the merged images of red (microtubule) and green (labeled kinesin) signals. All green signal was increased by the software to give better visibility of the kinesin. c) Representative fluorescent images of the kinesin ink (green) moving onto an affixed microtubule pen (red). The distance traveled allows for a measurable speed of about $3 \mu \mathrm{m} / \mathrm{s}$. All green signal was increased by the software to give better visibility of the kinesin. d) The relationship between the visually observed length and the actual length that kinesin could travel onto an affixed pen. The different orientations of the affixed microtubule led to the different angles $(\theta)$ of observation.

Figure 3: a) Schematic of the glass multi-step functionalization process through zero-length chemistry and antibody binding through APTES-based reaction. b) Representative AFM morphology of anti-kinesin antibodies coated surfaces and the statistical 
variation in such height distribution (as depicted on the red line). c) Schematic of single kinesin molecules written by the affixed microtubule pen. d) Representative AFM morphology and distribution of single kinesin molecules written by the affixed microtubule pen and the statistical variation in the height distribution of kinesin immobilized onto anti-kinesin antibody (as depicted on the red line). The possible geometries and resulting anisotropies are discussed relative to the individual molecule packing at the user-functionalized interface.

Figure 4: a) The statistical variation in the height distribution of kinesin immobilized onto anti-kinesin antibody and control of height distribution of anti-kinesin antibody. The possible geometries and resulting anisotropies are discussed relative to the individual molecule packing at the user-functionalized interface. b) Kinesin walks on the different filaments of the microtubule and written onto anti-kinesin antibody functionalized surfaces (left) to be leading to circular morphological patterns (right). 


\section{Chapter 3: Protein self-assembly onto nanodots leads to formation of conductive bio-based hybrids}

Figure 1: Surface characterization of the S-doped C-dots. a) Representative Atomic Force Microscopy (AFM) analyses that allow for individual or conglomerates of nanodots visualization. AFM insert in the scale of $1 \mu \mathrm{m} \times 1 \mu \mathrm{m}$ allows for particle size analyses. b) The diameter distribution of the S-doped C-dots as resulted from AFM evaluations. c) Fourier Transform Infrared Spectroscopy (FTIR) of the S-doped C-dots allows for identification of surface functional groups, while Energy Dispersive X-Ray (EDX) analysis (d) reveals the elemental composition of the S-doped C-dots. e) The X-ray Photoelectron Spectroscopy (XPS) survey graph of the S-doped C-dots. f) High-resolution $S_{2 p}$ XPS spectra of the S-doped C-dots. g) High-resolution $\mathrm{C}_{1 \mathrm{~s}}$ XPS spectra of the S-doped C-dots.

Figure 2: a) Representative schematic of the chitosan-based membrane formation at the gold electrode interface. b) The reduction reaction of the methylene blue (MB) onto the electrode surface. c) Differential Pulse Voltammetry (DPV) graph of the MB on 1) chitosan/gold electrode (red), 2) S-doped C-dots/chitosan/gold electrode (green).

Figure 3: a) Average diameter of microtubules and the S-doped C-dots hybridized microtubules as measured using cross-sectional analyses. b) The diameter distribution of 72 individual microtubules collected from 20 independent images. c) The diameter distribution (as measured using cross-sectional analyses) of S-doped C-dots hybridized microtubules and collected from 24 independent AFM images that included 93 individual microtubules. d) Different orientations or combination of such orientations of the APTES molecules on the mica surface lead to different geometries and size distribution of the microtubules.

Figure 4: a) Representative morphology AFM analyses of microtubules after crosslinking with glutaraldehyde. b) Representative morphology AFM analyses of S-doped C-dots hybridized microtubules after crosslinking with glutaradehyde. Reduced stability was observed for the microtubules not crosslinking with glutaraldehyde (c) while the morphologies of the hybrid microtubules was not affected by the lack of the crosslinking agent (d). e) Height distribution of the microtubule (along the red line 1 in Figure 4a; cross-sectional analyses) or S-doped C-dots hybridized microtubules (along the red line 2 in Figure $4 \mathbf{b}$; cross-sectional analyses $)$ with the blue square representing the start point $(0.0 \mu \mathrm{m})$. 
The line followed the sample morphology.

Figure 5: a) Electrochemical impedance spectrum (EIS) graph of the electrode. 1. Bare gold electrode. 2. S-doped C-dots/Chitosan/Gold electrode. 3. Chitosan/Gold electrode. 4. S-doped C-dots-microtubule (MTs)/Chitosan/Gold electrode. 5. Microtubule (MTs)/Chitosan/Gold electrode. b) Differential pulse voltammetry (DPV) graph of the methylene blue on different electrode. The color-coding is similar to what described in a). c) Scheme of microtubule polymerization from free tubulin under the chemical energy of guanosine triphosphate. d) Scheme of the polymerization process of the S-doped C-dots hybrid microtubule; the schematic is not to scale. 


\section{Chapter 4: Biological self-assembly and recognition used to synthesize and surface guide next generation of hybrid materials}

Figure 1: (a) Scheme of tubulin attachment onto user-synthesized S-doped C-nanodots (S-doped C-dots). (b) UV absorbance of S-doped C-dots and tubulin-S-doped C-dot conjugates respectively. (c) Circular dichroism (CD) graph of tubulin, S-doped C-dots and tubulin-S-doped C-dot conjugates respectively.

Figure 2: (a) Representative atomic force microscopy-based (AFM) morphology of S-doped C-dot hybrid after crosslinking with glutaraldehyde. (b) Representative morphology AFM analyses of microtubule after crosslinking with glutaradehyde (control). Schematic representation of the AFM height distribution analysis along (c) and across (d) a hybrid. (e) Diameter distributions of 83 individual microtubules and 68 hybrids collected from 25 and 20 independent images respectively. (f) Height distributions of 211 individual microtubules and 181 hybrids collected from 25 and 20 independent videos respectively.

Figure 3: (a) Scheme of the kinesin-microtubule/hybrid binding in the presence of AMP-PNP. Representative AFM images of kinesin-hybrid (b) and kinesin-microtubule used as control (c). The line was chosen to follow the morphology of the sample for height analysis. d) The diameter distribution of 68 individual hybrids, 66 individual kinesin-hybrids measured on the kinesin and 80 individual kinesin-hybrid measured randomly, all collected from 20 and 22 independent images respectively. e) The diameter distribution of 83 individual microtubules, 70 individual kinesin-microtubule measured on the kinesin dot itself and 82 individual kinesin-microtubule measured randomly and collected from 25 and 23 independent images respectively. (f) Possible orientations of kinesin onto microtubule/hybrid.

Figure 4: (a) Schematic of the stepping assay. Briefly, a user-made flow cell was coated with anti-tubulin antibody for protein specific interactions. The stepping activity of the kinesin was observed using fluorescent microscopy, all under provided chemical ATP energy. (b) Fluorescent images of kinesin-hybrids at different time points; analysis (white line) show kinesin (green) ability to step on hybrids (red) as shown by the distance displacement. (c) Fluorescent images of kinesin-microtubule at different time (controls); again, analysis (white line) show kinesin (green) ability to step on control microtubule (red) as recorded by distance displacement. 
Figure 5: (a) Schematic of the gliding assay; casein was used as a blocking molecule to prevent non-specific binding of microtubules or hybrids. AMP-PNP bound GFP-kinesin attached to the flow cell was used to propel microtubules or hybrids when the AMP-PNP energy source was exchanged for ATP. (b) Fluorescent images of hybrids glided onto surface immobilized kinesin. (c) Fluorescent images of microtubules glided onto surface immobilized kinesin. 


\section{Chapter 5: Biomolecular interfaces based on self-assembly and self-recognition form biosensors capable to record single molecule binding and release}

Figure 1: a) Schematic illustration of kinesin functionalization with ferrocene monocarboxylic acid (FCA). b) The cyclic voltammetry (CV) of unlabeled kinesin (simply called kinesin), FCA-kinesin conjugates and free FCA.

Figure 2: a) Schematic illustration of FCA-kinesin conjugate binding to a microtubule (MT) to form a FCA-kinesin-MT assembly in the presence of adenylyl-imidodiphosphate (AMP-PNP). b) The assembly was immobilized onto an anti-tubulin antibody functionalized electrode. Prior immobilization, surface functionalization of the electrode with self-assembly monolayers of 11-mercaptoundecanoic acid (MUA) and bovine serum albumin (BSA) aimed to reduce non-specific binding, was used. c) The CV analysis of different concentrations of FCA-kinesin-MT assembly immobilized at the modified electrode interface. d) Electrochemical impedance spectrum (EIS) at the functionalized electrode interface. Black: bare gold electrode (Au). Pink: MUA/Au. Red: Anti-tubulin Antibody/MUA/Au. Blue: Anti-tubulin Antibody (BSA)/MUA/Au. Green: FCA-kinesin-MT/Anti-tubulin Antibody (BSA)/MUA/Au. e) $\mathrm{CV}$ of background (black; obtained at the modified electrode (Anti-tubulin Antibody (BSA)/MUA/Au) interface), FCA-kinesin-MT (red) at the modified electrode interface, free FCA (blue) at the anti-tubulin antibody functionalized electrode interface and free FCA-kinesin control (pink) at the anti-tubulin antibody functionalized electrode (Anti-tubulin Antibody (BSA)/MUA/Au) interface respectively.

Figure 3: a) The relationship between the anodic peak currents and the concentrations of the FCA-kinesin-MT assemblies immobilized onto the functionalized electrode. Inset: The linear relationship between the peak currents and the concentrations of the FCA-kinesin-MT assemblies immobilized onto the electrode. b) The relationship of the charge involved in the redox reaction $(\mathrm{Q})$ at the modified electrode and the concentrations of the FCA-kinesin-MT assemblies. Inset: The linear relationship between the c/Q and the concentrations of FCA-kinesin. All experiments were performed at a scan rate of $50 \mathrm{mV} / \mathrm{s}$.

Figure 4: a) CV of FCA-kinesin at modified electrode under different scan rates (6-300 $\mathrm{mV} / \mathrm{s}$ ). The concentration of the FCA-kinesin was kept constant at $42.2 \mathrm{nM}$, which represented that concentration that limits the saturation sites of the MTs. b) The linear 
relationship between the scan rates and the peak currents of the FCA-kinesin conjugates. c) Laviron's plots. All experiments were carried at modified electrode (Au/MUA/Anti-tubulin Antibody (BSA)/MT) in BRB80 buffer (pH 7.1) with $10 \mu \mathrm{M}$ taxol.

Figure 5: a) Schematic illustration of the FCA-kinesin conjugates stepping of the MT imoobilized at the electrode interface upon the addition of ATP. b) CV of FCA-kinesin conjugate at the modified electrode modified electrode (Au/MUA/Anti-tubulin Antibody/MT) after adding $250 \mu \mathrm{M}$ ATP. 


\section{List of Tables}

Chapter 1: Development of an Atomic Force Microscopy-based method for analysis of affinities between specific biological ligands

Table 1: Bell Model Parameters from F vs $\ln \left(\mathrm{L}_{\mathrm{r}}\right)$ relationship 
Chapter 3: Protein self-assembly onto nanodots leads to formation of conductive bio-based hybrids

Table 1. Percentage distribution of the elements present in the user-synthesized S-doped C-dots. 
Chapter 4: Biological self-assembly and recognition used to synthesize and surface guide next generation of hybrid materials

Table 1: CD characterization of tubulin protein and tubulin-S-doped C-dot conjugates. 


\section{List of Symbols/Nomenclature}

1. AFM: Atomic force microscopy

2. EDX: Energy dispersive X-ray spectroscopy

3. SEM: Scanning Electron Microscopy

4. EDC: 1-Ethyl-3-(3-dimethylaminopropyl)-carbodiimide

5. NHS: N-hydroxysuccinimide

6. PBS: Phosphate buffered saline

7. MES: 2-(N-morpholino)ethanesulfonic acid

8. MT: Microtubule

9. CV: Cyclic voltammetry

10. DPV: Differential pulse voltammetry

11. CD: Circular dichroism

12. UV: Ultra violet

13. MB: Methylene blue

14. EIS: Electrochemical impedance spectrum

15. ATP: Adenosine triphosphate

16. GTP: Guanosine triphosphate

17. AMP-PNP: Adenylyl-imidodiphosphate

18. FTIR: Fourier transform infrared spectroscopy

19. XPS: X-ray photoelectron spectroscopy

20. GFP: Green fluorescent protein

21. FCA: ferrocene carboxylic acid 


\title{
Chapter 1: Development of an Atomic Force Microscopy-based method for analysis of affinities between specific biological ligands
}

\begin{abstract}
In the cell, protein-ligand recognition involves association and disscociation processes controlled by the affinity of the two binding partners and chemical harvesting of adnosine triphosphate energy. Fundamental knowledge of selected recognition events is currently translated in synthetic environment for biosensors, immunoassays and diagnosis applications, or for pharmaceutical development. However, in order to advance such fields, one needs to determine the lifetime and binding efficiency of the two partners, as well as the complex' energy landscape parameters. We employed contact mode atomic force microscopy to evaluate the association and dissociation events between the streptavidin protein and its anti-streptavidin antibody ligand, currently used for nucleotide array, ELISA, and flow cytometry applications, just to name a few. Using biotin as control, our analysis helped characterize and differentiate multi- and single bonds of different strengths and associated energy landscapes to determine protein-ligand structural arrangement at nanointerfaces and how these depend on the specificity of the ligand-recognition reaction. Further, our results revealed that understanding the importance of the rupture forces between protein and its ligand could serve as the first step to protect on-off switches for biomedical research applications where specificity and selectivity are foremost sought.
\end{abstract}




\section{Introduction}

Biological world maintains structure and function through operative molecular interactions. For instance, the specific interactions and high recognition affinity between antibodies and protein antigens contribute to pathogen and their product recognition to ultimately provide immune protection ${ }^{1,2}$. Accordingly, protein antigen and antibody binding affinity and energetics are becoming a prominent area in therapeutics where ligand specificity influences humoral immunity ${ }^{3-6}$.

Fundamental knowledge of molecular interactions binding affinity and strength ${ }^{7}$ was gained mostly by studying their in situ characteristics using techniques relying on optical ${ }^{8}$ and magnetic tweezers ${ }^{9}$, or impedance spectroscopy ${ }^{10}$. For instance, Sischka et al., studied the dynamic translocation of ligand-complexed DNA through solid-state nanopores using optical tweezers ${ }^{11}$, Liebesny et al., determined the number of proteins bound non-specifically to DNA using magnetic tweezers ${ }^{12}$, while Duan et al., showed how impedance spectroscopy could be used to study the formation of a silicon nanowire field-effect transistor capable to measure protein-ligand binding affinities and kinetics with sensitivities down to femtomolar concentrations ${ }^{13}$. However, the implementation of such studies is currently hindered by either optical perturbations and how these affect the performance of the tweezers ${ }^{14,15}$, or the complexity and time scale of sample preparation and sample storage ${ }^{15}$, and the reduced detection capability of the instrument and how instrument's overheating influences the measurement process ${ }^{15}$ especially for protein-ligand pairs known to be heat sensitive ${ }^{16}$.

Atomic Force Microscopy (AFM) was recently implemented to circumvent some of these drawbacks; the technology does not require a specific or tedious pretreatment of the samples, is user and environmentally friendly, and allows for nano-resolution while depicting the exquisite surface structure of the samples being investigated ${ }^{17}$. As such, AFM was employed to visualize the surface architecture of Corynebacterium glutamicum with the analyses revealing an inner layer with about $11 \mathrm{~nm}$ periodicity in the structure of the individual cell ${ }^{18}$. The interaction parameters, such as the rupture force and dissociation constant, energy landscape parameters or energy bond and energy barrier width of the biotin and streptavidin bond were also quantified using $\mathrm{AFM}^{19}$; biotin and streptavidin are known to form the strongest non-covalent interaction in the biological world. Complementary, AFM analysis of the newly identified protein Gli349 and sialyllactose molecules revealed an unbinding force of around $70 \mathrm{pN}$; the Gli family is involved in the gliding of the bacteria Mycoplasma $^{20}$. Similarly, Ge et al., used AFM to study the specific interaction between 
bivalent aptamer $\mathrm{Bi}-8 \mathrm{~S}$ and investigated the unbinding dynamics and dissociation energy landscape of their multivalent interactions ${ }^{21}$; the aptamer is a class of molecular probes used for protein recognition, detection, and inhibition.

Translating and implementing the knowledge of binding affinity and strength led to the development of immune deficiency syndrome (AIDS) ${ }^{22}$ and cancer $^{23}$ detection assays. Complementary, studying the interaction of two complementary or partly matched single strands of DNA molecules helped identify DNA mutations ${ }^{24}$ or damages ${ }^{25}$, while self-assembly monolayer technology relying on binding and recognition at electrode interfaces is currently used in controlling deposition of nanostructures ${ }^{26}$ or biosensor's sensitivity ${ }^{27}$. However, there are still fundamental questions to be answered before further implementation of biomolecular interactions in synthetic assays is to be realized. In particular, scientific endeavor is required to investigate how single molecular scale interactions at the protein-ligand binding pocket site can be distinguished from non-specific interactions such as binding at intermediate positions. Lack of specificity is associated with weaker interactions that could lead to both low sensitivity and low detection capabilities in synthetic assays such as ELISA ${ }^{28}$ or immunodiagnostic ${ }^{29-32}$. Such endeavors require understanding of the basis for protein-ligand binding as well as the energetics associated with protein-ligand individual complexes formation.

To help answer these questions, we formulated a quantitative method that allowed for the ranking selection of unbinding forces between streptavidin and its ligand, namely anti-streptavidin antibody, all under known mechanical stress. By controlling the specificity of binding and limiting non-specific interactions, we showed through multi-Gaussian fitting that fine tuning of the protein-ligand interactions can help identify the disposition of protein binding site, distinguish the protein arrangement at interfaces and its influence on the energy landscape, as well as shed light into the characteristics of the protein-ligand recognition event and the association and dissociation events. Unraveling such events allowed analysis of ligand-breaking maps, all under a known mechanical force, to be used for a variety of applications from ELISA ${ }^{28}$ to immuno-diagnosis or -reagent analysis ${ }^{22,23}$. 


\section{Materials and Methods}

\section{Functionalization of the Atomic Force Microscope (AFM) tips}

TR-400PB tips (purchased from Asylum Research, USA) were functionalized with either biotin or anti-streptavidin antibodies. Briefly, the tips were first cleaned using a three steps cleaning protocol which consisted of first immersing them into $1 \mathrm{~mL}$ of deionized water for $10 \mathrm{~min}$, secondly into acetone (Fisher Scientific, USA) for another $10 \mathrm{~min}$, and lastly, in deionized water for 10 more minutes. Subsequently, the tips were dried under air, exposed to ultraviolet (UV) light for $30 \mathrm{~min}$, rinsed again with $100 \mathrm{mM} \mathrm{pH} 7.0$ phosphate buffered saline (PBS, made from mono-potassium phosphate, dipotassium hydrogen phosphate and sodium chloride, all reagents purchased from Fisher Scientific, USA) and incubated in solution of either $1 \mathrm{mg} / \mathrm{mL}$ biotin (Sigma, USA) or anti-streptavidin antibodies (Sigma, USA) for $3 \mathrm{~h}$ at room temperature. After incubation, the tips were rinsed in PBS to remove loosely bound ligands and used immediately.

\section{Cleaning of glass substrates}

Glass slides ( $\mathrm{d}=25 \mathrm{~mm}$, Corning, USA) were ultrasonicated in deionized water, $99 \%$ alcohol (Fisher Scientific, USA) and again in deionized water for $30 \mathrm{~min}$ each. Subsequently the slides were dried under vacuum for 1 day and before use exposed to UV light for $30 \mathrm{~min}$.

\section{Protein functionalization of the glass substrates}

Streptavidin was covalently attached to the cleaned glass slides using established methods ${ }^{33}$. Briefly, glass slide was immersed into a Piranha solution $(96.4 \%$ sulfuric acid, $\mathrm{H}_{2} \mathrm{SO}_{4}$, Fisher Scientific, USA) and $30 \%$ hydrogen peroxide $\left(\mathrm{H}_{2} \mathrm{O}_{2}\right.$, Fisher Scientific, USA) in a 3:1 volume ratio, and heated to $120^{\circ} \mathrm{C}$ for $10 \mathrm{~min}$. After exposure to Piranha, the slide was thoroughly washed with deionized water and dried under vacuum for 1 day. Upon drying, the slide was immersed in $1 \mathrm{ml}$ of 5\% 3-aminopropyltriethoxysilane (APTS; Fisher Scientific, USA), 99.5\% toluene solution (Fisher Scientific, USA) and incubated at room temperature for $1 \mathrm{~h}$. Upon time elapse, the slide was again washed thoroughly with deionized water, toluene and deionized water and subsequently immersed into $1 \mathrm{~mL} \mathrm{5 \%} \mathrm{glutaraldehyde} \mathrm{(Acros}$ Organics, USA) in $0.2 \mathrm{M} \mathrm{pH} \mathrm{9.0} \mathrm{Tris-buffered} \mathrm{saline} \mathrm{(TBS,} \mathrm{made} \mathrm{from} \mathrm{tris} \mathrm{and} \mathrm{hydrochloric}$ acid, reagents purchased from Fisher Scientific, USA) at room temperature, for $1 \mathrm{~h}$ with shaking at $200 \mathrm{rpm}$. Lastly, the slides were extensively rinsed with TBS, activated for 15 min in a $1 \mathrm{ml}$ solution of $\mathrm{pH} 4.7$ of 2-(N-morpholino)ethanesulfonic acid (MES, Fisher Scientific, 
USA) containing $160 \mathrm{mM}$ 1-Ethyl-3-(3-dimethylaminopropyl)carbodiimide (EDC, Acros Organics, USA) and $80 \mathrm{mM} \mathrm{N-Hydroxysuccinimide} \mathrm{(NHS,} \mathrm{Pierce,} \mathrm{USA)} \mathrm{and} \mathrm{incubated} \mathrm{in} 2$ $\mathrm{mL}$ of $4 \mu \mathrm{g} / \mathrm{mL}$ streptavidin solution in PBS at room temperature and $200 \mathrm{rpm}$ for $3 \mathrm{~h}$. The slides were removed from the streptavidin solution and the remaining solution was collected. Upon incubation, the streptavidin-functionalized glass slide was rinsed thoroughly with PBS to remove loosely bound proteins and the first $200 \mu \mathrm{L}$ of the washout was also collected, added to the supernatant, and used for protein loading analysis.

\section{Evaluation of the protein loading onto the functionalized glass slide}

The amount of the streptavidin bound to the glass slides was evaluated using micro BCA protein assay (Pierce, USA) and subtracting the amount of the protein washed out in the supernatant and collected washes from the amount of the protein initially added in the incubation step. Briefly, $500 \mu \mathrm{L}$ of working reagent containing 50 parts of reagent $\mathrm{A}, 48$ parts reagent $\mathrm{B}$ and 2 parts of reagent $\mathrm{C}$ (all reagents were provided with the kit) were mixed with $500 \mu \mathrm{L}$ streptavidin solution (either from the supernatant and or the $200 \mu \mathrm{L}$ of the washes) and incubated at $60{ }^{0} \mathrm{C}$ for $1 \mathrm{~h}$. After incubation and sample cooling to room temperature, absorbance at $562 \mathrm{~nm}$ was recorded using a UV-Vis Spectrophotometer (Thermo Scientific EVO300). Known concentrations of streptavidin in the working reagent were used to form a calibration curve.

\section{AFM force measurement}

A contact mode AFM force measurement was used to evaluate the rupture forces between biotin- or anti-streptavidin antibodies-coated tip and streptavidin-coated substrate. The trigger force was fixed to $3 \mathrm{nN}$ and the spring constant of the cantilever was measured and calibrated before each experiment using established calibration method ${ }^{34}$. The area for each analysis was kept constant to $1 \mu \mathrm{m} \times 1 \mu \mathrm{m}$.

\section{Statistical data analysis}

In order to compare the average rupture forces, a Student T-test was carried out with the significance level of $\mathrm{p}^{*}<0.05 ; 40$ parallel force map experiments per individual sample analysis (i.e., where sample is defined as either of the ligand) were performed, with at least 6 samples being analyzed at independent times. For the loading rate dependence of the rupture force there were at least 12 samples being analyzed. For each, there were at least 5 areas of 1 
$\mu \mathrm{m} \times 1 \mu \mathrm{m}$ being considered; in each one of these areas the loading force was varied. For the biotin-streptavidin, the loading rate varied from 250 to $150000 \mathrm{pN} / \mathrm{s}$, while for anti-streptavidin antibodies-streptavidin the loading rate varied from 11500 to $161000 \mathrm{pN} / \mathrm{s}$. These loading rates accommodated the different spring constant as measured for individual experiments. 


\section{Results and Discussion}

Applied biochemistry relies on molecular recognition as an essential element in evaluating self-replication, self-assembly, and information processing. Analysis of biomolecular recognition events performed in synthetic environment have shown that protein-ligand interactions are the key part of the bond formation, with the bond being strongly influenced by both steric hindrance ${ }^{35}$ and protein's arrangement at a particular interface ${ }^{36}$.

To evaluate and help rank biomolecular recognition events based on the bond energetics, we used the streptavidin-anti-streptavidin antibody complex. Streptavidin was chosen as a model protein based on previous reports that helped characterize both its structure ${ }^{37}$ as well as its interaction with known ligand biotin ${ }^{19,38,39}$.

\section{Experimental set-up to evaluate biomolecular recognition events between a protein and its ligands}

Our experimental approach used atomic force microscopy (AFM) in contact mode, streptavidin-coated surfaces and ligand-coated AFM tips and evaluated the rupture forces between proteins and their ligands at a constant trigger force. Specifically, Figure 1 shows the multi-step functionalization of glass surfaces with streptavidin. Briefly, by immersing glass slides into Piranha solution hydroxyl radicals were generated ${ }^{33}$ and subsequently used to immobilize 3-aminopropyltriethoxysilane (APTS) to be used as "hangers" for covalent binding of glutaraldehyde (GA; Figure 1a). Streptavidin was attached to the glutaraldehyde-functionalized surfaces using the zero length EDC/NHS chemistry ${ }^{40}$; complementary, anti-streptavidin antibody or biotin (used as control) ligands were immobilized onto the AFM tip by using physical binding ${ }^{39}$ (Figure 1b).

Figure 1c and Figure 1d depict the rupture force as result of engaging and de-engaging the ligand-functionalized tip from the streptavidin- coated surface under an external force controlled by the user (Figure 1c for the anti-streptavidin antibodies and 1d for biotin control). During the engaging process, the ligand-coated AFM tip came in direct contact with the streptavidin-coated surface to form ligand-protein conjugate complexes. During the tip detaching process and when the force generated by the bending of the cantilever reached the rupture force (with a more severe bent being a result of a higher rupture force between the coated tip and the streptavidin), the interaction between the ligand-coated tip and the streptavidin-coated surface was broken and recorded. 
a). Substrate

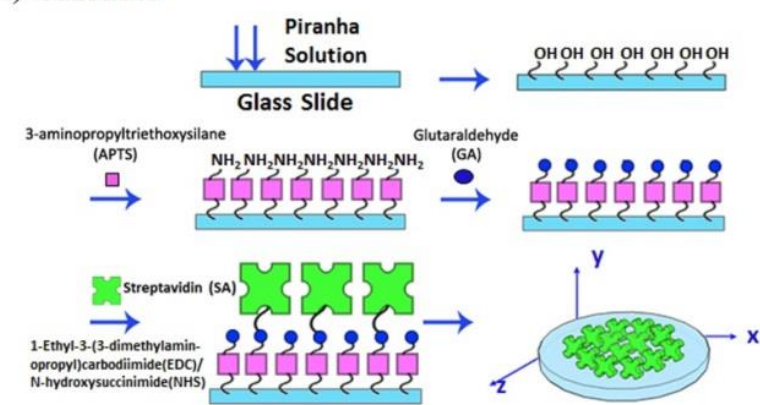

b) Tip

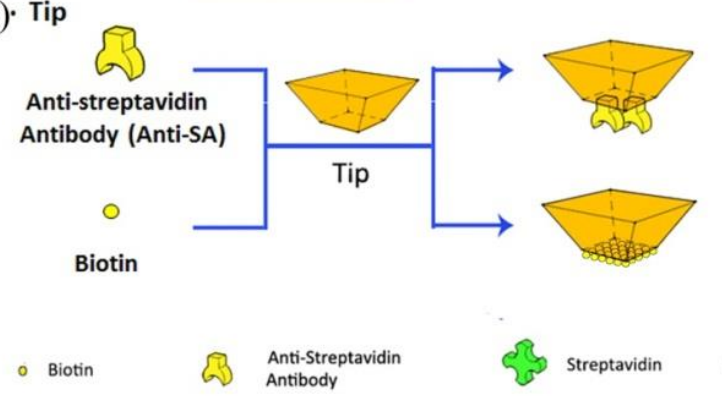

c)

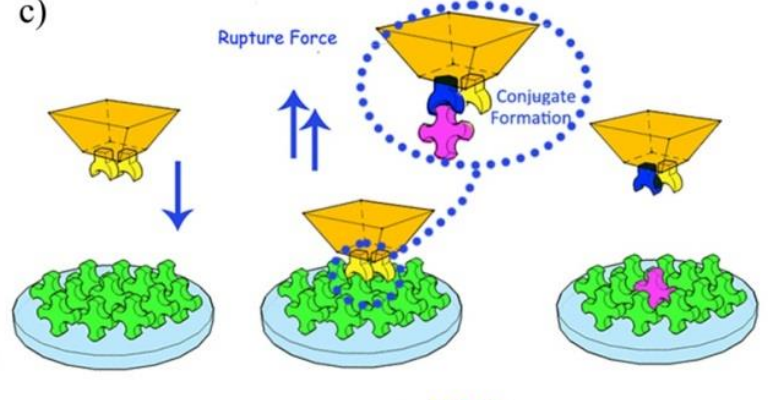

d)

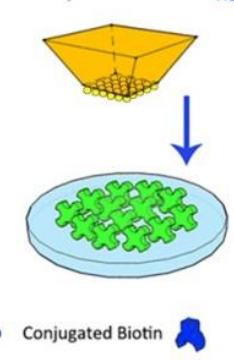

Figure 1: Concept drawing of surface functionalization and contact mode analysis performed using Atomic Force Microscopy (AFM). (a) Functionalization of streptavidin protein to the glass surfaces; functionalization was performed using covalent binding. (b) Functionalization of anti-streptavidin antibody or biotin to the AFM tip. Schematic representation of the rupture force between streptavidin and its ligands, i.e., anti-streptavidin antibodies (c), or biotin used as control (d) respectively.

To evaluate the rupture force between streptavidin and its ligands (i.e., biotin used as control and anti-streptavidin antibodies), two different cantilevers with spring constants of $20 \pm 6 \mathrm{pN} / \mathrm{nm}$ and $90 \pm 27 \mathrm{pN} / \mathrm{nm}$ respectively, were used. Since the effective spring constant depends on the elastic properties of both the cantilever and the substrate ${ }^{41}$, we used thermal tuning and analysis of the deflection versus distance curve at constant room temperature to calibrate the cantilevers before each experiment ${ }^{34}$. Thermal tuning analysis showed spring constants of $25 \pm 5 \mathrm{pN} / \mathrm{nm}$ and $104 \pm 14 \mathrm{pN} / \mathrm{nm}$ respectively; subsequently, the resulting rupture forces of the biotin-streptavidin (control) or anti-streptavidin antibodies-streptavidin complexes were evaluated at $1.2 \times 10^{5} \mathrm{pN} / \mathrm{s}$ and $5.4 \times 10^{5} \mathrm{pN} / \mathrm{s}$ force loading rates, with the loading rate being determined from the rate of cantilever retraction ${ }^{42}$. The rate of cantilever retraction was defined as the velocity at which the tip retracted from the streptavidin-coated surface resulting in retraction values between $4800 \pm 1000 \mathrm{~nm} / \mathrm{s}$ and $5192 \pm 616 \mathrm{~nm} / \mathrm{s}$ respectively for the two cantilevers being considered. 
Figures $2 \mathbf{a}$ and $\mathbf{2 b}$ shows the representative force maps of $1 \mu \mathrm{m} \times 1 \mu \mathrm{m}$ areas where the analysis of the rupture forces between the anti-streptavidin antibodies- or the biotin-coated tip and streptavidin-coated surfaces respectively were performed. For the contact mode AFM force analysis, the area underneath the tip was divided automatically into $10 \times 10$ matrices to result in 100 parallel test areas/per experiment, with the AFM measuring the rupture force upon contact between the ligand-coated tip and each one of these matrices and providing the average force map per individual area being analyzed. Each square was color-coded with the darker color representing a smaller rupture force while the lighter color referred to a larger value of such force.

\section{Analysis of protein-ligand rupture events}

Figures $2 \mathrm{c}$ and $2 \mathrm{~d}$ show the representative histograms force distribution associated with the rupture events, each derived from 100 independent matrices allowing for force measurements. For the anti-streptavidin antibody-streptavidin experiment, the rupture force ranged from 0 to $100 \mathrm{pN}$, with a dominant regime in the $25 \mathrm{pN}$ to $80 \mathrm{pN}$ range and two peaks (Figure 2c). The red solid line resulted from the multi-Gaussian fitting identified an average rupture force of about $42 \mathrm{pN}$ with $\mathrm{F}^{*} 1(68.7 \mathrm{pN})$ presumably indicating the most probable rupture force for a single bond formed between anti-streptavidin antibodies-coated tip and streptavidin-coated surface ${ }^{43,44}$. The force distribution also revealed another peak centered around $12.8 \mathrm{pN}$ presumably associated with the non-specific interaction between the tip and the surface or binding of the antibodies at other than the specific streptavidin pocket site. Indeed, in control experiments performed with anti-streptavidin antibodies-coated AFM tips brought in close proximity of the glass surface functionalized with only glutaraldehyde, showed a rupture force of only $12.2 \pm 6.8 \mathrm{pN}$.

For the control experiment performed between the biotin-coated tip and the streptavidin-coated surface (Figure 2d), the rupture force ranged from $5 \mathrm{pN}$ to $1340 \mathrm{pN}$, with a dominant regime in the 200 to $900 \mathrm{pN}$ range and with multiple peaks distribution ${ }^{45}$. Multi-Gaussian fit allowed identification of the average rupture force at around $551 \mathrm{pN}$ with peaks distribution at $\mathrm{F}^{*} 1(285 \mathrm{pN}), \mathrm{F}^{*} 2(636 \mathrm{pN})$ and $\mathrm{F}^{*} 3(973 \mathrm{pN})$ respectively presumably associated with rupture events for single, double and triple bonds ${ }^{46}$. Forces below $100 \mathrm{pN}$ were presumably due to non-specific interactions of the biotin-coated tip to the streptavidin-coated surface, non-specific interactions of the biotin-coated tip at other than the structural site of the attached streptavidin or the structural arrangement of the streptavidin at 
the glass interface that lead to a structural packing with limited biotin binding ability ${ }^{47}$, or an equilibrium between the association and dissociation process ${ }^{48,49}$ respectively. Indeed, control experiments showed that biotin-coated tips brought in close proximity of the glutaraldehyde-coated glass surfaces resulted in a rupture force of only about $8.6 \pm 7.1 \mathrm{pN}$.

a)

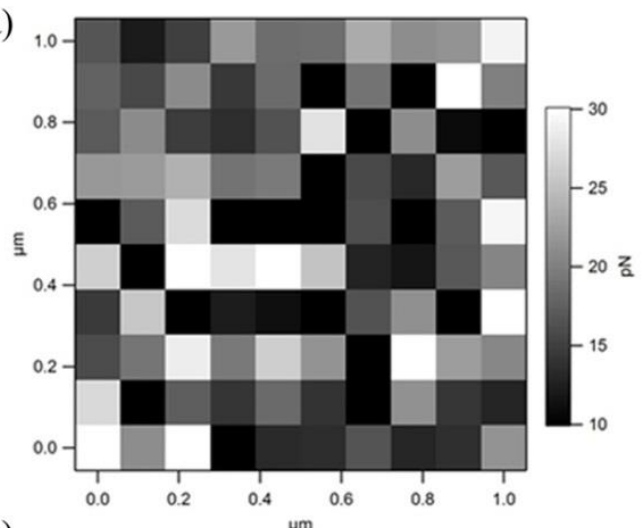

c)

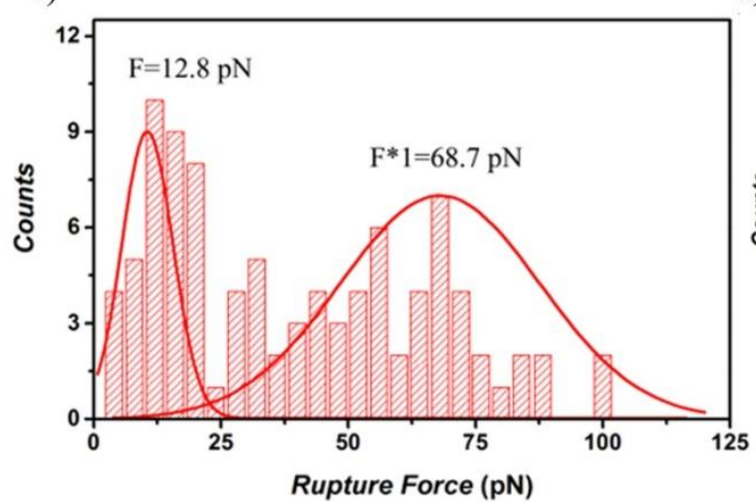

b)

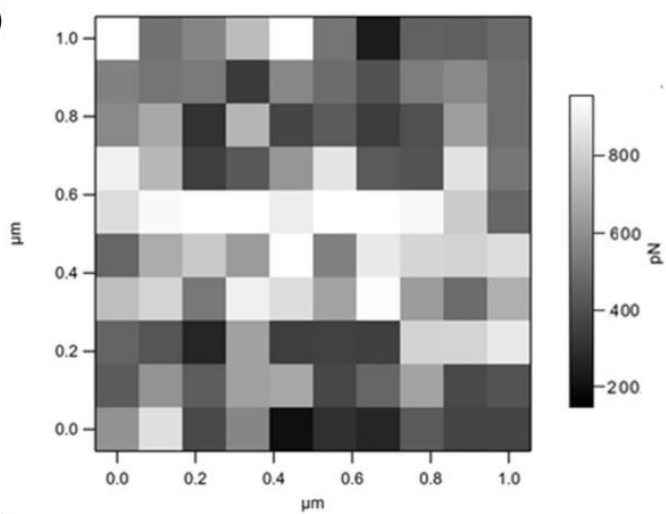

d)

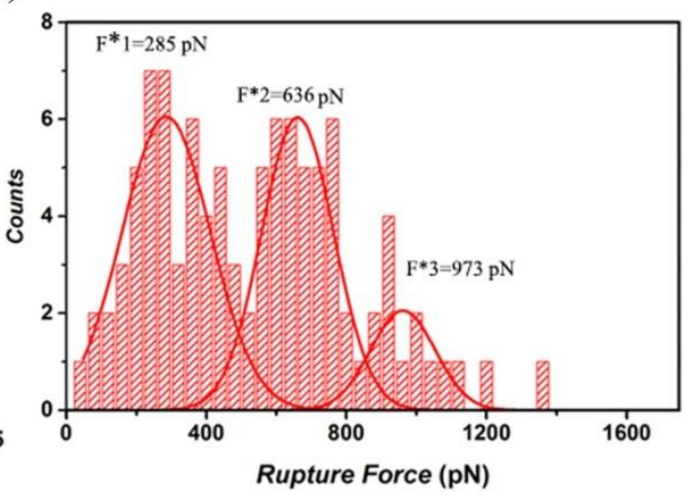

Figure 2: Binding forces between streptavidin and its ligands, namely anti-streptavidin antibodies and biotin used as control. (a) Contact mode force map analysis of the rupture force between representative anti-streptavidin antibody-coated tip and streptavidin-coated glass surface. (b) Contact mode force map analysis of the rupture force between representative biotin-coated tip and streptavidin-coated glass surface. The colored squares are associated with different values of the rupture forces being measured, with the darker representing smaller and brighter colors representing larger rupture forces respectively. (c) Representative distribution of the rupture forces between streptavidin-coated glass surface and anti-streptavidin antibody-coated tip. (d) Representative distribution of the rupture forces between streptavidin-coated glass surface and the biotin-coated tip. The distributions were obtained from independent force maps, with the two force maps for the individual ligands being analyzed being also representatives of all parallel experiments $(n=12)$. 
Figure 3 plots the mean rupture force against the loading rate for the unbinding process of the two different ligands ${ }^{50}$, with Figure 3a showing the loading rate dependence of the rupture force in the unbinding process of the anti-streptavidin antibody-coated tip and the streptavidin-coated surface and Figure 3b of the biotin-coated tip and the streptavidin-coated surface respectively. The analyses are based on at least 12 independent replicates performed for each of the protein-ligand complex being investigated. The red solid lines represent the linear fitting for the two protein-ligand complexes being considered, with each complex containing two regimes. Namely, for the anti-streptavidin antibody-streptavidin complex, the average rupture force between the coated tip and the streptavidin-coated surface showed a rapid decrease in the loading rate ranging from $690000 \mathrm{pN} / \mathrm{s}$ to $18400 \mathrm{pN} / \mathrm{s}$, followed by a more gradual decrease in the $161000 \mathrm{pN} / \mathrm{s}$ to $11500 \mathrm{pN} / \mathrm{s}$ range respectively. For the control with the biotin-coated tip, the rupture force between the functionalized tip and the streptavidin-coated substrate decreased with the loading rate. The average rupture force dropped from $601 \mathrm{pN}$ to $142 \mathrm{pN}$ as the loading rate dropped from $150000 \mathrm{pN} / \mathrm{s}$ to $7500 \mathrm{pNs}$. However, in the slower loading rate (ranging from $2500 \mathrm{pN} / \mathrm{s}$ to $250 \mathrm{pN} / \mathrm{s}$ ), the average rupture force was minimally changed (from $130 \mathrm{pN} / \mathrm{s}$ to $115 \mathrm{pN} / \mathrm{s}$ ) which could be due to the association and dissociation processes between the two ligands being at equilibrium ${ }^{48,49}$. Analysis also showed the presence of additional points in the loading range from 2980 to $8103 \mathrm{pN} / \mathrm{s}\left(\ln \left(\mathrm{L}_{\mathrm{r}}\right)=8 \sim 9\right)$ which were identified as being contained in a so-called "intermediate region".
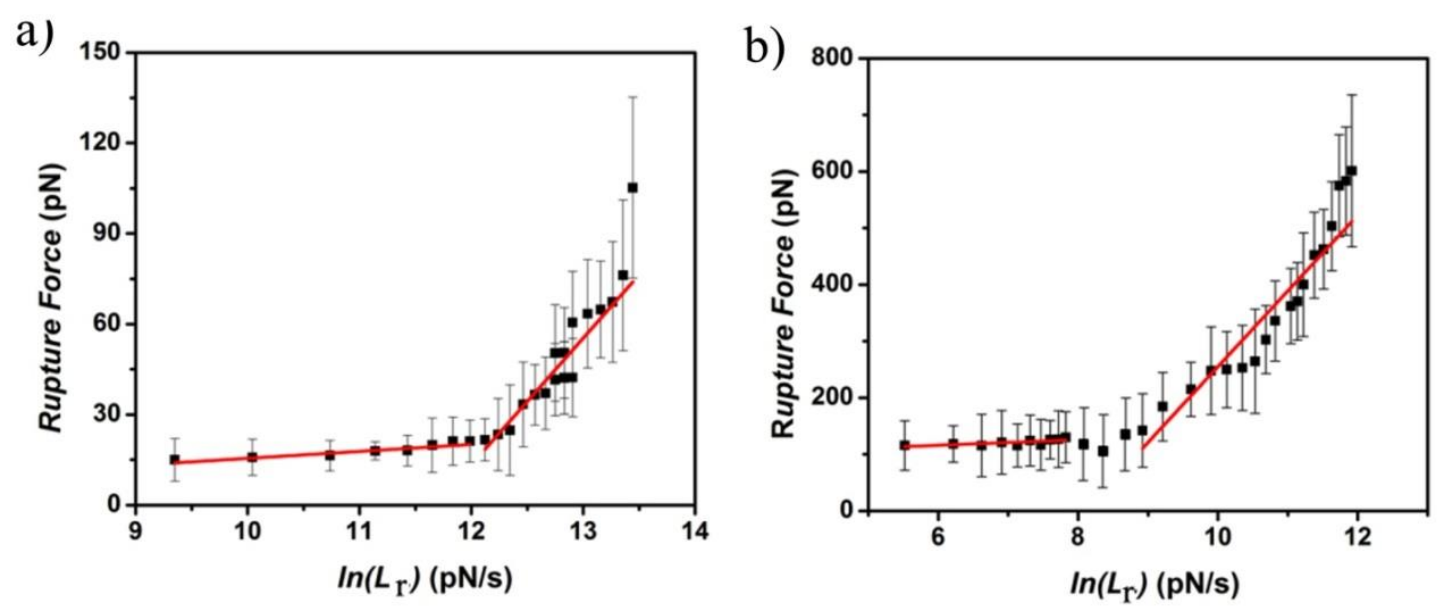

Figure 3: Loading rate dependence on the rupture force in the unbinding process of the (a) anti-streptavidin antibody-streptavidin; and (b) biotin-streptavidin respectively. The $\mathrm{x}$ axis is the logarithm of the loading rate while the y axis represents the rupture force; 12 independent force maps were obtained for each of the protein-ligand being investigated. 


\section{Kinetics of the association and dissociation of protein-ligand complexes recognition events}

For the quantitative analysis of the unbinding characteristics of the protein-ligand complexes, we employed Bell-Evans model ${ }^{51,52}$ and Kramer's theory ${ }^{53}$ and evaluated the dissociation rates and energy barriers. Briefly, Bell-Evans model predicts a linear relationship between the rupture force and the logarithm of the loading rates ${ }^{44}$, with the dissociation of the protein-ligand complex under an external force being correlated to the applied external force ${ }^{54}$ by:

$$
\mathrm{k}(\mathrm{F})=k_{0} \exp \left(\frac{F x^{\neq}}{k_{B} T}\right)
$$

where $\mathrm{k}(\mathrm{F})$ is the off-rate of protein-ligand dissociation that depends on the external force being applied $\left(\mathrm{F}_{\mathrm{x}}{ }^{\neq}\right), \mathrm{k}_{0}$ is the dissociation rate constant in the absence of an external force, $\mathrm{k}_{\mathrm{B}}$ is the Boltzmann constant, $\mathrm{T}$ is the absolute temperature, and $\mathrm{x}^{\neq}$is the energy barrier width which relates to the distance between the binding partners and the transition state projected along the direction of the applied force ${ }^{52}$. As the applied force increased with time and the deformation of the cantilever, the likelihood of any bond formed between the tip and the surface to be broken also increased, allowing for the probability density (P) of the "surviving" population of bonds to be calculated function of the failure of a single bond and the time required to break that bond, i.e., $t$, respectively using:

$$
P(\mathrm{t}, \mathrm{F})=\mathrm{k}(\mathrm{F}) \exp \left(-\int_{0}^{t} k\left(f\left(t^{\prime}\right)\right) d t\right)
$$

$P$ has a maximum value when $\mathrm{dP} / \mathrm{dF}=0$ and $\mathrm{F}=\mathrm{F}_{\max }$ respectively, where $\mathrm{F}_{\max }$ refers to the maximum force that could lead to the protein-ligand dissociation in one binding event and is function of the rupture force and loading rate through:

$$
\mathrm{k}\left(\mathrm{F}_{\max }\right)=\left.\mathrm{L}_{r} \frac{\partial}{\partial F} \ln (\mathrm{F})\right|_{\mathrm{F}=\mathrm{F}_{\max }}
$$

By combining equations 1 and 3 , and considering a constant loading rate $\mathrm{L}_{\mathrm{r}}$, the resulting 
unbinding force is:

$$
\mathrm{F}=\frac{k_{B} T}{x^{\neq}} \ln \left(\frac{L_{r} x^{\neq}}{k_{0} k_{B} T}\right) \quad \text { or } \quad \mathrm{F}=\frac{k_{B} T}{x^{\neq}} \ln \left(\frac{x^{\neq}}{k_{0} k_{B} T}\right)+\frac{k_{B} T}{x^{\neq}} \ln \left(\mathrm{L}_{\mathrm{r}}\right)
$$

From equation (4), the dissociation rate constant $\left(\mathrm{k}_{0}\right)$ for anti-streptavidin streptavidin and streptavidin-biotin (control) respectively were calculated and included in Table 1. Specifically, based on the linear fitting of the average rupture force versus the natural logarithm of the loading rates for anti-streptavidin antibodies-streptavidin in Figure 3a, the linear equations for the higher loading and lower loading rate regions respectively were: $F=41.98 \times \ln \left(\mathrm{L}_{\mathrm{r}}\right)-$ 490.4, and $\mathrm{F}=2.301 \times \ln \left(\mathrm{L}_{\mathrm{r}}\right)-7.513$. The resulting dissociation rate constants, $\mathrm{k}_{0}$ of the anti-streptavidin antibody-streptavidin complex were thus $2814 \mathrm{~s}^{-1}$ and $10.19 \mathrm{~s}^{-1}$, while the energy barrier widths, $\mathrm{x}^{\neq}$, were $0.097 \mathrm{~nm}$ and $1.73 \mathrm{~nm}$.

Analyses also showed that the values of the dissociation rate constants were smaller for the anti-streptavidin-streptavidin complex when compared to control biotin-streptavidin complexes thus suggesting that the bond lifetime $\left(1 / \mathrm{k}_{0}\right)$ of the anti-streptavidin-streptavidin complex is much smaller than that of the control. Specifically, the linear equations for the higher loading rate region (i.e., $\mathrm{F}=4.862 \times \ln \left(\mathrm{L}_{\mathrm{r}}\right)+86.78$ ), allowed determination of $\mathrm{k}_{0}$ as $24.94 \mathrm{~s}^{-1}$ and energy barrier width $\left(\mathrm{x}^{\neq}\right)$as $0.031 \mathrm{~nm}$ for the control. Complementary, for the lower loading rate region, the linear equation was $\mathrm{F}=133.87 \times \ln \left(\mathrm{L}_{\mathrm{r}}\right)-1084.01$ while the dissociation rate constant $\mathrm{k}_{0}$ was $3.64 \times 10^{-9} \mathrm{~s}^{-1}$ and $\mathrm{x}^{\neq}$was $0.84 \mathrm{~nm}$.

The above analyses confirmed previous studies that showed that there were at least two energy barriers (defined as inner and outer barriers respectively) for the biotin-streptavidin complex used as control, both presumably associated with the biotin binding close to the streptavidin pocket conformation (inner barrier) or at the last state before the unbound conformation (outer barrier) ${ }^{39,46}$. Similar results were obtained by Yuan et al., $(0.05 \mathrm{~nm})^{43}$ and Piramowicz et al., $(0.024 \pm 0.003 \mathrm{~nm})^{55}$ for the inner barrier, while for the outer barrier the results were 0.49 and $0.53 \mathrm{~nm}$ (Yuan et al., ${ }^{43}$ ) and $0.38 \pm 0.21 \mathrm{~nm}$ (Rico et al., ${ }^{39}$ ) respectively. The observed values were presumably related to the H-bond ruptures events as determined by the structural packing of the individual protein-ligand and the symmetry of the streptavidin tetramer itself ${ }^{46}$, with the outer energy barrier being attributed to the steric hindrance generated by the Trp120 amino acid which prevents the biotin from escaping during the unbinding process ${ }^{46}$. Yuan et al., ${ }^{43}$ also found that the outer barrier width decreases (from 
$0.49 \mathrm{~nm}$ to $0.31 \mathrm{~nm}$ ) while the $\mathrm{k}_{0}$ dissociation constant increases (from $1.67 \times 10^{-5} \mathrm{~s}^{-1}$ to $6.70 \times 10^{-3} \mathrm{~s}^{-1}$ ) after they used a truncated form of streptavidin with the Trp120 changed to Phe120.

Table 1: Bell Model Parameters from F vs $\ln \left(\mathrm{L}_{\mathrm{r}}\right)$ relationship

\begin{tabular}{ccccc}
\hline $\begin{array}{c}\text { Ligand-prote } \\
\text { in complex }\end{array}$ & $\begin{array}{c}\text { Loading } \\
\text { rate } \\
\text { (pN/s) }\end{array}$ & $\begin{array}{c}\text { Energy } \\
\text { barrier width } \\
\mathbf{x}^{\neq}(\mathbf{n m})\end{array}$ & $\begin{array}{c}\text { Dissociation rate } \\
\text { constant in the absence } \\
\text { of an external force }\end{array}$ & $\begin{array}{c}\text { Activation energy } \\
\mathbf{E}\left(\mathbf{k}_{\mathbf{B}} \mathbf{T}\right)\end{array}$ \\
$\begin{array}{c}\text { Anti-streptav } \\
\text { idin }\end{array}$ & $\begin{array}{c}11500 \text { to } \\
161000\end{array}$ & 1.73 & 10.19 & -2.32 \\
$\begin{array}{c}\text { antibody-stre } \\
\text { ptavidin }\end{array}$ & $\begin{array}{c}18400 \text { to } \\
690000\end{array}$ & 0.097 & 2814 & -7.94 \\
$\begin{array}{c}250 \text { to } \\
\text { Biotin-strept } \\
\text { avidin }\end{array}$ & 0.84 & $3.64 \times 10-9$ & 19.44 \\
& 7500 to & 0.031 & 24.94 & -3.2 \\
\hline
\end{tabular}

\section{Activation energy determination is function of the individual protein-ligand interactions}

Using Kramer's theory that describes the kinetic off rate for unbinding of the two partners in the individual complex and equation 4 , we calculated the activation energy of the protein-ligand interaction from the intercept and the slope in the individual linear fittings respectively, where the individual fittings were based on analysis of 12 independent replicates as:

$$
L_{r, F=0}=\omega f_{B} \exp \left(\frac{-\Delta E}{k_{B} T}\right)
$$

where $\omega$ is the frequency prefactor, $\mathrm{f}_{\mathrm{B}}$ is $\mathrm{k}_{\mathrm{B}} \mathrm{T} / \mathrm{x}^{\neq}$with $\Delta \mathrm{E}$ being the activation energy ${ }^{50}$.

$$
\frac{\Delta E}{k_{B} T}=-\ln \left(\mathrm{L}_{r, F=0}\right)+\ln \left(\mathrm{f}_{B} \omega\right) \approx-\ln \left(\mathrm{L}_{r, F=0}\right)+\ln \left(\mathrm{f}_{B}\right)
$$

By substituting $\mathrm{k}_{\mathrm{B}}$ and $\mathrm{x}^{\neq}$into equation (6), the activation energies of the biotin-streptavidin complex was calculated as $-3.20 \mathrm{k}_{\mathrm{B}} \mathrm{T}$ for the higher loading rate region and $19.44 \mathrm{k}_{\mathrm{B}} \mathrm{T}$ for the 
lower loading rate region respectively. Previous research showed that the activation energy for the biotin-streptavidin was in the range of $2.90 \mathrm{k}_{\mathrm{B}} \mathrm{T}$ to $35.91 \mathrm{k}_{\mathrm{B}} \mathrm{T}^{56,57}$.

The difference in between our and previously reported results is presumably related to the complexity of the experimental set-up in which both the loading rate range (that controlled the rupture events) and the surface functionalization method (that controlled attachment and packing of the ligand or the protein at their respective interfaces) were different. In particular, considering that each streptavidin when free in solution has 4 binding pockets for biotin, with the number of pockets decreasing upon protein immobilization, and considering that the very end of the tip is a square with the radius of $42 \mathrm{~nm}$ where the size of the biotin is about $3 \mathrm{~nm}$, the set-up geometry will allow for a large number of binding events to occur. Such events could lead to multi-bonds formation, all function of the overall distance of the coated cantilever from the substrate, the loading rate regime applied, the distribution of the binding pockets, the geometry of the protein and its ligands, as well as hindrance effects. These were confirmed by analyzing the "intermediate region" in which the outer barrier is governed by different interactions than the ones that govern the inner barrier also suggesting high enantioselectivities ${ }^{58}$ of the two binding partners, with the negative energies being presumably associated with conformational changes due to the binding of the ligand at other than the streptavidin pocket binding site itself $f^{59}$.

For the anti-streptavidin antibody-streptavidin complex, the activation energies were $-7.94 \mathrm{k}_{\mathrm{B}} \mathrm{T}$ for the higher and $-2.32 \mathrm{k}_{\mathrm{B}} \mathrm{T}$ for the lower loading rate regions respectively. Similar results were obtained for other antibody-antigen pairs with research showing an activation energy of $-6.37 \mathrm{k}_{\mathrm{B}} \mathrm{T}$ for the arginine-glycine-aspartate (RGD) and human platelet $\alpha_{\mathrm{IIb}} \beta_{3}$ pair ${ }^{60}$ or $-6.4 \mathrm{k}_{\mathrm{B}} \mathrm{T}$ for the RGD and fibrinogen pair $^{61}$. The negative values may illustrate that the binding event is not a two-state-one-step type, but it contains an intermediate step ${ }^{62}$ most likely associated with the re-arrangement of the two binding partners at the interfaces they were immobilized onto. The different values of the activation energy may also be due to the model being considered for the analysis ${ }^{63}$. It is arbitrarily however to speculate that there were two energy barriers in the anti-streptavidin antibody-streptavidin complex since the protein-ligand interaction that leads to this complex formation is more intricate than the one for the control biotin-streptavidin. In particular, the "Y"-shaped structure of the anti-streptavidin antibody with the two light and two heavy chains ${ }^{64}$ and its length of about $10 \mathrm{~nm}$ can assume at least 4 different orientations when coated onto the tip surface, i.e., "end-on fab-up", “end-on fab-down", "side-on" and "flat-on" 64 . Such orientations could 
presumably lead to reduced number of binding events with the protein-coated surface when in close proximity. While anti-streptavidin antibodies in the "end-on fab-up" structure have the highest probability to form a perfect bond with the immobilized streptavidin, other orientations resulting from either the protein or antibody's packing at their respective two interfaces, as well as steric hindrance, are likely to generate non-specific interactions and thus explain the lower energy barrier of the complex.

\section{Controlling the specificity of protein-ligand recognition events allows for extended in vitro applications}

The average rupture forces as resulted from all the parallel experiments performed for both anti-streptavidin antibody-streptavidin and biotin-streptavidin complexes are presented in Figure 4. As shown, the control biotin-streptavidin complex had an average rupture force of $551 \pm 132 \mathrm{pN}$ while for the streptavidin-anti-streptavidin antibody complex the rupture force was $50.2 \pm 12 \mathrm{pN}$. The rupture forces for other sets of controls, i.e., biotin or anti-streptavidin antibodies coated to the tip and brought in close proximity to only the glutaraldehyde-coated glass surfaces, were $8.6 \pm 7.1 \mathrm{pN}$ and $12.2 \pm 6.8 \mathrm{pN}$ respectively. Those additional controls were accounted to eliminate concerns related to ligand unbinding from the tip.

The performed Student's T-test showed that the average rupture forces between biotin-streptavidin and anti-streptavidin antibody-streptavidin complexes were significant from each other and significant from their controls respectively. The differences in the average rupture forces between the two pairs of ligands confirmed previous results that allowed calculations of the activation energy. In particular, with the activation energy of the biotin-streptavidin bond being much larger than that of the anti-streptavidin-streptavidin bond, a larger force will be required to cross that energy barrier. This analysis suggests that controlling the specificity of binding and limiting non-specific interactions through fine tuning of the protein-ligand interactions can help identify the disposition of protein binding pocket site, its packing at nanointerfaces, as well as shed light into its energetic characteristics as linked to a particular sequence/structure and the protein-ligand recognition event. 


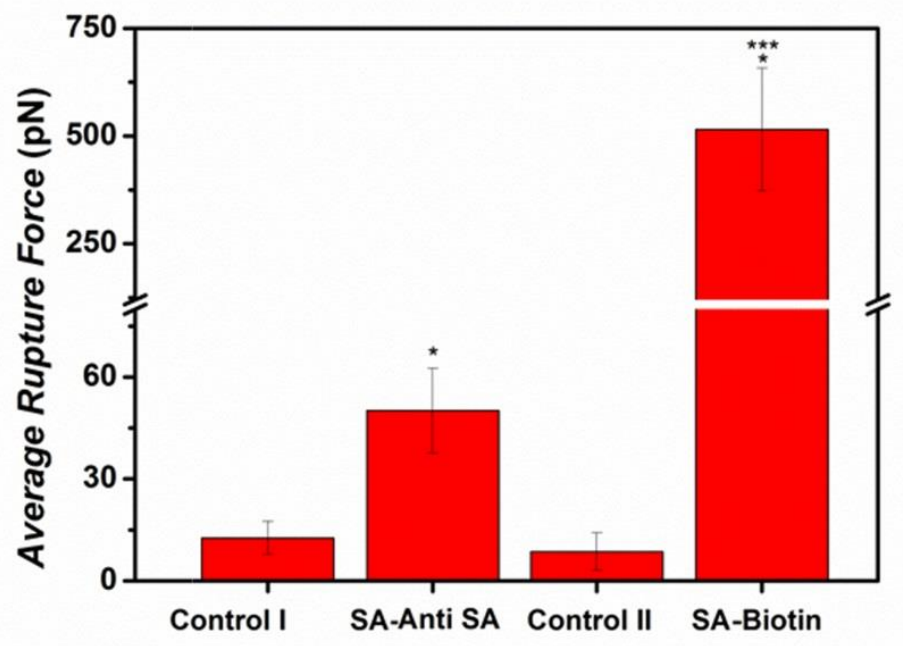

Figure 4.: Histogram of the average rupture force between streptavidin and its ligands, with at least 240 independent force maps analyses being performed for each of the complexes being investigated. Control I and control II represents the average rupture force between biotin- and anti-streptavidin antibody-coated tip and the glass respectively (with at least 60 independent force maps for each of the controls). A Student T-test was carried out with the significance level of $\mathrm{p}^{*}<0.05 ;\left(^{*}\right)$ indicates significance between individual complexes and the control while $(* * *)$ indicates significant from the different complexes.

Our study has the potential to advance biomedical applications. For instance, knowing that ELISA assay relies on the antibody immobilized on the substrate to capture the target that subsequently allows for a color change ${ }^{28}$, assays can be developed to recover the antibody-antigen and make the substrate reusable. Further, by applying a known rupture force to break a specific protein-ligand bond when using controlled microfluidic flow or differentiating between "perfect" versus "imperfect" bonds, immuno-separation assays can be optimized. Specifically, by considering the orientation of the antibodies at nanointerfaces, a higher sensitivity and selectivity could be achieved if all the antibodies will be in an "end-on fab-up" orientation to allow efficient capturing of the corresponding protein with the binding-breaking event performing as an "on-off switch" in vitro. Lastly, when the target binds to its receptor, the protein-ligand complex could be considered an "on" switcher while applying a specific force to break this bond will create an "off" switcher to be possibly used for diverse applications from biosensors to gene expression ${ }^{65}$. 


\section{Conclusions}

In this study, the energetics of anti-streptavidin antibody-streptavidin complex was studied by AFM and compared to that of the well-known biotin-streptavidin bond. Multi-Gaussian fitting showed that a multi-binding events occurred between the biotin-coated AFM tip and the streptavidin-coated glass surface, which are in contrast with a single binding event observed to occur between the anti-streptavidin antibody and the streptavidin surface. Results also showed that the "Y" shaped structure of the anti-streptavidin antibodies and their packing at the interfaces are more likely to form imperfect bonds with their protein ligand, thus decreasing their required rupture forces. Kinetic parameters, such as the energy barrier width, activation energy, dissociation constant of both protein-ligand complexes were obtained from Bell-Evan's model to help evaluate and categorize protein-ligand binding affinities possibly to be extended for biomedical applications. 


\section{References:}

1 Mogensen, T. H. Pathogen Recognition and Inflammatory Signaling in Innate Immune Defenses. Clinical Microbiology Reviews 22, 240-273, doi:10.1128/cmr.00046-08 (2009).

2 Akira, S., Uematsu, S. \& Takeuchi, O. Pathogen Recognition and Innate Immunity. Cell 124, 783-801 (2006).

3 Jamali, A. et al. A novel adjuvant, the general opioid antagonist naloxone, elicits a robust cellular immune response for a DNA vaccine. International immunology 21, 217-225, doi:10.1093/intimm/dxn139 (2009).

4 Abboud, N. et al. A requirement for FcgammaR in antibody-mediated bacterial toxin neutralization. The Journal of experimental medicine 207, 2395-2405, doi:10.1084/jem.20100995 (2010).

5 Pouyanfard, S., Bamdad, T., Parsania, M., Hashemi, H. \& Mohammadi, M. G. Impact of timing strategy of LIGHT, a new TNF superfamily on immune platform induced by HSV-1 gB DNA vaccine. Cytokine 50, 99-103, doi:10.1016/j.cyto.2009.12.012 (2010).

6 Peng, H.-P., Lee, K. H., Jian, J.-W. \& Yang, A.-S. Origins of specificity and affinity in antibody-protein interactions. Proceedings of the National Academy of Sciences 111, E2656-E2665, doi:10.1073/pnas.1401131111 (2014).

7 Santos, M. M., Marques, I., Carvalho, S., Moiteiro, C. \& Felix, V. Recognition of bio-relevant dicarboxylate anions by an azacalix[2]arene[2]triazine derivative decorated with urea moieties. Organic \& biomolecular chemistry (2015).

8 Zhang, W. et al. Identification of a juxtamembrane mechanosensitive domain in the platelet mechanosensor glycoprotein Ib-IX complex. Blood 125, 562-569 (2015).

9 Kilinc, D., Blasiak, A., O’Mahony, James J., Suter, Daniel M. \& Lee, Gil U. Magnetic Tweezers-Based Force Clamp Reveals Mechanically Distinct apCAM Domain Interactions. Biophysical journal 103, 1120-1129 (2012).

10 Zor, E., Bingol, H., Ramanaviciene, A., Ramanavicius, A. \& Ersoz, M. An electrochemical and computational study for discrimination of D- and L-cystine by reduced graphene oxide/beta-cyclodextrin. The Analyst 140, 313-321 (2015).

11 Sischka, A. et al. Dynamic translocation of ligand-complexed DNA through solid-state nanopores with optical tweezers. J Phys Condens Matter 22, 454121 (2010). 
12 Liebesny, P., Goyal, S., Dunlap, D., Family, F. \& Finzi, L. Determination of the number of proteins bound non-specifically to DNA. Journal of Physics: Condensed Matter 22, 414104-414108 (2010).

13 Duan, X. et al. Quantification of the affinities and kinetics of protein interactions using silicon nanowire biosensors. Nat Nano 7, 401-407 (2012).

14 Wang, C., Chowdhury, S., Gupta, S. K. \& Losert, W. Optical micromanipulation of active cells with minimal perturbations: direct and indirect pushing. Journal of biomedical optics 18, 045001 (2013).

15 Neuman, K. C. \& Nagy, A. Single-molecule force spectroscopy: optical tweezers, magnetic tweezers and atomic force microscopy. Nat Meth 5, 491-505 (2008).

16 Ulrichs, T., Drotleff, A. M. \& Ternes, W. Determination of heat-induced changes in the protein secondary structure of reconstituted livetins (water-soluble proteins from hen's egg yolk) by FTIR. Food chemistry 172, 909-920 (2015).

17 Müller, D. J. \& Dufrêne, Y. F. Atomic force microscopy: a nanoscopic window on the cell surface. Trends in Cell Biology 21, 461-469 (2011).

18 Scheuring, S. \& Dufrêne, Y. F. Atomic force microscopy: probing the spatial organization, interactions and elasticity of microbial cell envelopes at molecular resolution. Molecular Microbiology 75, 1327-1336 (2010).

19 Guo, S., Ray, C., Kirkpatrick, A., Lad, N. \& Akhremitchev, B. B. Effects of Multiple-Bond Ruptures on Kinetic Parameters Extracted from Force Spectroscopy Measurements: Revisiting Biotin-Streptavidin Interactions. Biophysical journal 95, 3964-3976 (2008).

20 Lesoil, C. et al. Molecular shape and binding force of Mycoplasma mobile's leg protein Gli349 revealed by an AFM study. Biochemical and Biophysical Research Communications 391, 1312-1317 (2010).

21 Ge, L., Jin, G. \& Fang, X. Investigation of the Interaction between a Bivalent Aptamer and Thrombin by AFM. Langmuir 28, 707-713 (2012).

22 Haynes, B. F. et al. Immune-Correlates Analysis of an HIV-1 Vaccine Efficacy Trial. New England Journal of Medicine 366, 1275-1286 (2012).

23 Ambrosi, A., Airò, F. \& Merkoçi, A. Enhanced Gold Nanoparticle Based ELISA for a Breast Cancer Biomarker. Analytical Chemistry 82, 1151-1156 (2009).

24 Wang, X. et al. QDs-DNA nanosensor for the detection of hepatitis B virus DNA and the single-base mutants. Biosensors and Bioelectronics 25, 1934-1940 (2010). 
25 Qian, P., Ai, S., Yin, H. \& Li, J. Evaluation of DNA damage and antioxidant capacity of sericin by a DNA electrochemical biosensor based on dendrimer-encapsulated Au-Pd/chitosan composite. Microchim Acta 168, 347-354 (2010).

26 Brennan, T. P. et al. Atomic Layer Deposition of CdS Quantum Dots for Solid-State Quantum Dot Sensitized Solar Cells. Advanced Energy Materials 1, 1169-1175 (2011).

27 Samanta, D. \& Sarkar, A. Immobilization of bio-macromolecules on self-assembled monolayers: methods and sensor applications. Chemical Society Reviews 40, 2567-2592 (2011).

28 Read, A. J. et al. Application of real-time PCR and ELISA assays for equine influenza virus to determine the duration of viral RNA shedding and onset of antibody response in naturally infected horses. Australian Veterinary Journal 89, 42-43 (2011).

29 Luime, J. J., Colin, E. M., Hazes, J. M. \& Lubberts, E. Does anti-mutated citrullinated vimentin have additional value as a serological marker in the diagnostic and prognostic investigation of patients with rheumatoid arthritis? A systematic review. Annals of the rheumatic diseases 69, 337-344, doi:10.1136/ard.2008.103283 (2010).

30 Wagner, C. A. et al. Identification of anticitrullinated protein antibody reactivities in a subset of anti-CCP-negative rheumatoid arthritis: association with cigarette smoking and HLA-DRB1 'shared epitope' alleles. Annals of the rheumatic diseases 74, 579-586, doi:10.1136/annrheumdis-2013-203915 (2015).

31 Szekanecz, Z. et al. Anti-citrullinated protein antibodies in rheumatoid arthritis: as good as it gets? Clinical reviews in allergy \& immunology 34, 26-31, doi:10.1007/s12016-007-8022-5 (2008).

32 Gan, S. D. \& Patel, K. R. Enzyme Immunoassay and Enzyme-Linked Immunosorbent Assay. J Invest Dermatol 133, e12, doi:10.1038/jid.2013.287 (2013).

33 Howarter, J. A. \& Youngblood, J. P. Optimization of Silica Silanization by 3-Aminopropyltriethoxysilane. Langmuir 22, 11142-11147 (2006).

34 Ohler, B. Cantilever spring constant calibration using laser Doppler vibrometry. Review of Scientific Instruments 78, - (2007).

35 Soteropulos, C. E., Hunt, H. K. \& Armani, A. M. Determination of binding kinetics using whispering gallery mode microcavities. Applied Physics Letters 99, - (2011).

36 Hinterdorfer, P. \& Dufrene, Y. F. Detection and localization of single molecular recognition events using atomic force microscopy. Nat Meth 3, 347-355 (2006). 
37 Freitag, S., Le Trong, I., Klumb, L., Stayton, P. S. \& Stenkamp, R. E. Structural studies of the streptavidin binding loop. Protein Science : A Publication of the Protein Society 6, 1157-1166 (1997).

38 González, M. et al. Interaction of Biotin with Streptavidin: THERMOSTABILITY AND CONFORMATIONAL CHANGES UPON BINDING. Journal of Biological Chemistry 272, 11288-11294 (1997).

39 Rico, F. \& Moy, V. T. Energy landscape roughness of the streptavidin-biotin interaction. Journal of Molecular Recognition 20, 495-501 (2007).

40 Campbell, A. S., Dong, C., Dordick, J. S. \& Dinu, C. Z. BioNano engineered hybrids for hypochlorous acid generation. Process Biochemistry 48, 1355-1360 (2013).

41 Cleveland, J. P., Manne, S., Bocek, D. \& Hansma, P. K. A nondestructive method for determining the spring constant of cantilevers for scanning force microscopy. Review of Scientific Instruments 64, 403-405 (1993).

42 Dudko, O. K., Hummer, G. \& Szabo, A. Intrinsic Rates and Activation Free Energies from Single-Molecule Pulling Experiments. Physical Review Letters 96, 108101 (2006).

43 Yuan, C., Chen, A., Kolb, P. \& Moy, V. T. Energy Landscape of Streptavidin-Biotin Complexes Measured by Atomic Force Microscopy†. Biochemistry 39, 10219-10223 (2000).

44 Morfill, J. et al. Affinity-Matured Recombinant Antibody Fragments Analyzed by Single-Molecule Force Spectroscopy. Biophysical journal 93, 3583-3590 (2007).

45 Wong, S. S., Joselevich, E., Woolley, A. T., Cheung, C. L. \& Lieber, C. M. Covalently functionalized nanotubes as nanometre- sized probes in chemistry and biology. Nature 394, 52-55 (1998).

46 Teulon, J. M. et al. Single and multiple bonds in (strept)avidin-biotin interactions. Journal of molecular recognition: JMR 24, 490-502 (2011).

47 Campbell, A. S. et al. Enzyme Catalytic Efficiency: A Function of Bio-Nano Interface Reactions. ACS Applied Materials \& Interfaces 6, 5393-5403, doi:10.1021/am500773g (2014).

48 Schönherr, H. et al. Individual Supramolecular Host-Guest Interactions Studied by Dynamic Single Molecule Force Spectroscopy. Journal of the American Chemical Society 122, 4963-4967, doi:10.1021/ja994040i (2000). 
49 Zou, S., Schönherr, H. \& Vancso, G. J. Force Spectroscopy of Quadruple H-Bonded Dimers by AFM: Dynamic Bond Rupture and Molecular Time-Temperature Superposition. Journal of the American Chemical Society 127, 11230-11231, doi:10.1021/ja0531475 (2005).

50 Lee, C.-K., Wang, Y.-M., Huang, L.-S. \& Lin, S. Atomic force microscopy: Determination of unbinding force, off rate and energy barrier for protein-ligand interaction. Micron 38, 446-461 (2007).

51 Bell, G. Models for the specific adhesion of cells to cells. Science 200, 618-627 (1978).

52 Evans, E. \& Ritchie, K. Dynamic strength of molecular adhesion bonds. Biophysical journal 72, 1541-1555 (1997).

53 Kramers, H. A. Brownian motion in a field of force and the diffusion model of chemical reactions. Physica 7, 284-304 (1940).

54 Bizzarri, A. R. \& Cannistraro, S. The application of atomic force spectroscopy to the study of biological complexes undergoing a biorecognition process. Chemical Society Reviews 39, 734-749 (2010).

55 de Odrowaz Piramowicz, M., Czuba, P., Targosz, M., Burda, K. \& Szymonski, M. Dynamic force measurements of avidin-biotin and streptavdin-biotin interactions using AFM. Acta biochimica Polonica 53, 93-100 (2006).

56 Galligan, E., Roberts, C. J., Davies, M. C., Tendler, S. J. B. \& Williams, P. M. Simulating the dynamic strength of molecular interactions. The Journal of Chemical Physics 114, 3208-3214 (2001).

57 Moy, V. T., Florin, E.-L. \& Gaub, H. E. Adhesive forces between ligand and receptor measured by AFM. Colloids and Surfaces A: Physicochemical and Engineering Aspects 93, 343-348 (1994).

58 Han, X. et al. Kinetic Evidence of an Apparent Negative Activation Enthalpy in an Organocatalytic Process. Sci. Rep. 3 (2013).

59 Mozurkewich, M., Lamb, J. J. \& Benson, S. W. Negative activation energies and curved Arrhenius plots. 2. Hydroxyl + carbon monoxide. The Journal of Physical Chemistry 88, 6435-6441 (1984).

60 Lee, I. \& Marchant, R. E. Force measurements on the molecular interactions between ligand (RGD) and human platelet $\alpha \mathrm{IIb} \beta 3$ receptor system. Surface Science 491, 433-443 (2001). 
61 Lee, I. \& Marchant, R. E. Molecular interaction studies of hemostasis: fibrinogen ligand-human platelet receptor interactions. Ultramicroscopy 97, 341-352 (2003).

62 Craig, M. E., Crothers, D. M. \& Doty, P. Relaxation kinetics of dimer formation by self complementary oligonucleotides. Journal of Molecular Biology 62, 383-401 (1971).

63 Guo, S., Lad, N., Ray, C. \& Akhremitchev, B. B. Association kinetics from single molecule force spectroscopy measurements. Biophysical journal 96, 3412-3422 (2009).

64 Wiseman, M. E. \& Frank, C. W. Antibody Adsorption and Orientation on Hydrophobic Surfaces. Langmuir 28, 1765-1774 (2012).

65 Kim, M. G. et al. Regulation of Toll-like Receptor-mediated Sestrin2 Induction by AP-1, Nrf2, and the Ubiquitin-Proteasome System in Macrophages. Toxicological sciences : an official journal of the Society of Toxicology (2015). 


\title{
Chapter 2: Bio-pen for direct writing of single molecules on user-functionalized surfaces
}

\begin{abstract}
Advancing ultrahigh resolution (below $10 \mathrm{~nm}$ ) direct writing technologies could lead to impacts in areas as diverse as disease detection, genetic analysis and nanomanufacturing. Current methods based on electron-beam, photo- or dip-pen nano-lithography are laborious and lack flexibility when aiming to generate single molecule patterns for application specific integration. We hypothesize that a novel strategy could be developed to allow for writing of parallel and yet individually addressable patterns of single molecules on user-controlled surfaces. The strategy is based on using the in vitro self-recognition of tubulin protein to assembly the rigid protofilaments of a microtubule, with such microtubule to be subsequently used as a "biopen" capable of writing "inks" of single kinesin molecules in user-defined environments. Our results show that single kinesin inks could be written under the chemical hydrolysis of adenosine triphosphate and observed under both atomic force and optical microscopy. Upon extending inks functionalities, integration of soft and hard materials for nanostructure assembly and complex single molecule pattern formation is envisioned.
\end{abstract}




\section{Introduction}

Single molecule or component direct writing could impact areas as diverse as nanomaterials and nanostructure assembly ${ }^{1,2}$, disease detection ${ }^{3}$ and genetic analysis ${ }^{4}$. Current methods for advancing nanomaterials/nanostructure formation are based on electron-beam lithography ${ }^{5}$, photolithography $^{6}$, polymer pen lithography ${ }^{7}$, or micro-contact printing $^{8}$ and are used to deposit or assembly dots ${ }^{9}$, lines ${ }^{10}$ and arrays ${ }^{11}$. Using electron-beam lithography for instance led to custom nanostructures' formation at a resolution of about 60 $\mathrm{nm}$ to be serving for grouping small numbers of nanometer-sized gold clusters and for the facile fabrication of nanosensors ${ }^{12}$. Hierarchical super-hydrophobic well-ordered secondary nanostructured surfaces were successfully produced by dual-scale electron-beam lithography for self-cleaning applications ${ }^{13}$. Complementary, in disease detection, UV nanoprinting led to the formation of large area arrays of goat anti-human kappa chains for fast, low-cost screening of leukemic cancer marker ${ }^{14}$. Similarly, rapid-disease screening assays for advancing genetic analysis immunoarrays ${ }^{15}$ were developed using microcontact printing; the same technique was also applied for the formation of DNA arrays ${ }^{16}$ for ultrasensitive and simultaneous detection of cancer biomarker prostate specific antigen (PSA) and interleukin-6 (IL-6) proteins in serum, all at sub-pg. $\mathrm{mL}^{-1}$ levels. However, such printing technologies do not work routinely in the sub-50-nm regime especially when features are to be made from mixed hard and soft materials ${ }^{15}$. Further, laborious and difficult steps are limiting techniques' flexibility when aiming to generate parallel and yet individual patterns of multicomponent nanostructures to increase integration and flexibility. Moreover, the light or heat sensitivity of the proteins and oligonucleotides used in genetic and disease testing platforms need integration of complex, additional and separate steps to be used for fabrication, however with many of such steps reducing both the activity and functionality of such biomolecules ${ }^{17}$.

To increase flexibility for manufacturing and implementation, dip-pen nanolithography $(\mathrm{DPN})^{18}$ and other DPN related technologies ${ }^{19,20}$ were proposed as viable alternatives. The high resolution, registration and direct-writing ability in both ambient and inert environments allowed the formation of specific structures such as $\operatorname{dots}^{21}, \operatorname{lines}^{22}$ and $\operatorname{circles}^{23}$ while eliminating the etching ${ }^{24}$ and avoiding cross-contamination ${ }^{25}$ normally associated with the other lithographic methods listed above. Further, the gentle writing ability led to the formation of patterns of biologically active proteins to be used for studying the hierarchical assembly processes of systems ${ }^{26,27}$ while DNA arrays ${ }^{28}$ were used for addressing bio-sensing-related sensitivity. However, even though increased versatility was achieved, 
DPN continues to rely on non-specific capillary force for transferring of an "ink" to a "paper" which could lead to propagation of contaminants ${ }^{29}$. Furthermore, the technique lacks the ability to transfer individual molecules in a one-by-one fashion onto nanometer-sized spaces and is only capable to achieve about $20 \mathrm{~nm}$ resolution ${ }^{27}$. The next generation of direct write technologies should not only allow for individual separation of single molecules, but also for parallel and yet individual sustainability and attainability of the molecule or written components' diversity, all while increasing randomness and complexity of the deposited materials and under ambient temperature and pressure conditions.

We propose to design the next generation of biological tools capable to create flexible single molecule patterns that have a high degree of individual, yet parallel characteristics and could be used for site-specific transformations and integration into protein-driven nanomanufacturing strategies. Our strategy is based on using a user-friendly approach in which a microtubule cytoskeletal filament serves as an affixed "biological pen" capable to be manipulated in vitro to provide the route for writing single molecules of kinesin 1 motor proteins, a.k.a. kinesin inks. In the cell, microtubules are giving structural integrity ${ }^{30}$ while serving as regular and uniform tracks for transport of vesicles ${ }^{31}$ or organelles ${ }^{32}$; microtubules are growing with their plus end (fast growing end) towards the cell periphery while the slow end (or minus end) is oriented towards the cell nucleus. Kinesin uses a microtubule track in vivo to progress to specific locations with processive $\mathrm{e}^{33}$ and coordinated $8 \mathrm{~nm} \mathrm{steps}{ }^{34}$, and in vitro with speeds below $1 \mu \mathrm{m} / \mathrm{s}$, all under the transformation of the chemical cycle of adenosine triphosphate (ATP) into mechanical work and towards the fast growing end (or plus end) of the microtubule ${ }^{35}$.

Our strategy demonstrates the ability to write individually addressable kinesin patterns, all with nanometer resolution as dictated by the kinesin molecular step, on user-engineered surfaces. Considering the genetic capability for kinesin functionalization, it is envisioned that different kinesin-tagged inks and thus complex nanostructure writing capabilities could be introduced in the future, all to lead to the formation of single molecule patterns with high feasibility and versatility for advancing areas as diverse as nanomanufacturing and disease detection. 


\section{Materials and Methods}

\section{Expression of fluorescently labeled kinesin molecular motor}

Plasmid pPF_dmKHC-EGFP encoding for the dmKHC-EGFP protein (molecular weight 91.7 $\mathrm{kDa}$ ), and consisting of the Drosophila melanogaster kinesin delta tail (dmKHC) linked to the C-terminal end of the EGFP (enhanced green fluorescent protein) his-tagged on the protein's C-terminus was obtained at West Virginia University. The coding sequence was copied by polymerase chain reaction (PCR) from the pPK124 plasmid, a kind gift of Prof. Jonathan Howard, Yale University. Briefly, a set of primers of known sequences (CAAAGGAGATATACATATGAGCGCAGAACGAGAAATTCC

CTCGCCCTTGCTCAC.GCTCCCACGCGGAACAAG respectively) were used; pPF_EGFP plasmid was a pTriEx-4 plasmid (Novagen, MA, USA) carrying the coding sequence for a C-terminal his-tagged EGFP protein.

The pPF_EGFP plasmid was first linearized by treatment with the NcoI endonuclease (New England Biolabs, USA). Both the linearized pPF_EGFP plasmid and the pPK124 PCR amplicon were then gel purified and assembled using a Gibson assembly kit (New England Biolabs, USA) and per the manufacturer's protocol. The assembled DNA sequences were subsequently introduced into E. Coli strain Stbl4 (Invitrogen, Fisher Scientific, USA); clones were screened by endonuclease treatment and small-scale protein expression followed by standard lab purification was applied.

The pPF_dmKHC-EGFP plasmid was transformed into the E. coli strain BL21(DE3) pLysS (Stratagene, Agilent Technologies, USA). Protein expression was induced with 1.0 $\mathrm{mM}$ Isopropyl $\beta$-D-1-thiogalactopyranoside (IPTG) at $16{ }^{\circ} \mathrm{C}$ for $18 \mathrm{~h}$. The cell pellet was subsequently resuspended into ice-cold lysis buffer containing $50 \mathrm{mM}$ tris(hydroxymethyl)aminomethane hydrochloride (Tris/ $\mathrm{HCl}$ ), $\mathrm{pH} 8.0,50 \mathrm{mM}$ sodium chloride $(\mathrm{NaCl}), 2 \mathrm{mM}$ magnesium chloride $\left(\mathrm{MgCl}_{2}\right), 0.1 \mathrm{mM}$ adenosine 5'-triphosphate (ATP), $2 \%$ Triton X-100, $10 \mathrm{mM} \beta$-mercaptoethanol, $0.4 \mathrm{mg} / \mathrm{ml}$ lysozyme, ethylenediaminetetraacetic acid (EDTA)-free protease inhibitor cocktail (reagents from Biotool.com, USA), $1 \mathrm{mM}$ phenylmethylsulfonyl fluoride (PMSF), $8 \%$ trehalose, and $10 \mathrm{U} / \mathrm{ml}$ of Pierce Universal Nuclease (Fisher Scientific, USA), in a volume of $10 \mathrm{~mL}$ of lysis buffer per $1 \mathrm{~g}$ of cell pellet. Any cellular debris was removed by centrifugation (performed at $20000 \mathrm{~g}, 4{ }^{\circ} \mathrm{C}$, for $10 \mathrm{~min}$ ), and the clear lysate was supplemented with $4 \mathrm{M} \mathrm{NaCl}$ to bring the final concentration to 0.5 M.

Expressed protein was purified using two Bio-scale mini IMAC cartridges (Bio-Rad 
Laboratories Inc., USA) in series installed in a BioLogic DuoFlow chromatography system (Bio-Rad Laboratories Inc., CA, USA) by running two buffers, i.e., the wash buffer (50 mM sodium phosphate $\left(\mathrm{Na}_{3} \mathrm{PO}_{4}\right), 0.3 \mathrm{M} \mathrm{NaCl}, 1 \mathrm{mM} \mathrm{MgCl}_{2}, 10 \mu \mathrm{M}$ ATP, $5 \mathrm{mM}$ $\beta$-mercaptoethanol, $10 \%$ glycerol, $\mathrm{pH} 8.0$ ) and the elution buffer (wash buffer with $0.5 \mathrm{M}$ imidazole) respectively. The protein was then eluted by imidazole gradient and concentrated into $1 \mathrm{~mL}$ volume. A second chromatographic step was applied for further purification; specifically, a Superdex 200 10/300 GL gel-filtration column (GE Healthcare Life Sciences, USA) equilibrated with storage buffer (100 mM imidazole, $300 \mathrm{mM} \mathrm{NaCl}, 1 \mathrm{mM} \mathrm{MgCl} 2,10$ $\mu \mathrm{M}$ ATP, $1.0 \mathrm{mM}$ 1,4-dithiothreitol (DTT), 10\% sucrose, $\mathrm{pH} 7.0$ ) was used. Lastly, the protein concentration was estimated using the Coomassie protein assay and bovine gamma globulin standard (reagents from Fisher Scientific, USA) while the purification process was monitored by classic sodium dodecyl sulfate polyacrylamide gel electrophoresis (SDS-PAGE).

\section{Synthesis of microtubule}

Microtubules were synthesized from free tubulin suspended in a microtubule polymerization solution according to established protocol $^{36}$. Briefly, the polymerization solution was obtained by vortexing $5 \mu \mathrm{L} 100 \mathrm{mM} \mathrm{MgCl}_{2}$, with $6 \mu \mathrm{L}$ dimethyl sulfoxide (DMSO, 99.7\%, Fisher Scientific, USA), $5 \mu \mathrm{L} 25 \mathrm{mM}$ guanosine-5'-triphosphate (GTP, Sigma, USA) and $9 \mu \mathrm{L}$ BRB80 buffer (formed from a mixture of $80 \mathrm{mM}$ piperazine-N,N'-bis(2-ethanesulfonic acid buffer, $1 \mathrm{mM} \mathrm{MgCl} 2$ and $1 \mathrm{mM}$ ethylene glycol tetraacetic acid (EGTA), pH 6.8; all reagents were purchased from Fisher Scientific, USA). To initiate microtubule polymerization, $2.5 \mu \mathrm{L}$ polymerization solution was mixed with $10 \mu \mathrm{L}$ of $4 \mathrm{mg} / \mathrm{mL}$ biotin and rhodamine labeled tubulin (Cytoskeleton Inc, USA) and the mixture was incubated at $37{ }^{\circ} \mathrm{C}$ for $30 \mathrm{~min}$. To stabilize the resulting microtubules, the solution was dispersed in $1 \mathrm{~mL}$ BRB80 buffer containing $10 \mu \mathrm{M}$ paclitaxel (Fisher Scientific, USA). The stabilized microtubules were kept at room temperature for experimental use.

\section{Inking of microtubules with kinesin molecules}

Kinesins' ability to bind to the lab-synthesized microtubules was evaluated using a non-hydrolyzable form of ATP. Briefly, $8 \mu \mathrm{L}$ of $10 \mu \mathrm{g} / \mathrm{mL}$ of kinesin expressed as previously described was mixed with $2 \mu \mathrm{L} 20 \mathrm{mM}$ adenylyl-imidodiphosphate (AMP-PNP, Sigma, USA) and incubated for $1 \mathrm{~h}$ at $4{ }^{\circ} \mathrm{C}$. The mixture was subsequently mixed with $20 \mu \mathrm{L}$ microtubules 
(prepared as previously described) and $10 \mu \mathrm{L}$ of BRB80 buffer containing $10 \mu \mathrm{M}$ paclitaxel; resulting solution was incubated for $30 \mathrm{~min}$ at room temperature. Upon incubation, the unbound kinesin was separated in the supernatant by using an Allegra 64R centrifuge (Beckman Coulter, USA) and 30,000 rpm spinning for $10 \mathrm{~min}$. The supernatant was evaluated using fluorescence microscopy (Nikon, USA). The resulting pellet was dissolved in $40 \mu \mathrm{L}$ BRB80 containing $10 \mu \mathrm{M}$ paclitaxel and used immediately.

\section{Functionalization of a glass substrate}

Glass substrates were coated with anti-kinesin antibodies using a covalent binding strategy $^{37}$. For this, glass slides $(\mathrm{d}=25 \mathrm{~mm}$, Corning, USA) were first ultrasonicated in DI water, 99\% ethanol (90\%, Fisher Scientific, USA), and again in DI water, with 30 min for each of the sonication windows. Secondly, the slides were dried under vacuum for 1 day then exposed to UV light for $30 \mathrm{~min}$. Thirdly, the glass slides were treated with Piranha solution (mixture of $96.4 \%$ sulfuric acid, $\mathrm{H}_{2} \mathrm{SO}_{4}$, and $30 \%$ hydrogen peroxide, $\mathrm{H}_{2} \mathrm{O}_{2}$, Fisher Scientific, USA) in a $3: 1$ volume ratio at $120{ }^{\circ} \mathrm{C}$ for $10 \mathrm{~min}$. Such cleaned glass slides were subsequently washed with DI water and dried under vacuum for an additional day. Upon drying, the slides were immersed in $1 \mathrm{ml}$ of 5\% 3-aminopropyltriethoxysilane (APTES; Fisher Scientific, USA) in toluene (99.5\%, Fisher Scientific, USA) and incubated at room temperature for $1 \mathrm{~h}$. Upon time elapse, the slides were washed thoroughly with DI water, toluene and DI water and subsequently immersed into $1 \mathrm{~mL} \mathrm{5 \%}$ glutaraldehyde (Acros Organics, USA) in $0.2 \mathrm{M} \mathrm{pH}$ 9.0 Tris-buffered saline (TBS, made from tris and hydrochloric acid, reagents purchased from Fisher Scientific, USA) at room temperature, for $1 \mathrm{~h}$, with shaking at $200 \mathrm{rpm}$. Lastly, the slides were extensively rinsed with TBS, activated for $15 \mathrm{~min}$ in a $1 \mathrm{~mL} 160 \mathrm{mM}$ 1-Ethyl-3-(3-dimethylaminopropyl)carbodiimide (EDC, Acros Organics, USA) and $80 \mathrm{mM}$ N-Hydroxysuccinimide (NHS, Pierce, USA) in 2-(N-morpholino)ethanesulfonic acid buffer (MES, pH 4.7, Fisher Scientific, USA) and lastly incubated in $1 \mathrm{~mL}$ of $1 \mathrm{mg} / \mathrm{mL}$ anti-kinesin antibody (Antibodies Online, USA) in BRB80 at room temperature and $200 \mathrm{rpm}$ for $3 \mathrm{~h}$ respectively. Upon incubation, the functionalized glass slides were rinsed thoroughly with BRB80 to remove loosely bound antibodies.

\section{Functionalization of an Atomic Force Microscope tip}

A TR-400PB tip (Asylum Research, USA) was functionalized with kinesin-inked microtubule. For this, the tip was first cleaned by immersion in $1 \mathrm{~mL}$ of deionized (DI) water 
for $10 \mathrm{~min}$, secondly by immersion in acetone (99.5\%, Fisher Scientific, USA) for another 10 min, and thirdly by immersion in DI water for an additional $10 \mathrm{~min}$. Subsequently, the tip was dried under air, exposed to ultraviolet (UV) light for $30 \mathrm{~min}$, rinsed with $100 \mathrm{mM} \mathrm{pH} 7.0$ phosphate buffered saline (PBS, made from mono-potassium phosphate $\left(\mathrm{KH}_{2} \mathrm{PO}_{4}\right)$, dipotassium hydrogen phosphate $\left(\mathrm{K}_{2} \mathrm{HPO}_{4}\right)$ and $\mathrm{NaCl}$, all reagents purchased from Fisher Scientific, USA) and incubated in a solution of $50 \mathrm{mg} / \mathrm{mL}$ anti-tubulin antibody (Sigma, USA) for $3 \mathrm{~h}$ at $4{ }^{\circ} \mathrm{C}$. Upon incubation, the tip was rinsed with BRB80 to remove loosely bound antibodies. Lastly, $40 \mu \mathrm{L}$ kinesins-inked microtubule solution was dropped onto the tip surface and incubated for $10 \mathrm{~min}$ at room temperature; upon incubation, the tip was rinsed with BRB80 buffer containing $10 \mu \mathrm{M}$ paclitaxel (Supporting Information Scheme S1). The functionalized tip was used immediately upon preparation (see below).

\section{Kinesin writing onto a functionalized glass substrate}

The inked tip was loaded onto the AFM head of a MFP-3D Bio (Asylum Research, USA) and engaged in close proximity of the anti-kinesin antibody functionalized substrate in 100 $\mu \mathrm{L}$ gliding solution (BRB80 buffer containing 1mM MgATP, $20 \mathrm{mM}$ D-glucose; both reagents were from Fisher Scientific, USA), $0.02 \mathrm{mg} / \mathrm{mL}$ glucose oxidase, $0.8 \mathrm{mg} / \mathrm{mL}$ catalase (both reagents from Sigma, USA) and 0.5\% $\beta$-mercaptoethanol (Fisher Scientific, USA). Specifically, the set point, deflection and trigger point were set at $-0.5 \mathrm{~V},-2.5 \mathrm{~V}$ and 3 $\mathrm{nN}$ respectively. The AFM head/tip was lowered manually until the $\mathrm{Z}$ voltage decreased to about $70 \mathrm{~V}$. Subsequently the tip was engaged in the gliding solution for $1 \mathrm{~min}$ and $5 \mathrm{~min}$ to realize the writing process in contact mode (Supporting Information Scheme S1).

Kinesin writing onto anti-kinesin functionalized glass slides was evaluated using an ATP-based assay ${ }^{38}$ and observed using both fluorescence and atomic force microscopy. For this, $100 \mu \mathrm{L}$ gliding solution (BRB80 buffer containing 1mM MgATP, 20 mM D-glucose; both reagents were from Fisher Scientific, USA), $0.02 \mathrm{mg} / \mathrm{mL}$ glucose oxidase, $0.8 \mathrm{mg} / \mathrm{mL}$ catalase (both reagents from Sigma, USA) and 0.5\% $\beta$-mercaptoethanol (Fisher Scientific, USA) were added onto the tip functionalized with the kinesin-inked microtubule and incubated for $5 \mathrm{~min}$ at room temperature. The writing process was observed using a fluorescent microscope and a $100 \mathrm{x}$ objective $(\mathrm{NA}=1.4)$ under the DAPI and GFP filters and under an exposure time of $8.3 \mathrm{~s}$. The obtained patterns of kinesin were evaluated using AFM in contact mode as described below. 


\section{Nanoscopic morphological analyses}

Morphology analyses of microtubules, kinesin, antibody-functionalized surfaces or surfaces contained the printed kinesins were performed using the MFP-3D Bio in contact mode. For these, the cantilever's spring constant was calibrated using the thermal noise method $^{39}$ and the scan rate of the tip was fixed at $0.5 \mathrm{~Hz}$. At least 6 experiments for each one of samples have been performed.

For microtubule, mica sheets washed with DI water, ethanol and again with DI water, and subsequently dried overnight under vacuum and at room temperature, were used as substrates. The mica was functionalized with $20 \mu \mathrm{L}$ APTES through incubation at room temperature for $15 \mathrm{~min}$. Upon incubation, the mica was washed with toluene and DI water; any remaining solution was removed under vacuum. For the physical characteristics of the microtubules, $20 \mu \mathrm{L}$ of the sample with $0.5 \%$ glutaraldehyde was dropped onto the APTES functionalized mica and incubated for $1 \mathrm{~h}$ at room temperature. After the incubation, the surface was washed with $40 \mu \mathrm{L}$ BRB80 containing $10 \mu \mathrm{M}$ paclitaxel for at least 3 times and subsequently imaged.

For the evaluation of kinesin and surfaces contained printed kinesins, $20 \mu \mathrm{L}$ of the respective sample was dropped onto APTES functionalized glass slide (see above) and incubated for $1 \mathrm{~h}$ at room temperature. After the incubation, the surface was washed with 40 $\mu \mathrm{L}$ BRB80 buffer containing $10 \mu \mathrm{M}$ paclitaxel for at least 3 times and subsequently imaged.

Contact mode AFM was performed to evaluate the functionalized surfaces' roughness. The obtained images were flattened to remove curvature and slope effects from images. The root mean square average of height deviation (as taken from the mean image data plane) and the arithmetic average of absolute values of the surface height deviations (i.e., $R_{q}$ and $R_{a}$ ) were calculated from the obtained flattened images. For the clean glass surface, APTES functionalized glass slide surface, APTES-glutaraldehyde-anti-kinesin antibodies functionalized surface and APTES-glutaraldehyde-anti-kinesin antibodies-kinesin functionalized surfaces, the evaluated areas were $5 \mu \mathrm{m} \times 5 \mu \mathrm{m}$. The roughness of APTES-glutaraldehyde-anti-kinesin antibodies-kinesin functionalized surface was also evaluated on $1 \mu \mathrm{m} \times 1 \mu \mathrm{m}$ areas. Lastly, for evaluation of the printed kinesin, the AFM tip was removed from the buffer after the printing process ( $1 \mathrm{~min}$ and $5 \mathrm{~min}$ respectively) and the surface substrate was washed thoroughly with BRB80 buffer containing $10 \mu \mathrm{M}$ paclitaxel and subsequently imaged under AFM as described above. 


\section{Results and Discussion}

Nanoscale printing or direct writing has revolutionized applications ranging from analysis of biochemical mixtures ${ }^{40}$, to medicine ${ }^{41}$ and molecular-scale electronics ${ }^{42}$. However, in such applications, there are challenges associated with the high-throughput nature of the printed patterns or arrays of patterns, as well as with the limited parallelization flexibility that could allow for single molecule placement under ultrahigh resolution capability (below $10 \mathrm{~nm})^{43}$. We hypothesize that a novel strategy could be developed to allow for direct writing of parallel and yet individual addressable patterns of single molecules on user-controlled surfaces. The strategy is based on using rigid filaments of cellular microtubules of $\mathrm{nm}$ diameters as pens to write inks of single kinesin molecules, in user-defined environments.

To define the characteristics of the hypothesized writing strategy, we first demonstrated the feasibility of creating the microtubule pen (i.e., biopen). Specifically, we used rhodamine labeled tubulin as precursor for polymerizing microtubule in synthetic environment under the chemical energy of guanosine triphosphate $(\mathrm{GTP})^{36}$; the overall biopen length was evaluated using optical microscopy (Supporting Information). The resulting pen was "inked" with lab-expressed green fluorescent kinesin (Figure 1a). Kinesin's ability to ink the microtubule pen was evaluated under the non-hydrolazable form of adenosine-5-triphosphate (ATP), i.e. adenylyl-imidodiphosphate (AMPPNP), known to be acting as a kinesin-microtubule binding stabilizer $^{44}$. Previous studies have showed that kinesin-AMPPNP complex recognizes and binds to the microtubule stably, with a submicromolar $\mathrm{k}_{\mathrm{d}}$ and a $\mathrm{k}_{\text {off }}$ of $\sim 0.0025 \mathrm{~s}^{-1} 45$.

Contact mode atomic force microscopy (AFM) was used to confirm kinesin's ability to recognize the microtubule pen and evaluate an overall inking efficiency. For this, the microtubule inked with kinesin-AMPPNP complexes was first cross-linked with glutaraldehyde through Schiff base and Michael-type reactions ${ }^{46}$, immobilized onto 3-aminopropyltriethoxysilane (APTES) self-assembled monolayer (SAM) derived surfaces ${ }^{47}$ and subsequently scanned (Figure $\mathbf{1 b}$ and $\mathbf{c}$ respectively). 
a)

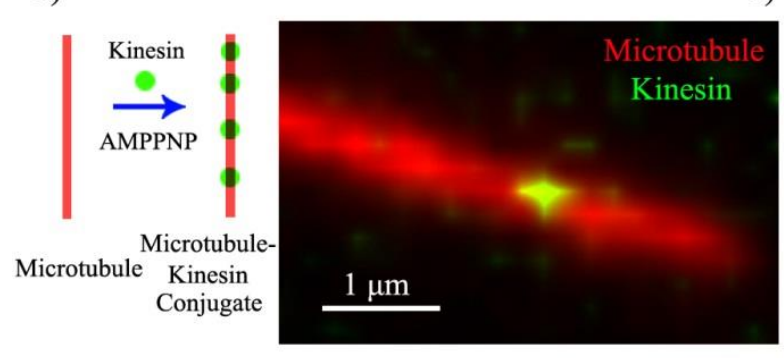

c)

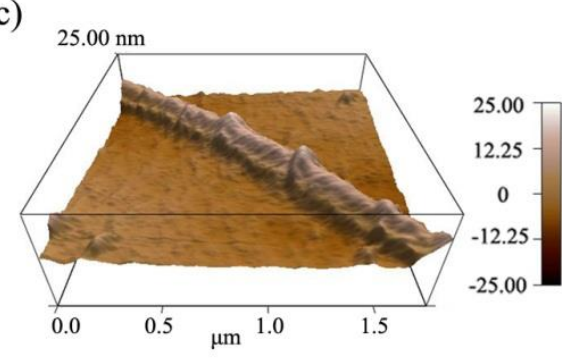

e)

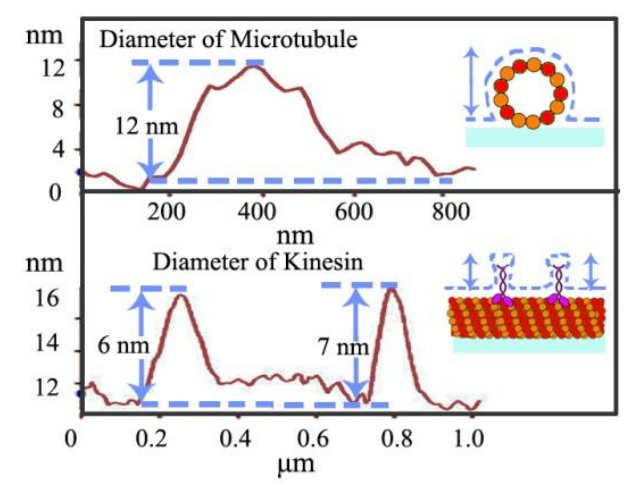

d)

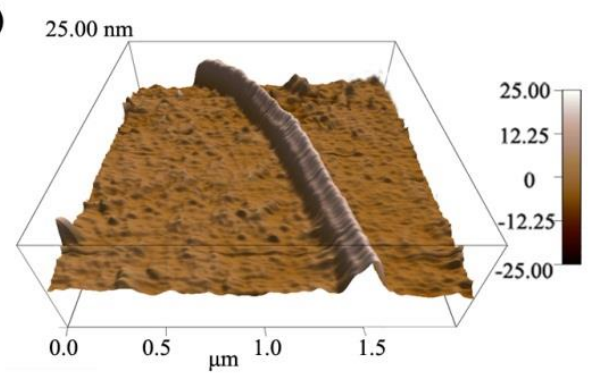

f)

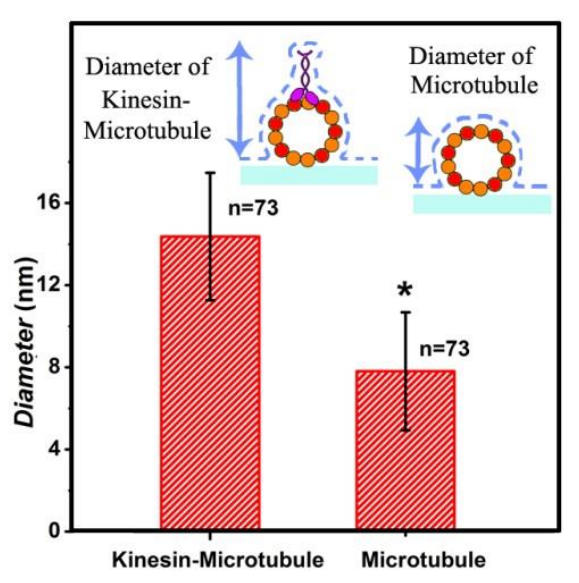

Figure 1: (a) The structure of the proposed kinesin-inked microtubule pen and its in vitro optical representation; microtubule is shown in red and kinesin is shown in green. (b) Schematic of the contact mode AFM analysis of the kinesin-inked microtubule pen. (c) Representative morphology of the kinesin-inked microtubule pen. The beads represent individual kinesin molecules bound to the surface of an immobilized microtubule, under the chemical energy of adenylyl-imidodiphosphate (AMPPNP). (d) Representative morphology of a microtubule used as control. (e) Height profile analysis help evaluate the distributions of kinesin inks onto a microtubule pen. Upper image: readings are done across the sample to evaluate the changes in height as associated with individual bound kinesins. Lower image: readings follow sample's profile and help identify the change in the microtubule local diameter as a change in the height profile when a bound kinesin is encountered. (f) Average diameters of the kinesin-inked microtubule pens and of control microtubules respectively. Student's T-test considered significance level of * $\mathrm{p}<0.05$. 
Representative morphology of the kinesin-inked microtubule biopen is shown in Figure 1d. Single kinesin molecules are seen as beads-like geometries distributed onto the linear structure of a surface immobilized microtubule. Control microtubule revealed a smooth, linear surface (Figure 1d). Analysis showed that the bead distribution led to a local increase in the microtubule's horizontal diameter (Figure 1e and 1f) from an average of $7.8 \mathrm{~nm}$ to an average of $14.4 \mathrm{~nm}$ (73 cases analyzed; herein the horizontal diameter is a reflection of the changes in height ${ }^{48}$ as recorded by the contact mode $\mathrm{AFM}^{49}$ ). Within such increase, individual kinesins diameters varied between 2 to $8 \mathrm{~nm}$. The observed variation in kinesin's diameter was presumably due to the different orientations that a kinesin bound molecule could assume at the microtubule's interface ${ }^{50}$, i.e., a straight ${ }^{51}$ or a stretched along the length of one or more of the microtubule protofilaments conformation ${ }^{50,52}$ respectively. Our analysis are supported by previous reports that show kinesin dimers immobilized onto surface immobilized microtubules to lead to average $5 \mathrm{~nm}$ changes in microtubule's diameter ${ }^{53}$. Also, Kacher et al., ${ }^{54}$ showed that single kinesin heads lead to $6 \times 3.5 \times 3 \mathrm{~nm}^{3}$ changes in microtubule diameter while $\mathrm{Hu}$ et al., ${ }^{55}$ showed sizes of $2 \sim 7 \mathrm{~nm}$ as associated with single kinesin molecules immobilized at such interface.

Upon demonstrating kinesin's ability to recognize and ink a microtubule pen, we created a "holder" capable to allow affixing of the microtubule biopen. The holder consisted of a biologically inert AFM tip functionalized with anti-tubulin antibodies (Figure 2a) where the efficacy/reproducibility of the loading of the biologically inert tip was controlled by the local distribution of the anti-tubulin antibodies. Figure $\mathbf{2 b}$ shows the kinesin-inked pen affixed onto the AFM tip through site specific interactions based on ligand recognition reactions between tubulin (from the pen) and anti-tubulin antibodies (immobilized at the tip) as observed under optical microscopy. Nonspecific kinesin attachment (green tip co-localization) was noted being presumably due to kinesin's strong submicromolar binding affinity ${ }^{56}$. 

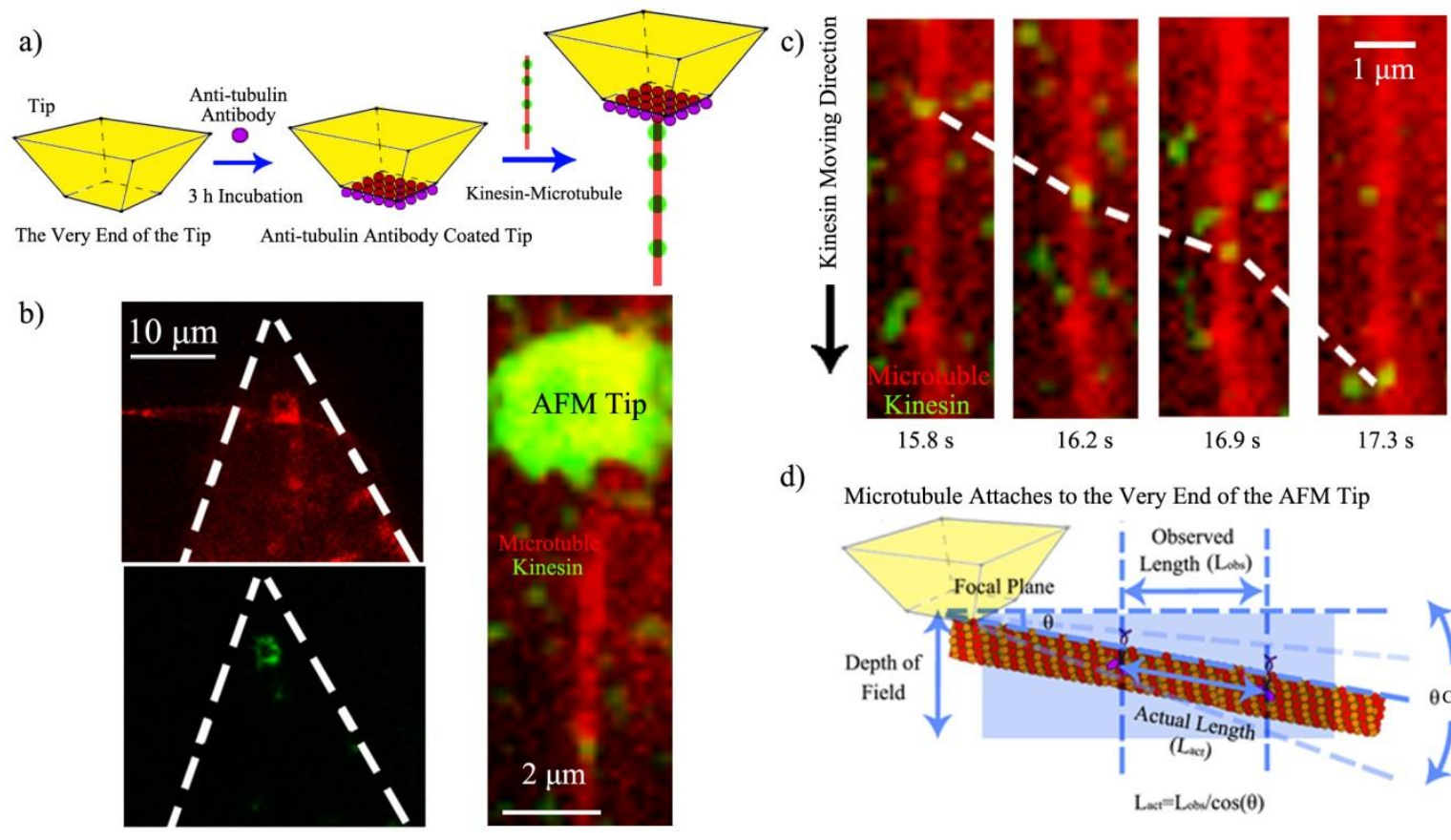

d)

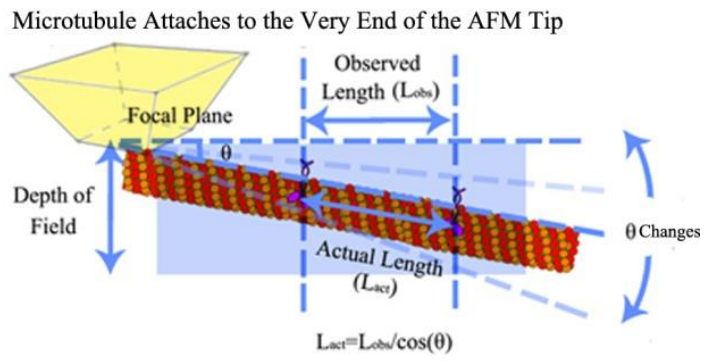

Figure 2: a) Schematic drawing of the AFM tip functionalization with anti-tubulin antibodies for controlled affixing of the kinesin-inked pen. b) Left upper: optical microscopy image showing the microtubule pen immobilized onto the tip through tubulin-anti-tubulin specific interactions. Left lower: optical microscopy image showing green kinesin inks onto the immobilized microtubule. The white dashed lines depict tip's profile. Right: representative fluorescence image of the kinesin-microtubule pen affixed at the tip formed from the merged images of red (microtubule) and green (labeled kinesin) signals. c) Representative fluorescent images of the kinesin ink (green) moving onto an affixed microtubule pen (red). The distance traveled allows for a measurable speed of about $3 \mu \mathrm{m} / \mathrm{s}$. d) The relationship between the visually observed length and the actual length that kinesin could travel onto an affixed pen. The different orientations of the affixed microtubule led to the different angles $(\theta)$ of observation. 
Upon demonstrating the successful affixing of the kinesin-inked microtubule pen at the AFM tip, we evaluated the ability of the pen to "dispense" such ink. Herein the dispensing is defined as the ability of kinesin to move along the affixed microtubule, under the exchangeable ATP energy; it is known that in kinesin moving, a single $8 \mathrm{~nm}$ step is correlated to an individual ATP hydrolysis event ${ }^{34}$.

Optical microscopy analysis confirmed the ability of the pen to dispense the inked kinesin (Figure 2c; also Supporting Information Figure S1). The average dispensing speed (defined as the total length that the kinesin is able to travel onto the affixed microtubule over a period of time) was $1.67 \pm 1.42 \mu \mathrm{m} / \mathrm{s}$, as calculated from 29 independent experiments. The large variation in the dispensing speed was presumably due to the different orientations that an affixed microtubule could have at the tip (Figure 2d) and/or the limited resolution available for the single molecule optical signal processing. Specifically, an attached microtubule could have different orientation angles $(\theta)$ relative to the focal plane of the tip to lead to a variation in the observed length traveled $\left(\mathrm{L}_{\mathrm{obs}}\right)$ versus the actual length $\left(\mathrm{L}_{\mathrm{act}}\right)^{57}$ being traveled; further, standard microscopy ability to capture height distributions of specific fluorophore-labeled structures was shown to be limited by the signal co-localization ${ }^{58}$.

Upon evaluating the ability of the affixed pen to dispense its ink, we developed a strategy for "capturing" such ink at specific location on a user-functionalized surface (Supporting Information Scheme S1). For this, it was proposed that the affixed kinesin-inked microtubule pen (with kinesin bound in the AMPPNP state) needed to be brought in close proximity to an anti-kinesin antibodies coated surface. In an initial first step, anti-kinesin antibodies used to allow affixing of the pen were immobilized through a multi-step strategy by using APTES as precursors. Aldehyde groups of glutaraldehyde were also used for zero-length chemistry to allow formation of imine linkages with the primary amine groups onto the kinesin molecules (Figure 3a) ${ }^{59}$. Anti-kinesin antibody surface functionalization was demonstrated using surface roughness analysis performed under contact mode AFM (Figure 3b, Supporting Information Eq. 1 and 2), on $4 \mu \mathrm{m}^{2}$ areas. Specifically, analysis of the surface height deviation (i.e., $\left.\mathrm{R}_{\mathrm{q}}\right)^{60}$ showed an increase in surface roughness from $0.64 \mathrm{~nm}$ to $0.79 \mathrm{~nm}$ for the APTES-functionalized surface relative to the bare (non-functionalized one) (Supporting information Figure S2a, b and Figure S3a, b respectively). Similarly, analysis of the arithmetic average of the absolute values of the surface height deviations measured from the mean plane (i.e., $\left.R_{a}\right)^{60}$ showed an increase in the surface roughness from $0.78 \mathrm{~nm}$ to $0.99 \mathrm{~nm}$ for the APTES functionalized surface again, relative to the bare (non-functionalized one). 
Complementary, $\mathrm{R}_{\mathrm{q}}$ and $\mathrm{R}_{\mathrm{a}}$ for the APTES-glutaraldehyde-anti-kinesin antibodies functionalized surface were $1.12 \mathrm{~nm}$ and $1.54 \mathrm{~nm}$ (Supporting information Figure S4 a, b respectively).

a)

Substrate

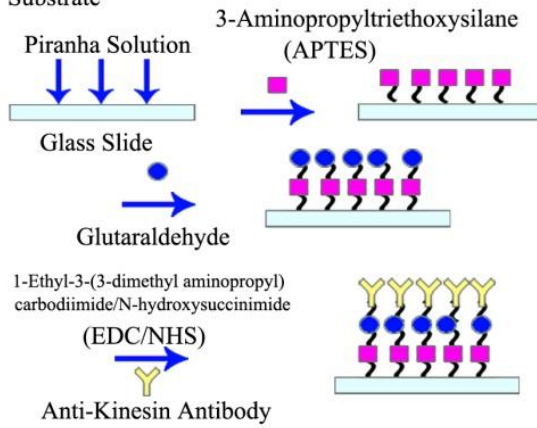

c)

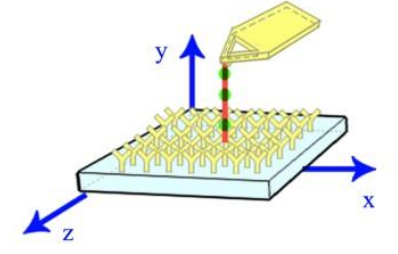

b)
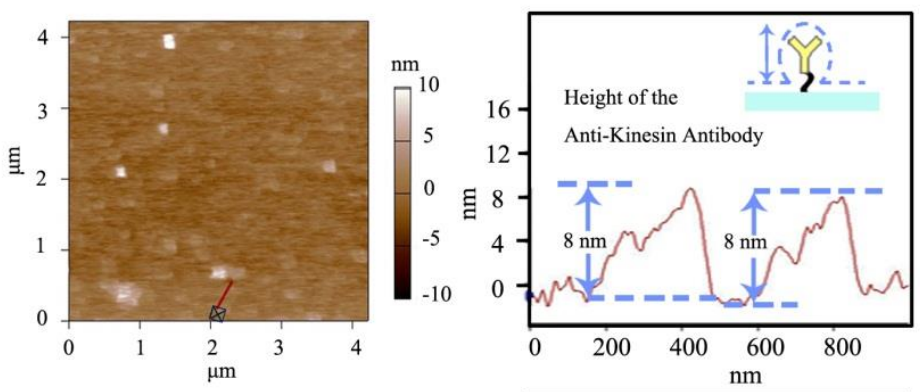

d)

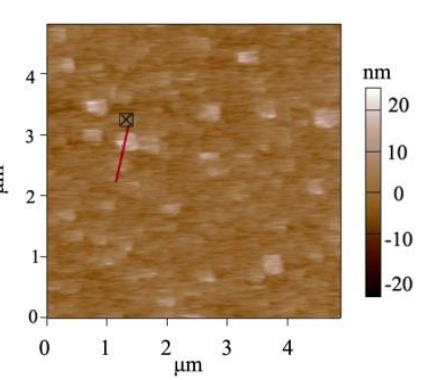

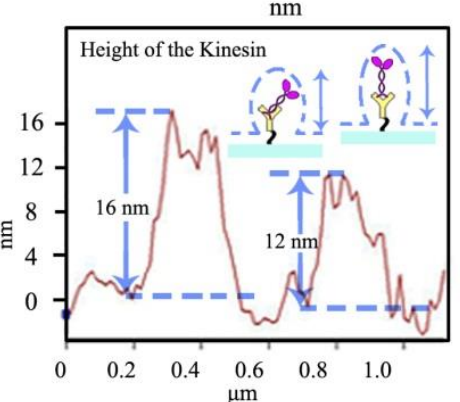

Figure 3: a) Schematic of the glass multi-step functionalization process through zero-length chemistry and antibody binding through APTES-based reaction. b) Representative AFM morphology of anti-kinesin antibodies coated surfaces and the statistical variation in such height distribution (as depicted on the red line). c) Schematic of single kinesin molecules written by the affixed microtubule pen. d) Representative AFM morphology and distribution of single kinesin molecules written by the affixed microtubule pen and the statistical variation in the height distribution of kinesin immobilized onto anti-kinesin antibody (as depicted on the red line). The possible geometries and resulting anisotropies are discussed relative to the individual molecule packing at the user-functionalized interface.

In contact mode AFM, the anti-kinesin antibodies functionalized surface appeared generally smooth with isolated anisotropies of dots-like geometries (Figure 3b). Surface analysis of such dots geometries revealed heights between 2 to $8 \mathrm{~nm}$ (70 geometries evaluated). The recorded anisotropy is due to binding geometries that the antibodies and APTES could assume at the mica. Specifically, previous reports have showed that APTES could assume 5 different orientations (i.e. "single siloxane", "double siloxane", "triple siloxane", "polymer" and "hydrogen bond" respectively, Supporting information Figure S5) 
when immobilized onto a glass $\operatorname{slide}^{36}$, with individual orientation impacting the subsequent height distribution of the immobilized molecule. Complementary, previous analysis showed immobilized antibodies could also assume different orientations at a support interface from the "End-on Fab-up", to the "End-on Fab-down", "Side-on" and/or "Flat-on" geometry with such molecular packing not only affecting individual antibody orientation and height at a support interface, but also its individual rate and ability to bind other ligands ${ }^{37}$.

The dispensing and capturing, a.k.a kinesin writing, was subsequently proposed as a kinesin molecule stepping off the inked microtubule when the AMPPNP was exchanged with ATP chemical energy to be captured by the proximal anti-kinesin antibodies (Figure 3c and Supporting Information Scheme S1). In particular, previous analysis have showed that kinesin molecules could walk processively with $8 \mathrm{~nm}$ steps and each step under one ATP hydrolysis event to subsequently dissociate once it reaches the end of an immobilized microtubule ${ }^{61}$. Further, previous research has also showed that the stepping length of a single kinesin molecule is influenced by experimental conditions such as the ATP concentration, salinity or load size just to name a few. In particular, Thorn et. al., ${ }^{62}$ showed that single wild type kinesin had a stepping length of $1.0 \pm 0.2 \mu \mathrm{m}$ and $0.7 \pm 0.1 \mu \mathrm{m}$, while single H1Q mutant kinesin showed a stepping length of $5.8 \pm 1.6 \mu \mathrm{m}$ and $1.5 \pm 0.5 \mu \mathrm{m}$ before and after treatment with subtilisin respectively. Further, Nishiyama et al., ${ }^{63}$ showed that the stepping length and velocity of the kinesin is a function of load. Authors estimated that a single kinesin at zero load had a run length of about $3.6 \mu \mathrm{m}$, at a velocity of about $930 \mathrm{~nm} / \mathrm{s}$. The run length and velocity decreased to $0.2 \mu \mathrm{m}$ and $230 \mathrm{~nm} / \mathrm{s}$ for a $3.8 \mathrm{pN}$ load, and to zero when the load increased to $7.6 \mathrm{pN}$. Lastly, intramolecular tension generated by the“neck linkers" was also shown to affect the kinesin stepping length ${ }^{64}$. For instance, Yildiz et al., ${ }^{64}$ presented a wild type kinesin that had a run length of about $2 \mu \mathrm{m}$ while the run length varied from about 1.5 $\mu \mathrm{m}$ to about $2.3 \mu \mathrm{m}$ for a kinesin with different neck linkers.

Considering that orientation of both APTES and anti-kinesin antibodies leads to anisotropic geometries ${ }^{36,65}$, we further hypothesized that any kinesin dispensed from the microtubule pen to be written onto the anti-kinesin antibodies would not only lead to an additional increase in local surface roughness at its place of binding to the anti-kinesin antibodies, but it would also have a locally distributed geometry that will mimic the initial surface dot-like one. Indeed, for the first, kinesin writing led to an additional increase in surface roughness, i.e., from $1.12 \mathrm{~nm}$ to to $4.00 \mathrm{~nm}$ for $\mathrm{R}_{\mathrm{q}}$ and from $1.54 \mathrm{~nm}$ to $5.02 \mathrm{~nm}$ for $\mathrm{R}_{\mathrm{a}}$ for the APTES-glutaraldehyde-anti-kinesin antibodies-kinesin relative to the 
APTES-glutaraldehyde-anti-kinesin antibodies functionalized surface (Figure 3d, Supporting information Figure S6 a, b). Kinesin written onto the anti-kinesin antibodies assumed a dot-like conformation of recorded heights (horizontal diameter) between 4 17 nm (109 molecules evaluated; Figure 3d). Results are supported by previous analysis that showed that immobilized kinesin could assume heights of $10.0 \pm 1.8 \mathrm{~nm}$ for long or $8.6 \pm 2.1 \mathrm{~nm}$ for short a short diameter ${ }^{66}$. Others have also showed using electron micrographs globular distributions of kinesins with a diameter of 9 10 $\mathrm{nm}^{67}$.

The height variation observed for the written kinesin, similarly to the height variation of the antibodies/APTES surfaces, could be due to the packing of the individual kinesin molecule as resulted from its defined protein-protein interaction. Specifically, since kinesin could assume either a stand up or a lay on geometry ${ }^{68}$, kinesin-antibody conjugates could also assume different geometries. Specifically, Figure 4a shows the multi-Guassian fitings of the height distributions for the two functionalized surfaces, namely anti-kinesin antibodies and kinesin-anti-kinesin antibodies respectively. For the anti-kinesin antibodies, peaks heights varied from $2 \mathrm{~nm}$ to $17 \mathrm{~nm}$ with most of them showing heights smaller than $11 \mathrm{~nm}$. The blue solid line resulted from the guassian fit identified the average height of anti-kinesin antibody of $5.54 \pm 2.91 \mathrm{~nm}$ and two peak distributions at $4 \mathrm{~nm}$ and $8 \mathrm{~nm}$ respectively. These could be presumablly associated with single anti-kinesin antibody with different orientations and/or anti-kinesin antibody conjugates respectively. For the kinesin-anti-kinesin antibodies, the heights changed from $4 \mathrm{~nm}$ to $29 \mathrm{~nm}$ with a dominant regime in the 4 20 $\mathrm{nm}$ range. The red solid line showed the gaussian fit results and identified an average height of kinesin-anti-kinesin antibodies of $11.26 \pm 5.73 \mathrm{~nm}$ with two peak distributions at $8 \mathrm{~nm}$ and 12 $\mathrm{nm}$ respectively. Such peaks may be associated with the height of single kinesin-anti-kinesin antibody pair and/or kinesin-anti-kinesin antibody conjugates respectively. In addition, there is an overlap area (between $6 \sim 8 \mathrm{~nm}$ ) of the height distributions for both anti-kinesin antibodies and kinesin-anti-kinesin antibodies respectively. In this area, the height of the anti-kinesin antibodies could be mainly measured on anti-kinesin antibody conjugates or single anti-kinesin antibody with a stand orientation (both "End-on Fab-up" and "End-on Fab-down" orientations). Complementary, the heights of the kinesin-anti-kinesin antibodies could be associated with single kinesin-anti-kinesin antibody pairs with a lay orientation (kinesin bound with an antibody in "Side-on" or "Flat-on" orientation, single anti-kinesin antibodies and kinesin with a stand orientation (both "Side-on" and "Flat-on" orientations).

Further, we hypothesized that longer contact time between the affixed biopen and the 
anti-kinesin functionalized surface will lead to writing capabilities of individual kinesin molecules that mimic the geometry of the biopen. Our analysis showed that indeed, allocating more time for writing, leads to controllable geometries of kinesin molecules on the user-functionalized surface. Specifically, upon increasing the writing time (i.e., the time allocated for the affixed pen to be in close proximity of the anti-kinesin antibody coated surfaces), circular patterns of kinesin were obtained (Figure 4b). Such circle-like structure was presumably formed by kinesin molecules walking, dissociating and binding onto the anti-kinesin antibody surfaces from the different profilaments they occupied on the affixed pen. Such assumption is based on previous research that showed single kinesin molecules walk processively on individual microtubule protofilaments towards its positive end ${ }^{33}$. This assumption is also supported by our own analysis of the changes in the surface distribution upon longer writing time. Specifically, the representative height variations observed for the written kinesin in the circle patterns were about $9 \mathrm{~nm}$ and $15 \mathrm{~nm}$ respectively. The $R_{q}$ and $R_{a}$ were $4.38 \pm 0.84 \mathrm{~nm}$ and $2.92 \pm 0.76 \mathrm{~nm}$ for the area with circle pattern and $1.92 \pm 0.34 \mathrm{~nm}$ and $1.52 \pm 0.13 \mathrm{~nm}$ for the area without the pattern.

The strategy presented herein is the first demonstration of a functional biological pen with the ability to write single molecules on user-functionalized surfaces. Such pen could in the future be used for writing soft molecules but also modified inks. For instance, one could envision functionalizing kinesin with nanosphere ${ }^{69}$ or CdSe quantum dot nanocomposites ${ }^{70}$ for writing of such individual inks in a parallel yet independent manner, all with sub-nm resolution dictated by a single kinesin step of $8 \mathrm{~nm}$ and to be used for applications in electrocatalysis ${ }^{71}$, sensing ${ }^{72}$ and bioimaging ${ }^{73}$. Moreover, one could envision controlling the ability to dispense such ink by controlling the energy provided at a given time. Indeed, previous analysis ${ }^{74}$ has showed that controlling ATP concentrations could lead to differences in the kinesin speed ( from $10 \mathrm{~nm} / \mathrm{s}$ for $1 \mu \mathrm{M}$ to $550 \mathrm{~nm} / \mathrm{s}$ for $1000 \mu \mathrm{M}$ for instance). Further by controlling the affixing of the pen at the tip one could possibly control the holding position and the dispensing ability/efficiency. For instance, using anti-gamma tubulin antibodies would ensure only immobilization of the minus end of the microtubule ${ }^{75}$ with the plus end to be always exposed and in close proximity of the surface for continuous writing of inked kinesins ${ }^{75}$. Complementary, by using contact mode AFM association and dissociation events between the antibodies functionalized kinesin and the kinesin molecule could be possibly evaluated to differentiate multi or single bonds formed at such interfaces as well as their energy landscapes and their changes based on ligand structural arrangement at nanointerfaces and how 
these depend on the specificity of the ligand-recognition reaction ${ }^{37}$. Lastly, it is envisioned that such a pen could potentially have the ability for parallelization while still providing individual writing nanometer characteristics. Specifically, considering that a microtubule is formed from 13 independent protofilaments ${ }^{76}$ with each one of the possible filaments to be used by multiple inks ${ }^{77}$ at a given time and for multiple inking cycles, one could envision the ability for controlled nanomanufacturing with ultra high resolution of a multitude of inks for applications in nanoelectronics, pick and place nanorobots or synthetic biology.

a)

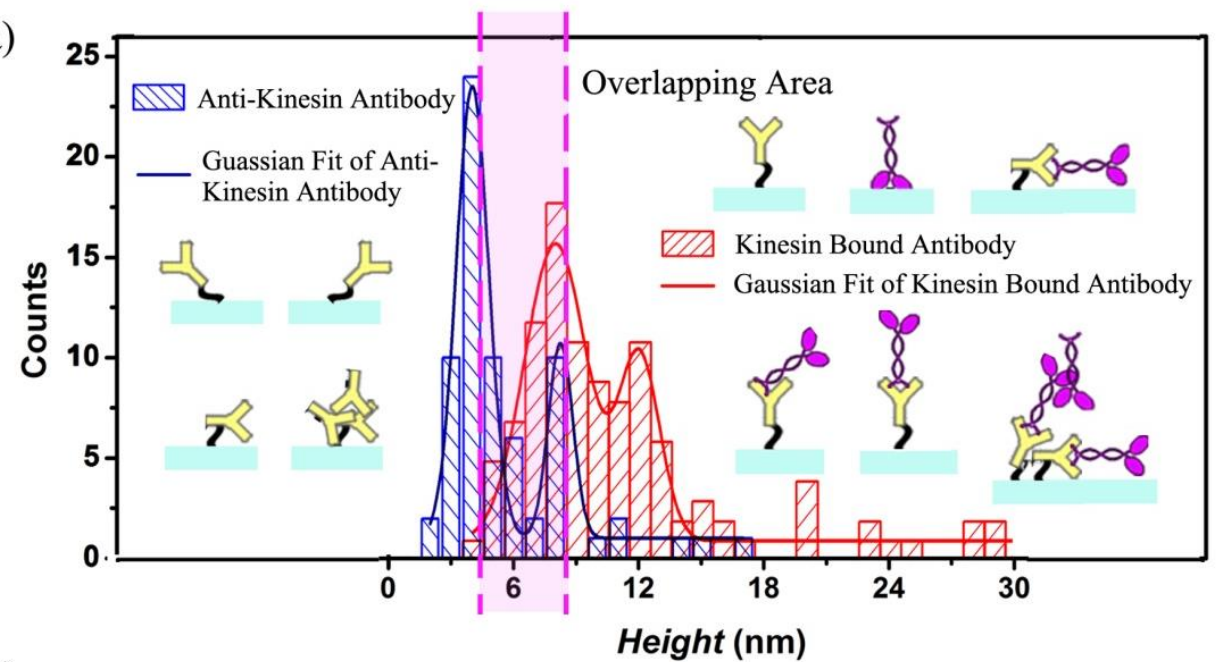

b)

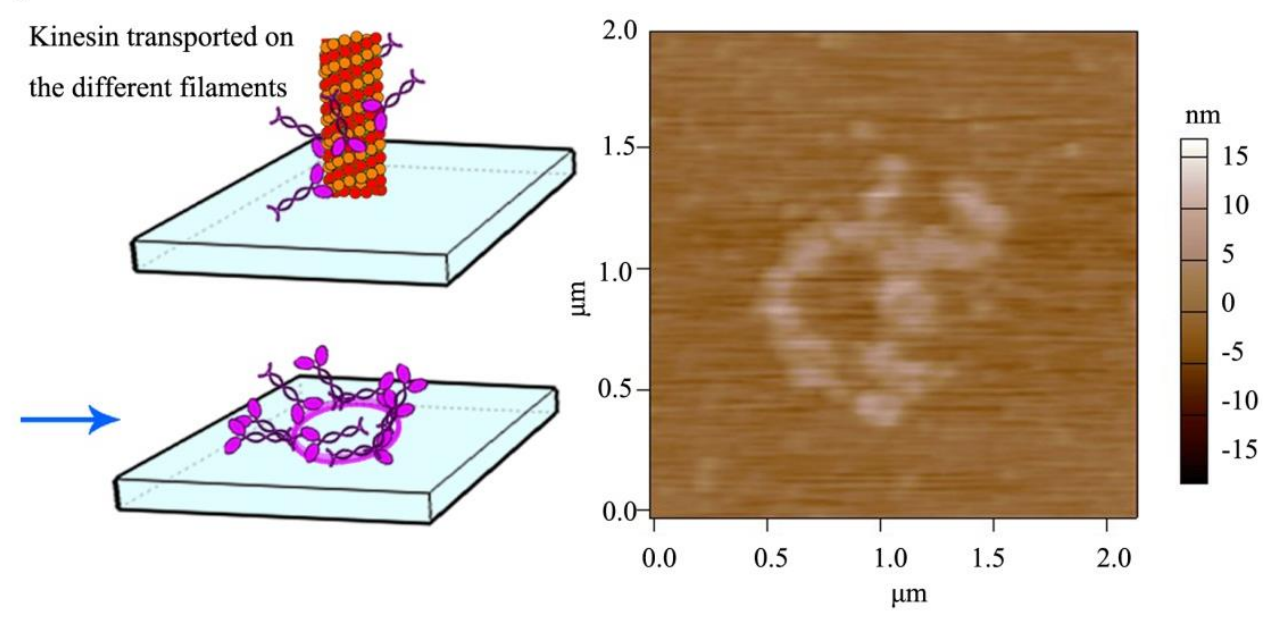

Figure 4: a) The statistical variation in the height distribution of kinesin immobilized onto anti-kinesin antibody and control of height distribution of anti-kinesin antibody; 69 anti-kinesin antibody and 104 kinesin bound anti-kinesin antibody were evaluated in the height distributions. The possible geometries and resulting anisotropies are discussed relative to the individual molecule packing at the user-functionalized interface. b) Kinesin walks on the different filaments of the microtubule and written onto anti-kinesin antibody functionalized surfaces (left) to be leading to circular morphological patterns (right). 


\section{Conclusions}

Our study successfully produced a bio-pen using an AFM inert tip and molecular structures isolated from the cell. Our results showed that the ink (single kinesin molecules) could be immobilized onto a biological pen (microtubule affixed at the AFM tip) and autonomously written onto user-immobilized anti-kinesin antibody coated substrate, all under the biochemical energy of ATP. The obtained biological pen has the ability to create different nanostructures on a user-functionalized substrate to be used in applications from biosensing, high-throughput screening to nanomanipulation and structure formation. 


\section{References:}

1 Su, J., Goldberg, A. F. G. \& Stoltz, B. M. Label-free detection of single nanoparticles and biological molecules using microtoroid optical resonators. Light: Science \&Amp; Applications 5, e16001, (2016).

2 Gao, M. 3D Printing of Nanostructures. Advanced Nano Deposition Methods, 209-221 (2016).

3 Yang, S., Dai, X., Stogin, B. B. \& Wong, T.-S. Ultrasensitive surface-enhanced Raman scattering detection in common fluids. Proceedings of the National Academy of Sciences 113, 268-273 (2016).

4 Riba, J. et al. Molecular Genetic Characterization of Individual Cancer Cells Isolated via Single-Cell Printing. PloS one 11, e0163455 (2016).

5 Chen, Y. Nanofabrication by electron beam lithography and its applications: a review. Microelectronic Engineering 135, 57-72 (2015).

6 Sumaru, K., Takagi, T., Morishita, K., Satoh, T. \& Kanamori, T. Fabrication of pocket-like hydrogel microstructures through photolithography. Soft Matter (2018).

7 Brinkmann, F. et al. Interdigitated multicolored bioink micropatterns by multiplexed polymer pen lithography. Small 9, 3266-3275 (2013).

8 Hamon, C. et al. Tunable Nanoparticle and Cell Assembly Using Combined Self Powered Microfluidics and Microcontact Printing. Advanced Functional Materials 26, 8053-8061 (2016).

9 Brown, K. A. et al. A cantilever-free approach to dot-matrix nanoprinting. Proceedings of the National Academy of Sciences 110, 12921-12924 (2013).

10 Hwang, J. K. et al. Direct nanoprinting by liquid-bridge-mediated nanotransfer moulding. Nature nanotechnology 5, 742 (2010).

11 Nam, H. et al. MoS2 transistors fabricated via plasma-assisted nanoprinting of few-layer MoS2 flakes into large-area arrays. ACS nano 7, 5870-5881 (2013).

12 Chmela, O. et al. in Nanotechnology VIII. 102480F (International Society for Optics and Photonics).

13 Feng, J., Tuominen, M. T. \& Rothstein, J. P. Hierarchical superhydrophobic surfaces fabricated by dual - scale electron - beam - lithography with well - ordered secondary nanostructures. Advanced Functional Materials 21, 3715-3722 (2011).

14 Valsecchi, C. et al. Low-Cost Leukemic Serum Marker Screening Using Large Area Nanohole Arrays on Plastic Substrates. ACS Sensors 1, 1103-1109, (2016). 
15 Temiz, Y., Lovchik, R. D. \& Delamarche, E. in Microchip Diagnostics (Springer, 2017).

16 Arango-Santander, S., Freitas, S. C., Pelaez-Vargas, A. \& García, C. in Key Engineering Materials. 264-268 (Trans Tech Publ).

17 Changhai, R., Jun, L., Shaorong, X. \& Yu, S. A review of non-contact micro- and nano-printing technologies. Journal of Micromechanics and Microengineering 24, 053001 (2014).

18 O'Connell, C., Higgins, M. J., Moulton, S. E. \& Wallace, G. G. Nano-bioelectronics via dip-pen nanolithography. Journal of Materials Chemistry C 3, 6431-6444 (2015).

$19 \mathrm{Xu}, \mathrm{S} . \& \mathrm{Liu}, \mathrm{G} .-\mathrm{y}$. Nanometer-scale fabrication by simultaneous nanoshaving and molecular self-assembly. Langmuir 13, 127-129 (1997).

20 Kufer, S. K., Puchner, E. M., Gumpp, H., Liedl, T. \& Gaub, H. E. Single-Molecule Cut-and-Paste Surface Assembly. Science 319, 594-596, science.1151424 (2008).

21 Son, J. Y., Shin, Y.-H., Ryu, S., Kim, H. \& Jang, H. M. Dip-pen lithography of ferroelectric PbTiO3 nanodots. Journal of the American Chemical Society 131, 14676-14678 (2009).

22 Corletto, A., Yu, L., Shearer, C. J., Gibson, C. T. \& Shapter, J. G. Direct - Patterning SWCNTs Using Dip Pen Nanolithography for SWCNT/Silicon Solar Cells. Small 14, 1800247 (2018).

23 Rath, P. et al. Diamond Nanophotonic Circuits Functionalized by Dip - pen Nanolithography. Advanced Optical Materials 3, 328-335 (2015).

24 Zhang, Y., Liu, C. \& Whalley, D. in Electronic Packaging Technology \& High Density Packaging, 2009. ICEPT-HDP'09. International Conference on. 497-503 (IEEE).

25 Ginger, D. S., Zhang, H. \& Mirkin, C. A. The Evolution of dip - Pen nanolithography. Angewandte Chemie International Edition 43, 30-45 (2004).

26 Salaita, K., Wang, Y. \& Mirkin, C. A. Applications of dip-pen nanolithography. Nature Nanotechnology 2, 145,.2007.39 (2007).

27 Brown, K. A., Eichelsdoerfer, D. J., Liao, X., He, S. \& Mirkin, C. A. Material transport in dip-pen nanolithography. Frontiers of Physics 9, 385-397, (2014).

28 Wei, X., Chen, Z., Tan, L., Lou, T. \& Zhao, Y. DNA-catalytically active gold nanoparticle conjugates-based colorimetric multidimensional sensor array for protein discrimination. Analytical chemistry 89, 556-559 (2016).

29 Pelton, R. Bioactive paper provides a low-cost platform for diagnostics. TrAC Trends 
in Analytical Chemistry 28, 925-942 (2009).

30 Wettstein, G., Bellaye, P., Micheau, O. \& Bonniaud, P. Small heat shock proteins and the cytoskeleton: an essential interplay for cell integrity? The international journal of biochemistry \& cell biology 44, 1680-1686 (2012).

31 Hayashi, K., Hasegawa, S., Sagawa, T., Tasaki, S. \& Niwa, S. Non-invasive force measurement reveals the number of active kinesins on a synaptic vesicle precursor in axonal transport regulated by ARL-8. Physical Chemistry Chemical Physics 20, 3403-3410 (2018).

32 Klinman, E. \& Holzbaur, E. L. Walking Forward with Kinesin. Trends in neurosciences 41, 555-556 (2018).

33 Seitz, A. \& Surrey, T. Processive movement of single kinesins on crowded microtubules visualized using quantum dots. The EMBO journal 25, 267-277 (2006).

34 Isojima, H., Iino, R., Niitani, Y., Noji, H. \& Tomishige, M. Direct observation of intermediate states during the stepping motion of kinesin-1. Nature chemical biology 12, 290 (2016).

35 Hiratsuka, Y., Tada, T., Oiwa, K., Kanayama, T. \& Uyeda, T. Q. Controlling the direction of kinesin-driven microtubule movements along microlithographic tracks. Biophysical Journal 81, 1555-1561 (2001).

$36 \mathrm{Hu}, \mathrm{X}$., Dong, C., Su, R., Xu, Q. \& Dinu, C. Z. Protein self-assembly onto nanodots leads to formation of conductive bio-based hybrids. Scientific reports 6, 38252 (2016).

$37 \mathrm{Hu}, \mathrm{X}$. \& Dinu, C. Z. Analysis of affinities between specific biological ligands using atomic force microscopy. Analyst 140, 8118-8126 (2015).

38 Dinu, C. Z. et al. Parallel Manipulation of Bifunctional DNA Molecules on Structured Surfaces Using Kinesin - Driven Microtubules. Small 2, 1090-1098 (2006).

39 Li, R., Ye, H., Zhang, W., Ma, G. \& Su, Y. An analytic model for accurate spring constant calibration of rectangular atomic force microscope cantilevers. Scientific reports 5, 15828 (2015).

40 Li, X., He, J., Zhang, W., Jiang, N. \& Li, D. Additive manufacturing of biomedical constructs with biomimetic structural organizations. Materials 9, 909 (2016).

41 Michalski, M. H. \& Ross, J. S. The shape of things to come: 3D printing in medicine. Jama 312, 2213-2214 (2014).

42 Ready, S. et al. in NIP \& Digital Fabrication Conference. 9-12 (Society for Imaging 
Science and Technology).

43 Chia, H. N. \& Wu, B. M. Recent advances in 3D printing of biomaterials. Journal of Biological Engineering 9, 4, (2015).

44 Benoit, M. P. \& Sosa, H. in Single Molecule Analysis 199-216 (Springer, 2018).

45 Yajima, J., Alonso, M. C., Cross, R. A. \& Toyoshima, Y. Y. Direct long-term observation of kinesin processivity at low load. Current biology 12, 301-306 (2002).

46 Migneault, I., Dartiguenave, C., Bertrand, M. J. \& Waldron, K. C. Glutaraldehyde: behavior in aqueous solution, reaction with proteins, and application to enzyme crosslinking. Biotechniques 37, 790-806 (2004).

47 Han, S.-W., Simona, P., Banu, M. \& Adachi, T. Real-time Monitoring of Changes in Microtubule Mechanical Properties in Response to Microtubule-Destabilizing Drug Treatment. Journal of nanoscience and nanotechnology 13, 2087-2090 (2013).

48 Schaap, I. A. T., Hoffmann, B., Carrasco, C., Merkel, R. \& Schmidt, C. F. Tau protein binding forms a $1 \mathrm{~nm}$ thick layer along protofilaments without affecting the radial elasticity of microtubules. Journal of Structural Biology 158, 282-292, (2007).

49 Munson, K. M., Mulugeta, P. G. \& Donhauser, Z. J. Enhanced mechanical stability of microtubules polymerized with a slowly hydrolyzable nucleotide analogue. $J$ Phys Chem B 111, 5053-5057, (2007).

50 Drechsler, H. \& McAinsh, A. D. Kinesin-12 motors cooperate to suppress microtubule catastrophes and drive the formation of parallel microtubule bundles. Proceedings of the National Academy of Sciences 113, E1635-E1644, (2016).

51 Abraham, Z., Hawley, E., Hayosh, D., Webster-Wood, V. A. \& Akkus, O. Kinesin and Dynein Mechanics: Measurement Methods and Research Applications. Journal of Biomechanical Engineering 140, 020805-020805-020811, (2018).

52 Carré, M. et al. Tubulin is an inherent component of mitochondrial membranes that interacts with the voltage-dependent anion channel. Journal of Biological Chemistry 277, 33664-33669 (2002).

53 Schaap, I. A. T., Carrasco, C., de Pablo, P. J. \& Schmidt, C. F. Kinesin walks the line: single motors observed by atomic force microscopy. Biophysical journal $\mathbf{1 0 0}$, 2450-2456, (2011).

54 Kacher, C. M. et al. Imaging microtubules and kinesin decorated microtubules using tapping mode atomic force microscopy in fluids. European Biophysics Journal 28, 611-620, (2000). 
$55 \mathrm{Hu}, \mathrm{X}$. et al. Biological Self-Assembly and Recognition Used to Synthesize and Surface Guide Next Generation of Hybrid Materials. ACS Applied Materials \& Interfaces 10, 28372-28381, (2018).

56 Seeger, M. A. \& Rice, S. E. Microtubule-associated protein-like binding of the kinesin-1 tail to microtubules. Journal of Biological Chemistry 285, 8155-8162 (2010).

57 Takahashi, Y. et al. Topographic imaging of convoluted surface of live cells by scanning ion conductance microscopy in a standing approach mode. Physical Chemistry Chemical Physics 12, 10012-10017 (2010).

58 Takahashi, Y. et al. Topographical and electrochemical nanoscale imaging of living cells using voltage-switching mode scanning electrochemical microscopy. Proceedings of the National Academy of Sciences 109, 11540 (2012).

59 Boeri Erba, E., Klein, P. A. \& Signor, L. Combining a NHS ester and glutaraldehyde improves crosslinking prior to MALDI MS analysis of intact protein complexes. Journal of Mass Spectrometry 50, 1114-1119 (2015).

60 Rao, C. M., Venkatasubbaiah, K. \& Rao, K. J. Experimental Investigation of Surface Roughness Characteristics $\mathrm{Ra}, \mathrm{Rq}$ and $\mathrm{Rz}$. International Journal of Hybrid Information Technology 9, 373-388 (2016).

61 Mickolajczyk, K. J. et al. Kinetics of Nucleotide-Dependent Structural Transitions in the Kinesin-1 Hydrolysis Cycle. Biophysical Journal 110, 193a (2016).

62 Thorn, K. S., Ubersax, J. A. \& Vale, R. D. Engineering the Processive Run Length of the Kinesin Motor. The Journal of Cell Biology 151, 1093-1100, (2000).

63 Nishiyama, M., Higuchi, H. \& Yanagida, T. Chemomechanical coupling of the forward and backward steps of single kinesin molecules. Nature Cell Biology 4, 790, (2002).

64 Yildiz, A., Tomishige, M., Gennerich, A. \& Vale, R. D. Intramolecular Strain Coordinates Kinesin Stepping Behavior along Microtubules. Cell 134, 1030-1041, (2008).

65 Satija, J., Karunakaran, B. \& Mukherji, S. A dendrimer matrix for performance enhancement of evanescent wave absorption-based fiber-optic biosensors. RSC Advances 4, 15841-15848 (2014).

66 Hirokawa, N. et al. Submolecular domains of bovine brain kinesin identified by electron microscopy and monoclonal antibody decoration. Cell 56, 867-878 (1989). 
67 Scholey, J. M., Heuser, J., Yang, J. T. \& Goldstein, L. S. Identification of globular mechanochemical heads of kinesin. Nature 338, 355 (1989).

68 Jung, Y., Jeong, J. Y. \& Chung, B. H. Recent advances in immobilization methods of antibodies on solid supports. Analyst 133, 697-701, (2008).

69 Doot, R. K., Hess, H. \& Vogel, V. Engineered networks of oriented microtubule filaments for directed cargo transport. Soft Matter 3, 349-356 (2007).

70 Bachand, G. D. et al. Assembly and Transport of Nanocrystal CdSe Quantum Dot Nanocomposites Using Microtubules and Kinesin Motor Proteins. Nano Letters 4, 817-821, (2004).

$71 \mathrm{Gu}$, L. et al. Microwave-assisted synthesis of nanosphere-like NiCo2O4 consisting of porous nanosheets and its application in electro-catalytic oxidation of methanol. Journal of Power Sources 261, 317-323 (2014).

72 Zhang, R. \& Chen, W. Nitrogen-doped carbon quantum dots: Facile synthesis and application as a "turn-off" fluorescent probe for detection of $\mathrm{Hg}^{2+}$ ions. Biosensors and Bioelectronics 55, 83-90 (2014).

73 Lv, R. et al. Hollow Structured Y2O3: Yb/Er-Cu x S Nanospheres with Controllable Size for Simultaneous Chemo/Photothermal Therapy and Bioimaging. Chemistry of Materials 27, 483-496 (2015).

74 Asenjo, A. B. \& Sosa, H. A mobile kinesin-head intermediate during the ATP-waiting state. Proceedings of the National Academy of Sciences 106, 5657-5662, (2009).

75 Akhmanova, A. \& Hoogenraad, C. C. Microtubule minus-end-targeting proteins. Current Biology 25, R162-R171 (2015).

76 Kollman, J. M. et al. Ring closure activates yeast $\gamma$ TuRC for species-specific microtubule nucleation. Nature Structural and Molecular Biology 22, 132 (2015).

77 Weinger, J. S., Qiu, M., Yang, G. \& Kapoor, T. M. A nonmotor microtubule binding site in kinesin-5 is required for filament crosslinking and sliding. Current Biology 21, 154-160 (2011). 


\section{Supporting Information}

\section{Strategy for printing single kinesin molecules on functionalized surfaces}

The process of microtubule's controlled inking and affixing allow for regular geometries/ patterns of kinesin to be written on anti-kinesin antibodies user-functionalized substrates. The strategy is summarized in Scheme S1 include below.

a)

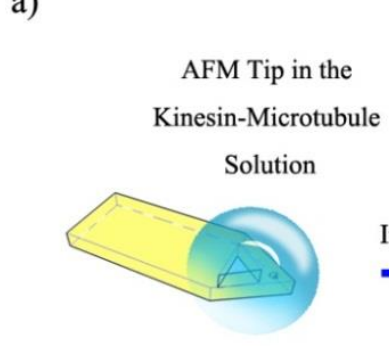

d)

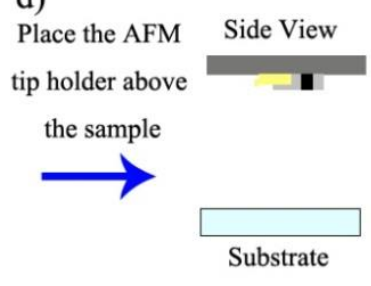

b)

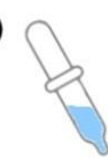

Wash with BRB80 buffer

containing $10 \mu \mathrm{M}$ paclitaxel
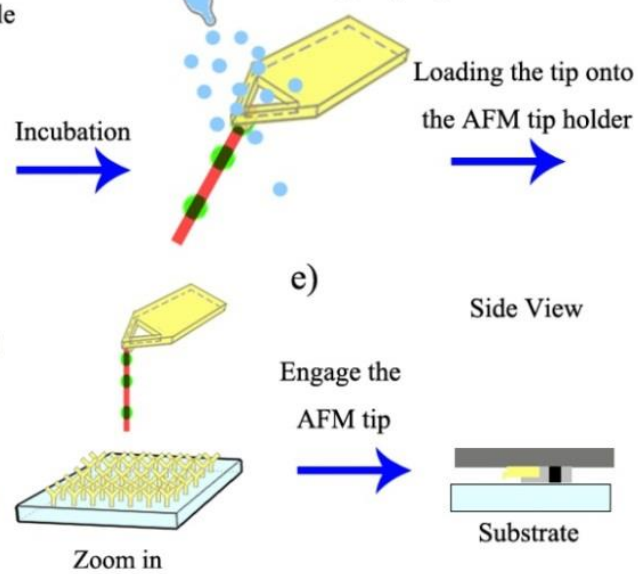

e)

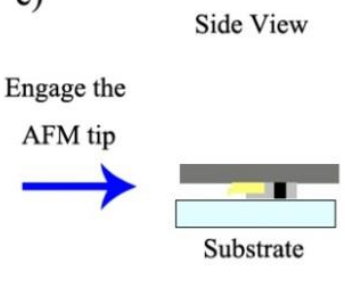

c)

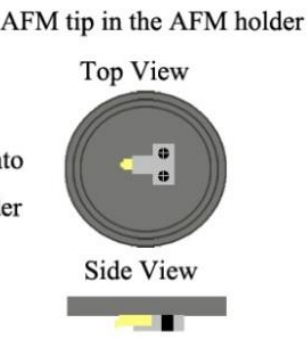

AFM tip in the AFM holder

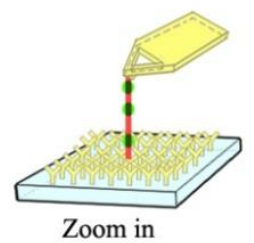

Scheme S1: Inking and AFM affixing of the biopen. Upon the biopen fabrication and affixing onto the AFM tip, the tip is brought in close proximity to the anti-kinesin functionalized surfaces to allow single kinesin molecule printing.

\section{Length of the microtubule}

Microtubule length was evaluated using optical microscopy. For this, microtubules were first synthesized from free tubulin suspended in a microtubule polymerization solution according to established protocol ${ }^{3}$. Briefly, the polymerization solution was obtained by vortexing $5 \mu \mathrm{L} 100 \mathrm{mM} \mathrm{MgCl}_{2}$, with $6 \mu \mathrm{L}$ dimethyl sulfoxide (DMSO, 99.7\%, Fisher Scientific, USA), $5 \mu \mathrm{L} 25 \mathrm{mM}$ guanosine-5'-triphosphate (GTP, Sigma, USA) and $9 \mu \mathrm{L}$ BRB80 buffer (formed from a mixture of $80 \mathrm{mM}$ piperazine-N,N'-bis(2-ethanesulfonic acid buffer, $1 \mathrm{mM} \mathrm{MgCl} 2$ and $1 \mathrm{mM}$ ethylene glycol tetraacetic acid (EGTA), $\mathrm{pH}$ 6.8; all reagents were purchased from Fisher Scientific, USA). To initiate microtubule polymerization, $2.5 \mu \mathrm{L}$ polymerization solution was mixed with $10 \mu \mathrm{L}$ of $4 \mathrm{mg} / \mathrm{mL}$ biotin and rhodamine labeled tubulin (Cytoskeleton Inc, USA) and the mixture was incubated at $37{ }^{\circ} \mathrm{C}$ for $30 \mathrm{~min}$. To stabilize the resulting microtubules, the solution was dispersed in $1 \mathrm{~mL}$ BRB80 buffer 
containing $10 \mu \mathrm{M}$ paclitaxel (Fisher Scientific, USA). The stabilized rhodamine labeled microtubules were analyzed under a 100 x objective $(\mathrm{NA}=1.4)$ on an MFP-3D Bio containing an integrated Nikon (Asylum/ Nikon, USA) and under the DAPI filter and an exposure time of $8.3 \mathrm{~s}$. Average length distributions were $18.94 \mathrm{~nm} \pm 8.41 \mathrm{~nm}$ (210 microtubule analyzed).

\section{Kinesin in vitro stepping assay}

Kinesins' ability to bind and step off the lab-synthesized microtubules was also evaluated. First, kinesin ability to bind to the microtubules was evaluated by mixing $8 \mu \mathrm{L}$ of $10 \mu \mathrm{g} / \mathrm{mL}$ of kinesin expressed in the lab (see Materials and Methods section of the manuscript) with 2 $\mu \mathrm{L} 20 \mathrm{mM}$ adenylyl-imidodiphosphate (AMP-PNP, Sigma, USA) and incubating the mixture for $1 \mathrm{~h}$ at $4{ }^{\circ} \mathrm{C}$. The mixture was subsequently mixed with $20 \mu \mathrm{L}$ microtubules (prepared as described in the manuscript in the Materials and Methods section) and $10 \mu \mathrm{L}$ of BRB80 buffer containing $10 \mu \mathrm{M}$ paclitaxel; resulting solution was incubated for $30 \mathrm{~min}$ at room temperature. Upon incubation, the unbound kinesin was separated in the supernatant by using an Allegra 64R centrifuge (Beckman Coulter, USA) and 30,000 rpm spinning for $10 \mathrm{~min}$.

Secondly, kinesin stepping off the microtubule was evaluated upon suspending the inked microtubule (i.e., inked biopen) as indicated in the Schematic 1 above and using an ATP-based assay ${ }^{4}$. For this, $100 \mu \mathrm{L}$ gliding solution (BRB80 buffer containing 1mM MgATP, $20 \mathrm{mM}$ D-glucose; both reagents were from Fisher Scientific, USA), $0.02 \mathrm{mg} / \mathrm{mL}$ glucose oxidase, $0.8 \mathrm{mg} / \mathrm{mL}$ catalase (both reagents from Sigma, USA) and 0.5\% $\beta$-mercaptoethanol (Fisher Scientific, USA) were added onto the tip functionalized with the kinesin-inked microtubule and incubated for $5 \mathrm{~min}$ at room temperature. The writing process was observed using a fluorescent microscope and a $100 \mathrm{x}$ objective $(\mathrm{NA}=1.4)$ under the DAPI and GFP filters and under an exposure time of $8.3 \mathrm{~s}$. Other representative images of stepping assay are included below. 


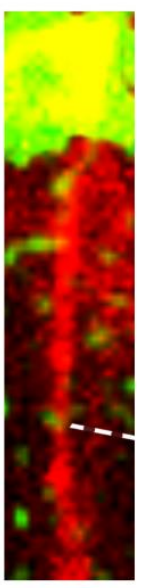

$20.5 \mathrm{~s}$

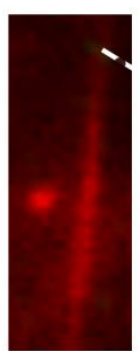

$14.76 \mathrm{~s}$

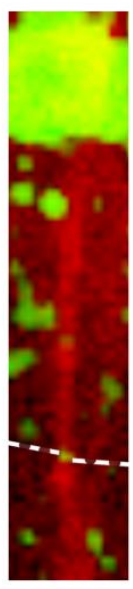

$21.9 \mathrm{~s}$

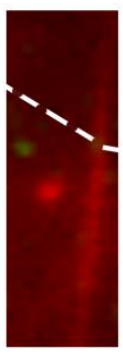

$15.48 \mathrm{~s}$

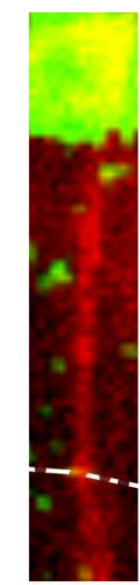

$22.3 \mathrm{~s}$

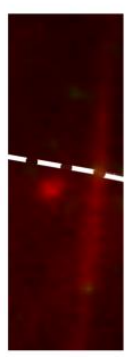

$16.2 \mathrm{~s}$
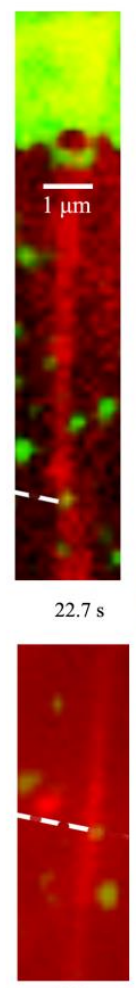

$17.0 \mathrm{~s}$
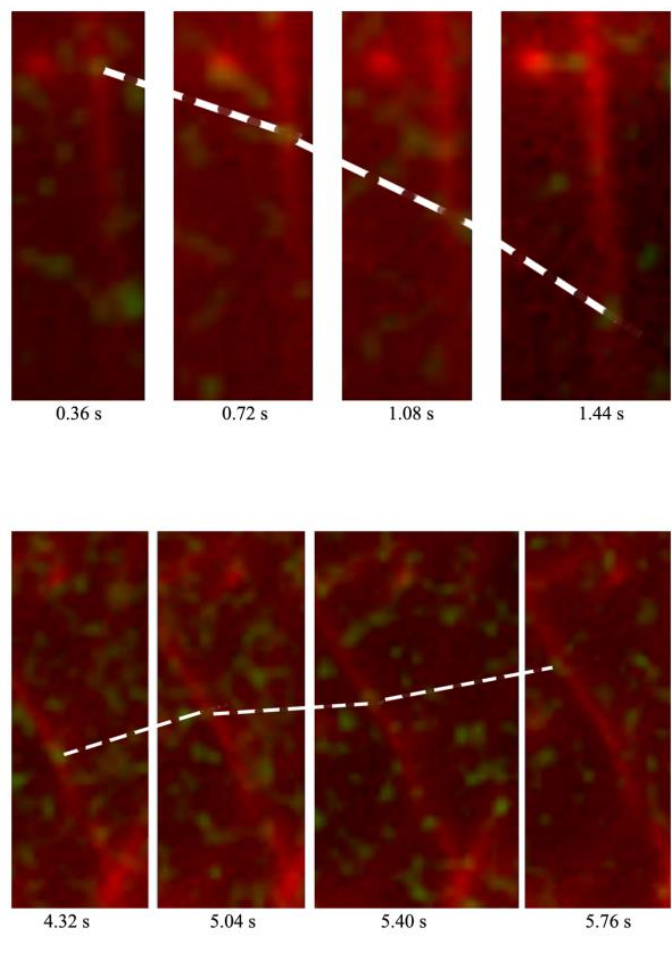

Figure S1: Time frame fluorescence images of the stepping assay from parallel/ individual experiments. Kinesin (green labeled) is shown moving on the immobilized microtubule (red).

\section{Calculations of the roughness factors $R_{q}$ and $R_{a}$}

Roughness factors $R_{q}$ and $R_{a}$ were defined as the root mean square average of height deviation (as taken from the mean image data plane) and the arithmetic average of absolute values of the surface height deviations (as measured from the mean plane) respectively, and were calculated using equations 1 and 2 below:

$$
\begin{aligned}
& R_{q}=\sqrt{\frac{1}{l} \int_{0}^{l} \mathrm{Z}^{2}(x) d x} \quad \text { (Eq.1), } \\
& R_{a}=\frac{1}{l} \int_{0}^{l}|Z(x)| d x
\end{aligned}
$$

Herein 1 is the total sample length and $\mathrm{Z}$ is the sample height ${ }^{1}$.

\section{Atomic Force Microscopy (AFM) morphology analyses}

\section{a) Clean glass substrate}

The morphology of a glass slide was evaluated using contact mode AFM and an MFP-3D Bio (Asylum, USA). For this, the glass slide $(\mathrm{d}=25 \mathrm{~mm}$, Corning, USA) was first 
ultrasonicated in DI water, 99\% ethanol (90\%, Fisher Scientific, USA), and again in DI water, with $30 \mathrm{~min}$ for each of the sonication window. Secondly, the slide was dried under vacuum for 1 day and subsequently exposed to UV light for $30 \mathrm{~min}$. The morphology of the glass slide was evaluated by contact mode AFM in liquid. For the AFM analyses, the cantilever's spring constant was calibrated using the thermal noise method; the scan rate of the tip was fixed at $0.5 \mathrm{~Hz}$ for all the experiments. At least 6 samples have been investigated to collect individual morphologies and results are shown in Figure $\mathbf{S 1} . \mathrm{R}_{\mathrm{q}}$ and $\mathrm{R}_{\mathrm{a}}$ of the clean glass substrate were calculated as listed above to result in 0.64 and $0.78 \mathrm{~nm}$ respectively.
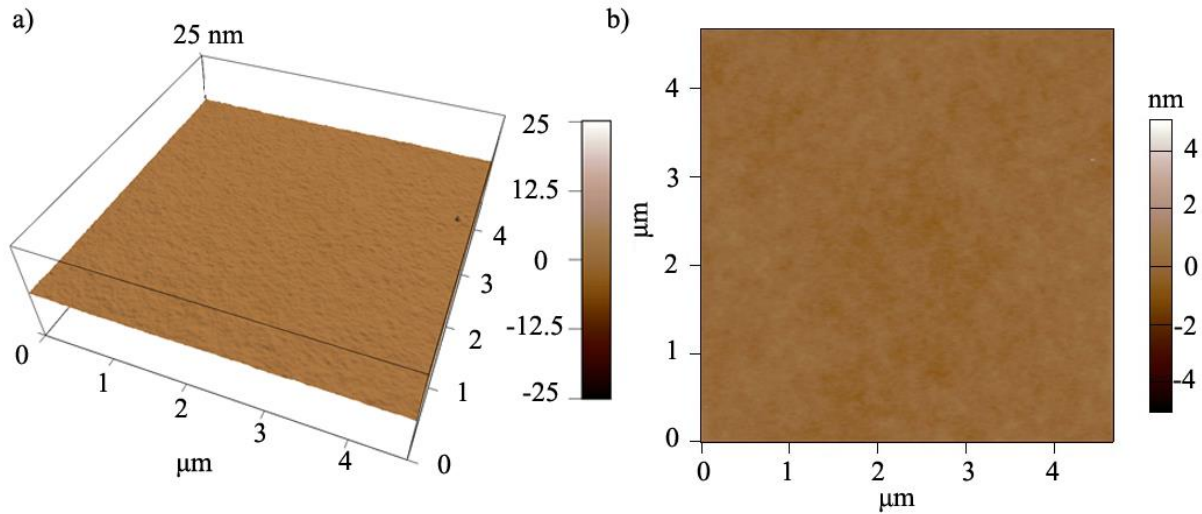

Figure S2: (a) Representative 3D AFM morphology analysis of a clean glass substrate. (b) Representative 2D AFM morphology analysis of a clean glass substrate.

\section{b) Functionalized glass surface}

The morphology of the 3-aminopropyltriethoxysilane (APTES) functionalized surface was also evaluated according to the protocol described above. Herein the APTES functionalization was obtained by immersing the clean glass in $1 \mathrm{ml}$ of $5 \%$ APTES in toluene (99.5\%) with incubation at room temperature for $1 \mathrm{~h}$. Upon time elapsed, the slide was washed thoroughly with DI water, toluene and again DI water to remove any unbound APTES. $R_{q}$ and $R_{a}$ of the APTES functionalized substrate were calculated from the Figure $\mathbf{S} 2$ and equaled to $0.79 \mathrm{~nm}$ and $0.99 \mathrm{~nm}$ respectively. 
a)

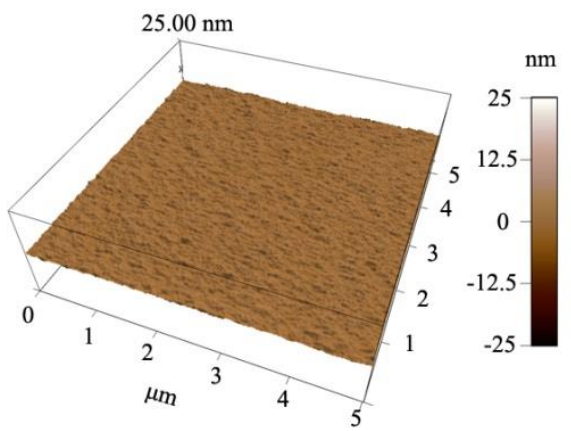

b)

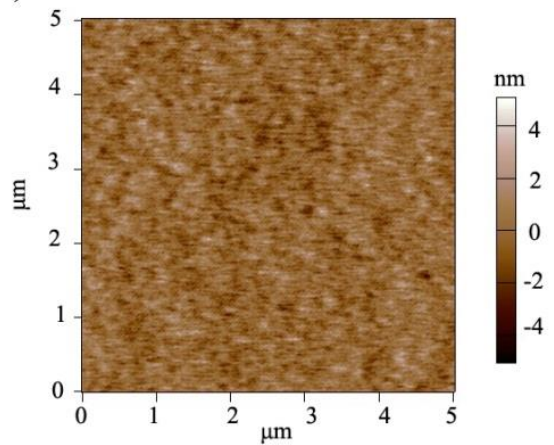

Figure S3: (a) Representative 3D AFM morphology of the APTES functionalized substrate surface. (b) Representative 2D AFM morphology of the APTES functionalized substrate surface.

\section{c) APTES-glutaraldehyde-anti-kinesin antibodies functionalized surface}

The morphology of the APTES-glutaraldehyde-anti-kinesin antibodies functionalized surface was also evaluated according to the protocol described above. Herein the glutaraldehyde-anti-kinesin antibodies functionalization was obtained upon immersing the APTES functionalized glass slide into $1 \mathrm{~mL} 5 \%$ glutaraldehyde in $0.2 \mathrm{M} \mathrm{pH} 9.0$ Tris-buffered saline (TBS) at room temperature, for $1 \mathrm{~h}$, with shaking at $200 \mathrm{rpm}$. Subsequently the slide was extensively rinsed with TBS, activated for $15 \mathrm{~min}$ in a $1 \mathrm{~mL} 160 \mathrm{mM}$ 1-Ethyl-3-(3-dimethylaminopropyl)carbodiimide and $80 \mathrm{mM}$ N-Hydroxysuccinimide (NHS)

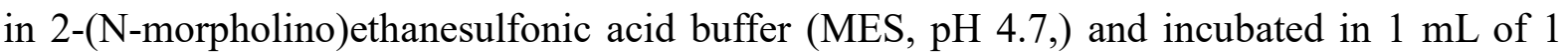
$\mathrm{mg} / \mathrm{mL}$ anti-kinesin antibody in BRB80 at room temperature and $200 \mathrm{rpm}$ for $3 \mathrm{~h}$ respectively. Upon incubation, the functionalized glass slide was rinsed thoroughly with BRB80 to remove loosely bound antibodies. $\mathrm{R}_{\mathrm{q}}$ and $\mathrm{R}_{\mathrm{a}}$ of the APTES-glutaraldehyde-anti-kinesin antibodies functionalized substrate were calculated and equaled values of 1.12 and $1.54 \mathrm{~nm}$ respectively. 
a)

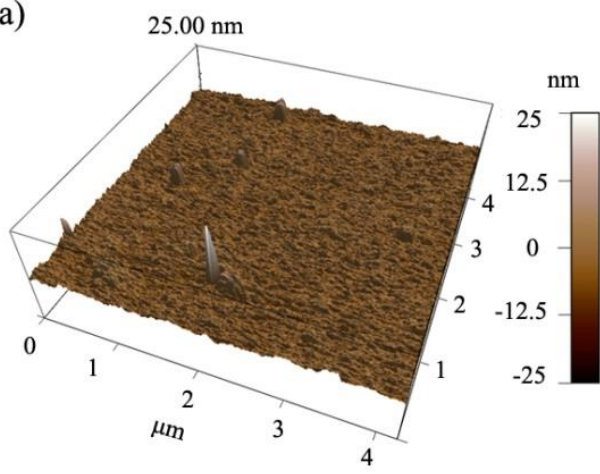

b)

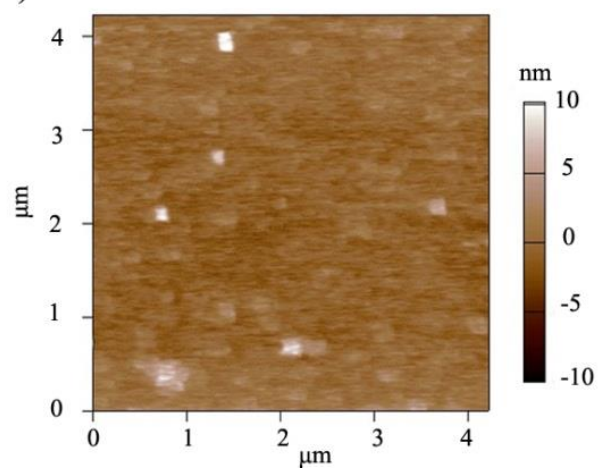

Figure S4: (a) Representative 3D morphology of APTES-glutaraldehyde-anti-kinesin antibodies functionalized surface. (b) Representative 2D morphology of the APTES-glutaraldehyde-anti-kinesin antibodies functionalized surface.

\section{Possible orientations of the APTES on the glass slide}

According to previous research ${ }^{2}$, APTES molecules could potentially assume five different orientations upon immobilization onto glass slides. These are herein defined as "single siloxane", "double siloxane", "triple siloxane", "polymer" and "hydrogen bond", as resulting from the groups presumed to drive such interactions. Each orientation shows different height that could lead to the observed height variation recorded using AFM analysis.

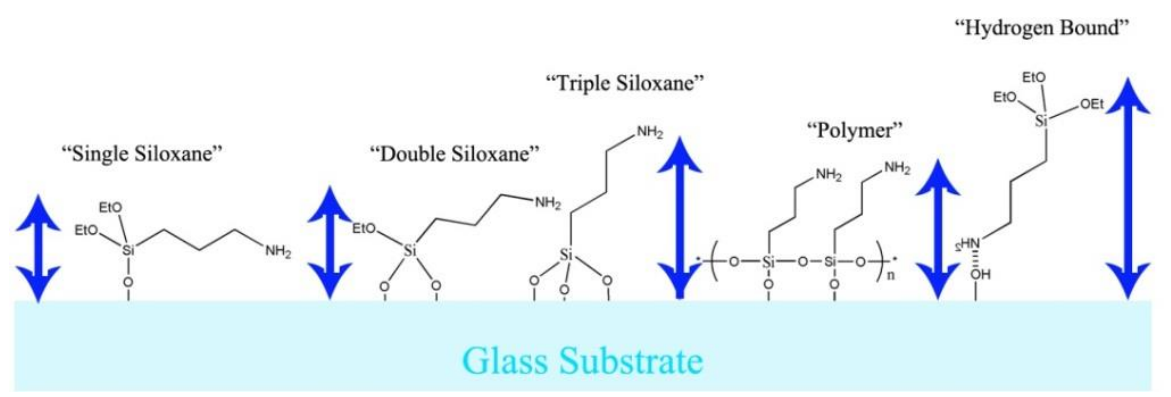

Figure S5: Possible orientations of the APTES at the glass substrate interface.

\section{AFM morphology analyses of the APTES-glutaraldehyde-anti-kinesin antibodies-kinesin functionalized surface}

The morphology of the APTES-glutaraldehyde-anti-kinesin antibodies functionalized surface was evaluated according to the protocol described above. Herein the kinesin had to be printed using the protocols enlisted in the main paper. $R_{q}$ and $R_{a}$ of the APTES-glutaraldehyde-anti-kinesin antibodies-kinesin functionalized substrate were calculated and equaled 4.00 and $5.02 \mathrm{~nm}$ respectively. 
a)

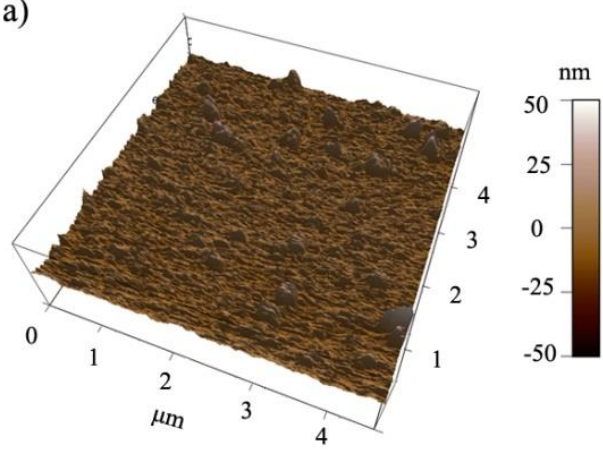

b)

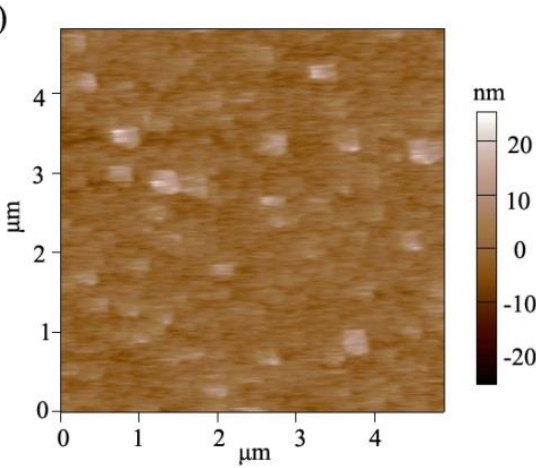

Figure S6: (a) Representative 3D AFM morphology of the APTES-glutaraldehyde-anti-kinesin antibodies-kinesin functionalized surface. (b) Representative 2D AFM morphology of the APTES-glutaraldehyde-anti-kinesin antibodies-kinesin functionalized surface. 


\section{References:}

1 Gadelmawla, E. S., Koura, M. M., Maksoud, T. M. A., Elewa, I. M. \& Soliman, H. H. Roughness parameters. Journal of Materials Processing Technology 123, 133-145, (2002).

2 Acres, R. G. et al. Molecular structure of 3-aminopropyltriethoxysilane layers formed on silanol-terminated silicon surfaces. The Journal of Physical Chemistry C 116, 6289-6297 (2012).

$3 \mathrm{Hu}, \mathrm{X}$., Dong, C., Su, R., Xu, Q. \& Dinu, C. Z. Protein self-assembly onto nanodots leads to formation of conductive bio-based hybrids. Scientific reports 6, 38252 (2016).

4 Dinu, C. Z. et al. Parallel Manipulation of Bifunctional DNA Molecules on Structured Surfaces Using Kinesin - Driven Microtubules. Small 2, 1090-1098 (2006). 
Chapter 3: Protein self-assembly onto nanodots leads to formation of conductive bio-based hybrids

\begin{abstract}
The next generation of nanowires that could advance the integration of functional nanosystems into synthetic applications from photocatalysis to optical devices need to demonstrate increased ability to promote electron transfer at their interfaces while ensuring optimum quantum confinement. Herein we used the biological recognition and the self-assembly properties of tubulin, a protein involved in building the filaments of cellular microtubules, to create stable, free standing and conductive sulfur-doped carbon nanodots-based conductive bio-hybrids. The physical and chemical properties (e.g., composition, morphology, diameter etc.) of such user-synthesized hybrids were investigated using atomic and spectroscopic techniques, while the electron transfer rate was estimated using peak currents formed during voltammetry scanning. Our results demonstrate the ability to create individually hybrid nanowires capable to reduce energy losses; such hybrids could be used in the future for the advancement and implementation into nanometer-scale functional devices.
\end{abstract}




\section{Introduction}

Nanowires have numerous interesting properties such as direct band gaps resulted from their quantum confinement ${ }^{1}$, or precise control of their composition ${ }^{2}$. Geometrical anisotrophy, increased surface to volume ratios, and dipolar magnetic properties are function of the nanowires shape ${ }^{3}$ and were shown to influence their ability to transfer energy at interfaces, mostly because of the confinement of the electrons or the energy levels these electrons occupy ${ }^{4}$. For instance, Fuhrmann et al. ${ }^{5}$ reported a lithography method combined with molecular beam epitaxy, while Choi et al. ${ }^{6}$ used an interface lithography technology combined with catalytic etching to create silicon nanowires arrays with demonstrated interfacial electron transfer. In addition, chemical vapor deposition (CVD) was employed to fabricate germanium-based nanowires at $275^{\circ} \mathrm{C}^{7}$. However, such methods only allow for a limited control of the nanowire's structure-property relationships thus limiting its implementation in nano-functional devices. Further, the growth in porous templates typically produces polycrystalline materials of large diameters thus not offering the small size necessary to generate the quantum confinement effect ${ }^{8}$. Moreover, the precursors used in the CVD process for instance, may be highly toxic (nickel tetracarbonyl), explosive (diborane), or corrosive (silicon tetrachloride) ${ }^{9}$, thus posing a logistical burden to both the environment and the user. Lastly, the tedious and time consuming steps that the lithography technology requires restrict the large scale synthesis of such nanowires ${ }^{10}$.

Combining atoms or individual nanostructures might hold the promise for the next generation of alternative strategies aimed to create bottom-up nanowires to be integrated in synthetic nanodevices. As such, carbon nanodots (C-dot), i.e., photo-stable nanomaterials made out of carbon ${ }^{11}$, have generated considerable interest for nanowires formation due to their ability to photo-induce electron transfer at their interfaces ${ }^{12}$. Claims about effective charge-transfer properties also rendered C-dots usage as viable candidates for nanowire-based hybrid formation that could possibly reduce energy losses while ensuring efficient transport of electrons ${ }^{13}$. However, so far reports only showed C-dots applications in photocatalysis 14,15, storage and transport of electrons ${ }^{16,17}$, lighting systems ${ }^{18,19}$, bioimaging 20,21, nanosensors ${ }^{22,23}$ with improved surface-enhanced Raman scattering ${ }^{24,25}$ or single-particle spectroscopy with photoluminescence ability varying with C-dot sizes ${ }^{15}$. For instance Kang et al. ${ }^{26}$ have demonstrated that iron oxide $\left(\mathrm{Fe}_{2} \mathrm{O}_{3}\right)$-C-dot-based composites have a higher photocatalytic activity as well as continuous and broad absorption in the 550-800 $\mathrm{nm}$ range relative to bare $\mathrm{Fe}_{2} \mathrm{O}_{3}$ nanoparticles. Bao et al. ${ }^{27}$ found that the red shift in C-dot emission 
capability was independent of its size. Properties such as strong photoluminescence resulted from the recombination of photo-generated charges on the defect centers of the C-dot ${ }^{28}$ were exploited for the creation of the next generation of fluorescent probes capable to detect mercury in solution ${ }^{29,30}$ or for $\mathrm{Fe}^{3+}$ detection ${ }^{31}$. Complementarily, the high aqueous solubility, nanoscale size which resemble biological agents such as viruses, bacteria or pathogens ${ }^{32}$, as well as their increased biocompatibility ${ }^{33}$ were explored for drug delivery applications ${ }^{34,35}$ or for ultra sensitive detection of tumor markers such as prostate protein antigen ${ }^{36}$. However, before the C-dots integration in functional devices is to be achieved, charge-transfer properties at their interfaces need to be correlated to their ability to form conductive and controllable geometries.

Several strategies attempted to create nanometer-scale integrated circuits to be used for optical devices ${ }^{37}$ by considering closely spaced metallic nanodots as a possible solution to reduce energy losses at interfaces ${ }^{38}$. For instance, Nomura et al. ${ }^{39}$ fabricated a nanodot coupler from closely spaced structures and demonstrated its ability to convert propagating far-field to an optical near-field light ${ }^{40}$. The high efficiency recorded in such an approach was a result of the coupling of the scattering at the metallic nanodot interfaces ${ }^{41}$. Furthermore, when nanodot arrays were used as substrates, localized surface plasmon resonance detection of antigen-antibody binding was also demonstrated, with the analyses showing that the extinction peaks intensities associated with the specific detection were dependent on the height of the nanodot arrays as well as influenced the array's ability to detect the light transmitted at its interface ${ }^{42}$. However, while these examples demonstrate the ability to create individual surface nanodot-based structures, they do not provide the proof-of-concept that the scaffold-addressable nanodots forming such structures are capable to reduce energy losses or advance nanometer-scale implementation of nanowires in functional devices.

We aim to create the next generation of hybrid nanowires through self-recognition and self-assembly properties of biological molecules responsible for cell structure formation ${ }^{43}$. Specifically, using user-synthesized sulfur-doped C-dots templates and tubulin, the precursor of microtubules cytoskeletal filaments, we demonstrated the ability to control the synthesis and stability of bio-based hybrid nanowires as well as their conductivity, to thus help evaluate their forecast for implementation in the next generation of functional nanodevices with nanometer size distribution and high quantum yield. 


\section{Materials and Methods}

\section{Synthesis and characterization of the sulfur-doped carbon nanodots}

Sulfur-doped carbon nanodots (S-doped C-dots) were synthesized using a hydrothermal method ${ }^{31}$. Briefly, $0.1 \mathrm{M} 25 \mathrm{~mL}$ sodium citrate and sodium thiosulfate solutions (Tianjin Guangfu Technology Development Co., Ltd., China) were added into a $50 \mathrm{~mL}$ Teflon-lined stainless steel autoclave (Fuzhou Keleisi Test Equipment Co., Ltd., China) and the autoclave was incubated at $200{ }^{\circ} \mathrm{C}$ for $6 \mathrm{~h}$. The resulting products were filtered through a $0.22 \mu \mathrm{m}$ filtration membrane (Spectrum Laboratories, Inc. USA).

Such user-synthesized S-doped C-dots were further characterized using both morphological and structural-based techniques. Specifically, for sample size and morphology analyses, contact mode Atomic Force Microscopy (AFM, Asylum Research, USA) with a silicon nitride tip (TR-400PB, Asylum Research, USA) in ambient or in solution was used. The trigger force was kept constant at $3 \mathrm{nN}$ while the spring constant of the cantilever was measured before each experiment using established method ${ }^{44}$. For these analyses, mica sheets were first washed with deionized water (DI water), ethanol (90\%, Fisher Scientific, USA), and again with DI water, and subsequently dried under vacuum overnight and at the room temperature. The cleaned mica was subsequently functionalized with 3-aminopropyltriethoxysilane (APTES, Fisher Scientific, USA; $20 \mu \mathrm{L}$, 99\%) through incubation at room temperature for $15 \mathrm{~min}$; any remaining solution was removed under vacuum. Solution of C-dots in DI water $(20 \mu \mathrm{L} ; 0.05 \mathrm{mg} / \mathrm{mL})$ was dropped onto the APTES functionalized mica and either dried under vacuum at room temperature for $3 \mathrm{~h}$ or used directly for imaging in contact mode.

Elemental composition of the user-synthesized S-doped C-dots was evaluated using Energy Dispersive X-ray (EDX, JEOL JSM-7600F Scanning Electron Microscope, USA) and X-ray Photoelectron (XPS, Physical Electronics PHI 5000 VersaProbe XPS/UPS, USA) spectroscopies. The EDX was operated at $20 \mathrm{kV}$ while the pass energy for XPS was maintained at $117.4 \mathrm{eV}$ with a $0.5 \mathrm{eV}$ step for survey scan or $23.5 \mathrm{eV}$ and $0.05 \mathrm{eV}$ for detailed scan respectively.

Attenuated Total Reflectance Fourier Transform Infra-Red Spectrometry (ATR-FTIR; Digilab FTS 7000/UMA 600, USA) allowed for specific functional groups associated with the user-synthesized S-doped C-dots to be identified. The FTIR scans where performed in the 700 to $4000 \mathrm{~cm}^{-1}$ range using a scan speed of $20 \mathrm{kHz}$ and a 10 reflection diamond ATR. The FTIR software packages incorporated an ART correction algorithm for the resulted spectra.

\section{Synthesis of microtubule hybrid bio-nanowires or S-doped C-dots hybridized}




\section{microtubule}

Microtubules were synthesized according to established protocol ${ }^{45}$ from free tubulin incubated in a microtubule polymerization solution. Briefly, the microtubule polymerization solution was obtained by vortexing $5 \mu \mathrm{L} 100 \mathrm{mM}$ magnesium chloride $\left(\mathrm{MgCl}_{2}\right.$, Fisher Scientific, USA), with $6 \mu \mathrm{L}$ dimethyl sulfoxide (DMSO, 99.7\%, Fisher Scientific, USA), 5 $\mu \mathrm{L} 25 \mathrm{mM}$ guanosine-5'-triphosphate (GTP, Sigma, USA) and $9 \mu \mathrm{L}$ BRB80 buffer (formed from a mixture of $80 \mathrm{mM}$ piperazine-N,N'-bis(2-ethanesulfonic acid buffer, $1 \mathrm{mM} \mathrm{MgCl}$ and $1 \mathrm{mM}$ ethylene glycol tetraacetic acid (EGTA), $\mathrm{pH}$ 6.8; all reagents were purchased from Fisher Scientific, USA).

To initiate microtubule polymerization, $2.5 \mu \mathrm{L}$ polymerization solution was injected into $10 \mu \mathrm{L}$ of $4 \mathrm{mg} / \mathrm{mL}$ biotin-tubulin (Cytoskeleton Inc, USA) and the mixture was incubated at $37^{\circ} \mathrm{C}$ for $30 \mathrm{~min}$. To stabilize the resulting microtubules, the solution was dispersed in $1 \mathrm{~mL}$ BRB80 buffer containing $10 \mu \mathrm{M}$ taxol (Fisher Scientific, USA). The stabilized microtubules were kept at room temperature for experimental usage.

For the bio-hybrid synthesis also called hybrid microtubule, first biotin-tubulin-S-doped C-dots-conjugates were formed using non-specific binding of biotin-tubulin onto S-doped C-dots scaffolds ${ }^{46}$. Briefly, $1 \mu \mathrm{L} 6 \mathrm{mg} / \mathrm{mL}$ synthesized S-doped C-dots were injected into a $600 \mu \mathrm{L}$ eppendorf tube containing $5 \mu \mathrm{L}$ of $4 \mathrm{mg} / \mathrm{mL}$ biotin-tubulin and the mixture was incubated for $2 \mathrm{~h}$ at $200 \mathrm{rpm}$ in an ice bath. Hybrids were assembled from the conjugates and free biotin-tubulin in solution as previously described. Briefly, $5 \mu \mathrm{L}$ of $4 \mathrm{mg} / \mathrm{mL}$ free biotin-tubulin was mixed with the biotin functionalized tubulin- S-doped C-dots conjugates and an additional $2.5 \mu \mathrm{L}$ microtubule polymerization solution, and subjected to $37{ }^{\circ} \mathrm{C}$ for 30 min. When time elapsed, the hybrids were stabilized in BRB80 buffer containing $10 \mu \mathrm{M}$ taxol and used for the experiments.

\section{Biological-based sample AFM analyses}

The biological-based samples (either microtubules, S-doped C-dots hybridized microtubules or biotin-tubulin) were analyzed for their morphology and physical characteristics using the AFM previously introduced (see quantum dots section). For this, 20 $\mu \mathrm{L}$ of the sample with or without $0.5 \%$ glutaraldehyde (Fisher Scientific, USA) was dropped onto the APTMS functionalized mica surface (see protocol above) and incubated for $1 \mathrm{~h}$ at room temperature. After the incubation, the surface was washed with $40 \mu \mathrm{L}$ BRB80 buffer containing $10 \mu \mathrm{M}$ taxol for at least 3 times. 
The AFM images were collected immediately after the washing step using contact mode AFM in solution as previously described (see quantum dots section). For these measurements the cantilever's spring constant was calibrated using the thermal noise method ${ }^{44}$; the scan rate of the tip was fixed at $0.5 \mathrm{~Hz}$ for all the experiments. At least 50 (20 for microtubule, 24 for S-doped C-dots hybridized microtubules or 6 biotin-tubulin) biological-based samples have been investigated to collect their morphology and for diameter analyses. The height of the samples (i.e., S-dots, microtubules, or hybrids) was estimated by running linear cross-sections at different points.

For the microtubule diameter, we analyzed at least 72 individual microtubules collected from 20 independent images. The diameter distribution of S-doped C-dots hybridized microtubules was collected from 24 independent AFM images that included 93 individual microtubules. Lastly, no deconvolution of the tip was performed since previous analyses have showed that the width of the small objects as collected using AFM is often distorted due to the combination of the tip and sample geometries, while the height is not ${ }^{47,48}$.

\section{Fabrication of the modified gold electrodes}

To prepare for voltammetry measurements, 4 gold electrodes (CH Instrument Inc., USA) were first cleaned by immersion in Piranha solution (containing 96.4\% sulfuric acid, $\mathrm{H}_{2} \mathrm{SO}_{4}$, Fisher Scientific, USA) and 30\% hydrogen peroxide $\left(\mathrm{H}_{2} \mathrm{O}_{2}\right.$, Fisher Scientific, USA) in a 3:1 $(\mathrm{v}: \mathrm{v})$ for $10 \mathrm{~min}$, then rinsed thoroughly with the DI water ${ }^{49}$. Subsequently, the gold electrodes were polished successively with $1.0,0.3$ and $0.05 \mu \mathrm{m} \alpha$-alumina $(\mathrm{CH}$ Instrument Inc., USA) powders and again rinsed with DI water to remove any impurities resulted from such polishing.

All the clean gold electrodes were further activated by applying electrochemical oxidation-reduction cycles in sulfuric acid ${ }^{50}$; specifically, the potential was cycled from -200 to $1600 \mathrm{mV}$ (vs. $\mathrm{Ag} / \mathrm{AgCl}$ ) at a scan rate of $50 \mathrm{mV} / \mathrm{s}$ in $0.5 \mathrm{M}$ sulfuric acid on a VersaSTAT 3 potentiostat/galvonostat (Princeton Applied Research, USA). The process was repeated until the cyclic voltammetry graph read for each of the electrodes became stable. Upon the treatment, the electrodes were rinsed thoroughly with the DI water and then used for sample functionalization analyses. Briefly, for the chitosan functionalization, $10 \mu \mathrm{L} 1 \%$ (wt) chitosan in BRB80 containing $10 \mu \mathrm{M}$ taxol was incubated onto the surface of one of one electrode, with the incubation being performed under vacuum and overnight. For the S-doped C-dots, microtubules or S-doped C-dots hybridized microtubules functionalization of the remaining 3 
electrodes respectively, $10 \mu \mathrm{L} \mathrm{1 \%}$ (wt) of chitosan in BRB80 containing $10 \mu \mathrm{M}$ taxol was mixed with either $10 \mu \mathrm{L}$ S-doped C-dots $(0.5455 \mathrm{mg} / \mathrm{mL})$, microtubules $(32 \mathrm{nM})$ or S-doped C-dots hybridized microtubules $(32 \mathrm{nM})$, and subsequently the solution was dropped onto each of the activated/clean electrodes and incubated overnight, all under vacuum.

\section{Electrochemical measurement}

Differential pulse voltammetry (DPV) and electrochemical impedance spectroscopy (EIS) were performed on the VersaSTAT 3 potentiostat/galvonostat. For the DPV, the voltage ranged from $0.2 \mathrm{~V}$ to $-0.6 \mathrm{~V}$ with a $50 \mathrm{~ms}$ pulse period, $49 \mathrm{~ms}$ pulse width, $50 \mathrm{mV}$ pulse height and $1 \mathrm{mV}$ pulse potential increment respectively. The DPV analyses were performed using $1.5 \times 10^{-4} \mathrm{M}$ methylene blue (MB, Fisher Scientific, USA) in BRB80 buffer containing10 $\mu \mathrm{M}$ taxol and $10 \mathrm{mM}$ sodium chloride (NaCl, Fisher Scientific, USA). For the EIS, the frequency ranged from $0.01 \mathrm{~Hz}$ to $100000 \mathrm{~Hz}$, while the amplitude of $5 \mathrm{mV}$ was maintained constant. The EIS analyses have been performed in $50 \mathrm{mM}$ potassium ferricyanide $\left(\mathrm{K}_{3} \mathrm{Fe}(\mathrm{CN})_{6}\right.$; Fisher Scientific, USA) in BRB80 buffer containing $10 \mu \mathrm{M}$ taxol. The choice of feerricyanide was based on previous literature reports which showed that this agent is suitable for evaluating electron transfer at an electrode surface ${ }^{51,52}$. The supporting electrolyte used in our experiments is $10 \mathrm{mM} \mathrm{NaCl}$. At least 6 experiments have been performed for each of the samples being reported. 


\section{Results and Discussion}

Herein we hypothesized that hybrid nanowires containing carbon nanodots (C-dots) can be obtained by using self-assembly and self-recognition-based biological processes. Further, we hypothesized that such hybrids' characteristics (both physical and chemical) can be manipulated to increase hybrid' operational stability and thus extend its features for possible nanometer-based device implementation.

To demonstrate our first hypothesis, we synthesized nanowire-based templates of sulfur-doped carbon nanodots (S-doped C-dots) using a hydrothermal method and a mixture of sodium citrate and sodium thiosulfate solutions. Previous analyses showed that such S-doped C-dots have a high fluorescence quantum yield (67\%) and could be used as fluorescent probes for iron detection ${ }^{31}$. User-synthesized S-doped C-dots were evaluated for their physicochemical properties using atomic force microscopy (AFM), Fourier Transform Infrared Spectroscopy (FTIR), Energy-dispersive X-ray (EDX) and X-ray photoelectron (XPS) spectroscopy.

AFM analyses performed in visual fields of $4 \mu \mathrm{m} \times 4 \mu \mathrm{m}$ allowed for both $\mathrm{S}$-doped C-dots as well as any resulting conglomerates identification. Well-separated and quasi-spherical nanodots are shown in Figure 1a insert, with the diameter distribution being shown in Figure 1b. Analyses revealed S-doped C-dots of a rather narrow size (between 2 to $10 \mathrm{~nm}$ ) and an average of about $7.3 \mathrm{~nm}$ (about 620 nanodots were analyzed). More than $81 \%$ of the nanodots showed diameters of less than $10 \mathrm{~nm}$ indicating controlled synthesis that allowed for tight size selectivity. AFM analyses of S-doped C-dots sizes were also confirmed using high resolution transmission electron microscopy (HRTEM- Supplementary Information, Figure S1).

FTIR help evaluate the chemistry of the user-synthesized S-doped C-dots to further ensure the feasibility of the method used for lab synthesis. Figure 1c identifies a broad peak at $3333 \mathrm{~cm}^{-1}$ which was attributed to the stretching vibration of the $\mathrm{O}-\mathrm{H}$ bonds in the carboxylic acid $(-\mathrm{COOH})$ group and/or intercalated water molecules obtained during the S-doped C-dots synthesis ${ }^{53}$. The peak at around $2338 \mathrm{~cm}^{-1}$ was associated with the carbon dioxide $\left(\mathrm{CO}_{2}\right)$ vibration ${ }^{54}$ which could presumably be due to the mismatch between the $\mathrm{CO}_{2}$ concentration in the atmosphere during the baseline acquisition and the atmospheric $\mathrm{CO}_{2}$ concentration during the measurements, while the peak at $1582 \mathrm{~cm}^{-1}$ represents the fingerprint region of the carbonyl group $(\mathrm{C}=\mathrm{O})$, carbon oxygen bond $(\mathrm{C}-\mathrm{O})$ and hydroxyl $(-\mathrm{OH})$ groups ${ }^{55}$. The additional peak observed at $1423 \mathrm{~cm}^{-1}$ was ascribed to the $-\mathrm{COOH}$ 
group ${ }^{56}$ while the peak at $1113 \mathrm{~cm}^{-1}$ was attributed to the $\mathrm{C}-\mathrm{O}$ stretching and the association of the $-\mathrm{OH}$ bonds ${ }^{57}$. Lastly, the peak at $993 \mathrm{~cm}^{-1}$ was attributed to $\mathrm{C}-\mathrm{O}$ stretching and deformation ${ }^{57}, \mathrm{C}-\mathrm{O}-\mathrm{C}$ bonds or free sulfite $\left(\mathrm{SO}^{3-}\right)^{58}$ respectively.

EDX (Figure 1d) and XPS (Figure 1e) analyses helped evaluate the elemental composition of the user-synthesized S-doped C-dots and confirmed the presence of the starting elements used in such synthesis, namely $\mathrm{C}, \mathrm{O}, \mathrm{Na}$ and $\mathrm{S}$. A detailed component analysis of their \% distribution is shown in Table 1. High resolution XPS (Figure 1f and 1g) complemented the FTIR spectrum and confirmed the presence of sulfur $\left(\mathrm{S}_{2 \mathrm{p}}\right)$, with two peaks, one at around $162.11 \mathrm{eV}$ and the second one at $163.29 \mathrm{eV}$. The peaks were attributed to the sulfion $\left(\mathrm{S}^{2-}\right)^{59}$ and sulfite ion $\left(\mathrm{SO}_{3}{ }^{2-}\right){ }^{60}$ respectively, as resulted from the synthesis process. Complementarily, the $\mathrm{C} 1 \mathrm{~s}$ spectra is shown in Figure $\mathbf{1 g}$ and reveals characteristics associated with the $\mathrm{C}-\mathrm{C}(284.7 \mathrm{eV}), \mathrm{C}-\mathrm{H}(284.7 \mathrm{eV}){ }^{61}, \mathrm{C}-\mathrm{O}(285.87 \mathrm{eV}){ }^{62}$ and $\mathrm{C}=\mathrm{O}(288.43$ eV) ${ }^{63}$ respectively.

Table 1. Percentage distribution of the elements present in the user-synthesized S-doped C-dots.

\begin{tabular}{|c|c|c|c|c|c|}
\hline Element & $\begin{array}{c}\text { Carbon } \\
(\mathbf{C})\end{array}$ & $\begin{array}{c}\text { Oxygen } \\
(\mathbf{O})\end{array}$ & $\begin{array}{c}\text { Sodium } \\
(\mathbf{N a})\end{array}$ & $\begin{array}{c}\text { Sulfur } \\
(\mathbf{S})\end{array}$ & $\begin{array}{c}\text { Phosphor } \\
(\mathbf{P})\end{array}$ \\
\hline EDX & 22.77 & 22.06 & 21.50 & 33.67 & 0 \\
\hline XPS & 34.81 & 39.81 & 16.32 & 7.73 & 1.33 \\
\hline
\end{tabular}



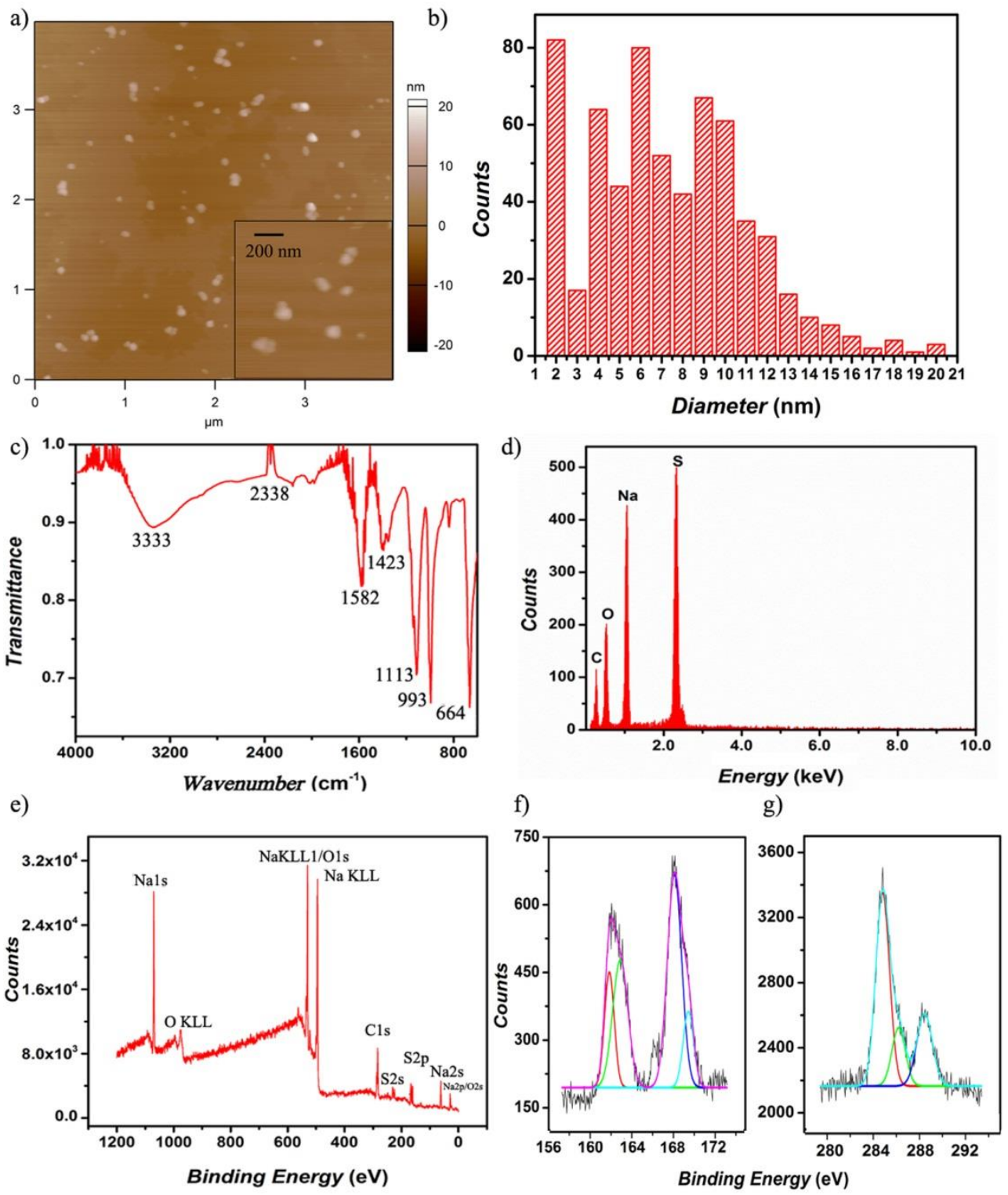

Figure 1: Surface characterization of the S-doped C-dots. a) Representative Atomic Force Microscopy (AFM) analyses that allow for individual or conglomerates of nanodots visualization. AFM insert in the scale of $1 \mu \mathrm{m} \times 1 \mu \mathrm{m}$ allows for particle size analyses. b) The diameter distribution of the S-doped C-dots as resulted from AFM evaluations. c) Fourier Transform Infrared Spectroscopy (FTIR) of the S-doped C-dots allows for identification of surface functional groups, while Energy Dispersive X-Ray (EDX) analysis (d) reveals the elemental composition of the S-doped C-dots. e) The X-ray Photoelectron Spectroscopy (XPS) survey graph of the S-doped C-dots. f) High-resolution $\mathrm{S}_{2 \mathrm{p}}$ XPS spectra of the S-doped C-dots. g) High-resolution $\mathrm{C}_{1 \mathrm{~s}}$ XPS spectra of the S-doped C-dots. 
Secondly, we evaluated the electrochemical properties of the user-synthesized and above characterized S-doped C-dots using differential pulse voltammetry (DPV; Figure 2). For this, chitosan, a linear polysaccharide was used to suspend the S-doped C-dots to form a membrane onto the DPV electrodes; the electrochemical reaction efficiency was based on the rate of ion generation upon interaction with methylene blue (MB) (Figure 2a). The organic dye MB was chosen based on previous analysis that showed the dye to be an efficient electrochemical agent capable to allow for changes in intensity as resulted from the reduction and oxidation induced currents to be quantified ${ }^{64}$. Previous studies have also showed that charge-transfer properties are observed when using electron-donating C-dots and electron-accepting perylenediimides ${ }^{65}$; studies demonstrated that $\mathrm{C}$-dots can be integrated in nafion nanocomposite to enhance the detection sensitivity of an electrochemical immunosensor ${ }^{66}$. Further, analyses revealed that chitosan is a suitable carrier material for electrochemical biosensors ${ }^{67}$, with Sun et al. ${ }^{68}$ using chitosan containing embedded acetylcholinesterase to probe kinetics at interfaces. Complementarily, Li et al. ${ }^{69}$ developed a chitosan-embedded nonenzymatic glucose sensor that was based on glassy carbon electrode (GCE) coated with platinum hollow nanoparticle chains dispersed onto porous gold nanoparticles. Lastly, $\mathrm{Xu}$ et al. ${ }^{70}$ entrapped hemoglobin into a graphene and chitosan composite film for direct electrochemical behavior on a GCE.

Compared with the chitosan only, the user-synthesized S-doped C-dots embedded in the chitosan membrane provided a conductive microenvironment that facilitated electron transfer as shown by the increase of the peak current of this membrane relative to the only chitosan-based membrane (Figure 2b). Specifically, peaks centered at about $-0.22 \mathrm{~V}$ were observed for both membranes (with and without the embedded S-doped C-dots respectively; Figure 2c). Additionally, a new peak was observed for the S-doped C-dots embedded in the chitosan membrane, with this peak being centered at about $-0.36 \mathrm{~V}$ (about $-0.58 \mathrm{~V}$ vs. standard hydrogen electrode) and attributed to the redox potential of the sulfite $\left(\mathrm{SO}_{3}{ }^{2-}\right)^{71}$. The relative low intensity of this peak when compared with the one centered at $-0.22 \mathrm{~V}$ was presumably due to the low concentration of the $\mathrm{SO}_{3}{ }^{2-}$ resulted from the S-doped C-dot synthesis as confirmed by the spectroscopy analyses included above.

The electron transfer rate at the S-doped C-dots embedded in the chitosan membrane was estimated using the Laviron's equation ${ }^{72,73}$ (Eq: 1) and compared with the transfer rate at the chitosan membrane only. Specifically, 


$$
E_{p, c}=E^{0^{\prime}}+\frac{R T}{\alpha n F} \ln \frac{R T k_{s}}{\alpha n F}-\frac{R T}{\alpha n F} \ln v
$$

with the cathodic peak potential $\left(\mathrm{E}_{\mathrm{p}, \mathrm{c}}\right)$ being function of the formal potential $\left(\mathrm{E}_{0}\right), \alpha$ the cathodic electron transfer coefficient, the scan rate(v) with the symbols $\mathrm{R}, \mathrm{T}$ and $\mathrm{F}$ representing the ideal gas constant, absolute temperature and Faraday constant respectively, and $\mathrm{n}$ representing the number of electrons involved in the redox reaction.

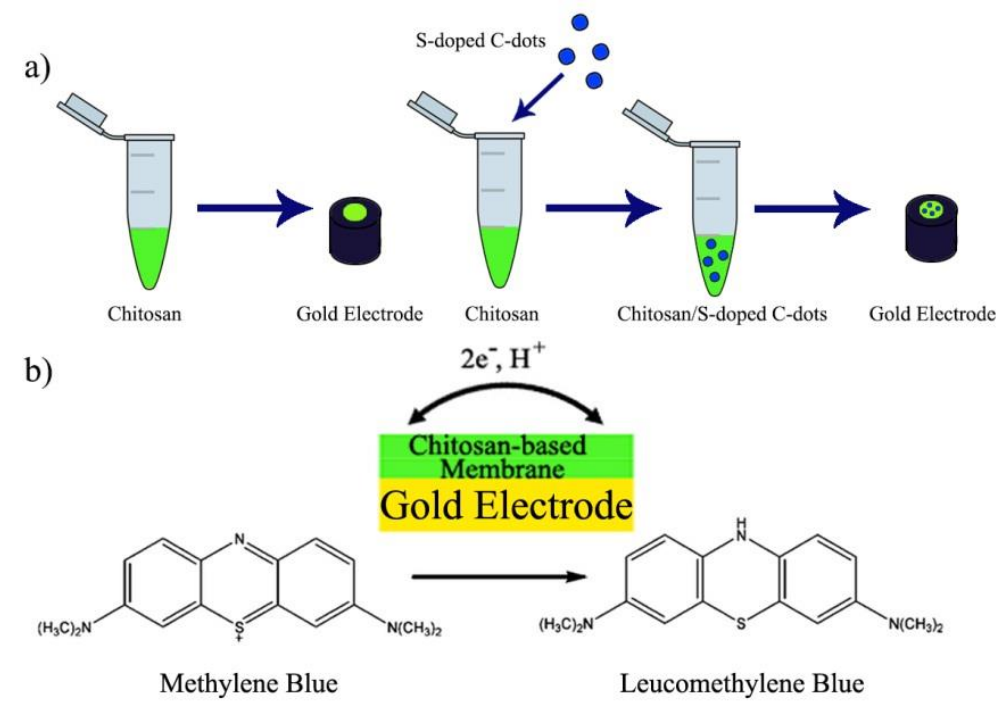

c)

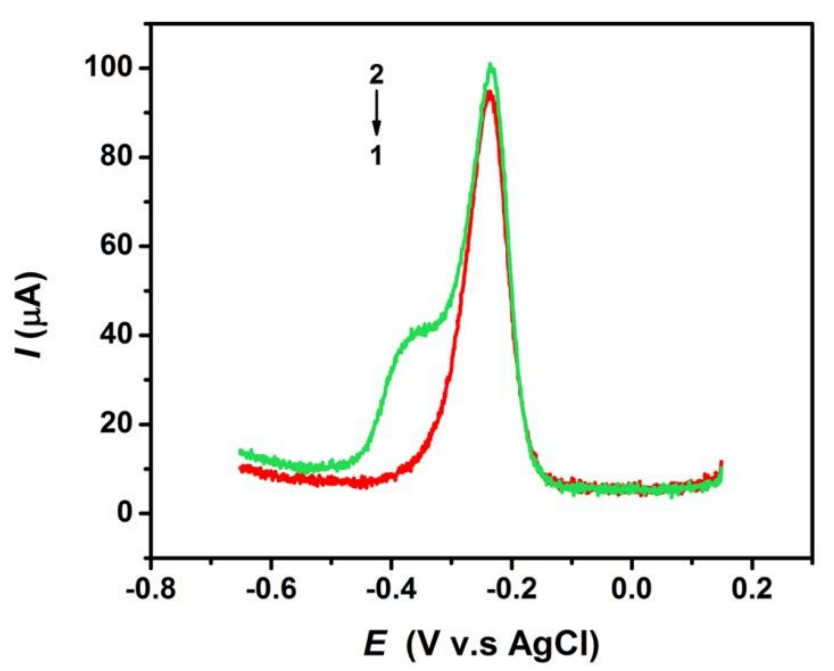

Figure 2: a) Representative schematic of the chitosan-based membrane formation at the gold electrode interface. b) The reduction reaction of the methylene blue (MB) onto the electrode surface. c) Differential Pulse Voltammetry (DPV) graph of the MB on 1) chitosan/gold electrode (red), 2) S-doped C-dots/chitosan/gold electrode (green). 
Analyses showed that the electron transfer coefficient was increased from 3.309 to 4.618 for the S-doped C-dots-containing membrane when compared to the chitosane only membrane, thus indicating a larger electron transfer at the C-dots-containing membrane interface.

Thirdly, we demonstrated the ability to fabricate hybrid bio-nanowires containing S-doped C-dots by using self-assembly and self-recognition of polymeric biomolecules, i.e. tubulin into a two-step strategy. Tubulin is a globular protein that assembly to form a microtubule cytoskeletal structure in the cell, which serves as filament for cell-based transport or for template of cell division ${ }^{74}$. Previous reports have showed that tubulin could be templated onto carbon nanotubes to form hybrid systems with tubular shape ${ }^{75}$ that could alter cell mechanics ${ }^{76}$ and induce toxicity ${ }^{77}$.

In our first step, we formed tubulin-S-doped C-dots conjugates by physical adsorption of free tubulin onto the user-characterized S-doped C-dots ${ }^{46}$. Our theoretical analyses based on the amount of protein used for loading (see materials and methods) and the size characteristics of the user-synthesized S-doped C-dots showed that the protein was adsorbed onto the nanodots to a loading of about maximum 4 tubulins per individual S-doped C-dot. In the second step, the templated tubulin was combined with additional free tubulin to form hybrid bio-nanowires or S-doped C-dots-based nanowires. This was achieved through simply exploiting tubulin known ability to polymerize in the presence of guanosine triphosphate into the microtubular structure ${ }^{45}$.

Synthesized S-doped C-dots-based microtubule hybrids were evaluated for their morphology, integrity and stability using (3-aminopropyl)triethoxysilane (APTES) functionalized mica surfaces and AFM analyses in solution, and compared to control microtubules polymerized only from free tubulin. Figure 3a depicts the average diameter of the hybrid microtubules when compared to microtubules used as controls respectively. Analyses showed that microtubules had an average diameter of about $8 \mathrm{~nm}$, while the diameter of the S-doped C-dots hybrid microtubules was significantly higher, i.e., $14 \mathrm{~nm}$ (student's T-test $\mathrm{p}^{*}<0.05$ ). About $30 \%$ (i.e., 72 microtubules) were about $10 \mathrm{~nm}$ while no more than $5 \%$ of them had a diameter larger than $13 \mathrm{~nm}$ (Figure 3b). Figure 3c shows the diameter distribution of the S-doped C-dots hybrid microtubules, ranging from 6 to $31 \mathrm{~nm}$ with about 23\% (out of the 93 S-doped C-dots hybrid microtubules being analyzed) having an average diameter of about $16 \mathrm{~nm}$. 


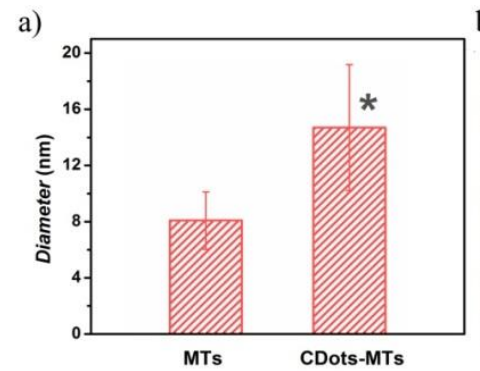

d)

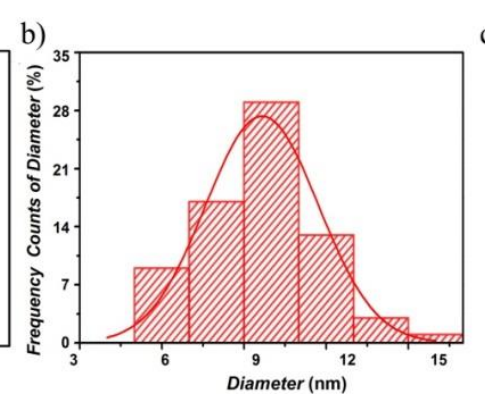

Diameter $(\mathrm{nm})$

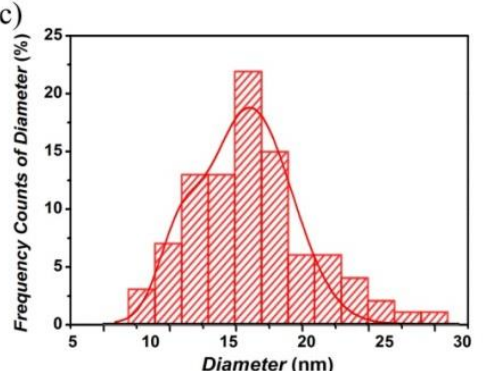

3

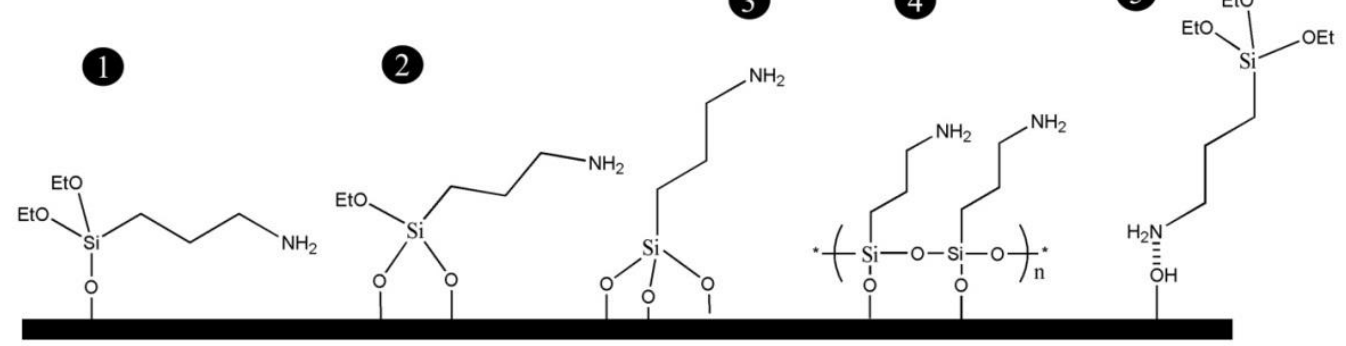

Figure 3: a) Average diameter of microtubules and the S-doped C-dots hybridized microtubules as measured using cross-sectional analyses. b) The diameter distribution of 72 individual microtubules collected from 20 independent images. c) The diameter distribution (as measured using cross-sectional analyses) of S-doped C-dots hybridized microtubules and collected from 24 independent AFM images that included 93 individual microtubules. d) Different orientations or combination of such orientations of the APTES molecules on the mica surface lead to different geometries and size distribution of the microtubules.

The observed uneven diameter distributions are presumably due to the non-uniform absorption of the APTES onto the mica surface as well as from the uneven incorporation of the S-doped C-dots onto the hybrid microtubule structure during the template-tubulin polymerization. The first statement is supported by previous analyses of microtubules in solution ${ }^{78}$ which recorded irregular diameter distributions as result of their irregular absorption onto a similarly functionalized mica. Specifically, with APTES possibly assuming 5 orientations ${ }^{79}$ (Figure 3d) including one (1), two (2) or three (3) adsorbed ethoxy groups, as well as due to the polymerization with multiple ethoxy groups binding to the silanol-terminated silicon (4), or hydrogen bond formation between the $\mathrm{NH}_{2}$ group on the APTES molecule and the mica surface (5), APTES would possibly undergo different orientations thus leading to anisotropic geometries onto the mica surfaces. Such geometries could further lead to different polymer's lengths being exposed above the mica resulting in diverse microtubule embedment and non-uniform distribution of its diameter. Such 
distributions could possibly also be responsible for the variations recorded for the hybridized bio-nanowires, namely the ones containing the S-doped C-dots-based microtubules.

To demonstrate the second hypothesis, i.e., that the characteristics of such user-formed hybrid bio-nanowires can be manipulated to increase their stability, we first performed morphology analyses of both the microtubule (control) and S-doped C-dots hybrid microtubule crosslinked with glutaraldehyde by using AFM contact mode in solution (Figures 4). Glutaraldehyde was chosen to stabilize the microtubule and hybrid structures ${ }^{80}$ since previous studies showed that the compound can be used for both inter and inner molecular condensation of biological molecules ${ }^{81}$, with Walt et al. ${ }^{82}$ showing that glutaraldehyde forms a Schiff's base with the lysine residues in a protein, both under acidic or neutral conditions, and Tashima et al. ${ }^{83}$ respectively showing that an addition reaction occurs in alkaline condition. Further, glutaraldehyde was previously shown to be used as a suitable agent for microtubule crosslinking known to stabilize its gross structure against air drying or a distilled water challenge ${ }^{84}$ with the cross-linked microtubules showing minimal changes from native properties.

Our analyses showed linear-like geometries with non-uniform transversal height distribution for both the microtubule (Figure 4a) and its hybrid bio-nanowire counterpart (Figure 4b) respectively. The morphology of the microtubule was smooth and linear when compared to the entangled and bead-like morphology observed for the hybrid microtubule (Supplementary Information Figure S2). Debris associated with either free tubulin, S-doped C-dots or tubulin-S-doped C-dots conjugates was also observed.

Control experiments performed on microtubules only (i.e., not stabilized with glutaradehyde) showed sample denaturation (Figure 4c) while no denaturation was observed for the counterpart hybrids bio-nanowire (Figure 4d). Specifically, the hybrids did not only kept their morphology under AFM scanning conditions, but further, analyses allowed for their optical examination for up to 9 days which was in contrast with the 5 days recorded for their counterparts. This is presumably due to the amino groups in the tubulin protein reacting with the aldehyde groups in the glutaraldehyde to form chemical bonds ${ }^{85}$ that limited microtubule denaturation and led to its increased stability. Further, the increased stability of the hybrids can also be due to the unsaturated bonds $(\mathrm{C}=\mathrm{O}$ and $\mathrm{C}=\mathrm{C}$ groups $)$ in the $\mathrm{S}$-doped $\mathrm{C}$-dots reacting with the aldehyde groups in glutaraldehyde to limit hybrid denaturation.

Figure $4 \mathbf{e}$ and $\mathbf{4 f}$ show the cross-sectional height distributions (red lines e and $\mathrm{f}$ in Figure $\mathbf{4 a}$ and $\mathbf{4 b}$ respectively) along the glutaraldehyde stabilized microtubule or S-doped 
C-dots hybrid microtubule, respectively. The average height based on cross-sectional analysis of the normal microtubule was about $7 \mathrm{~nm}$ while the height of the $\mathrm{S}$-doped C-dots hybridized microtubule was about $15 \mathrm{~nm}$ with the height distribution of the microtubule seeming more uniform than that of the S-doped C-dots hybrid microtubule.

We also evaluated the electrochemical properties of the S-doped C-dots hybrid microtubule or hybrid bio-nanowires. For this, we first incubated chitosan with the formed hybrids and subsequently used DPV and impedance analyses to assess their conductivity. The consideration for incubating chitosan with the microtubules or hybrids directly is based on previous analyses that showed that interaction of chitosan with proteins forms complexes, mainly through hydrophobic and electrostatic contacts (i.e., function of the protein structural elements ${ }^{86}$ ) however with such interaction inducing protein destabilization. If destabilization would be induced on the tubulin, then no self-assembly or microtubules ${ }^{84}$ formation would be observed; to support this, control experiments in which chitosan was added to only free tubulin were performed (Supplementary Information, Figure S3). The electrodes functionalization with chitosan solution was performed in a manner similar to the one described in Figure 2a. 

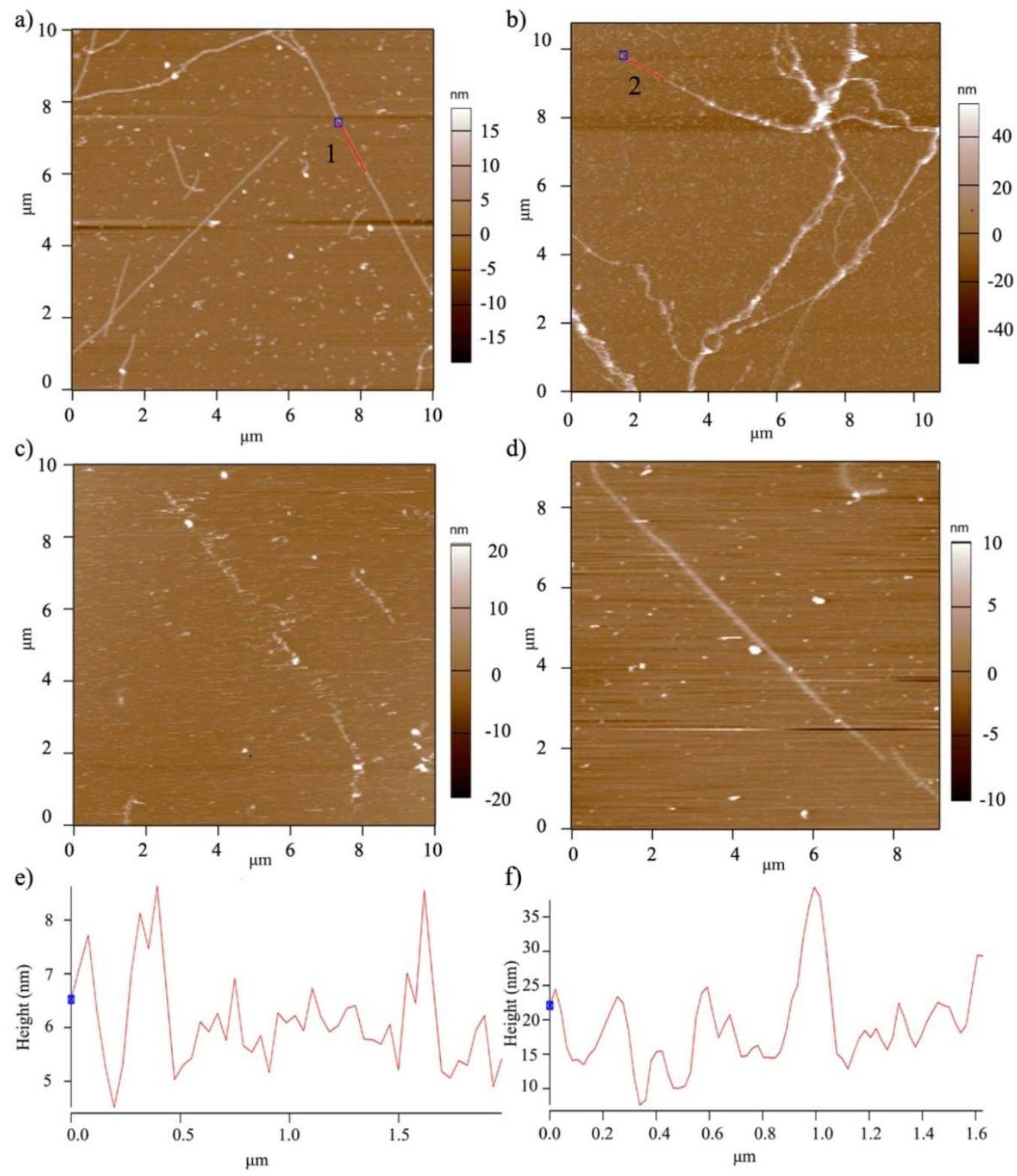

Figure 4: a) Representative morphology AFM analyses of microtubules after crosslinking with glutaraldehyde. b) Representative morphology AFM analyses of S-doped C-dots hybridized microtubules after crosslinking with glutaradehyde. Reduced stability was observed for the microtubules not crosslinking with glutaraldehyde (c) while the morphologies of the hybrid microtubules was not affected by the lack of the crosslinking agent (d). e) Height distribution of the microtubule (along the red line 1 in Figure 4a; cross-sectional analyses) or S-doped C-dots hybridized microtubules (along the red line 2 in Figure $4 \mathbf{b}$; cross-sectional analyses) with the blue square representing the start point $(0.0 \mu \mathrm{m})$. The line followed the sample morphology. 
Figure 5a shows the electrochemical impedance spectrum of a representative bare gold as well as of the functionalized electrode (where functionalization is refereed to the sample of choice), with the impedance recorded in potassium ferrocyanide. Previous investigations showed that potassium ferrocyanide is a suitable agent to help monitor surface functionalization of an electrode with Kafka et al. using the agent to distinguish non-complementary DNA in the electrochemical impedance spectrum ${ }^{87}$ and Moulton et al. to study electron transfer on the gold electrode before and after adsorption of serum albumin ${ }^{88}$.

Generally, the chitosan-based membranes had smaller impedances when they contained embedded S-doped C-dots or hybrids containing the S-doped C-dots thus confirming increased conductivities of such hybrids. Specifically, analyses showed that Nyquist plot ${ }^{89}$, representing the real and imaginary impedances plotted against each other for different perturbation frequencies and known to allow for the charge transfer at the electrode interface to be evaluated, was almost a straight line for the bare electrode (Figure 5a; black curve, i.e., 1). This was attributed to the diffusion limited step of the electrochemical process at this electrode's ${ }^{90}$ and thus indicated a very small value of its measured impedance. However, after chitosan or chitosan-containing embedded components immobilization, the Nyquist plot changed and the electrode impedance increased in a manner dependent with its functionalization. Specifically, the plot showed a larger semicircle corresponding to the larger electron transfer resistance for the S-doped C-dots-chitosan-gold electrode and S-doped C-dots-microtubule-chitosan-gold electrode. Complementarily, the impedance values of the S-doped C-dots-chitosan-gold electrode, microtubule-chitosan-gold electrode and S-doped C-dots-microtubule-chitosan-gold electrode, were about $180 \Omega$ (blue; i.e., 3), $300 \Omega$ (green; i.e., 5) and $240 \Omega$ (pink; i.e., 4) respectively. The lower values of the obtained charge transfer resistances indicated a faster reaction rate at the interfaces with the $\mathrm{C}$-dots nanodots or C-dots-based hybrids respectively, thus confirming the role of the C-dots in enhancing electron transfer at the membrane interfaces with the results being attributed to the fluent electron transfer between the potassium ferrocyanide and such membranes during the electrochemical reaction. Analyses also showed that, when compared with the chitosan-gold electrode, the membrane containing embedded S-doped C-dots in the chitosan membrane immobilized at the gold electrode had an additional peak with its impedance decreasing from $180 \Omega$ to $150 \Omega$ with the peak being a result of the additional species present during the S-doped synthesis and confirmed by the spectroscopy analyses.

The electrochemical behavior of the $\mathrm{MB}$ on the modified electrodes was also 
investigated as described previously. Analyses (Figure 5b) confirmed that the peak current of the MB on the S-doped C-dots hybrid microtubule coated electrode (pink; i.e., 4) was larger than that on the control microtubule-coated electrode (green; i.e., 5) thus indicating that the electrochemical process came from the embedment of the C-dots. The largest peak current (about $105 \mu \mathrm{A}$ ) appeared to be on the bare gold electrode (black; i.e., 1), while the smallest peak current (about $65 \mu \mathrm{A}$ ) was recorded for the microtubule-chitosan-gold electrode (green; i.e., 5). Further, control experiments demonstrated that for hybrids and microtubules can be compared since there is no no-specific dissociation of the quantum dots from the former (Supplementary Information Figure S4).

Our analyses showed that all the curves contained peaks at about $-0.22 \mathrm{~V}$ ( $\mathrm{vs} \mathrm{Ag} / \mathrm{AgCl}$ ) which confirmed the reduction of the $\mathrm{MB}$ cation $\left(\mathrm{MB}^{+}\right)^{91}$, with an observed decrease from the gold, to S-doped C-dots-chitosan-gold electrode, chitosan-gold electrode, S-doped C-dots-microtubule-chitosan-gold electrode, to microtubule-chitosan-gold electrode respectively. In addition, there were two additional peaks at about $-0.38 \mathrm{~V}$ on curve 3 associated with the S-doped C-dot-chitosan-gold electrode and 4 associated to the S-doped C-dot-MTs-chitosan-gold electrode respectively, with such peaks presumably originating from the additional chemicals contained in the S-doped C-dots as demonstrated by the EDX analyses. In particular, previous results have showed that the standard redox potential of $\mathrm{SO}_{3}{ }^{2-}$ is $0.571 \mathrm{~V}$ for instance, while according to the Nernst equation ${ }^{92}$ the redox potential of $\mathrm{SO}_{3}{ }^{2-}$ versus $\mathrm{Ag} / \mathrm{AgCl}$ is $0.38 \mathrm{~V}$ thus being attributed to the reduction reaction of the $\mathrm{SO}_{3}{ }^{2-}$ and/or the reducing of $\mathrm{SO}_{3}{ }^{2-}$ to thiosulfuric $\left(\mathrm{S}_{2} \mathrm{O}_{3}{ }^{2-}\right.$; Eq. 2).

$2 \mathrm{SO}_{3}{ }^{2-}+3 \mathrm{H}_{2} \mathrm{O}+4 \mathrm{e}^{---}-\mathrm{S}_{2} \mathrm{O}_{3}{ }^{2-}+6 \mathrm{OH}^{-} \quad(-0.571 \mathrm{~V})$

It is reasonable that the S-doped C-dots could act as a conducting bridge between the microtubule and the electrode to increase the conductivity of the hybrid structure, thus enhancing the electrochemical intensity effectively and further demonstrating the conductive features of the nanowire being created. 
a)

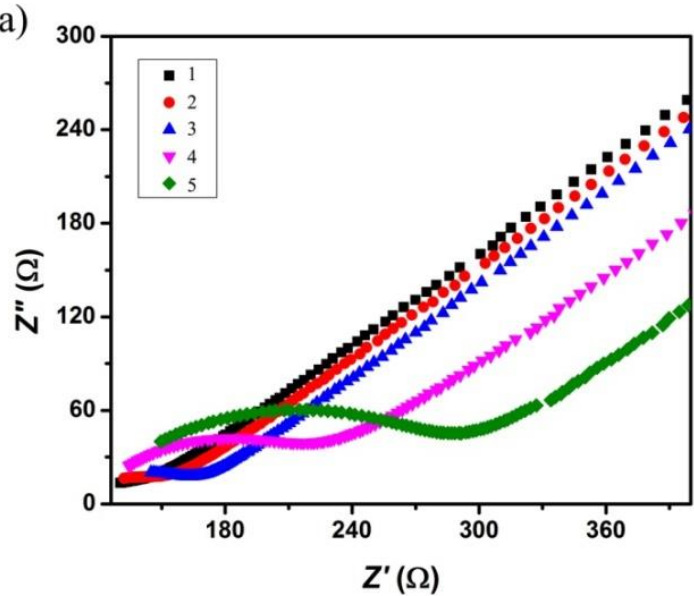

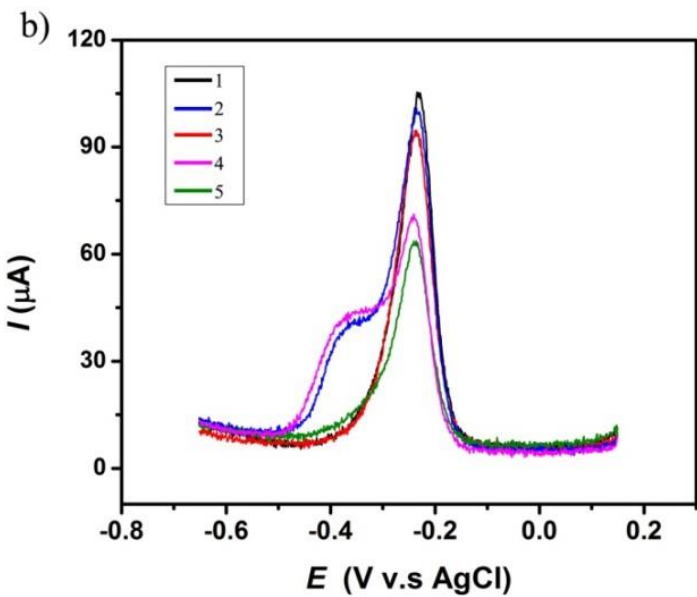

c)
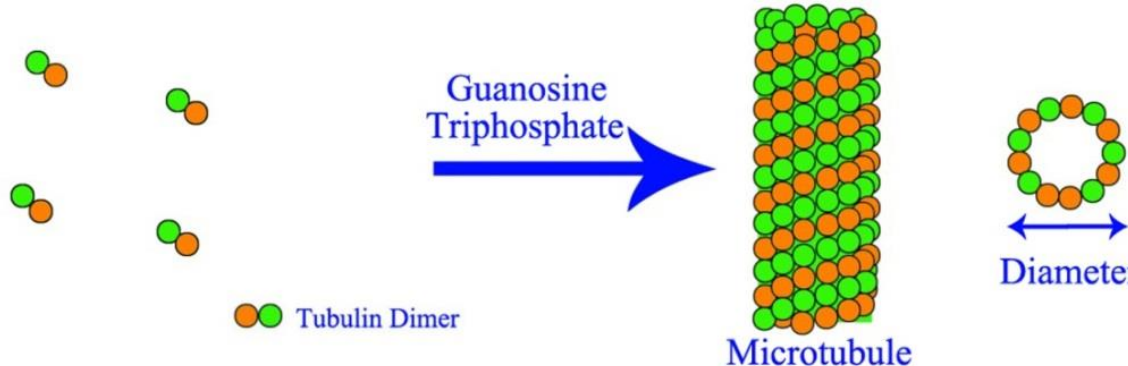

Diameter

Microtubule

d)

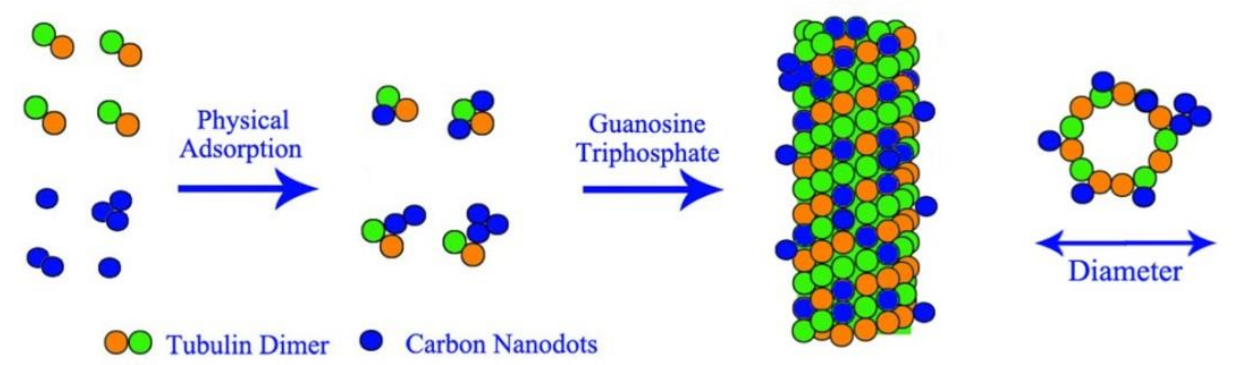

Figure 5: a) Electrochemical impedance spectrum (EIS) graph of the electrode. 1. Bare gold electrode. 2. S-doped C-dots/Chitosan/Gold electrode. 3. Chitosan/Gold electrode. 4. S-doped C-dots-microtubule (MTs)/Chitosan/Gold electrode. 5. Microtubule (MTs)/Chitosan/Gold electrode. b) Differential pulse voltammetry (DPV) graph of the methylene blue on different electrode. The color-coding is similar to what described in a). c) Scheme of microtubule polymerization from free tubulin under the chemical energy of guanosine triphosphate. d) Scheme of the polymerization process of the S-doped C-dots hybrid microtubule; the schematic is not to scale.

Nanowire structures are expected to offer user-controllable surfaces for novel functional hybrids with unique chemical and physical properties. For instance, studies by Xie et al. showed that silicon nanowire/carbon hetero junctions can be fabricated for the next 
generation of photovoltaic devices to show increased power conversion efficiency as resulted from the increased optical absorption at the C-dots interfaces ${ }^{93}$. Complementarily, Zhang et al. synthesized C-dots loaded $\mathrm{TiO}_{2}$ nanorods, with the C-dots serving as green sensitizers and showed that they are capable to enhance the hybrid material photo-responsiveness in visible light ${ }^{94}$.

Herein, the self-organization and self-assembly of tubulin onto thd user-synthesized S-doped C-dots was shown to lead to stable nanowire geometries of increased conductivities and proves to be a feasible alternative to the methods described in this paper's introduction. Specifically, our hybrid microtubule nanowire of only about $14 \mathrm{~nm}$ in diameter and formed in mild conditions could allow for limited inhibition of the quantum confinement effect to thus lead to a functional structure. Further, such S-doped C-dots hybridized microtubule or bio-nanowire could prove to be an easy and "green" step in which new function can be implemented to a non-biological molecule to be recognized and transported to user-defined location by kinesin hangars. In particular, previous studies have showed that microtubule-based transport system, normally used in the cell for long distance transport of cargos, could be mimicked in synthetic environment to allow for transport of nanoparticles or nanotubes ${ }^{43}$. If such a transport can be implemented and observed for the S-doped C-dots hybrid microtubule, then one could envision not only the demonstrated assembly of functional nanowires with increased conductivities but further, their regular positioning under the energy control of adenosine triphosphate used to ensure kinesin biological function. Such approach has the potential to fully achieve the promising applications of such nanodots or resulting nanowires through both their control over the size as well as their orientation onto user-controlled surfaces and geometries ${ }^{43}$. 


\section{Conclusions}

In this study, we successfully synthesized a conductive and hybrid bio-nanowire by using the self-assembly properties of free tubulin (a biological molecule) and tubulin templated onto user-synthesized S-doped C-dots in solution. Our analyses showed that such nanowire is more conductive than control microtubule assembled only from free tubulin. Further, the nanowire had an average diameter of $14 \mathrm{~nm}$ that was superior to any of templated nanowires currently produced. Such synthesized bio-nanowire may increase the signal processing ability for the next generation of integrated circuits while ensuring regular positioning through biological self-recognition capabilities. 


\section{References:}

1 Nolan, M., O'Callaghan, S., Fagas, G., Greer, J. C. \& Frauenheim, T. Silicon nanowire band gap modification. Nano letters 7, 34-38 (2007).

2 Agarwal, R. \& Lieber, C. M. Semiconductor nanowires: optics and optoelectronics. Appl. Phys. A 85, 209-215, doi:10.1007/s00339-006-3720-z (2006).

3 Hossain, M. Z. \& Kleve, M. G. Nickel nanowires induced and reactive oxygen species mediated apoptosis in human pancreatic adenocarcinoma cells. Int $J$ Nanomedicine 6, 1475-1485, doi:10.2147/ijn.s21697 (2011).

4 Xiang, D. Fabrication and utilization of mechanically controllable break junction for bioelectronics. (Forschungszentrum Jülich, 2012).

5 Fuhrmann, B. et al. Ordered Arrays of Silicon Nanowires Produced by Nanosphere Lithography and Molecular Beam Epitaxy. Nano Letters 5, 2524-2527, doi:10.1021/nl051856a (2005).

6 Choi, W. K. et al. Synthesis of Silicon Nanowires and Nanofin Arrays Using Interference Lithography and Catalytic Etching. Nano Letters 8, 3799-3802, doi:10.1021/n1802129f (2008).

7 Wang, D. \& Dai, H. Low - Temperature Synthesis of Single - Crystal Germanium Nanowires by Chemical Vapor Deposition. Angewandte Chemie 114, 4977-4980 (2002).

$8 \mathrm{Hu}$, J., Odom, T. W. \& Lieber, C. M. Chemistry and physics in one dimension: synthesis and properties of nanowires and nanotubes. Accounts of chemical research 32, 435-445 (1999).

9 Creighton, J. \& Ho, P. Introduction to chemical vapor deposition (CVD). Chemical vapor deposition, 2 (2001).

10 Choi, Y.-J. et al. Novel fabrication of an $\mathrm{SnO} 2$ nanowire gas sensor with high sensitivity. Nanotechnology 19, 095508 (2008).

11 Hsu, P.-C., Shih, Z.-Y., Lee, C.-H. \& Chang, H.-T. Synthesis and analytical applications of photoluminescent carbon nanodots. Green Chemistry 14, 917-920 (2012).

12 Zhang, H. et al. Carbon quantum dots/Ag3PO4 complex photocatalysts with enhanced photocatalytic activity and stability under visible light. Journal of Materials Chemistry 22, 10501-10506, doi:10.1039/C2JM30703K (2012). 
13 Strauss, V. et al. Carbon Nanodots: Toward a Comprehensive Understanding of Their Photoluminescence. Journal of the American Chemical Society 136, 17308-17316 (2014).

$14 \mathrm{Li}, \mathrm{H}$. et al. Carbon quantum dots/Cu2O composites with protruding nanostructures and their highly efficient (near) infrared photocatalytic behavior. Journal of Materials Chemistry 22, 17470-17475, doi:10.1039/C2JM32827E (2012).

$15 \mathrm{Li}, \mathrm{H}$. et al. Water-Soluble Fluorescent Carbon Quantum Dots and Photocatalyst Design. Angewandte Chemie International Edition 49, 4430-4434, doi:10.1002/anie.200906154 (2010).

16 Lin, F. et al. Electron transfer quenching by nitroxide radicals of the fluorescence of carbon dots. Journal of Materials Chemistry 22, 11801-11807, doi:10.1039/C2JM31191G (2012).

17 Yan, X., Cui, X., Li, B. \& Li, L.-s. Large, Solution-Processable Graphene Quantum Dots as Light Absorbers for Photovoltaics. Nano Letters 10, 1869-1873, doi:10.1021/nl101060h (2010).

18 Kwon, W. et al. Freestanding Luminescent Films of Nitrogen-Rich Carbon Nanodots toward Large-Scale Phosphor-Based White-Light-Emitting Devices. Chemistry of Materials 25, 1893-1899, doi:10.1021/cm400517g (2013).

19 Wang, C. et al. Upconversion fluorescent carbon nanodots enriched with nitrogen for light harvesting. Journal of Materials Chemistry 22, 15522-15525, doi:10.1039/C2JM30935A (2012).

$20 \mathrm{Xu}, \mathrm{Z}$.-Q. et al. Low temperature synthesis of highly stable phosphate functionalized two color carbon nanodots and their application in cell imaging. Carbon 66, 351-360 (2014).

21 Sahu, S., Behera, B., Maiti, T. K. \& Mohapatra, S. Simple one-step synthesis of highly luminescent carbon dots from orange juice: application as excellent bio-imaging agents. Chemical Communications 48, 8835-8837, doi:10.1039/C2CC33796G (2012).

22 Qu, S., Chen, H., Zheng, X., Cao, J. \& Liu, X. Ratiometric fluorescent nanosensor based on water soluble carbon nanodots with multiple sensing capacities. Nanoscale $\mathbf{5}$, 5514-5518, doi:10.1039/C3NR00619K (2013). 
23 Shi, W., Li, X. \& Ma, H. A Tunable Ratiometric pH Sensor Based on Carbon Nanodots for the Quantitative Measurement of the Intracellular $\mathrm{pH}$ of Whole Cells. Angewandte Chemie 124, 6538-6541, doi:10.1002/ange.201202533 (2012).

24 Cheng, H. et al. Graphene-Quantum-Dot Assembled Nanotubes: A New Platform for Efficient Raman Enhancement. ACS Nano 6, 2237-2244, doi:10.1021/nn204289t (2012).

25 Fan, Y. et al. Honeycomb architecture of carbon quantum dots: a new efficient substrate to support gold for stronger SERS. Nanoscale 4, 1776-1781, doi:10.1039/C2NR12015A (2012).

26 Zhang, H. et al. Fe 2 O 3/carbon quantum dots complex photocatalysts and their enhanced photocatalytic activity under visible light. Dalton Transactions 40, 10822-10825 (2011).

27 Bao, L. et al. Electrochemical tuning of luminescent carbon nanodots: from preparation to luminescence mechanism. Advanced Materials 23, 5801-5806 (2011).

28 Ghosh, S. et al. Photoluminescence of Carbon Nanodots: Dipole Emission Centers and Electron-Phonon Coupling. Nano Letters 14, 5656-5661, doi:10.1021/nl502372x (2014).

29 Wang, X., Zhang, J., Zou, W. \& Wang, R. Facile synthesis of polyaniline/carbon dot nanocomposites and their application as a fluorescent probe to detect mercury. RSC Advances 5, 41914-41919 (2015).

$30 \mathrm{Xu}, \mathrm{Q}$. et al. Synthesis, mechanistic investigation, and application of photoluminescent sulfur and nitrogen co-doped carbon dots. Journal of Materials Chemistry C 3, 9885-9893, doi:10.1039/C5TC01912E (2015).

$31 \mathrm{Xu}, \mathrm{Q}$. et al. Preparation of highly photoluminescent sulfur-doped carbon dots for Fe (III) detection. Journal of Materials Chemistry A 3, 542-546 (2015).

32 Vaseashta, A. \& Dimova-Malinovska, D. Nanostructured and nanoscale devices, sensors and detectors. Science and Technology of Advanced Materials 6, 312-318 (2005).

33 Li, H., Kang, Z., Liu, Y. \& Lee, S.-T. Carbon nanodots: synthesis, properties and applications. Journal of Materials Chemistry 22, 24230-24253, doi:10.1039/C2JM34690G (2012).

34 Wang, Q. et al. Hollow luminescent carbon dots for drug delivery. Carbon 59, 192-199 (2013). 
35 Thakur, M. et al. Antibiotic conjugated fluorescent carbon dots as a theranostic agent for controlled drug release, bioimaging, and enhanced antimicrobial activity. Journal of drug delivery 2014 (2014).

$36 \mathrm{Wu}, \mathrm{L}$. et al. Ultrasensitive electrochemiluminescence immunosensor for tumor marker detection based on nanoporous sliver@carbon dots as labels. Sensors and Actuators B: Chemical 186, 761-767 (2013).

37 Morfa, A. J., Rowlen, K. L., Reilly III, T. H., Romero, M. J. \& van de Lagemaat, J. Plasmon-enhanced solar energy conversion in organic bulk heterojunction photovoltaics. Applied Physics Letters 92, 013504 (2008).

38 Yatsui, T., Nomura, W. \& Ohtsu, M. Nanodot couplers provide efficient near-field energy transfer. Appl. Phys. Lett 27, 4583-4585 (2001).

39 Nomura, W., Ohtsu, M. \& Yatsui, T. Nanodot coupler with a surface plasmon polariton condenser for optical far/near-field conversion. Applied Physics Letters 86, 181108 (2005).

40 Yatsui, T., Kourogi, M. \& Ohtsu, M. Plasmon waveguide for optical far/near-field conversion. Applied Physics Letters 79, 4583-4585 (2001).

41 Yatsui, T., Kourogi, M. \& Ohtsu, M. Highly efficient excitation of optical near-field on an apertured fiber probe with an asymmetric structure. Applied Physics Letters 71, 1756-1758 (1997).

42 Bae, Y. M., Lee, K.-H., Yang, J. \& Heo, D. Fabrication of Gold Nanodot Array for the Localized Surface Plasmon Resonance. Journal of Nanomaterials 2014, 7, doi:10.1155/2014/175670 (2014).

43 Dong, C. \& Dinu, C. Z. Molecular trucks and complementary tracks for bionanotechnological applications. Current opinion in biotechnology 24, 612-619 (2013).

44 Ohler, B. Cantilever spring constant calibration using laser Doppler vibrometry. Review of Scientific Instruments 78, 063701 (2007).

45 Dinu, C. Z., Bale, S. S., Chrisey, D. B. \& Dordick, J. S. Manipulation of individual carbon nanotubes by reconstructing the intracellular transport of a living cell. Advanced Materials 21, 1182-1186 (2009).

46 Campbell, A. S. et al. A Systematic Study of the Catalytic Behavior at EnzymeMetal-Oxide Nanointerfaces. Nano LIFE 04, 1450005, doi:doi:10.1142/S1793984414500056 (2014). 
47 Ebenstein, Y., Mokari, T. \& Banin, U. Fluorescence quantum yield of CdSe/ZnS nanocrystals investigated by correlated atomic-force and single-particle fluorescence microscopy. Applied Physics Letters 80, 4033-4035 (2002).

48 Dantas, N. O., Silva, A., Silva, A. C. A. \& Neto, E. F. Atomic and magnetic force microscopy of semiconductor and semimagnetic nanocrystals grown in colloidal solutions and glass matrices. Optical Imaging: Technology, Methods and Applications 1, 109e132 (2012).

49 Xu, B. \& Tao, N. J. Measurement of single-molecule resistance by repeated formation of molecular junctions. Science 301, 1221-1223 (2003).

$50 \mathrm{Ma}, \mathrm{W}$. et al. Investigating electron-transfer processes using a biomimetic hybrid bilayer membrane system. Nat. Protocols 8, 439-450 (2013).

51 Wei, Y. et al. Electrochemical impedance determination of polychlorinated biphenyl using a pyrenecyclodextrin-decorated single-walled carbon nanotube hybrid. Chemical Communications 47, 5340-5342 (2011).

52 Gupta, R. K., Periyakaruppan, A., Meyyappan, M. \& Koehne, J. E. Label-free detection of C-reactive protein using a carbon nanofiber based biosensor. Biosensors and Bioelectronics 59, 112-119 (2014).

53 Sivam, A. S., Sun-Waterhouse, D., Perera, C. O. \& Waterhouse, G. I. N. Application of FT-IR and Raman spectroscopy for the study of biopolymers in breads fortified with fibre and polyphenols. Food Research International 50, 574-585 (2013).

54 Oancea, A. et al. Laboratory infrared reflection spectrum of carbon dioxide clathrate hydrates for astrophysical remote sensing applications. Icarus 221, 900-910 (2012).

55 Awwad, A., Salem, N. \& Abdeen, A. Green synthesis of silver nanoparticles using carob leaf extract and its antibacterial activity. Int $J$ Ind Chem 4, 1-6, doi:10.1186/2228-5547-4-29 (2013).

56 Mahdavi, M. et al. Synthesis, Surface Modification and Characterisation of Biocompatible Magnetic Iron Oxide Nanoparticles for Biomedical Applications. Molecules 18, 7533 (2013).

57 Yang, H., Yan, R., Chen, H., Lee, D. H. \& Zheng, C. Characteristics of hemicellulose, cellulose and lignin pyrolysis. Fuel 86, 1781-1788, (2007).

58 Deepa, M., Sharma, N., Agnihotry, S. A. \& Chandra, R. FTIR investigations on ionion interactions in liquid and gel polymeric electrolytes: LiCF3SO3-PC-PMMA. Journal of Materials Science 37, 1759-1765, doi:10.1023/A:1014921101649 (2002). 
59 Mamori, T., Matoba, M., Anzai, S. \& Fujimori, A. X-Ray Photoemission Spectroscopic Study of Impurity Effect and d-Band Formation Induced by the Substituted 4d Transition Metal Atoms in Ni1-xRhxS. Journal of the Physical Society of Japan 62, 1031-1036, doi:10.1143/JPSJ.62.1031 (1993).

60 Littlejohn, D. \& Chang, S.-G. An XPS study of nitrogen-sulfur compounds. Journal of Electron Spectroscopy and Related Phenomena 71, 47-50, (1995).

61 Barber, M., Connor, J. A., Derrick, L. M. R., Hall, M. B. \& Hillier, I. H. High energy photoelectron spectroscopy of transition metal complexes. Part 2.-Metallocenes. Journal of the Chemical Society, Faraday Transactions 2: Molecular and Chemical Physics 69, 559-562, doi:10.1039/F29736900559 (1973).

62 Rebholz, M., Matolin, V., Prins, R. \& Kruse, N. Methanol decomposition on oxygen precovered and atomically clean $\operatorname{Pd}(111)$ single crystal surfaces. Surface Science 251-252, 1117-1122 (1991).

63 Dennis, A. M. et al. X-ray photoelectron spectra of some dirhodium carboxylate complexes. Inorganica Chimica Acta 44, L139-L141, (1980).

64 Dai, Z., Hu, X., Wu, H. \& Zou, X. A label-free electrochemical assay for quantification of gene-specific methylation in a nucleic acid sequence. Chemical Communications 48, 1769-1771, doi:10.1039/C2CC15398J (2012).

65 Strauss, V. et al. Carbon Nanodots: Supramolecular Electron Donor-Acceptor Hybrids Featuring Perylenediimides. Angewandte Chemie International Edition 54, 8292-8297, doi:10.1002/anie.201502482 (2015).

66 Chen, M. et al. Sensitive electrochemical immunoassay of metallothionein-3 based on $\mathrm{K} 3[\mathrm{Fe}(\mathrm{CN}) 6]$ as a redox-active signal and $\mathrm{C}$-dots/Nafion film for antibody immobilization. The Analyst 138, 7341-7346, doi:10.1039/C3AN01351K (2013).

67 Sun, X., Wang, X. \& Jia, C. in Computer and Computing Technologies in Agriculture II, Volume 3 2283-2292 (Springer, 2009).

68 Sun, X. \& Wang, X. Acetylcholinesterase biosensor based on prussian blue-modified electrode for detecting organophosphorous pesticides. Biosensors and Bioelectronics 25, 2611-2614, (2010).

69 Li, J. et al. Nonenzymatic glucose sensor based on a glassy carbon electrode modified with chains of platinum hollow nanoparticles and porous gold nanoparticles in a chitosan membrane. Microchim Acta 172, 163-169, doi:10.1007/s00604-010-0485-z (2011). 
$70 \mathrm{Xu}, \mathrm{H} ., \mathrm{Dai}, \mathrm{H}$. \& Chen, G. Direct electrochemistry and electrocatalysis of hemoglobin protein entrapped in graphene and chitosan composite film. Talanta 81, 334-338 (2010).

71 Haynes, W. M. CRC handbook of chemistry and physics. (CRC press, 2014).

72 Laviron, E. General expression of the linear potential sweep voltammogram in the case of diffusionless electrochemical systems. Journal of Electroanalytical Chemistry and Interfacial Electrochemistry 101, 19-28 (1979).

73 Laviron, E., Roullier, L. \& Degrand, C. A multilayer model for the study of space distributed redox modified electrodes: Part II. Theory and application of linear potential sweep voltammetry for a simple reaction. Journal of Electroanalytical Chemistry and Interfacial Electrochemistry 112, 11-23 (1980).

74 Nogales, E. Structural Insights into Microtubule Function. Annual Review of Biochemistry 69, 277-302, doi:doi:10.1146/annurev.biochem.69.1.277 (2000).

75 Dinu, C. Z., Bale, S. S., Zhu, G. \& Dordick, J. S. Tubulin Encapsulation of Carbon Nanotubes into Functional Hybrid Assemblies. Small 5, 310-315, doi:10.1002/smll.200801434 (2009).

76 Dong, C. et al. Exposure to carbon nanotubes leads to changes in the cellular biomechanics. Advanced healthcare materials 2, 945-951 (2013).

77 Eldawud, R., Wagner, A., Dong, C., Rojansakul, Y. \& Zoica Dinu, C. Electronic platform for real-time multi-parametric analysis of cellular behavior post-exposure to single-walled carbon nanotubes. Biosensors and Bioelectronics 71, 269-277 (2015).

78 Ionov, L., Bocharova, V. \& Diez, S. Biotemplated synthesis of stimuli-responsive nanopatterned polymer brushes on microtubules. Soft Matter 5, 67-71, doi:10.1039/B813295J (2009).

79 Acres, R. G. et al. Molecular Structure of 3-Aminopropyltriethoxysilane Layers Formed on Silanol-Terminated Silicon Surfaces. The Journal of Physical Chemistry C 116, 6289-6297, doi:10.1021/jp212056s (2012).

80 Hayat, M. Fixation for electron microscopy. (Elsevier, 2012).

81 Sheldon, R. A. Characteristic features and biotechnological applications of cross-linked enzyme aggregates (CLEAs). Applied microbiology and biotechnology 92, 467-477 (2011).

82 Walt, D. R. \& Agayn, V. I. The chemistry of enzyme and protein immobilization with glutaraldehyde. TrAC Trends in Analytical Chemistry 13, 425-430 (1994). 
83 Tashima, T., Imai, M., Kuroda, Y., Yagi, S. \& Nakagawa, T. Structure of a new oligomer of glutaraldehyde produced by aldol condensation reaction. The Journal of Organic Chemistry 56, 694-697 (1991).

84 Turner, D., Chang, C., Fang, K., Cuomo, P. \& Murphy, D. Kinesin Movement on Glutaraldehyde-Fixed Microtubules. Analytical Biochemistry 242, 20-25 (1996).

85 Kiernan, J. A. Formaldehyde, formalin, paraformaldehyde and glutaraldehyde: what they are and what they do. Microscopy Today 1 (2000).

86 Bekale, L., Agudelo, D. \& Tajmir-Riahi, H. A. Effect of polymer molecular weight on chitosan-protein interaction. Colloids and Surfaces B: Biointerfaces 125, 309-317, (2015).

87 Kafka, J., Pänke, O., Abendroth, B. \& Lisdat, F. A label-free DNA sensor based on impedance spectroscopy. Electrochimica Acta 53, 7467-7474 (2008).

88 Moulton, S. E., Barisci, J. N., Bath, A., Stella, R. \& Wallace, G. G. Studies of double layer capacitance and electron transfer at a gold electrode exposed to protein solutions. Electrochimica Acta 49, 4223-4230 (2004).

89 Liu, Q. et al. Graphene-modified nanostructured vanadium pentoxide hybrids with extraordinary electrochemical performance for Li-ion batteries. Nature communications 6 (2015).

90 Wang, M. et al. Application of impedance spectroscopy for monitoring colloid Au-enhanced antibody immobilization and antibody-antigen reactions. Biosensors and Bioelectronics 19, 575-582 (2004).

91 Zhang, S., Wu, Z., Shen, G. \& Yu, R. A label-free strategy for SNP detection with high fidelity and sensitivity based on ligation-rolling circle amplification and intercalating of methylene blue. Biosensors and Bioelectronics 24, 3201-3207, (2009).

92 Kissinger, P. T. \& Heineman, W. R. Cyclic voltammetry. Journal of Chemical Education 60, 702 (1983).

93 Xie, C. et al. Core-Shell Heterojunction of Silicon Nanowire Arrays and Carbon Quantum Dots for Photovoltaic Devices and Self-Driven Photodetectors. Acs Nano 8, 4015-4022, doi:10.1021/nn501001j (2014).

94 Bian, J. C. et al. Carbon Dot Loading and TiO2 Nanorod Length Dependence of Photoelectrochemical Properties in Carbon Dot/TiO2 Nanorod Array Nanocomposites. Acs Appl Mater Inter 6, 4883-4890, doi:10.1021/am4059183 (2014). 


\section{Supporting Information}

\section{Characterization of S-doped C-dots}

The morphology of as-prepared S-doped C-dots was investigated using high-resolution transmission electron microscopy (HRTEM; Model JEM-2100). First, the samples (1 mL of purified S-doped C-dots solution was diluted into $10 \mathrm{~mL}$ of water), dropped onto copper wire meshes, and dried for $20 \mathrm{~min}$ at $55^{\circ} \mathrm{C}$. An accelerated voltage of $200 \mathrm{KV}$ was used for analyses. Sample's sizes are revealed in Figure S1.

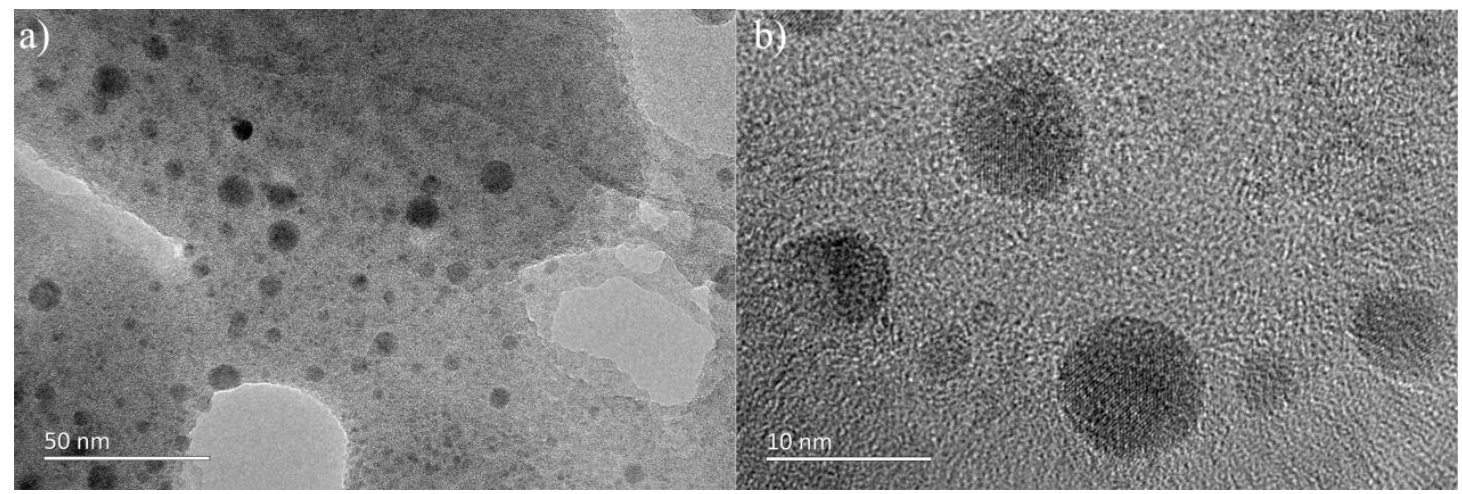

Figure S1: HRTEM images of S-doped C-dots. Scale bars: a) $50 \mathrm{~nm}$, b) $10 \mathrm{~nm}$.

\section{High resolution AFM image of the S-doped C-dots}

In order to provide details of the hybrids structure, high-resolution AFM images were obtained. The experimental details were exactly the same as described in the manuscript. Briefly, contact mode Atomic Force Microscopy (AFM, Asylum Research, USA) with a silicon nitride tip (TR-400PB, Asylum Research, USA) in solution was used. The trigger force was kept constant at $3 \mathrm{nN}$ while the spring constant of the cantilever was measured before each experiment using established method. 


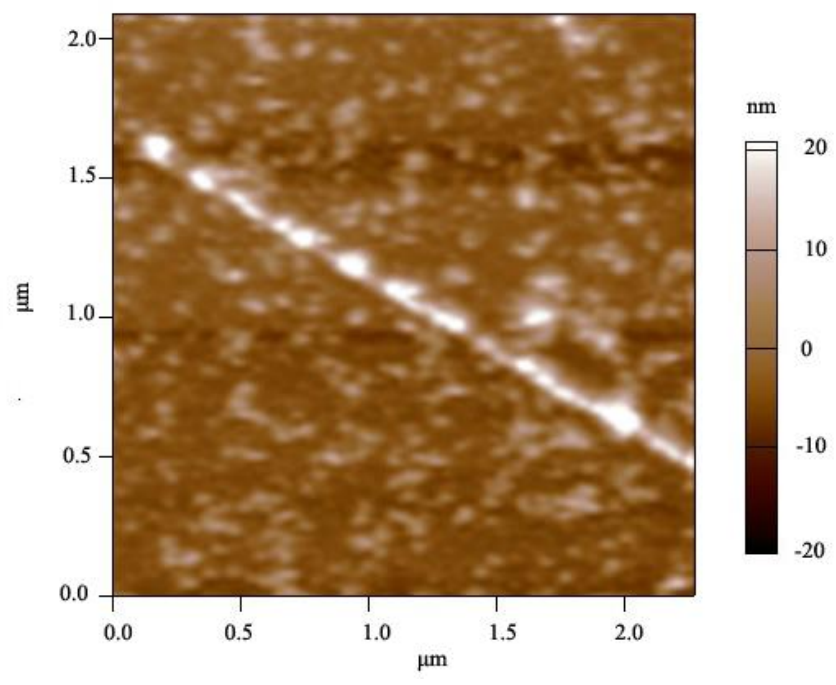

Figure S2: High resolution AFM image of the S-doped C-dots hybridized microtubule showing S-doped C-dots incorporated into the polymerized hybrid as a bead-like geometry.

\section{Evaluate the effect of chitosan on tubulin self-assembly}

The polymerization solution was obtained by vortexing $5 \mu \mathrm{L} 100 \mathrm{mM}$ magnesium chloride $\left(\mathrm{MgCl}_{2}\right.$, Fisher Scientific, USA), with $6 \mu \mathrm{L}$ dimethyl sulfoxide (DMSO, 99.7\%, Fisher Scientific, USA), $5 \mu \mathrm{L} 25 \mathrm{mM}$ guanosine-5'-triphosphate (GTP, Sigma, USA) and $9 \mu \mathrm{L}$ BRB80 buffer (formed from a mixture of $80 \mathrm{mM}$ piperazine-N,N'-bis(2-ethanesulfonic acid buffer, $1 \mathrm{mM} \mathrm{MgCl} 2$ and $1 \mathrm{mM}$ ethylene glycol tetraacetic acid (EGTA), $\mathrm{pH}$ 6.8; all reagents were purchased from Fisher Scientific, USA).

Microtubule-chitosan were intended to be obtained upon mixing10 $\mu \mathrm{L}$ of $4 \mathrm{mg} / \mathrm{mL}$ rhodamine-biotin-tubulin with $10 \mu \mathrm{L} \mathrm{50 \%}$ (wt) chitosan and initiating their polymerization. Specifically, $5 \mu \mathrm{L}$ polymerization solution (see above) was injected into $20 \mu \mathrm{L}$ of the rhodamine-biotin-tubulin-chitosan mixture and incubated at $37^{\circ} \mathrm{C}$ for $30 \mathrm{~min}$. To stabilize any of the resulting structures, the solution was dispersed in $1 \mathrm{~mL} \mathrm{BRB} 80$ buffer containing 10 $\mu \mathrm{M}$ taxol (Fisher Scientific, USA). The solution was kept at room temperature for experimental usage.

For the electrochemical impedance spectroscopy (EIS) analyses, $20 \mu \mathrm{L}$ of the obtained "microtubule-chitosan" solution was dropped onto a cleaned electrode and incubated overnight under vacuum (same procedure as listed in the materials and methods). The EIS analyses have been performed in $50 \mathrm{mM}$ potassium ferricyanide $\left(\mathrm{K}_{3} \mathrm{Fe}(\mathrm{CN})_{6}\right.$; Fisher Scientific, USA) in BRB80 buffer containing $10 \mu \mathrm{M}$ taxol. The supporting electrolyte used in 
our experiments is $10 \mathrm{mM} \mathrm{NaCl}$.

Analyses reveal no major differences between the two curves (Figure S3).

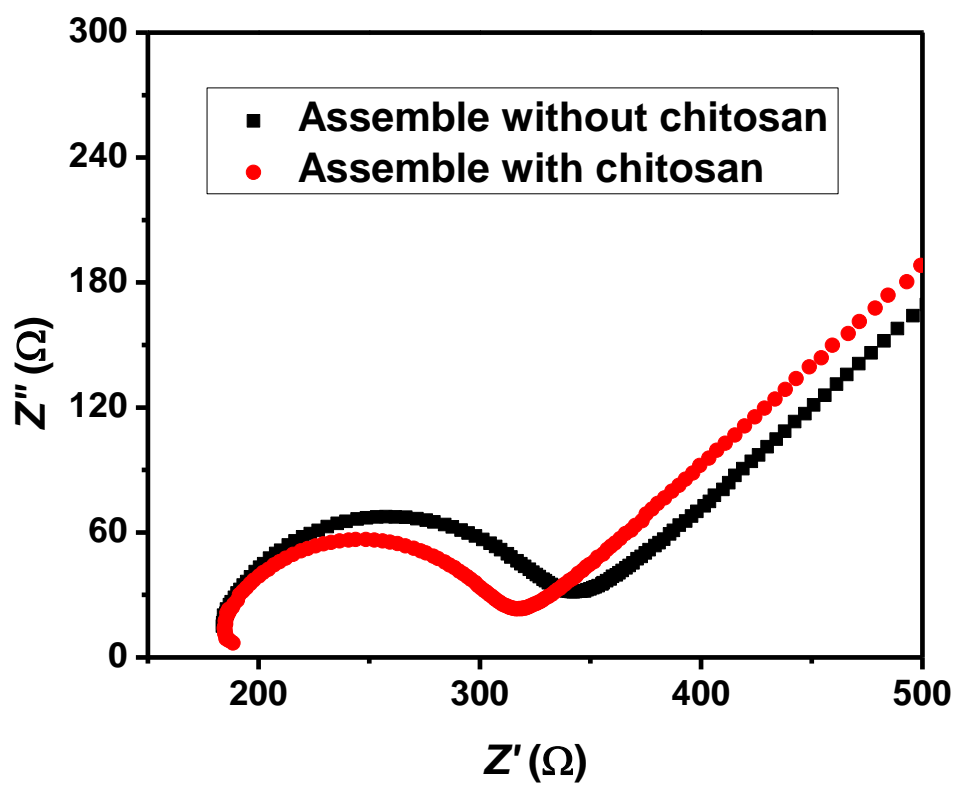

Figure S3: EIS graph of the modified electrode. Black curve: microtubule-chitosan/Au (the tubulin assembled in the presence of chitosan). Red curve: microtubule/chitosan/Au.

\section{Evaluate the change in impedance on the chitosan membrane}

For the bio-hybrid synthesis, also called hybrid microtubule, first biotin-tubulin-S-doped C-dots-conjugates were formed using non-specific binding of biotin-tubulin onto S-doped C-dots scaffolds as described in the paper. Briefly, $1 \mu \mathrm{L} 6 \mathrm{mg} / \mathrm{mL}$ synthesized S-doped C-dots were injected into a $600 \mu \mathrm{L}$ eppendorf tube containing $5 \mu \mathrm{L}$ of $4 \mathrm{mg} / \mathrm{mL}$ biotin-tubulin and the mixture was incubated for $2 \mathrm{~h}$ at $200 \mathrm{rpm}$ in an ice bath. Subsequently, $5 \mu \mathrm{L}$ of $4 \mathrm{mg} / \mathrm{mL}$ free biotin-tubulin was mixed with the biotin functionalized tubulin- S-doped C-dots conjugates and an additional $2.5 \mu \mathrm{L}$ microtubule polymerization solution, and subjected to 37 ${ }^{\circ} \mathrm{C}$ for 30 min. When time elapsed, the hybrids were stabilized in BRB80 buffer containing 10 $\mu \mathrm{M}$ taxol.

Synthesized S-doped C-dots hybridized microtubules were spun down using high-speed centrifuge (30000 rpm for $10 \mathrm{~min}$ at room temperature). The supernatant was removed carefully and the pellet was re-suspended in $1 \mathrm{~mL} \mathrm{BRB80}$ buffer containing $10 \mu \mathrm{M}$ taxol.

The synthesized microtubule and hybrids (before and after the centrifugation) were 
immobilized onto the electrode using the methods described in the manuscript. EIS analyses are presented in Figure S4; minor changes (not statistically relevant) have been observed most likely associated with changes in the microtubule/hybrid length known to occur because of the mechanical stress imposed by centrifugation.

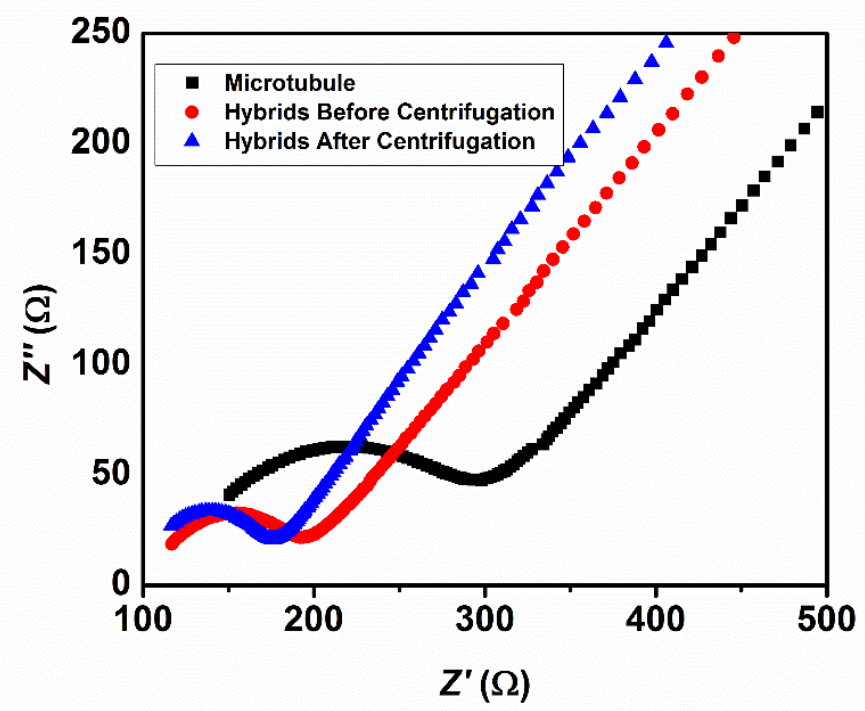

Figure S4: EIS graph of the modified electrode. Black curve: Microtubule/Chitosan/Au. Red curve: S-doped C-dots hybridized microtubule/Chitosan/Au. Blue curve, Centrifuged S-doped C-dots hybridized microtubule/Chitosan/Au 
Chapter 4: Biological self-assembly and recognition used to synthesize and surface guide next generation of hybrid materials

\begin{abstract}
Free-standing, high aspect ratio sulfur-doped carbon nanodots-based hybrid nanowires with a microtubular aspect were synthesized using self-recognition and self-assembly processes of tubulin, a biological molecule precursor of the cytoskeletal cellular and structural microtubule filaments. Physico-chemical characterizations (e.g., morphology, diameter, spectral characteristics etc.) of tsuch user-synthesized hybrid bio-nanowires were performed using classical atomic and spectroscopic techniques, while bioactivity and functionality testing was demonstrated by mimicking cellular transport based on kinesin, a motor protein capable to recognize, bind onto and move on the microtubules. Our results indicate that user-synthesized hybrid nanowires could be manipulated in vitro under constant chemical energy of adenosine triphosphate (ATP) and have the potential to be implemented in the next generation of synthetic applications from drug delivery to diagnosis systems, and photocatalytic to optical devices.
\end{abstract}




\section{Introduction}

The ultrahigh surface-to-volume ratios of synthetic nanowires-like geometries developed through hard template ${ }^{1}$, electrochemical ${ }^{2}$, vapor deposition ${ }^{3}$ or vapor-liquid-solid (VLS) ${ }^{4}$ methods have found applications in a variety of fields from one dimension electronics ${ }^{5}$ to sensor-based devices ${ }^{6}$. Compared with their bulk counterparts, such wire-like geometries show surface relaxation ${ }^{7}$ and improved kinetics effects ${ }^{8}$ while allowing the combination of non-lattice-matched materials ${ }^{9}$ with high aspect ratios and dipolar magnetic properties to influence their ability to transfer energy at interfaces ${ }^{10}$. Single $\mathrm{ZnO}$ nanowires obtained through chemical vapor deposition were, for instance, used in a gas sensor system to detect hydrogen at low concentration ${ }^{11}$, while a $\mathrm{Co}_{2} \mathrm{Si}_{-}-\mathrm{Al}_{2} \mathrm{O}_{3}$ core-shell nanowire obtained through the same method was used as a gate in a high-speed graphene transistor to ensure that the edges of the source, the drain and the gate electrodes gave minimum access resistance in a device $^{12}$. Similarly, C-based nanowires have been proposed for electronic ${ }^{13}$ and for energy generation templates ${ }^{14}$, for photovoltaic devices assembly ${ }^{15}$ or to be used in photocatalysis ${ }^{16}$, storage $^{17}$, lighting systems ${ }^{18}$, bioimaging ${ }^{19}$, and nanosensors ${ }^{20}$ formation. Their tunable luminescence emission $^{21}$, high stability against photobleaching and blinking ${ }^{22}$, also allowed the C-based templates to be used as environmentally friendly fluorescence probes for rapid detection of $\mathrm{Hg}^{2+}$ and bio-thiols, all with high sensitivity and selectivity ${ }^{23}$. Cao L et al., ${ }^{24}$ for instance, used C-dots as photocatalysts for $\mathrm{CO}_{2}$ conversion to drive efficient photocatalysis.

The implementation of such nanowires is however hindered by the complex and laborious manufacturing processes, as well as the limited ability they have to be placed after synthesis onto precise nanosize locations, all under user-control. Isolated studies showed that while hard templates ${ }^{1}$ could be used for various geometries formation at relatively high temperatures, the hard treatment required to remove such geometries for placement could possibly lead to defects in the formed nanowire' structures ${ }^{25}$. Complementary, while electrospinning can be used to synthesize long nanowires (up to millimeters $\operatorname{long}^{26}$ ), the synthesized templates are hard to separate from the soluble polymer used as support ${ }^{27}$. Moreover, while methods such as atomic force microscopy ${ }^{28}$ or optical trapping ${ }^{29}$ could be employed for individual physical manipulation and user-placement of individual nanowires, such methods could only allow displaying one template at a time and onto a localized nanosize position respectively. Before the next generation of template nanowires could be largely implemented, controlled nanomanufacturing technologies of both the surface characteristics and size, as well as user-directed nanoscale placement strategies need be 
proposed.

Herein we hypothesize that biological self-assembly and self-recognition could be used to direct synthesis and user-placement of the next generation of C-based nanowires of controlled geometries that could possibly ensure effective charge transfer at their interfaces ${ }^{30}$. To demonstrate this hypothesis, we exploited the ability of cellular protein tubulin to serve as supporting polymerization unit of linear microtubule-like structure when immobilized onto C-based quantum dots templates and in a synthetic environment. Microtubules are intercellular cytoskeletal filaments involved in cellular activities from division to ciliary functions $^{31}$, known to provide structure and spatial organization of the cell ${ }^{32}$, as well as serve as tracks for molecular motors used in trafficking of vesicles ${ }^{33}$, $\mathrm{DNA}^{34}$ or sensorial molecules $^{35}$. C-based quantum dots were chosen as templates based on their known regular geometries and known conductivity, as well as ease of synthesis ${ }^{30,36}$.

Our results demonstrate that microtubule-like linear structures of hybrids containing C-based nanomaterials could be achieved in a controllable fashion; further our results show that large surface-to-volume aspect ratios are formed by implementing biological functions to non-biological materials. By exploiting the self-recognition ability of kinesin proteins, known to transport cargo onto microtubules tracks in the cells to test hybrids guiding under user-control and under the chemical energy of adenosine triphosphate (ATP), we also provide a viable strategy to create the next generation of smart materials that could be placed onto nanosize locations, all under user-controlled approaches, to be further possibly exploited for sensing ${ }^{37}$, imaging $^{38}$ and macromolecular display ${ }^{39}$ applications. 


\section{Materials and Methods}

\section{Expression of fluorescently labeled kinesin molecular motor (dmKHC-EGFP)}

Package plasmid F-drosophila melanogaster kinesin heavy chain-enhanced green fluorescent protein (pPF_dmKHC-EGFP) encoding for the dmKHC-EGFP (molecular weight $91.7 \mathrm{kDa}$ ) and consisting of the drosophila melanogaster kinesin delta tail linked to the C-terminal end to the EGFP his-tagged on the C-terminus was obtained at West Virginia University (WVU). The coding sequence for dmKHC was copied by Polymerase Chain Reaction (PCR) from the pPK124 plasmid, a kind gift of Prof. Jonathan Howard, Yale University. Briefly, a set of primers of known sequences (CAAAGGAGATATACATATGAGCGCAGAACGAGAAATTCC and CTCGCCCTTGCTCAC.GCTCCCACGCGGAACAAG respectively) were used; pPF_EGFP plasmid was a pTriEx-4 plasmid (Novagen, part of EMD Millipore, USA) carrying the coding sequence for a C-terminal his-tagged EGFP protein. The pPF_EGFP plasmid was first linearized by treatment with the NcoI endonuclease (New England Biolabs, USA). Both the linearized pPF_EGFP plasmid and the pPK124 PCR amplicon were then gel purified and assembled using the Gibson assembly kit (New England Biolabs, USA) and following manufacturer protocols ${ }^{40}$. The assembled DNA sequences were subsequently introduced into E. Coli strain Stb14 (Invitrogen, Fisher Scientific, USA); clones were screened by endonuclease treatment and small-scale protein expression followed by standard lab purification to obtain the protein.

The pPF_dmKHC-EGFP plasmid was transformed into the E. Coli strain BL21(DE3) pLysS (Stratagene, Agilent Technologies, USA). Protein expression was induced with Isopropyl $\beta$-D-1-thiogalactopyranoside (1.0 mM, IPTG, Fisher Scientific, USA) at $16{ }^{\circ} \mathrm{C}$ for $18 \mathrm{~h}$. The cell pellet was subsequently re-suspended into ice-cold lysis buffer containing tris(hydroxymethyl)aminomethane hydrochloride (50 mM, pH 8.0, Tris/HCl, Fisher Scientific, USA), sodium chloride (50 mM, NaCl, Fisher Scientific, USA), magnesium chloride ( $2 \mathrm{mM}$, $\mathrm{MgCl}_{2}$, Fisher Scientific, USA), adenosine 5'-triphosphate (0.1 mM, ATP, Sigma, USA), Triton X-100 (2\%, Fisher Scientific, USA), $\beta$-mercaptoethanol (10 mM, Fisher Scientific, USA), lysozyme $(0.4 \mathrm{mg} / \mathrm{ml})$, ethylenediaminetetraacetic acid (EDTA)-free protease inhibitor cocktail (Biotool.com, TX, USA), phenylmethylsulfonyl fluoride (1 mM, PMSF, Sigma, USA), trehalose ( $8 \%$, Fisher Scientific, USA), and Pierce Universal Nuclease (10 U/ml, Fisher Scientific, USA), at lysis buffer $(10 \mathrm{~mL})$ per $1 \mathrm{~g}$ of cell pellet. Cellular debris was removed by centrifugation (performed at $20000 \mathrm{~g}, 4{ }^{\circ} \mathrm{C}, 10 \mathrm{~min}$, while the clear lysate was 
supplemented with $\mathrm{NaCl}(4 \mathrm{M})$ to bring the final $\mathrm{NaCl}$ concentration to $0.5 \mathrm{M}$.

Expressed protein was purified in series using two Bio-scale mini IMAC cartridges (Bio-Rad Laboratories Inc., USA) installed in a BioLogic DuoFlow chromatography system (Bio-Rad Laboratories Inc., USA) by running two buffers, i.e., the wash buffer (sodium phosphate $\left(50 \mathrm{mM}, \mathrm{Na}_{2} \mathrm{PO}_{4}\right), \quad \mathrm{NaCl}(0.3 \mathrm{M}), \mathrm{MgCl}_{2} \quad\left(\begin{array}{lll}1 & \mathrm{mM}\end{array}\right), \quad$ ATP $\left(\begin{array}{lll}10 & \mu \mathrm{M}\end{array}\right)$, $\beta$-mercaptoethanol (5 mM), glycerol (10\%, Fisher Scientific, USA), pH 8.0) and the elution buffer (wash buffer with imidazole (0.5 M, Fisher Scientific, USA) respectively. The protein was then eluted by imidazole gradient and concentrated to $1 \mathrm{~mL}$.

A second chromatographic step was applied for further protein purification using a Superdex 200 10/300 GL gel-filtration column (GE Healthcare Life Sciences, USA) equilibrated with storage buffer (imidazole (100 mM), $\mathrm{NaCl}$ (300 mM), $\mathrm{MgCl}_{2}$ (1 mM), ATP $(10 \mu \mathrm{M}), 1,4-$ dithiothreitol (1.0 mM, DTT, Fisher Scientific, USA), and 10\% sucrose (Fisher Scientific, USA, pH 7.0). Lastly, the protein concentration was estimated using the Coomassie protein assay and bovine gamma globulin standard (reagents from Fisher Scientific, USA), while the purification process was monitored by classic SDS-PAGE (Novagen, USA).

\section{Synthesis of the sulfur-doped carbon nanodots}

Sulfur-doped carbon nanodots (S-doped C-dots) were synthesized using an established hydrothermal method ${ }^{36}$. Briefly, sodium citrate $(0.1 \mathrm{M}, 25 \mathrm{~mL})$ and sodium thiosulfate solutions (Tianjin Guangfu Technology Development Co., Ltd., China) were added into a Teflon-lined stainless steel autoclave $(50 \mathrm{~mL}$, Fuzhou Keleisi Test Equipment Co., Ltd., China) and the autoclave was incubated at $200{ }^{\circ} \mathrm{C}$ for $6 \mathrm{~h}$. The resulting products were filtered through a $0.22 \mu \mathrm{m}$ membrane (Spectrum Laboratories, Inc. USA). The products were washed with ethanol (5 mL, 90\%, Fisher Scientific, USA) and deionized (DI) water. Washed products were filtered through a $0.22 \mu \mathrm{m}$ filtration membrane and dried at room temperature over night.

\section{Synthesis of tubulin-S-doped C-dots conjugates}

Tubulin-S-doped C-dots-conjugates were formed using non-specific binding of biotin rhodamine labeled tubulin (Cytoskeleton Inc, USA) onto S-doped C-dots scaffolds ${ }^{41}$. Briefly, synthesized S-doped C-dots $(1 \mu \mathrm{L}$ of $6 \mathrm{mg} / \mathrm{mL})$ were injected into an eppendorf tube containing biotin rhodamine labeled tubulin $(5 \mu \mathrm{L}$ of $4 \mathrm{mg} / \mathrm{mL})$ and the mixture was 
subsequently incubated for $2 \mathrm{~h}$ at $200 \mathrm{rpm}$ in an ice bath.

\section{Spectroscopic evaluations}

The absorbance of the S-doped C-dots and tubulin-S-doped C-dots-conjugates was monitored on a UV-Vis spectrophotometer (Thermo Scientific EVO300) at $305 \mathrm{~nm}$. For this, both S-doped C-dots and tubulin-S-doped C-dots-conjugates respectively were dispersed in buffered phosphate saline (100 mM, PBS, pH 7.4, formed from a mixture of monopotassium phosphate, $\mathrm{KH}_{2} \mathrm{PO}_{4}$, and dipotassium hydrogen phosphate, $\mathrm{K}_{2} \mathrm{HPO}_{4}$, purchased from Fisher Scientific, USA). The concentrations of the S-doped C-dots and tubulin in the tubulin-S-doped C-dots-conjugates were $10 \mathrm{mg} / \mathrm{mL}$ and $1.5 \mathrm{mg} / \mathrm{mL}$ respectively. The secondary structure of the protein before and after binding to the S-doped C-dots (concentration of $500 \mu \mathrm{g} / \mathrm{mL}$ ) was evaluated using circular dichroism (CD) performed on a Jasco 810 Circular Dichroism Spectroploarimeter (Jasco Inc). The scanning range was in between 190 to $260 \mathrm{~nm}$ with a scanning speed of $50 \mathrm{~nm} / \mathrm{min}$; the bandwidth was $1 \mathrm{~nm}$.

\section{Synthesis of microtubule and S-doped C-dots hybrid microtubule}

Microtubules were synthesized according to established protocols ${ }^{30}$. Briefly, $\mathrm{MgCl}_{2}(5$ $\mu \mathrm{L}, 100 \mathrm{mM}$ ), with dimethyl sulfoxide (6 $\mu \mathrm{L}$, DMSO, 99.7\%, Fisher Scientific, USA), guanosine-5'-triphosphate ( $5 \mu \mathrm{L}, 25 \mathrm{mM}$, GTP, Sigma, USA) and BRB80 buffer $(9 \mu \mathrm{L}, \mathrm{pH}$ 7.3 , formed from a mixture of piperazine-N,N'-bis(2-ethanesulfonic acid buffer $(80 \mathrm{mM})$, $\mathrm{MgCl}_{2}(1 \mathrm{mM})$ and ethylene glycol tetraacetic acid (1 mM, EGTA); all reagents were purchased from Fisher Scientific, USA respectively) were vortexed to generate a microtubule polymerization solution. To initiate microtubule polymerization, the polymerization solution $(2.5 \mu \mathrm{L})$ and biotin rhodamine tubulin $(10 \mu \mathrm{L}, 4 \mathrm{mg} / \mathrm{mL})$ were mixed and subsequently incubated at $37{ }^{\circ} \mathrm{C}$ for $30 \mathrm{~min}$. To stabilize the resulting microtubules, the solution was dispersed in BRB80 buffer (1 mL) containing paclitaxel (10 $\mu \mathrm{M}$, Fisher Scientific, USA). The stabilized microtubules were stored at room temperature for usage.

For S-doped C-dots hybrid microtubule (hybrids for short) synthesis, tubulin-S-doped C-dots conjugates were first formed as described above. Secondly, hybrids were assembled by mixing the tubulin-S-doped C-dots conjugates with free biotin rhodamine labeled tubulin in solution $(5 \mu \mathrm{L}$ of $4 \mathrm{mg} / \mathrm{mL})$ and an additional microtubule polymerization solution $(2.5 \mu \mathrm{L})$, and subjecting the final mixture to $37{ }^{\circ} \mathrm{C}$ for $30 \mathrm{~min}$. Free tubulin, free S-doped C-dots or free S-doped C-dots conjugates were removed by centrifugation after the hybrids were formed 
with centrifugation being performed at $30000 \mathrm{rpm}$ on an Allegra 64R centrifuge (Beckman Coulter, USA) operating for $10 \mathrm{~min}$.

When the time elapsed, the solution was dispersed into BRB80 buffer $(1 \mathrm{~mL})$ containing paclitaxel $(10 \mu \mathrm{M})$ to stabilize any of the formed structures; the stabilized structures were subsequently stored at room temperature for further experimental use.

\section{Kinesin accumulation}

Microtubules or hybrids solution were mixed with lab-synthesized kinesin in order to evaluate the ability of kinesin to recognize structures' specific sites. Briefly, kinesin $(8 \mu \mathrm{L}, 10$ $\mu \mathrm{g} / \mathrm{mL}$ ) was mixed with adenylyl-imidodiphosphate ( $2 \mu \mathrm{L}, 20 \mathrm{mM}$, AMP-PNP, Sigma, USA) and incubated for $1 \mathrm{~h}$ at $4{ }^{\circ} \mathrm{C}$. Subsequently, the mixture was mixed with microtubules or hybrids solutions $(20 \mu \mathrm{L})$ and BRB80 buffer $(10 \mu \mathrm{L})$ containing paclitaxel $(10 \mu \mathrm{M})$ for a final volume of $40 \mu \mathrm{L}$, and then incubated at room temperature for another $30 \mathrm{~min}$. Any unbound kinesin was removed upon spinning down the microtubules or hybrids at 30,000 rpm for 10 min. The supernatant containing such suspended kinesin was evaluated using a fluorescence microscope (Nikon, USA) and a 100x objective of NA=1.4. The washing procedure was repeated until no free kinesin was observed. The pellet was subsequently dissolved in BRB80 (40 $\mu \mathrm{L})$ containing paclitaxel $(10 \mu \mathrm{M})$ and used immediately after preparation.

\section{Mica surface functionalization}

Mica sheets were first washed with DI water, ethanol, and again with DI water, and subsequently dried under vacuum overnight and at room temperature. The cleaned mica was subsequently functionalized with 3-aminopropyltriethoxysilane (APTES, Fisher Scientific, USA; $20 \mu \mathrm{L}, 99 \%$ ) by incubation at room temperature for $15 \mathrm{~min}$. After incubation, mica was rinsed thoroughly with toluene (99.8\%, Fisher Scientific, USA) to remove excess APTES and then with DI water; any remaining solution was removed under vacuum.

\section{Atomic Force Microscopy (AFM) evaluation}

Biological-based samples (microtubules, hybrids, kinesin-microtubule conjugates and kinesin-hybrid conjugates) were analyzed for their physical characteristics using Atomic Force Microscopy (AFM) and established protocols ${ }^{30}$. Briefly, individual sample $(19.5 \mu \mathrm{L})$ was mixed with glutaraldehyde $(0.5 \mu \mathrm{L}, 20 \%$, Fisher Scientific, USA) and shaken gently. Subsequently, the mixture was dropped onto APTES functionalized mica surfaces and 
incubated for $1 \mathrm{~h}$ at room temperature. After the incubation, the surface was washed with BRB80 buffer $(40 \mu \mathrm{L})$ containing paclitaxel $(10 \mu \mathrm{M})$ for at least 3 times. AFM images were collected immediately using contact mode in solution. For these measurements the cantilever's spring constant was calibrated using the thermal noise method ${ }^{42}$; the scan rate of the tip was fixed at $0.5 \mathrm{~Hz}$ for all the experiments.

The diameter distribution of 83 individual microtubules, 70 individual kinesin-microtubule conjugates measured on the kinesin and 82 individual kinesin-microtubule conjugates measured randomly were collected from 25 and 23 independent images respectively. The diameter distribution of 68 individual hybrids, 66 individual kinesin-hybrid conjugates measured on the kinesin and 80 individual kinesin-hybrid conjugates measured randomly were collected from 20 and 22 independent images respectively.

\section{Preparation of the microscopy flow cell}

Flow cells were prepared using a glass slide, a cover glass (Corning, USA) and double-sided tape. Prior to the formation of the flow cell, the glasses were sonicated in ethanol and DI water for $30 \mathrm{~min}$ each. Subsequently, the glasses were treated with Piranha solution (containing sulfuric acid $\left(96.4 \%, \mathrm{H}_{2} \mathrm{SO}_{4}\right)$,) and hydrogen peroxide $\left(30 \%, \mathrm{H}_{2} \mathrm{O}_{2}\right)$, both reagents from Fisher Scientific, USA) in a $3: 1$ (v:v) for $1 \mathrm{~h}$ at $60{ }^{\circ} \mathrm{C}$, rinsed thoroughly with DI water and blown dry. Such cleaned surfaces were used directly in the gliding assay.

For the stepping assay, the cleaned glasses underwent further treatment to obtain a hydrophobic surface. Briefly, the cleaned glass was immersed into trichloroethylene (TCE, Fisher Scientific, USA) solution which containing 4,4'-Diaminodiphenylsulfone $(0.05 \%$, DDS, Fisher Scientific, USA,) for $1 \mathrm{~h}$ and then sonicated in methanol (99.8\%, Fisher Scientific, USA) for $5 \mathrm{~min}, 15 \mathrm{~min}$ and $30 \mathrm{~min}$ respectively to remove any unbound TCE. Lastly, the glasses were blown dry and used to assemble flow cells as described above.

\section{Kinesin-microtubule motility assays}

Motility assay was performed following established protocols ${ }^{43}$; specifically for the gliding assay, casein (20 $\mu \mathrm{L}, 0.5 \mathrm{mg} / \mathrm{mL}$, Fisher Scientific, USA) in BRB80 was added into the flow cell (assembled as previously indicated) and incubated for $5 \mathrm{~min}$ at room temperature. After that, kinesin solution $(20 \mu \mathrm{L}, 10 \mu \mathrm{g} / \mathrm{mL})$ with casein $(0.5 \mathrm{mg} / \mathrm{mL})$ and $\operatorname{MgATP}(1 \mathrm{mM})$ in BRB80 buffer was also added and incubated for another $5 \mathrm{~min}$ at room 
temperature. Lastly, 'modified' microtubule solution $(20 \mu \mathrm{L}$, BRB80 buffer which containing microtubules or the equivalent concentration of the hybrids (32 nM), MgATP (1mM), d-glucose (20 mM, Fisher Scientific, USA), glucose oxidase (0.02 mg/mL, Sigma, USA), catalase $(0.8 \mathrm{mg} / \mathrm{mL}$, Sigma, USA) and $\beta$-mercaptoethanol (0.5\%)) was added and incubated for $5 \mathrm{~min}$ at room temperature. The gliding process was observed using a fluorescent microscope with a 100x objective $(\mathrm{NA}=1.4)$. The average microtubule gliding speed was calculated from 150 individual microtubules and hybrids respectively.

For the kinesin stepping assay, first BRB80 buffer $(20 \mu \mathrm{L})$ was added into the flow cell then anti-tubulin antibody $(20 \mu \mathrm{L}, 100 \mu \mathrm{g} / \mathrm{mL}, \mathrm{BRB} 80$ buffer, $\mathrm{pH} 7.3)$ was flowed into the flow cell and incubated for $5 \mathrm{~min}$, at room temperature. Subsequently, Pluronic F-127 (20 $\mu \mathrm{L}$, 1\%, PF-127, Sigma, USA, BRB80 buffer, $\mathrm{pH} 7.3$ ) was added into the flow cell and incubated for another $5 \mathrm{~min}$ at the room temperature. Upon the incubation, kinesin-microtubules or kinesin-hybrids mixture $(20 \mu \mathrm{L}$, in BRB80 buffer which containing microtubule $(16 \mathrm{nM})$ or the equivalent concentration of the hybrids, MgATP $(1 \mathrm{mM})$, d-glucose $(20 \mathrm{mM})$, glucose oxidase $(0.02 \mathrm{mg} / \mathrm{mL})$, catalase $(0.8 \mathrm{mg} / \mathrm{mL})$ and $\beta$-mercaptoethanol $(0.5 \%)$ was also added and incubated for $5 \mathrm{~min}$ at room temperature. The kinesin stepping process onto the microtubule or hybrid was observed using fluorescence microscopy as previously described. The average kinesin stepping speed was calculated from 38 and 34 individual kinesins on the microtubules and hybrids (15 independent experiments) respectively.

\section{Statistical analysis}

All tables are presented as the average value with (+/-) SD values. Significance was determined by student T-test with $\mathrm{p}<0.05^{*}$ indicating significance. 


\section{Results and Discussion}

From natural computing or computing with natural materials ${ }^{44}$, to synthetic analog composites $^{45}$, and from bio-inspired structures for battlefield armors ${ }^{46}$ to recombinant fibers such as silk for healthcare applications ${ }^{47}$, the design and implementation of biological functions to non-biological materials created opportunities for the formation of hybrid systems with improved structural characteristics and applications ${ }^{48}$. Herein we hypothesize that biological materials conjugation to non-biological lab-synthesized structure could lead to the design of the next generation of hybrids to be used for synthetic conductive nanowires ${ }^{30}$ formation and placement. To demonstrate our hypothesis, we first synthesized regular templates of nanometer sized S-doped C-nanodots (S-doped C-dots) using a hydrothermal $\operatorname{method}^{36}$ and sulfur as a precursor ${ }^{36}$. Elemental composition of the lab synthesized S-doped C-dots was confirmed using X-ray photoelectron (XPS) and energy-dispersive X-ray (EDX) spectroscopies (Figure S1). Complementarily, transmission electron microscopy (TEM; Figure S2 a) and scanning electron microscope (SEM; Figure S2 b) confirmed the morphology of the S-doped C-dots and showed well-separated and quasi-spherical nanodots. S-doped C-dots had a rather narrow size (between 2 to $10 \mathrm{~nm}$ ) with an average of about 7.2 $\mathrm{nm}$; further, more than $94 \%$ of the nanodots analyzed showed diameters of less than $12 \mathrm{~nm}$ thus indicating controlled synthesis.

Such lab synthesized nanodots were used as nanotemplates for physical binding of free tubulin, a precursor of microtubule cytoskeletal filaments (Figure 1a). The resulting tubulin-S-doped C-dots conjugates were mixed with additional free tubulin in solution and polymerized under the chemical energy of guanosine triphosphate (GTP) to create hybrids. Control experiments using solely free tubulin assembly and the GTP energy source were also used to form microtubules in vitro.

Tubulin attachment was confirmed using spectroscopic analysis. Specifically, Figure 1 b shows the UV absorbance of the S-doped C-dots used as controls (red curve) and tubulin-S-doped C-dot conjugates (black curve) respectively. As shown, both the S-doped C-dots and the conjugates showed absorbance at $330 \mathrm{~nm}$ confirming the presence of the S-doped C-dots starting material (Table S1) ${ }^{36}$. Further, when compared to control S-doped C-dots, the conjugates displayed a change in their absorbance signal. Such change could be due to (1) tubulin attachment leading to larger structures formation, (2) spectral variations due to varying composition, and/or (3) a change in the turbidity of the solution. Indeed, for (1) previous analysis have showed that larger structures could potentially hinder the UV 
absorbance of the sample or change inter-particle distance ${ }^{49}$. In particular, it was showed that an increase in particle size could enhance Rayleigh scattering ${ }^{50}$ while reducing the adsorption $^{51}$. Indeed, control tubulin did not show such a significant change in spectra at the wavelength used for observation (Figure S3). For (2), a varying composition was shown to directly influence molecular dispersion ${ }^{52}$ that could have diminished the scattered light intensity $^{52}$. Lastly for (3), previous analysis have showed that indeed, without the effect of turbidity, the decrease in scattering would be less marked ${ }^{53}$, with a clear specimen yielding $100 \%$ transmittance, while a turbid sample will deflect a considerable portion of the light rays and will have a lower percent transmittance.

a)

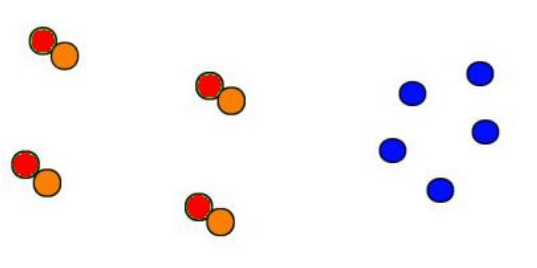

Tubulin Dimer $\quad$ S-doped C-dots

b)

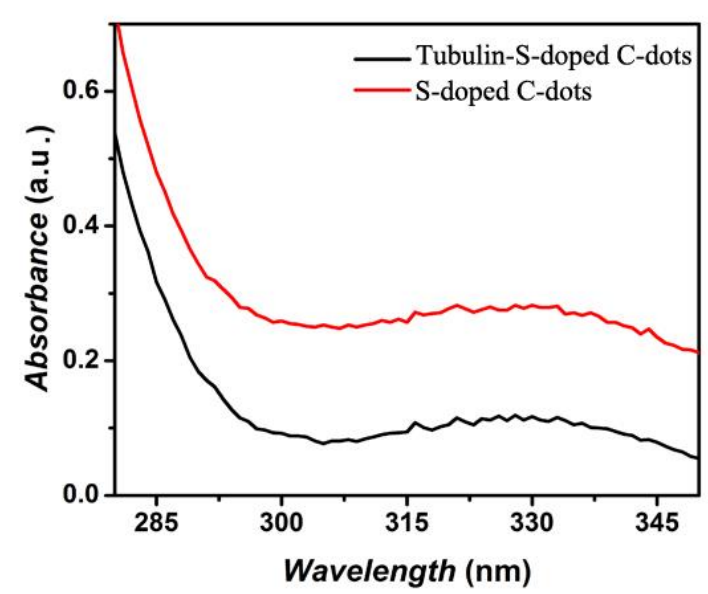

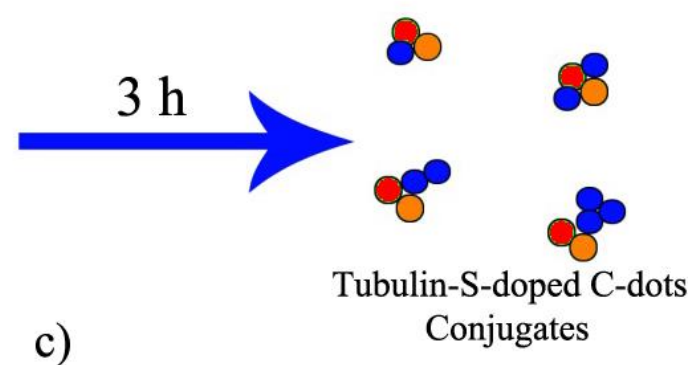

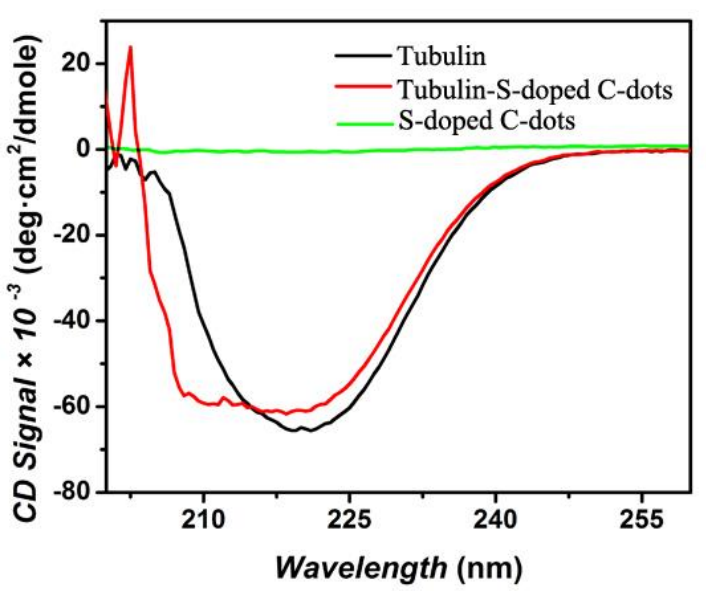

Figure 1: (a) Scheme of tubulin attachment onto user-synthesized S-doped C-nanodots (S-doped C-dots). (b) UV absorbance of S-doped C-dots and tubulin-S-doped C-dot conjugates respectively. (c) Circular dichroism (CD) graph of tubulin, S-doped C-dots and tubulin-S-doped C-dot conjugates respectively.

Templated tubulin biological functionality was tested using structure analysis performed by circular dichroism (CD). The results were evaluated against the structure of the unbound i.e., free tubulin, in solution; control of the S-doped C-dots were also included. Such analysis were performed since previous reports confirmed structural changes for human serum 
albumin and entropy-driven binding when a protein was immobilized onto polymer particles $^{54}$ or changes after insulin was entrapped into chitosan/alginate nanoparticles ${ }^{55}$. Previous studies have also showed that once proteins attach to nanosupports such as nanotubes $^{56}$, titanium dioxide nanoparticles ${ }^{57}$ or nanoclays ${ }^{58}$, their internal structural deformation could lead to loss of functionality ${ }^{59}$.

Our results showed that both tubulin and tubulin-S-doped C-dots conjugates had similar ellipticity (Figure $1 \mathbf{c}$ ), where ellipticity is the tangent of the ratio of the minor to major elliptical axis ${ }^{60}$ thus indicating very similar secondary structures for the free and immobilized tubulin respectively. A negative band appeared at $205 \sim 225 \mathrm{~nm}$ and was mainly associated with $\alpha$-helix structures of the protein ${ }^{61}$, with the band becoming minimally broader after the protein was bound to the S-doped C-dots. This change, while not significant, was presumably due to the curved nanoparticle surface (i.e., herein the S-doped C-dots, see Figure S2); previous studies have shown that particle curvature could indeed affect secondary structures of the immobilized proteins and in cases, even lead to irreversible changes of such structures $^{62}$. These results are in contrast with previous studies that have showed for instance, that the random coils connecting the $\alpha$-helices in the human serum albumin were strongly affected after protein binding to nanotubes ${ }^{63}$, with irreversible changes occurring both in the stability of the protein as well as in its secondary structure. Similar results were also recorded after desorbing this protein from polystyrene particles ${ }^{64}$. Complementarily, Bellezza et al., ${ }^{65}$ suggested that the interaction of myoglobin with phosphate and phosphonate grafted-zirconia nanoparticles induced the loss of the secondary structure ( $\alpha$-helices) in the myoglobin. Further, our analysis are also contrast with previous research that showed tubulin structure alteration upon binding to fullerene derivative or ferric oxide nanoparticles ${ }^{66}$.

Our analysis using Circular Dichroism Neural Networks (CDNN) $\operatorname{method}^{67}$ also confirmed the minimal changes in the immobilized protein (Table 1). Specifically, the $\alpha$-helix content was shown to increase (from $37.1 \%$ to $43.6 \%$ ), while $\beta$-sheets and random coil contents were shown to decrease (from $30.8 \%$ to $27.8 \%$, and from $31.3 \%$ to $26.8 \%$ respectively) after tubulin immobilization onto the S-doped C-dots. Such changes, once again, while not statistically relevant, could be a reflection of small conformational rearrangements which resulted from the tubulin attachment ${ }^{62}$. In particular, such differences could be presumably due to the either/or association and dissociation rates that each attached tubulin is undergoing at the surface of a given nanodot. Such rates could dictate the longevity of their individual interactions to be governed by the particle geometry and size, and its surface 
curvature $^{68}$. Previous reports have showed that proteins and particles of the same scale could still undergo different conformational changes with such changes however not affecting protein overall functionality, i.e. when compared to that of the starting molecule, as it was the case for the human carbonic anhydrase $(29 \mathrm{kDa})$ when bound to $\mathrm{SiO}_{2} \mathrm{NPs}(\mathrm{d}=6,9,15 \mathrm{~nm})^{69}$.

Table 1: CD characterization of tubulin protein and tubulin-S-doped C-dot conjugates.

\begin{tabular}{|l|l|l|l|l|l|l|}
\hline Protein & $\begin{array}{l}\text { Helix } \\
(\boldsymbol{\%})\end{array}$ & $\begin{array}{l}\text { Antipar } \\
\text { allel }(\boldsymbol{\%})\end{array}$ & $\begin{array}{l}\text { Paralle } \\
\mathbf{l}(\boldsymbol{\%})\end{array}$ & $\begin{array}{l}\text { Beta-Tur } \\
\mathbf{n}(\boldsymbol{\%})\end{array}$ & $\begin{array}{l}\text { Random } \\
\text { Coil }(\boldsymbol{\%})\end{array}$ & $\begin{array}{l}\text { Total Sum } \\
(\boldsymbol{\%})\end{array}$ \\
\hline Tubulin & $37.1 \pm 0.9$ & $7.0 \pm 0.4$ & $8.0 \pm 0.4$ & $15.8 \pm 0.3$ & $31.3 \pm 0.6$ & $99.2 \pm 0.5$ \\
\hline $\begin{array}{l}\text { Tubulin-S-dop } \\
\text { ed C-dots }\end{array}$ & $43.6 \pm 0.6$ & $6.0 \pm 0.2$ & $6.6 \pm 0.3$ & $15.1 \pm 0.7$ & $26.8 \pm 0.8$ & $98.1 \pm 0.8$ \\
\hline
\end{tabular}

Considering that the immobilized tubulin protein highly retained its secondary structures, we further evaluated its activity. Herein tubulin's activity is defined as the ability of the immobilized protein to polymerize when interfaced with other free tubulin in solution, all in the presence of guanosine triphosphate (GTP) chemical energy. Such polymerization was previously observed both in vivo and in vitro for free tubulin in solution and was shown to lead to the synthesis of the microtubule cytoskeletal filament ${ }^{43}$. The activity was to be tested since previous reports have showed impediments of the release of inorganic phosphate in GTP hydrolysis ${ }^{70}$ and inhibition of tubulin polymerization ${ }^{71}$ upon protein immobilization. 
a)

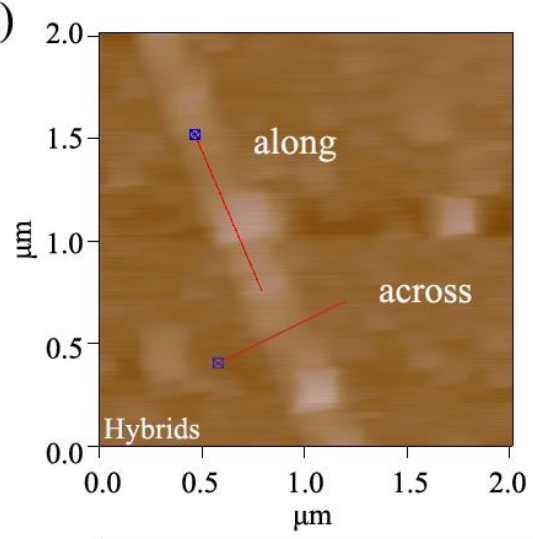

c)

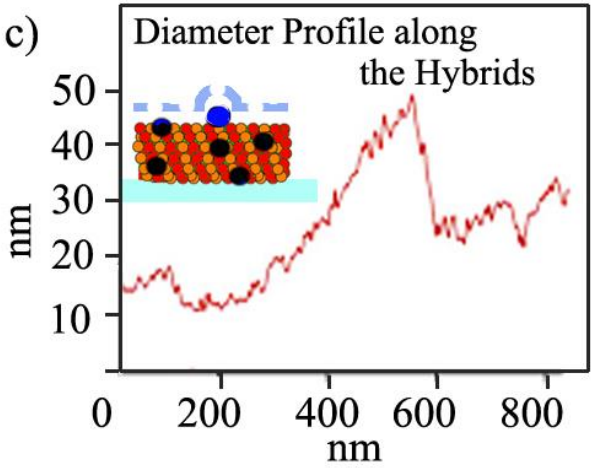

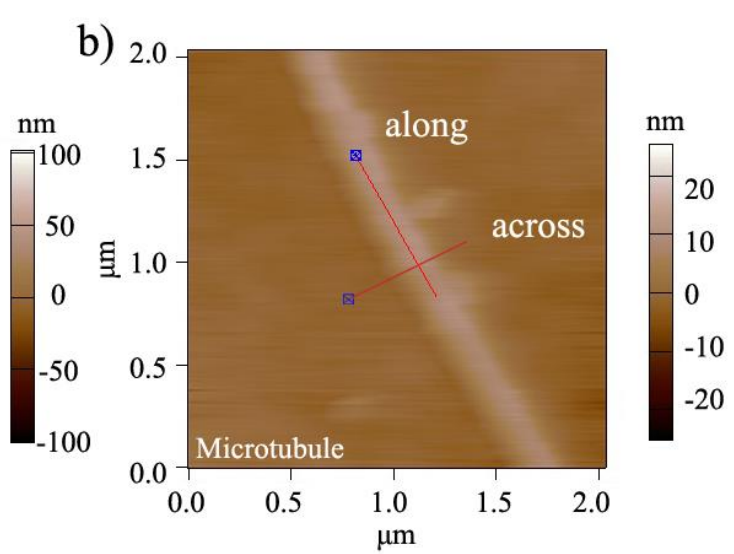

d) 50

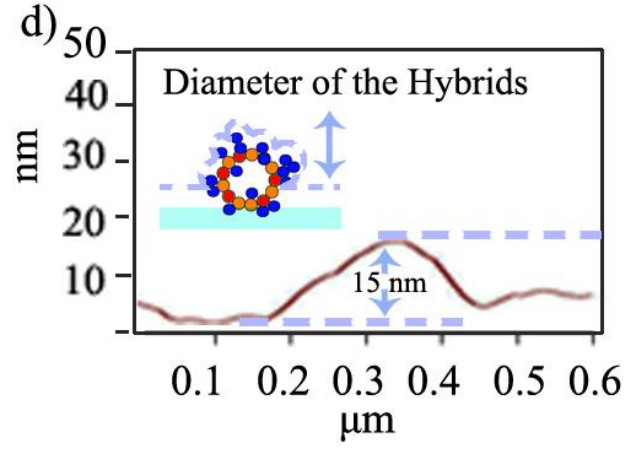

e)

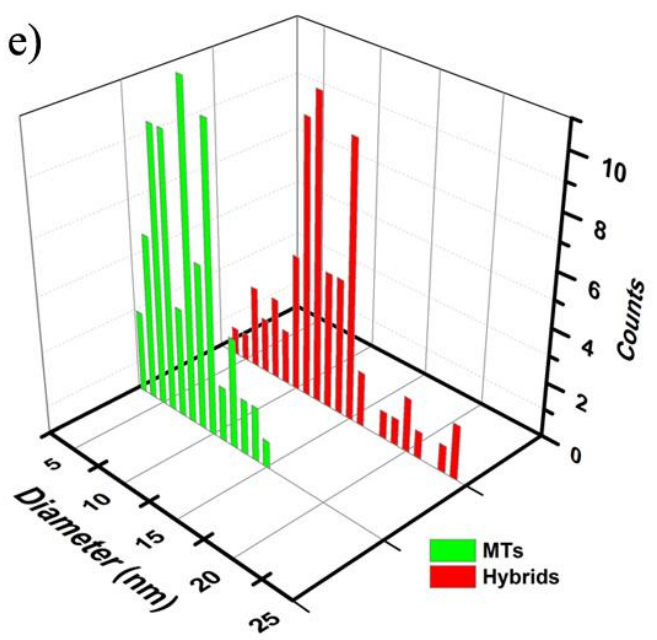

f)

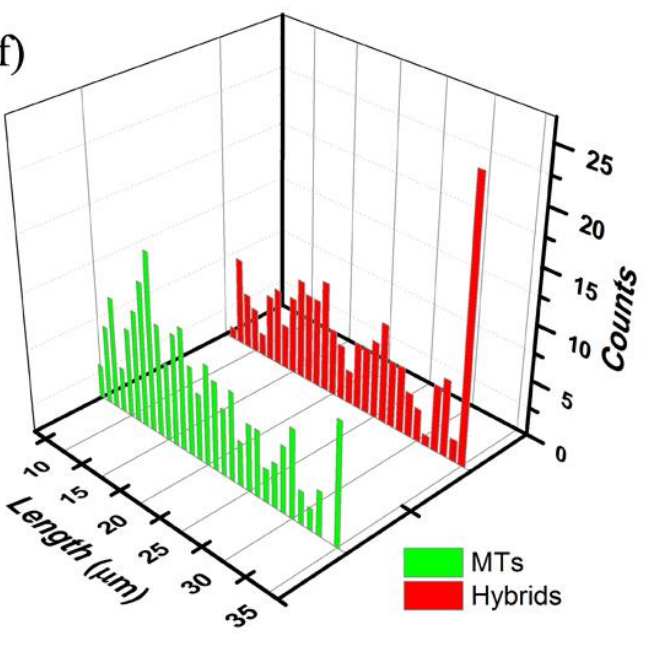

Figure 2: (a) Representative atomic force microscopy-based (AFM) morphology of S-doped C-dot hybrid after crosslinking with glutaraldehyde. (b) Representative morphology AFM analyses of microtubule after crosslinking with glutaradehyde (control). Schematic representation of the AFM height distribution analysis along (c) and across (d) a hybrid. (e) Diameter distributions of 83 individual microtubules and 68 hybrids collected from 25 and 20 independent images respectively. (f) Height distributions of 211 individual microtubules and 181 hybrids collected from 25 and 20 independent videos respectively. 
Results showed that when free tubulin was incubated with the conjugates under the GTP energy, microtubule-like morphologies were achieved. Such linear morphologies of the resulting structures, also identified as hybrids (Figure 2a), were compared to those of the control linear microtubules (Figure 2b) obtained when free tubulin polymerized in the presence of GTP. Atomic force microscopy (AFM) analysis of diameter of these two structures along and across showed variations consistent with the presence of the S-doped C-dots. In particular, analysis performed along the hybrids or microtubules (red lines in the individual images) showed height variations from 10 to $48 \mathrm{~nm}$ (Figure 2c) for hybrid and from 7 to $10 \mathrm{~nm}$ for microtubules used as controls (Figure S4a). Complementary, height analysis performed across the two structures also showed variations from 11 to $17 \mathrm{~nm}$ for hybrids and from 3 to $12 \mathrm{~nm}$ for microtubules used as controls (Figure S4b). Previous study has showed such height variations could be presumably due to the uneven distribution of the glutaraldehyde or the mica surface-coating agent used to ensure specific attachment of microtubule or hybrid for AFM analysis or the uneven distribution of the S-doped C-dots into the hybrid itself $^{30,72}$. Indeed, for the hybrids, there were also dots-like structures being observed in the recorded AFM images to confirm the successful entrapment of the S-doped C-dots. Figure 2e compares the diameter while Figure $2 \mathbf{f}$ compares the length distributions of microtubules and hybrids for significant statistics. The variations in the diameter supports previous reports ${ }^{72}$ while the similarities in controlled assembly, geometries and length demonstrate the feasibility and ease of the method create hybrid structures of controlled aspect ratios and surface characteristics.

Upon demonstrating the controlled assembly of the hybrids, we further evaluated their functionality, herein defined as the ability of kinesin 1 molecular motor (simply called kinesin) to recognize their linearity and attach to their structure. In the cell, microtubules are being recognized by kinesin and used as tracks for the transport of organelles ${ }^{73}$ or synaptic vesicle precursor $^{74}$ with kinesin walking on individual microtubule protofilaments in a processive manner by transforming the chemical energy of adenosine triphosphate (ATP) into mechanical work $^{43}$. Kinesin ability for recognition and binding was tested under adenylyl-imidodiphosphate (AMP-PNP) energy (Figure 3a; AMP-PNP is known as a stable form of ATP being able to hydrolyzable extremely slowly ${ }^{75}$ ) and using AFM analysis. Analysis of the kinesin bound to the hybrids or kinesin bound to the control microtubules are shown in Figure 3b and $\mathbf{3 c}$ respectively. As shown, the linearity of the hybrid or control microtubule was conserved even upon binding; images analysis identified the bound kinesins 
as dots-like geometries onto the individual samples.

Height distribution AFM analysis performed across the hybrid or microtubule (along the red line identified in the above figures) showed that the bound kinesin had a height distribution from 5 to $12 \mathrm{~nm}$ when interfaced with the hybrids and from 2 to $7 \mathrm{~nm}$ when interfaced with the microtubules. Figure 3d and 3e compare the diameter distributions between kinesin-hybrid and kinesin-microtubule respectively for a large set of samples. Summary of the height distribution profiles shows values in $\mathrm{nm}$ scale, consistent with previous results ${ }^{76}$. The height variations observed for the immobilized kinesins in our experiments when compared to previous study (i.e., variation from the reported $3 \mathrm{~nm}^{76}$ to 5 to $12 \mathrm{~nm}$ for the hybrids or 2 to $7 \mathrm{~nm}$ for the microtubule, in this study) is attributed to kinesin's possible orientation onto the surface of the hybrid or microtubule respectively (Figure 3f). Specifically, previous reports showed that when interfaced with microtubules in vitro, standard orientation, in which the core $\beta$ sheet is face-on while helices $\alpha 1-\alpha 3$ are in front of the sheet and $\alpha 4-\alpha 6$ are in the back, could be observed. A "V shape orientation" that rotates the standard view to $65^{\circ}$ about the vertical axis was also found ${ }^{77}$. Further, such height variation could also be a result of kinesin laying on different filaments of the imaged structure since previous reports have showed that kinesin binds to different protofilaments without selectivity ${ }^{78}$. Indeed, our control experiments have shown that single kinesin molecules immobilized onto mica surfaces (Figure S5a) and analyzed using contact mode had diameter variations between 2-12 $\mathrm{nm}$ (Figure S5b). 
a)

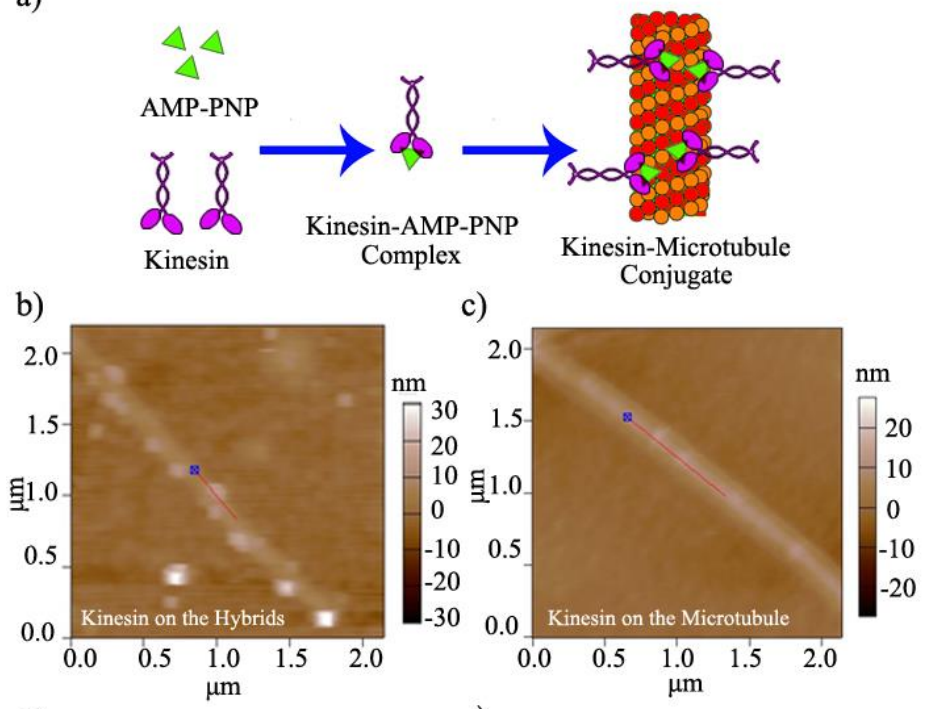

d)

e)
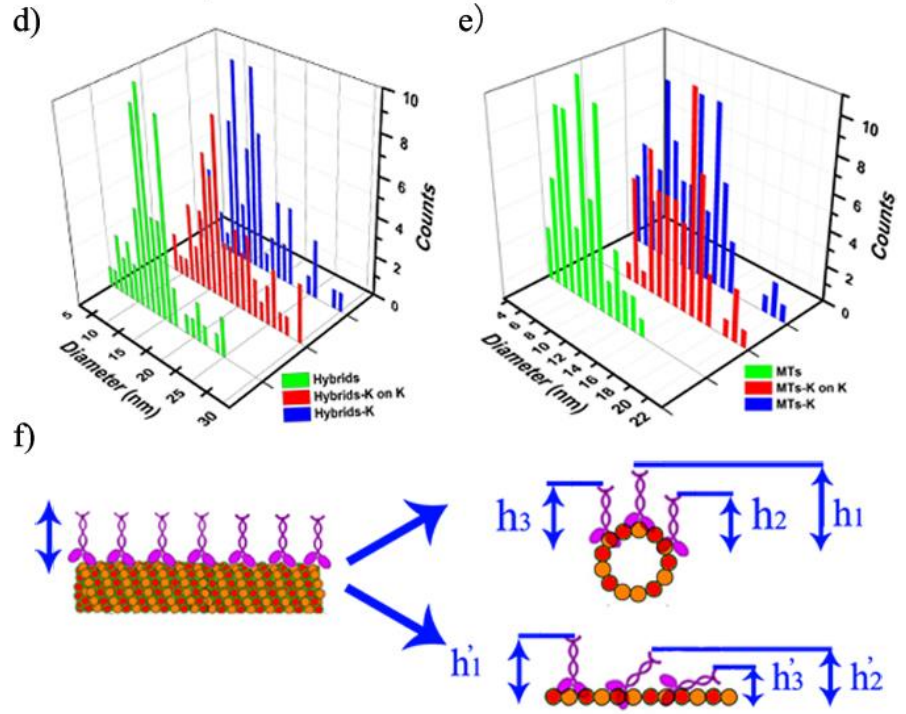

Figure 3: (a) Scheme of the kinesin-microtubule/hybrid binding in the presence of AMP-PNP. Representative AFM images of kinesin-hybrid (b) and kinesin-microtubule used as control (c). The line was chosen to follow the morphology of the sample for height analysis. d) The diameter distribution of 68 individual hybrids, 66 individual kinesin-hybrids measured on the kinesin and 80 individual kinesin-hybrid measured randomly, all collected from 20 and 22 independent images respectively. e) The diameter distribution of 83 individual microtubules, 70 individual kinesin-microtubule measured on the kinesin dot itself and 82 individual kinesin-microtubule measured randomly and collected from 25 and 23 independent images respectively. (f) Possible orientations of kinesin onto microtubule/hybrid. 


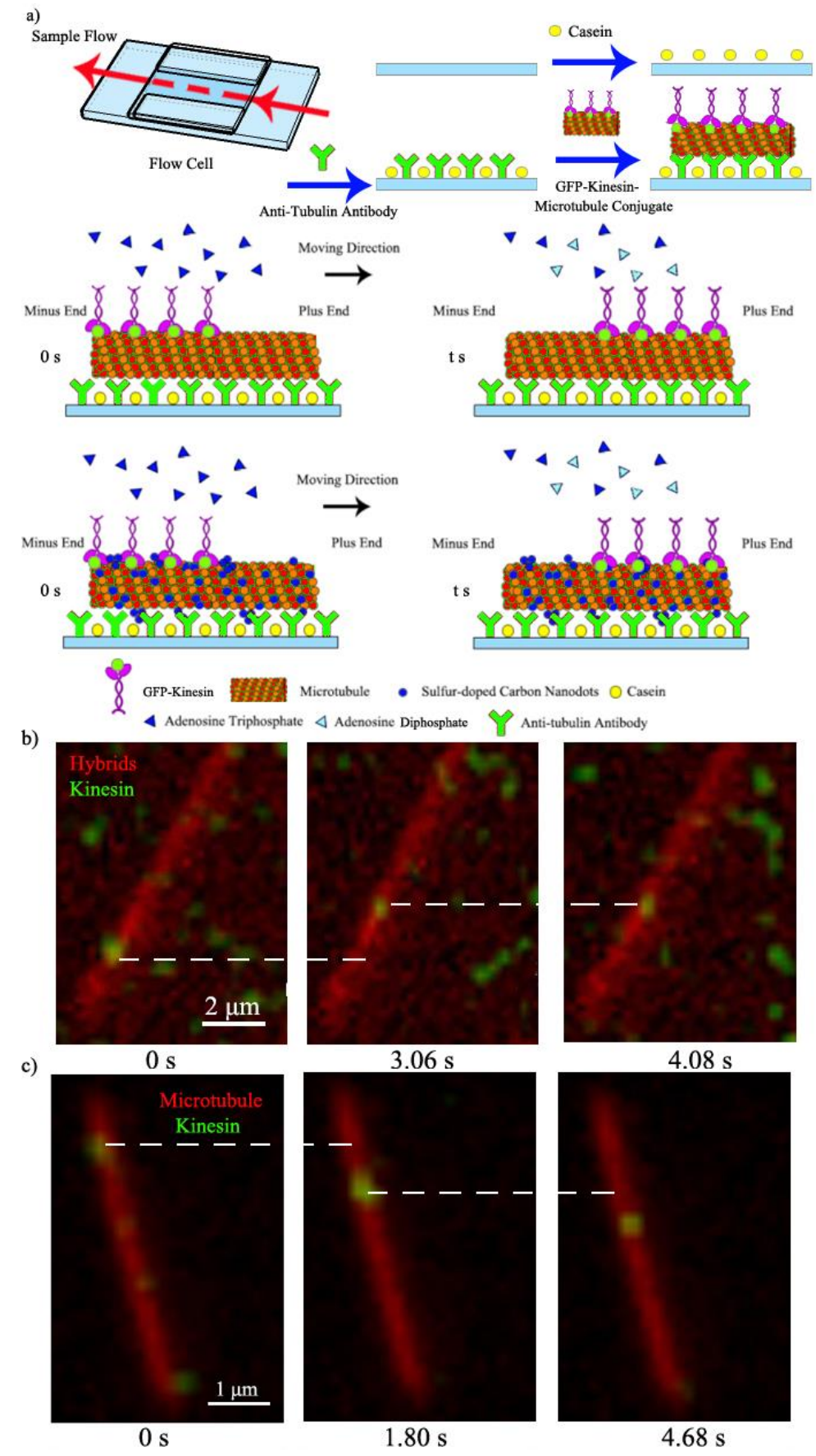

Figure 4: (a) Schematic of the stepping assay. Briefly, a user-made flow cell was coated with anti-tubulin antibody for protein specific interactions. The stepping activity of the kinesin was observed using fluorescent microscopy, all under provided chemical ATP energy. (b) Fluorescent images of kinesin-hybrids at different time points; analysis (white line) show kinesin (green) ability to step on hybrids (red) as shown by the distance displacement. (c) Fluorescent images of kinesin-microtubule at different time (controls); again, analysis (white line) show kinesin (green) ability to step on control microtubule (red) as recorded by distance displacement. 
The ability of the hybrids to perform tasks in synthetic environments using the templated kinesin as a precursor was tested using established stepping and gliding assays. In the stepping assay, kinesin is walking towards the plus end (or fast growing end) of a surface immobilized microtubule by mimicking its cellular function of cargo transport ${ }^{79}$. Such ability was previously demonstrated to be used in vitro to transport synthetic metals or semiconductor nanoparticle cargos ${ }^{80}$. In the gliding assay, surface immobilized kinesin are gliding microtubules in vitro to transport micrometer-sized cargoes across different arrays or selective load of biosensing molecules ${ }^{81}$.

Figure 4a shows the schematic of the proposed stepping assay, in which kinesin-hybrids or kinesin-microtubules are introduced into a user-made flow cell, with casein used as coating protein to limit non-specific binding and loss of protein activity ${ }^{82}$. Anti-tubulin antibodies were used as site-specific forms of interaction (i.e., between templated tubulin and anti-tubulin antibodies respectively) of the hybrid or the microtubule control. Our analysis showed that kinesin molecules were not only able to recognize the structure of the hybrids (as identified by the superposition of green-kinesin and red-microtubule signals in the optical microscopy), but further, they were also able to move onto such structure when the energy source in the environment was exchanged from AMP-PNP to $\mathrm{ATP}^{83}$. The average kinesin speeds onto hybrids (Figure 4b) or microtubules (Figure 4c) were 0.3 or $0.2 \mu \mathrm{m} / \mathrm{s}$ respectively (results collected from 15 independent experiments). In addition, kinesins' dissociation from the hybrids or microtubule was identified as a disappearance of the green signal in the fluorescence timeframe. Such results confirm the ability of the hybrids to be serving as templates for kinesin, with kinesin movement being in a similar manner to that onto the control microtubules ${ }^{84}$. While previous reports identified that kinesin could recognize control microtubules to perform processive transport of nanocrystal ${ }^{85}$ and metal nanoparticles $^{80}$, to our knowledge this is the first demonstration of kinesin to recognize non-biological hybrid structures and move in a processive manner and at a similar speed as for the control microtubule.

Complementary, in the gliding assay (Figure 5a) kinesin was again not only able to recognize the structure of the hybrid (Figure 5b) but also able to glide it onto surfaces when the energy source was exchanged from AMP-PNP to ATP ${ }^{83}$. Optical imaging analysis also revealed that all of microtubules (Figure 5c) or hybrids in the field of view moved in random directions, which was previously shown to be related to the orientation of the microtubule and/or kinesin onto the substrate ${ }^{86}$. The average speed of microtubules was about $0.49 \pm 0.12$ 
$\mu \mathrm{m} / \mathrm{s}$ (150 microtubules evaluated), while that of the gliding hybrids was slightly higher however not significant (i.e., $0.52 \pm 0.14 \mu \mathrm{m} / \mathrm{s}$; again, 150 hybrids were evaluated; Supporting Movie S1 and S2). Our analysis of a similar gliding velocity of the hybrid and microtubule used as control are supported by previous studies which showed that for both, single- and multi-motor in vitro motility assays, incorporation of additional functionality into the microtubule does not affect kinesin velocity and run length ${ }^{87}$. Moreover, previous research has also showed that if kinesin is encountering an obstacle on the microtubule structure, it could switch protofilaments and still be able to perform gliding in synthetic environment with the off-axis steps not making a large contribution to the motor protein velocity on decorated tracks ${ }^{88}$. 
a)

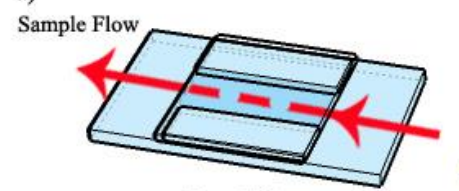

Flow Cell
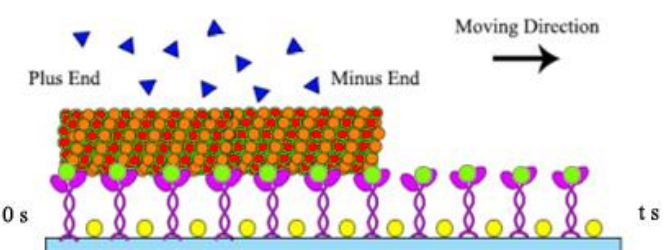

Moving Direction

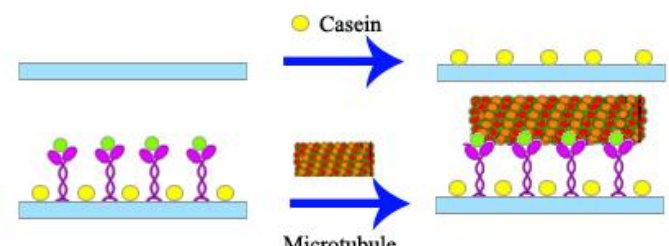

Microtubule

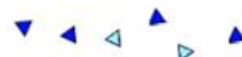

Plus End $\nabla \longrightarrow \triangleright$ Minus End
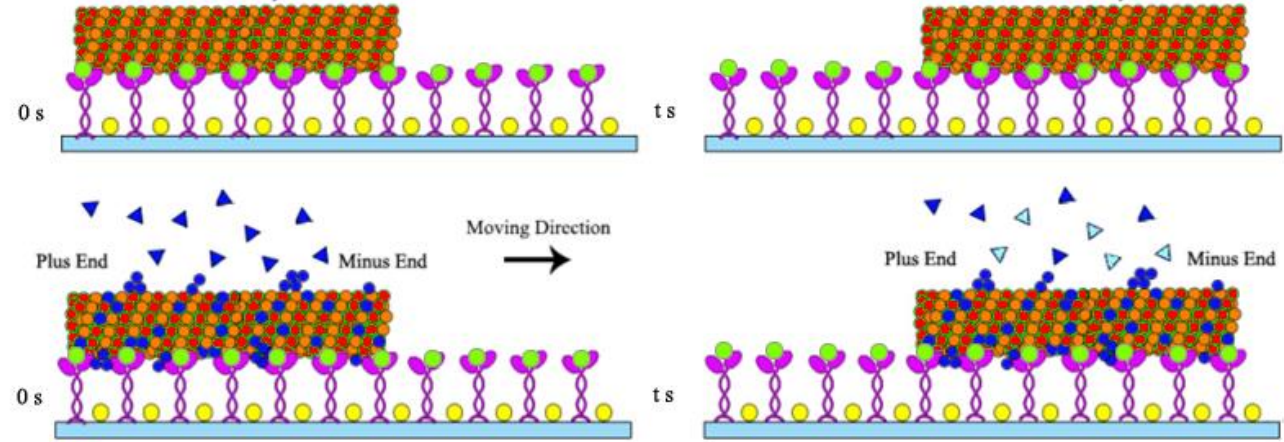

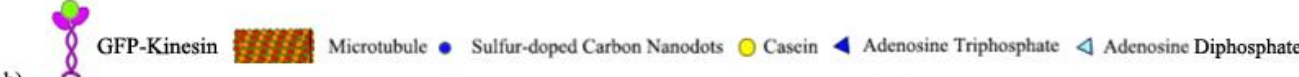
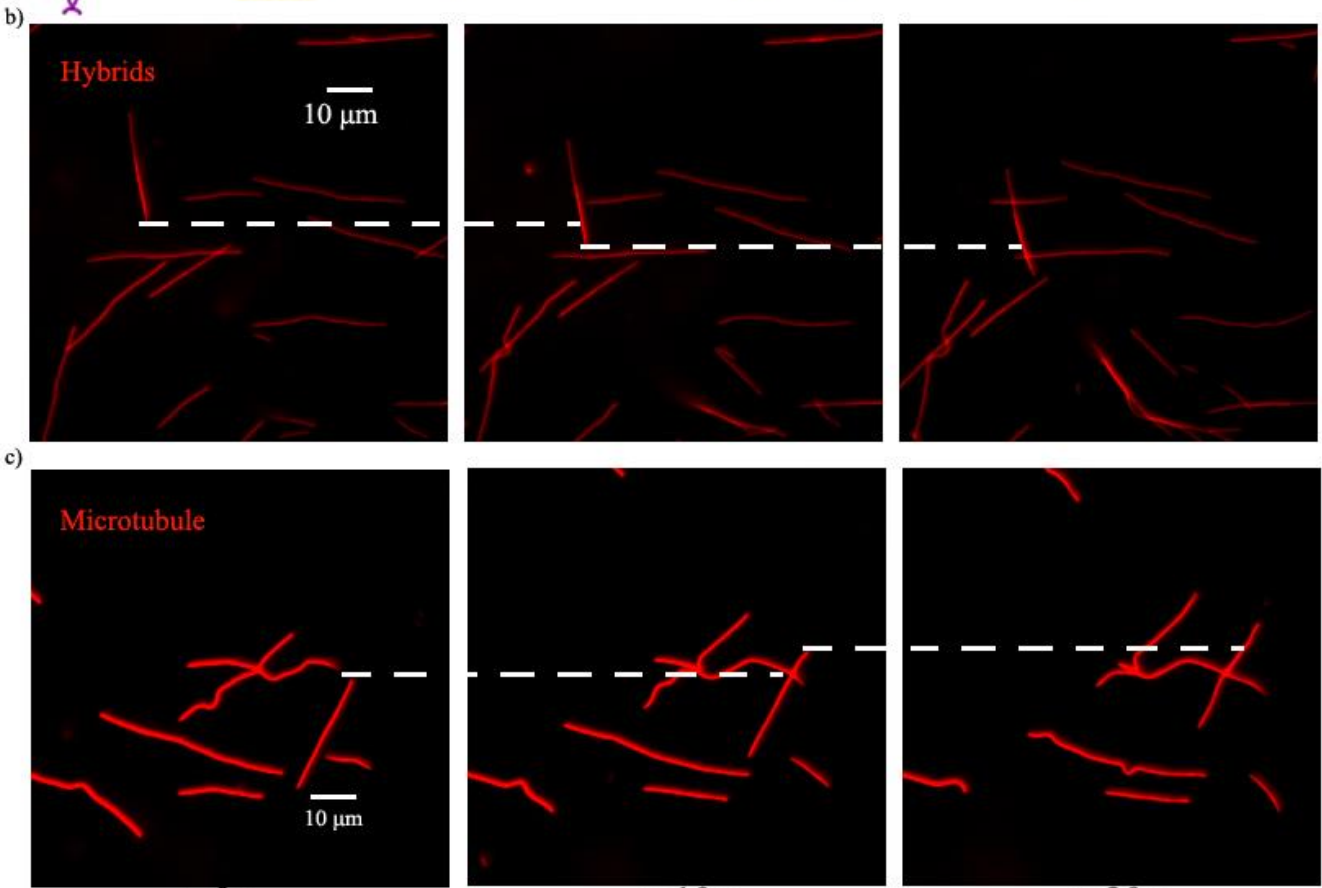

$\mathrm{t}=0 \mathrm{~s}$

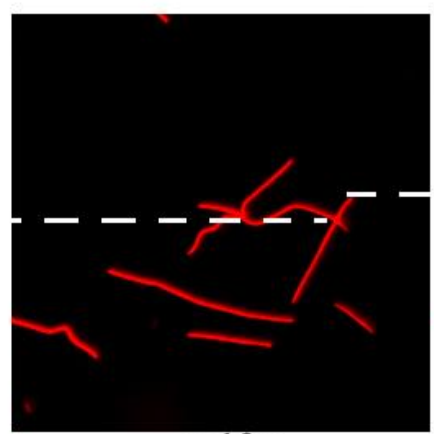

$\mathrm{t}=10 \mathrm{~s}$

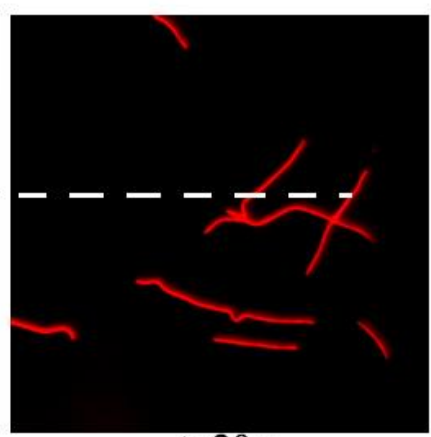

$\mathrm{t}=20 \mathrm{~s}$

Figure 5: (a) Schematic of the gliding assay; casein was used as a blocking molecule to prevent non-specific binding of microtubules or hybrids. AMP-PNP bound GFP-kinesin attached to the flow cell was used to propel microtubules or hybrids when the AMP-PNP energy source was exchanged for ATP. (b) Fluorescent images of hybrids glided onto surface immobilized kinesin. (c) Fluorescent images of microtubules glided onto surface immobilized kinesin. 
Once again, to our knowledge, this is the first demonstration of hybrid movement on kinesin functional surfaces. Moreover, to our knowledge, this is the first demonstration of integrated self-assembly and self-recognition biological functions in a hybrid formation that possesses sensing ability, i.e. in which the sensing is defined as both by the ability of the kinesin to recognize and move onto such structure as well as by the photoluminescence of the C-dots which were shown to be used in applications of $\mathrm{Fe}(\mathrm{III})$ detection ${ }^{36}$ for instance. Beside a viable demonstration of biological function implementation (i.e., implementing tubulin-related functions to a non-biological material, S-doped C-dots), such functionality also benefits of the known stability of the hybrids ${ }^{30}$. Specifically, previous studies have showed that such hybrids are stable for up to 9 days while at room temperature which is in contrast with maximum 5 days recorded for the microtubules ${ }^{30}$. It is thus envisioned that when one combines the stability of the hybrids with the functionality of kinesin and its ability to recognize and perform tasks on synthetic materials, individual molecular transport means to specific location could be achieved to benefit of electrical conductivity at nanointerfaces for biosensing ${ }^{81}$ or molecular sorting ${ }^{89}$. 


\section{Conclusions}

Our experiments have demonstrated activity and functionality of a hybrid material created from tubulin and S-doped C-dots. In particular, our experiments have demonstrated tight synthesis of nanodots to be used for attachment of biologically functional molecules of tubulin. The self-assembly properties of such molecules were further used to support the synthesis of hybrid structures, i.e. microtubules incorporating S-doped C-dots. The hybrids were manipulated in synthetic environment using kinesin-microtubule like functionality. Our experiments have the ability to be used for designing the next generation of sensors with high ability for detection (if one kinesin motor is used) or for load transport under ATP energy and the stability of non-biological material used as template. 


\section{References:}

1 Hurst, S. J., Payne, E. K., Qin, L. \& Mirkin, C. A. Multisegmented one - dimensional nanorods prepared by hard - template synthetic methods. Angewandte Chemie International Edition 45, 2672-2692 (2006).

2 Pan, H. et al. Growth of single-crystalline Ni and Co nanowires via electrochemical deposition and their magnetic properties. The Journal of Physical Chemistry B 109, 3094-3098 (2005).

3 Xiang, B. et al. Rational synthesis of p-type zinc oxide nanowire arrays using simple chemical vapor deposition. Nano Letters 7, 323-328 (2007).

4 Sunkara, M. K., Sharma, S., Miranda, R., Lian, G. \& Dickey, E. Bulk synthesis of silicon nanowires using a low-temperature vapor-liquid-solid method. Applied Physics Letters 79, 1546-1548 (2001).

5 McAlpine, M. C. et al. High-performance nanowire electronics and photonics on glass and plastic substrates. Nano Letters 3, 1531-1535 (2003).

6 Zheng, G., Patolsky, F., Cui, Y., Wang, W. U. \& Lieber, C. M. Multiplexed electrical detection of cancer markers with nanowire sensor arrays. Nature biotechnology $\mathbf{2 3}$, 1294-1301 (2005).

7 Farsad, M., Vernerey, F. J. \& Park, H. S. An extended finite element/level set method to study surface effects on the mechanical behavior and properties of nanomaterials. International Journal for Numerical Methods in Engineering 84, 1466-1489 (2010).

8 Thelander, C. et al. Nanowire-based one-dimensional electronics. Materials today 9, 28-35 (2006).

9 Hiruma, K., Murakoshi, H., Yazawa, M. \& Katsuyama, T. Self-organized growth of GaAsInAs heterostructure nanocylinders by organometallic vapor phase epitaxy. Journal of Crystal Growth 163, 226-231 (1996).

10 Xiang, D. Fabrication and utilization of mechanically controllable break junction for bioelectronics. (Forschungszentrum Jülich, 2012).

11 Cardoza-Contreras, M. N. et al. Single ZnO nanowire-based gas sensors to detect low concentrations of hydrogen. Sensors 15, 30539-30544 (2015).

12 Liao, L. et al. High-speed graphene transistors with a self-aligned nanowire gate. Nature 467, 305-308 (2010).

13 Avouris, P., Appenzeller, J., Martel, R. \& Wind, S. J. Carbon nanotube electronics. Proceedings of the IEEE 91, 1772-1784 (2003). 
14 LeRoy, B. J., Lemay, S., Kong, J. \& Dekker, C. Electrical generation and absorption of phonons in carbon nanotubes. Nature 432, 371-374 (2004).

15 Kymakis, E. \& Amaratunga, G. Single-wall carbon nanotube/conjugated polymer photovoltaic devices. Applied Physics Letters 80, 112-114 (2002).

16 Ming, H. et al. Large scale electrochemical synthesis of high quality carbon nanodots and their photocatalytic property. Dalton Transactions 41, 9526-9531 (2012).

17 Baker, S. N. \& Baker, G. A. Luminescent carbon nanodots: emergent nanolights. Angewandte Chemie International Edition 49, 6726-6744 (2010).

18 Mao, L.-H. et al. Facile access to white fluorescent carbon dots toward light-emitting devices. Industrial \& Engineering Chemistry Research 53, 6417-6425 (2014).

19 Liu, C. et al. One-step synthesis of surface passivated carbon nanodots by microwave assisted pyrolysis for enhanced multicolor photoluminescence and bioimaging. Journal of Materials Chemistry 21, 13163-13167 (2011).

20 Qu, S., Chen, H., Zheng, X., Cao, J. \& Liu, X. Ratiometric fluorescent nanosensor based on water soluble carbon nanodots with multiple sensing capacities. Nanoscale $\mathbf{5}$, 5514-5518 (2013).

21 Zhang, Y.-Q. et al. One-pot synthesis of N-doped carbon dots with tunable luminescence properties. Journal of Materials Chemistry 22, 16714-16718 (2012).

22 Shi, W. et al. Carbon nanodots as peroxidase mimetics and their applications to glucose detection. Chemical Communications 47, 6695-6697 (2011).

23 Zhou, L., Lin, Y., Huang, Z., Ren, J. \& Qu, X. Carbon nanodots as fluorescence probes for rapid, sensitive, and label-free detection of $\mathrm{Hg} 2+$ and biothiols in complex matrices. Chemical Communications 48, 1147-1149, doi:10.1039/C2CC16791C (2012).

24 Cao, L. et al. Carbon nanoparticles as visible-light photocatalysts for efficient $\mathrm{CO} 2$ conversion and beyond. Journal of the American Chemical Society 133, 4754-4757, doi:10.1021/ja200804h (2011).

25 Wang, Y., Wang, X., Antonietti, M. \& Zhang, Y. Facile One - Pot Synthesis of Nanoporous Carbon Nitride Solids by Using Soft Templates. ChemSusChem 3, 435-439 (2010).

26 Li, D., McCann, J. T., Xia, Y. \& Marquez, M. Electrospinning: a simple and versatile technique for producing ceramic nanofibers and nanotubes. Journal of the American Ceramic Society 89, 1861-1869 (2006). 
27 Garreau, A. \& Duvail, J. L. Recent Advances in Optically Active Polymer - Based Nanowires and Nanotubes. Advanced Optical Materials 2, 1122-1140 (2014).

28 Kamkar, D. A., Wang, M., Wudl, F. \& Nguyen, T.-Q. Single nanowire OPV properties of a fullerene-capped P3HT dyad investigated using conductive and photoconductive AFM. ACS nano 6, 1149-1157 (2012).

29 Yan, Z. et al. Three-dimensional optical trapping and manipulation of single silver nanowires. Nano letters 12, 5155-5161 (2012).

$30 \mathrm{Hu}, \mathrm{X}$., Dong, C., Su, R., Xu, Q. \& Dinu, C. Z. Protein self-assembly onto nanodots leads to formation of conductive bio-based hybrids. 6, 38252, doi:10.1038/srep38252(2016).

31 Janke, C. \& Bulinski, J. C. Post-translational regulation of the microtubule cytoskeleton: mechanisms and functions. Nature reviews Molecular cell biology $\mathbf{1 2}$, 773-786 (2011).

32 Royle, S. J. Super-duper resolution imaging of mitotic microtubules. Nature Reviews Molecular Cell Biology 16 (2015).

33 Lehman, T. A., Smertenko, A. \& Sanguinet, K. A. Auxin, microtubules, and vesicle trafficking: conspirators behind the cell wall. Journal of Experimental Botany (2017).

34 Poruchynsky, M. S. et al. Microtubule-targeting agents augment the toxicity of DNA-damaging agents by disrupting intracellular trafficking of DNA repair proteins. Proceedings of the National Academy of Sciences 112, 1571-1576 (2015).

35 Roth, Z., Shi, L. \& Smith, D. Calcyon Regulates Axonal Transport of PI4K11a Vesicles in Cultured Rat Sensory Neurons. The FASEB Journal 29, 727.723 (2015).

$36 \mathrm{Xu}, \mathrm{Q}$. et al. Preparation of highly photoluminescent sulfur-doped carbon dots for Fe (III) detection. Journal of Materials Chemistry A 3, 542-546 (2015).

37 Yarimaga, O., Jaworski, J., Yoon, B. \& Kim, J.-M. Polydiacetylenes: supramolecular smart materials with a structural hierarchy for sensing, imaging and display applications. Chemical Communications 48, 2469-2485 (2012).

38 Levi, D. S., Kusnezov, N. \& Carman, G. P. Smart materials applications for pediatric cardiovascular devices. Pediatric research 63, 552-558 (2008).

39 Roy, I. \& Gupta, M. N. Smart polymeric materials: emerging biochemical applications. Chemistry \& biology 10, 1161-1171 (2003).

40 Gibson, D. G. et al. Enzymatic assembly of DNA molecules up to several hundred kilobases. Nature methods 6, 343-345 (2009). 
41 Campbell, A. S. et al. A Systematic Study of the Catalytic Behavior at EnzymeMetal-Oxide Nanointerfaces. Nano LIFE 4, 1450005 (2014).

$42 \mathrm{Hu}, \mathrm{X} . \&$ Dinu, C. Z. Analysis of affinities between specific biological ligands using atomic force microscopy. Analyst 140, 8118-8126, doi:10.1039/C5AN01748C (2015).

43 Dinu, C. Z. et al. Parallel Manipulation of Bifunctional DNA Molecules on Structured Surfaces Using Kinesin - Driven Microtubules. Small 2, 1090-1098 (2006).

44 Kuzum, D., Jeyasingh, R. G., Lee, B. \& Wong, H.-S. P. Nanoelectronic programmable synapses based on phase change materials for brain-inspired computing. Nano letters 12, 2179-2186 (2011).

45 Sihvola, A. Enabling optical analog computing with metamaterials. Science 343, 144-145 (2014).

46 Ehrlich, H. in Biological Materials of Marine Origin 237-262 (Springer, 2015).

47 Elices, M. et al. Bioinspired fibers follow the track of natural spider silk. Macromolecules 44, 1166-1176 (2011).

48 Wegst, U. G., Bai, H., Saiz, E., Tomsia, A. P. \& Ritchie, R. O. Bioinspired structural materials. Nature materials 14, 23-36 (2015).

49 Ashjari, M., Dehfuly, S., Fatehi, D., Shabani, R. \& Koruji, M. Efficient functionalization of gold nanoparticles using cysteine conjugated protoporphyrin IX for singlet oxygen production in vitro. RSC Advances 5, 104621-104628 (2015).

50 Mobile Phase, U. Optimizing and Monitoring Solvent Quality for UV-Vis Absorption, Fluorescence and Charged Aerosol Detectors.

51 Aaryasomayajula, V. S. R. et al. Assembly of a dual aptamer gold nanoparticle conjugate ensemble in the specific detection of thrombin when coupled with dynamic light scattering spectroscopy. Journal of Nanomedicine \& Nanotechnology 5, 1 (2014).

52 Balch, R. Measurement of turbidity with a spectrophotometer. Industrial \& Engineering Chemistry Analytical Edition 3, 124-127 (1931).

53 Parker, T. \& Dalgleish, D. The use of light - scattering and turbidity measurements to study the kinetics of extensively aggregating proteins: $\alpha$ s - Casein. Biopolymers 16, 2533-2547 (1977).

54 Lynch, I. \& Dawson, K. A. Protein-nanoparticle interactions. Nano Today 3, 40-47 (2008).

55 Sarmento, B., Ferreira, D. C., Jorgensen, L. \& van de Weert, M. Probing insulin's 
secondary structure after entrapment into alginate/chitosan nanoparticles. European Journal of Pharmaceutics and Biopharmaceutics 65, 10-17 (2007).

56 Keten, S. \& Buehler, M. J. Large deformation and fracture mechanics of a beta-helical protein nanotube: Atomistic and continuum modeling. Computer Methods in Applied Mechanics and Engineering 197, 3203-3214 (2008).

57 Xu, Z., Liu, X.-W., Ma, Y.-S. \& Gao, H.-W. Interaction of nano-TiO2 with lysozyme: insights into the enzyme toxicity of nanosized particles. Environmental Science and Pollution Research 17, 798-806 (2010).

58 Huang, X. \& Netravali, A. Characterization of flax fiber reinforced soy protein resin based green composites modified with nano-clay particles. Composites Science and Technology 67, 2005-2014 (2007).

59 Turci, F. et al. An integrated approach to the study of the interaction between proteins and nanoparticles. Langmuir 26, 8336-8346 (2010).

60 Samson, J. \& Olson, J. Some comments on the descriptions of the polarization states of waves. Geophysical Journal International 61, 115-129 (1980).

61 Greenfield, N. Analysis of the kinetics of folding of proteins and peptides using circular dichroism. Vol. 1 (2006).

62 Saptarshi, S. R., Duschl, A. \& Lopata, A. L. Interaction of nanoparticles with proteins: relation to bio-reactivity of the nanoparticle. Journal of Nanobiotechnology 11, 26-26, doi:10.1186/1477-3155-11-26 (2013).

63 Shen, J.-W., Wu, T., Wang, Q. \& Kang, Y. Induced stepwise conformational change of human serum albumin on carbon nanotube surfaces. Biomaterials 29, 3847-3855, (2008).

64 Norde, W. \& Giacomelli, C. E. BSA structural changes during homomolecular exchange between the adsorbed and the dissolved states. Journal of Biotechnology 79, 259-268 (2000).

65 Bellezza, F., Cipiciani, A. \& Quotadamo, M. A. Immobilization of myoglobin on phosphate and phosphonate grafted-zirconia nanoparticles. Langmuir 21, 11099-11104 (2005).

66 Khaleghian, A. et al. Effect of ferric oxide nanoparticles on microtubules organization. African Journal of Biochemistry Research 4, 99-104 (2010).

67 Greenfield, N. J. Circular dichroism analysis for protein-protein interactions. Protein-Protein Interactions: Methods and Applications, 55-77 (2004). 
68 Saptarshi, S. R., Duschl, A. \& Lopata, A. L. Interaction of nanoparticles with proteins: relation to bio-reactivity of the nanoparticle. Journal of nanobiotechnology 11, 26 (2013).

69 Lundqvist, M., Sethson, I. \& Jonsson, B.-H. Protein adsorption onto silica nanoparticles: conformational changes depend on the particles' curvature and the protein stability. Langmuir 20, 10639-10647 (2004).

70 Ratnikova, T. A., Nedumpully Govindan, P., Salonen, E. \& Ke, P. C. In vitro polymerization of microtubules with a fullerene derivative. ACS nano 5, 6306-6314 (2011).

71 Choudhury, D. et al. Unprecedented inhibition of tubulin polymerization directed by gold nanoparticles inducing cell cycle arrest and apoptosis. Nanoscale 5, 4476-4489 (2013).

72 Ionov, L., Bocharova, V. \& Diez, S. Biotemplated synthesis of stimuli-responsive nanopatterned polymer brushes on microtubules. Soft Matter 5, 67-71 (2009).

73 Hirokawa, N. Kinesin and dynein superfamily proteins and the mechanism of organelle transport. Science 279, 519-526 (1998).

74 Okada, Y., Yamazaki, H., Sekine-Aizawa, Y. \& Hirokawa, N. The neuron-specific kinesin superfamily protein KIF1A is a uniqye monomeric motor for anterograde axonal transport of synaptic vesicle precursors. Cell 81, 769-780 (1995).

75 Jackson, G. S. et al. Binding and hydrolysis of nucleotides in the chaperonin catalytic cycle: implications for the mechanism of assisted protein folding. Biochemistry 32, 2554-2563 (1993).

76 Schaap, I. A., Carrasco, C., de Pablo, P. J. \& Schmidt, C. F. Kinesin walks the line: single motors observed by atomic force microscopy. Biophysical journal 100, 2450-2456 (2011).

77 Kozielski, F. et al. The Crystal Structure of Dimeric Kinesin and Implications for Microtubule-Dependent Motility. Cell 91, 985-994 (1997).

78 Hoenger, A. \& Milligan, R. A. Motor domains of kinesin and ncd interact with microtubule protofilaments with the same binding geometry. J Mol Biol 265, 553-564, doi:10.1006/jmbi.1996.0757 (1997).

79 Sims, P. A. \& Xie, X. S. Probing Dynein and Kinesin Stepping with Mechanical Manipulation in a Living Cell. ChemPhysChem 10, 1511-1516, doi:10.1002/cphc.200900113 (2009). 
80 Bachand, M., Trent, A. M., Bunker, B. C. \& Bachand, G. D. Physical factors affecting kinesin-based transport of synthetic nanoparticle cargo. Journal of nanoscience and nanotechnology 5, 718-722 (2005).

81 Ramachandran, S., Ernst, K.-H., Bachand, G. D., Vogel, V. \& Hess, H. Selective Loading of Kinesin-Powered Molecular Shuttles with Protein Cargo and its Application to Biosensing. Small 2, 330-334, doi:10.1002/smll.200500265 (2006).

82 Grogan, C. et al. Characterisation of an antibody coated microcantilever as a potential immuno-based biosensor. Biosensors and Bioelectronics 17, 201-207 (2002).

83 Gennerich, A. \& Vale, R. D. Walking the walk: how kinesin and dynein coordinate their steps. Current opinion in cell biology 21, 59-67 (2009).

84 Bachand, G. D., Hess, H., Ratna, B., Satir, P. \& Vogel, V. "Smart dust" biosensors powered by biomolecular motors. Lab on a Chip 9, 1661-1666 (2009).

85 Bachand, G. D. et al. Assembly and transport of nanocrystal CdSe quantum dot nanocomposites using microtubules and kinesin motor proteins. Nano Letters 4, 817-821 (2004).

86 Doot, R. K., Hess, H. \& Vogel, V. Engineered networks of oriented microtubule filaments for directed cargo transport. Soft Matter 3, 349-356 (2007).

87 Walter, W. J., Beránek, V., Fischermeier, E. \& Diez, S. Tubulin acetylation alone does not affect kinesin-1 velocity and run length in vitro. PLoS One 7, e42218 (2012).

88 Bertalan, Z., Budrikis, Z., La Porta, C. A. \& Zapperi, S. Navigation strategies of motor proteins on decorated tracks. PloS one 10, e0136945 (2015).

89 van den Heuvel, M. G. L., de Graaff, M. P. \& Dekker, C. Molecular Sorting by Electrical Steering of Microtubules in Kinesin-Coated Channels. Science 312, 910-914, doi:10.1126/science.1124258 (2006). 


\section{Supporting Information}

\section{Elemental characterization of Sulfur-doped Carbon nanodots (S-doped C-dots)}

User-synthesized S-doped C-dots were evaluated for their physico-chemical properties. Specifically, elemental composition of the user-synthesized S-doped C-dots was evaluated using X-ray photoelectron (XPS; Physical Electronics PHI 5000 VersaProbe XPS/UPS, USA) and Energy-dispersive X-ray (EDX; JEOL JSM-7600F Scanning Electron Microscope, USA) and spectroscopies. The pass energy for XPS was maintained at $117.4 \mathrm{eV}$ with a $0.5 \mathrm{eV}$ step for survey scans, or $23.5 \mathrm{eV}$ and $0.05 \mathrm{eV}$ for detailed scans while EDX was operated at 20 $\mathrm{kV}$.

The XPS and EDX results are shown in Figure S1a and S1b; analyses revealed that the S-doped C-dots were mainly made up from carbon (C), oxygen (O), sodium (Na) and sulfur (S). A trace of phosphor was detected by XPS most likely a result of minor contamination. A detailed component analysis is shown in Table $\mathbf{S 1 .}$
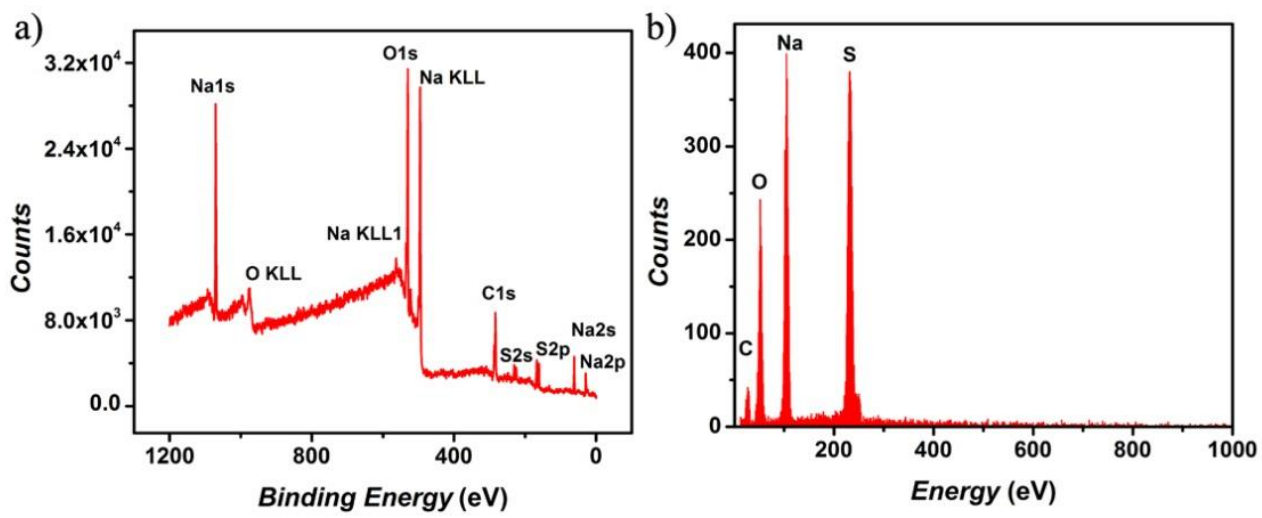

Figure S1: a) The XPS survey graph of the S-doped C-dots. b) EDX analysis of the S-doped C-dots.

\section{Table S1.}

Percentage distribution of the elements present in the user-synthesized S-doped C-dots.

\begin{tabular}{|c|c|c|c|c|c|}
\hline Element (\%) & $\begin{array}{c}\text { Carbon } \\
(\mathbf{C})\end{array}$ & $\begin{array}{c}\text { Oxygen } \\
(\mathbf{O})\end{array}$ & $\begin{array}{c}\text { Sodium } \\
(\mathbf{N a})\end{array}$ & $\begin{array}{c}\text { Sulfur } \\
(\mathbf{S})\end{array}$ & $\begin{array}{c}\text { Phosphor } \\
(\mathbf{P})\end{array}$ \\
\hline Method & 30.49 & 41.16 & 20.45 & 7.17 & 0.74 \\
\hline EDS & 23.32 & 23.08 & 28.34 & 25.26 & 0 \\
\hline
\end{tabular}




\section{Morphology and size characterization of the S-doped C-dots}

Morphologies and size of user-synthesized S-doped C-dots were assessed using transmission electron microscopy (TEM, HRTEM, Model JEM-2100) and scanning electron microscope (SEM; Hitachi High-Technologies Corporation). For TEM, the samples (1 mL of purified S-doped C-dots solution diluted into $10 \mathrm{~mL}$ of water) were dropped onto copper wire meshes and dried and dried for $20 \mathrm{~min}$ at $50{ }^{\circ} \mathrm{C}$. Subsequently, an accelerated voltage of 200 KV was used for analyses. For SEM, samples were dropped onto carbon take and analyzed at $20 \mathrm{~K}$ without any coating.

TEM and SEM results (Figure S2 a and b) identified the morphology of the S-doped C-dots and showed well-separated and quasi-spherical nanodots. S-doped C-dots also have a rather narrow size (between 2 to $10 \mathrm{~nm}$ ) with an average of about $7.18 \mathrm{~nm}$. More than $94.41 \%$ of the nanodots showed diameters of less than $12 \mathrm{~nm}$ indicating controlled synthesis.

a)

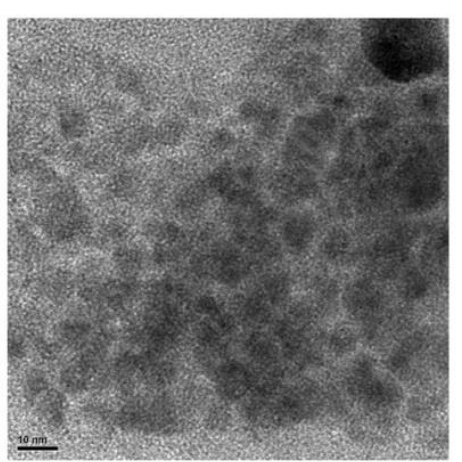

b)

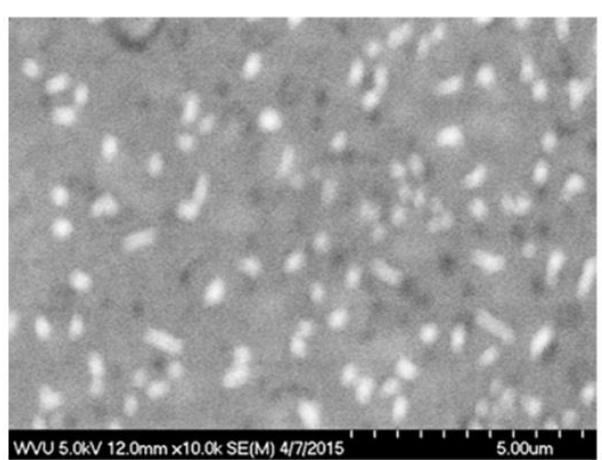

Figure S2: a) Representative TEM of S-doped C-dots. b) Representative SEM of S-doped C-dots.

\section{Spectroscopic evaluation}

Tubulin absorbance was monitored on a UV-Vis spectrophotometer (Thermo Scientific EVO300). For this, tubulin was suspended in buffered phosphate saline (100 mM, PBS, formed from a mixture of monopotassium phosphate, $\mathrm{KH}_{2} \mathrm{PO}_{4}$, and dipotassium hydrogen phosphate, $\mathrm{K}_{2} \mathrm{HPO}_{4}$, both from Fisher Scientific, USA, pH 7.4) and evaluated in the 285-345 $\mathrm{nm}$ range. Figure $\mathbf{S 3}$ shows the spectra of the protein, with no major absorbance domains in these wavelength ranges. 


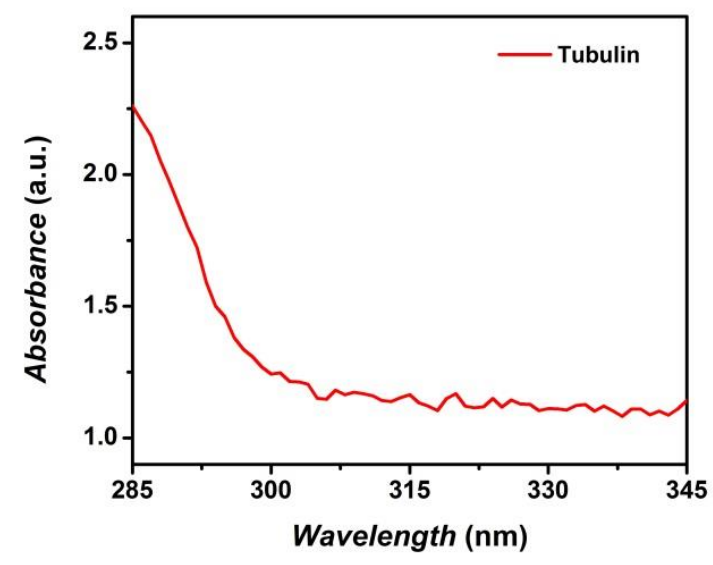

Figure S3: a) Representative absorbance spectra of the tubulin protein

\section{Atomic Force Microscopy (AFM) morphology analyses}

The cross-section morphologies of the hybrids and the microtubule were also evaluated. The height distributions were showed in Figure S4a,b. In the height distribution, the height of the baseline represented the height of the substrate, while the height of peak represented the height of the sample on the substrate. The height difference between the baseline and the peak represented the height of the sample along.

a)

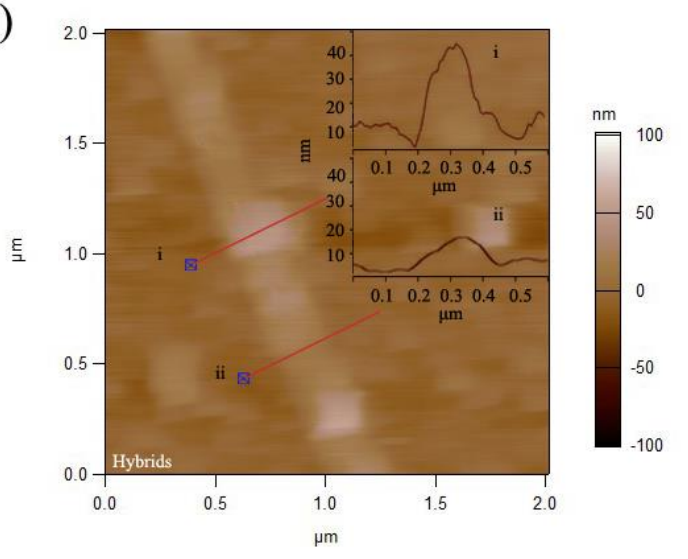

b)

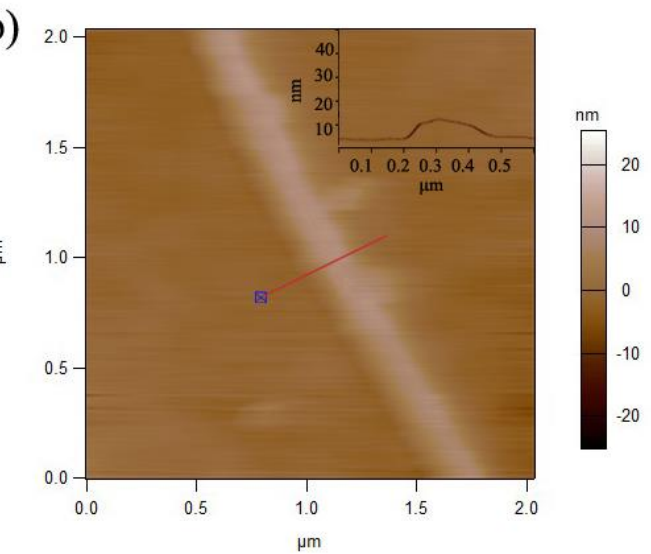

Figure S4: (a) Representative AFM analyses of kinesin-hybrid after crosslinking with glutaraldehyde. (b) Representative AFM analyses of kinesin-microtubule after crosslinking with glutaraldehyde. Inserts are showing the height distribution of samples along the red line, with the blue square representing the starting point of such analysis $(0.0 \mu \mathrm{m})$. 
The morphology and physical characteristics of the kinesin protein were evaluated using AFM with results being shown in Figure S5. For this experiment, $20 \mu \mathrm{L} 50 \mu \mathrm{g} / \mathrm{mL}$ of the sample was dropped onto the APTES functionalized mica surface and incubated for $1 \mathrm{~h}$ at room temperature. For the APTES functionalization, the mica was first cleaned with deionized water (DI water), ethanol (90\%, Fisher Scientific, USA), and again with DI water, and subsequently dried under vacuum overnight and at the room temperature. After that, the cleaned mica was subsequently functionalized with 3-aminopropyltriethoxysilane (APTES, Fisher Scientific, USA; $20 \mu \mathrm{L}, 99 \%$ ) through incubation at room temperature for $15 \mathrm{~min}$; any remaining solution was removed under vacuum. After the incubation, the surface was washed with PBS buffer thoroughly.

The AFM images were collected immediately after the washing step using contact mode in solution. For these measurements the cantilever's spring constant was calibrated using the thermal noise method; the scan rate of the tip was fixed at $0.5 \mathrm{~Hz}$ for all the experiments. At least 6 samples have been investigated to collect the sample morphology and for diameter analyses. Analysis show individual kinesin molecules deposited onto the mica surface (Figure S5a). Diameter distribution (Figure S5b) was quite large and it was presumably due to the orientation of the kinesin on the substrate.

a)

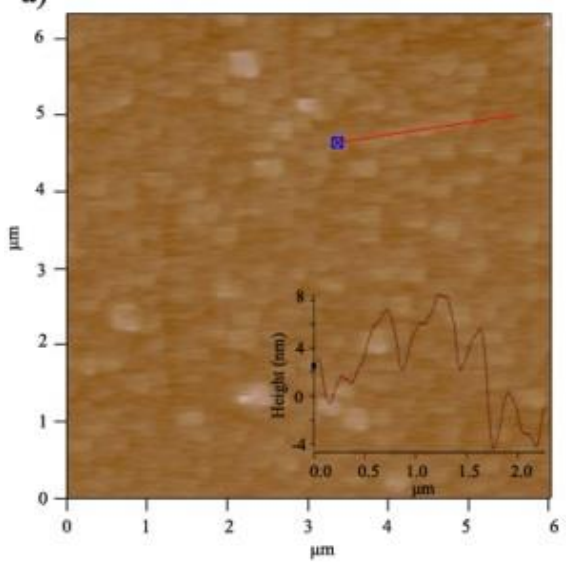

b)

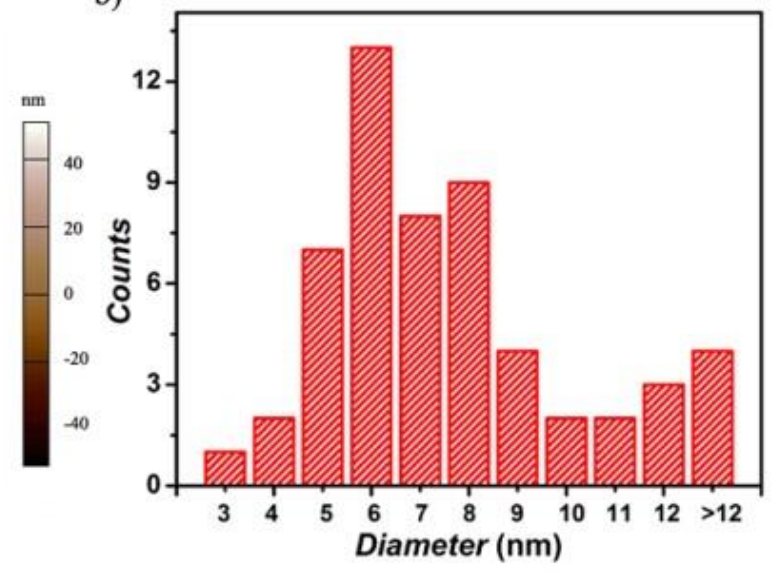

Figure S5: a) Representative AFM morphologies analyses of single kinesins molecules on the APTES-coated mica substrate. Insert shows height distribution of independent kinesins (along the red line 1) with the blue square representing the start point $(0.0 \mu \mathrm{m})$; the line followed the sample morphology. b) The diameter distribution of the kinesin as resulted from independent AFM evaluations. 


\section{Gliding motility assay}

The gliding assay was performed using established protocols. Briefly, $20 \mu \mathrm{L} 0.5 \mathrm{mg} / \mathrm{mL}$ casein (Fisher Scientific, USA) in BRB80 buffer (formed from a mixture of $80 \mathrm{mM}$ piperazine-N,N'-bis(2-ethanesulfonic acid buffer, $1 \mathrm{mM} \mathrm{MgCl}_{2}$ and $1 \mathrm{mM}$ ethylene glycol tetraacetic acid (EGTA), pH 7.3; all reagents were purchased from Fisher Scientific, USA respectively) was added into a user-made flow cell and incubated for $5 \mathrm{~min}$ at room temperature. After that, $20 \mu \mathrm{L}$ of $10 \mu \mathrm{g} / \mathrm{mL}$ kinesin solution with $0.5 \mathrm{mg} / \mathrm{mL}$ casein and $1 \mathrm{mM}$ MgATP in BRB80 buffer was also added and incubated for another $5 \mathrm{~min}$ at room temperature. Lastly, $20 \mu \mathrm{L}$ 'modified' microtubule solution (BRB80 buffer which contained $32 \mathrm{nM}$ the hybrids-Movie S1 or microtubules- Movie S2 of the equivalent concentration, $1 \mathrm{mM} \mathrm{MgATP}, 20 \mathrm{mM}$ d-glucose (Fisher Scientific, USA), $0.02 \mathrm{mg} / \mathrm{mL}$ glucose oxidase (Sigma, USA), $0.8 \mathrm{mg} / \mathrm{mL}$ catalase (Sigma, USA) and $0.5 \% \beta$-mercaptoethanol) was added and incubated for $5 \mathrm{~min}$ at room temperature. The gliding process was observed using a fluorescent microscope with a 100x objective (NA=1.4).

Movie S1: Gliding assay of the hybrids.

Movie S2: Gliding assay of the microtubules control. 
Chapter 5: Biomolecular interfaces based on self-assembly and self-recognition form biosensors capable to record single molecule binding and release

\begin{abstract}
This research proposed to create the next generation of versatile electrochemical-based biosensors capable to monitor target capture and release at a single molecule level. The biosensor integrates cellular machines (i.e., microtubules, structural elements of cells and kinesins, molecular motors involved in cellular transport) as functional units and its assembly is based on molecular self-assembly and self-recognition. Our results demonstrate that the designed biosensor was capable to allow detection of binding and unbinding events based on redox reactions at user-controlled electrode interfaces. The analysis also showed that the sensitivity of the designed biosensor or its ability to such events could be user-controlled at any given time by controlling the energy source that "fuels" the biosensors.
\end{abstract}




\section{Introduction}

Biological sensors have been widely used for disease diagnosis, environmental analysis ${ }^{1,2}$, electronic devices ${ }^{3,4}$, fermentation processes $^{5,6}$ and biodefense applications ${ }^{7,8}$. Relying on single-single molecule interactions ${ }^{9}$ or directed interactions of alternative patterns ${ }^{10}$ or $\operatorname{arrays}^{11}$ of molecules, biological sensors allow for a faster ${ }^{12,13}$ and lower cost ${ }^{14,15}$ detection while revolutionizing the sensitivity limits ${ }^{16,17}$. Further, by allowing downscaling, biological principles based on self-assembly and self-recognition could be used to biosensor's benefit to permit detection at nanometer scales ${ }^{18}$ or manipulation of nanovolumes of samples with minimum pretreatment ${ }^{19}$. Specifically, Il-HoonCho et al., ${ }^{20}$ designed an in-situ immuno-gold nanoparticle network protein-based biosensors to realize rapid detection of pathogens with a limit of detection of 3 cell $/ \mathrm{mL}$. Zhao et al., ${ }^{21}$ reported on an ultra-sensitive biosensensor for direct detection of DNA with a limit of detection of $10.9 \mathrm{aM}$; in such a set-up, no interference from unmatched, double-base mismatched or single-base mismatched targets was recorded. Nasirizadeh et al., ${ }^{22}$ showed that single mismatched and non-complementary ssDNA generate much smaller electrochemical signals than complementary ssDNA targets. Further, an acetylcholinesterase biosensor based on the prussian blue modified electrode enabled rapid detection of organophosphorous pesticides within only $10 \mathrm{~min}$ of exposure and with detection limits in $\mathrm{ng} / \mathrm{L}^{23}$. Lastly, a reduced graphene oxide modified smart conducting paper based biosensor provided a low cost, disposable system to evaluate the concentrations of carcino-embryonic antigens biomarkers ${ }^{24}$. However, even with such a realm of applications and implementation, biosensors pose implementation drawbacks either resulted from non-specific cross-binding/cross-contamination and false positives ${ }^{25}$, their inability to discriminate between viable and non-viable systems ${ }^{26,27}$, limited stability or the inhomogeneity of the sensing elements ${ }^{28}$.

Benefiting from the biological recognition and molecular assembly, cellular machines such as microtubules (MT) and kinesin I (simply called kinesin ${ }^{29}$ have been proposed as components for the next generation of biosensors capable to simplify and increase the sensitivity of detection, all under environmental conditions and controlled chemical energy. MTs are cellular cytoskeletal filaments of tubular structure and diameters of $25 \mathrm{~nm}^{30}$ with roles in cellular division and transport ${ }^{31}$ while kinesin is a MT-associated motor protein responsible of cargo trafficking ${ }^{32}$. Smart dust biosensor powered by the kinesin-MT system was used to directly detect glutathione-S-transferase $\mathrm{e}^{33}$, a phase II enzyme that catalyzes the conjugation of the reduced form of glutathione to xenobiotic substrates and is used for 
detoxification $^{34}$. Other kinesin-MT mobile immuno biosensors were used for the ultra-sensitive detection of the biowarfare Staphylococcal enterotoxin B at a $0.5 \mathrm{ng} / \mathrm{mL}$ resolution $^{35}$ or to concentrate bovine serum albumin (BSA) protein from a nearby environment at sensitivities reaching $0.5 \mathrm{nM}^{33}$. Lastly, Carroll-Portillo et al., designed a nano-harvester based on kinesin-MT system to measure tumor necrosis factor alpha $(\mathrm{TNF}-\alpha)^{36}$ a cell signaling protein used for assessing systemic inflammation ${ }^{37}$. In such proof-of-principle examples, the kinesin-MT system was not only able to concentrate the target to thus provide a lower limit of detection, but further, permitted a selective transport of such target to designated places to simplify the detection process itself. However, before a kinesin-MT biosensor becomes a viable implementation option, full control under user-specific conditions and user-friendly environments need to be demonstrated, all while downscaling sample volumes to allow for single molecule detection ${ }^{36}$.

Herein we designed the next generation of sensitive biosensors based on a kinesin-MT system capable to detect single molecule targets. Our strategy is based on creating user-functionalized, sensitive electrodes capable to evaluate and detect molecular assembly formation as refined by self-assembly and self-recognition events, all under controlled chemical energy of adenosine triphosphate (ATP) . Our analysis demonstrates not only that the proposed kinesin-MT biosensor is easy to create, fast, convenient and economic in its design and implementation, but further, that is capable to detect single binding and dissociation events. Lab-on-chip integration of such biosensor could possibly be used for signal controlling of sensitive and exquisite nanoswitches to allow storage or release of single target molecules for applications ranging from molecular diagnosis to signal transduction. 


\section{Materials and Methods}

\section{Expression of fluorescently labeled kinesin 1 molecular motor (dmKHC-EGFP) or simply kinesin}

Plasmid pPF_dmKHC-EGFP encoding for the dmKHC-EGFP protein (molecular weight $91.7 \mathrm{kDa}$ ), and consisting of the Drosophila melanogaster kinesin delta tail (dmKHC) linked to the C-terminal end of the EGFP (enhanced green fluorescent protein) his-tagged on the protein's C-terminus was obtained at West Virginia University. The specific protocol was previously published ${ }^{38}$. Briefly, the coding sequence was copied by polymerase chain reaction (PCR) from the pPK124 plasmid, a kind gift of Prof. Jonathan Howard, Yale University. A set of known primers were also used while a pTriEx-4 plasmid (Novagen, MA, USA) linearized by treatment with the NcoI endonuclease (New England Biolabs, USA) carryed out the coding sequence for a C-terminal his-tagged EGFP protein. Both the linearized pPF_EGFP plasmid and the pPK124 PCR amplicon were gel purified and assembled using a Gibson assembly kit (New England Biolabs, USA) with the assembled DNA sequences being subsequently introduced into E. Coli strain Stbl4 (Invitrogen, Fisher Scientific, USA). The pPF_dmKHC-EGFP plasmid was transformed into the E. coli strain BL21(DE3) pLysS (Stratagene, Agilent Technologies, USA) and protein expression was induced using standard protocols ${ }^{38}$.

Expressed protein was purified using two Bio-scale mini IMAC cartridges (Bio-Rad Laboratories Inc., USA) in series installed in a BioLogic DuoFlow chromatography system (Bio-Rad Laboratories Inc., CA, USA) by running two buffers, i.e., the wash buffer (50 mM sodium phosphate $\left(\mathrm{Na}_{3} \mathrm{PO}_{4}\right), 0.3 \mathrm{M} \mathrm{NaCl}, 1 \mathrm{mM} \mathrm{MgCl}_{2}, 10 \mu \mathrm{M}$ ATP, $5 \mathrm{mM}$ $\beta$-mercaptoethanol, $10 \%$ glycerol, $\mathrm{pH}$ 8.0) and the elution buffer (wash buffer with $0.5 \mathrm{M}$ imidazole, reagents from Biotool.com, USA) respectively. The protein was then eluted by imidazole gradient and concentrated into $1 \mathrm{~mL}$ volume. A second chromatographic step was applied for further purification; specifically, a Superdex 200 10/300 GL gel-filtration column (GE Healthcare Life Sciences, USA) equilibrated with storage buffer (100 mM imidazole, $300 \mathrm{mM} \mathrm{NaCl}, 1 \mathrm{mM} \mathrm{MgCl} 2,10 \mu \mathrm{M}$ ATP, $1.0 \mathrm{mM}$ 1,4-dithiothreitol (DTT), $10 \%$ sucrose, pH 7.0) was used. Lastly, the protein concentration was estimated using the Coomassie protein assay and bovine gamma globulin standard (reagents from Fisher Scientific, USA). 


\section{Electrode cleaning}

A gold electrode ( $\mathrm{CH}$ Instrument Inc., USA, diameter $2 \mathrm{~mm}$ ) was treated with Piranha solution (containing $96.4 \%$ sulfuric acid, $\mathrm{H}_{2} \mathrm{SO}_{4}$, and $30 \%$ hydrogen peroxide, $\mathrm{H}_{2} \mathrm{O}_{2}$, Fisher Scientific, USA in a $3: 1(\mathrm{v}: \mathrm{v}))$ for $10 \mathrm{~min}$ to remove any organic contaminant ${ }^{39}$. The electrode was subsequently rinsed with deionized water (DI water) and then successively polished with $1.0 \mu \mathrm{m}, 0.3 \mu \mathrm{m}$ and $0.05 \mu \mathrm{m} \alpha$-alumina $\left(\alpha-\mathrm{Al}_{2} \mathrm{O}_{3}, \mathrm{CH}\right.$ Instrument Inc., USA) powders. To remove impurities resulted from polishing, the electrode was also rinsed with DI water, $10 \mathrm{~mL}$ ethanol (90\%, Fisher Scientific, USA), acetone (99.7\%, Fisher Scientific, USA) and again DI water, all under water bath sonication conditions. The electrode was subjected to cleaning by cyclic voltammetry in $50 \mathrm{mM} \mathrm{H}_{2} \mathrm{SO}_{4}$ with cycles being ran until a stable scan was obtained ${ }^{39}$. Lastly, the cleaned electrode was again rinsed thoroughly with DI water and used for the experiments listed below.

\section{Electrochemically functionalized kinesin}

Ferrocene monocarboxylic acid-kinesin conjugates were prepared by dissolving $4 \mathrm{mg}$ ferrocene monocarboxylic acid (FCA, Fisher Scientific, USA) in $800 \mu \mathrm{L}$ of BRB80 buffer (formed from a mixture of $80 \mathrm{mM}$ piperazine-N,N'-bis(2-ethanesulfonic acid buffer, $1 \mathrm{mM}$ $\mathrm{MgCl}_{2}$ and $1 \mathrm{mM}$ ethylene glycol tetraacetic acid (EGTA), $\mathrm{pH}$ 6.8; all reagents were purchased from Fisher Scientific, USA) which contained $5 \mathrm{mM}$ biotin-sulfo-N-Hydroxy succinimide (biotin-sulfo-NHS, Sigma, USA ), 2 mM 1-Ethyl-3-(3'-dimethylaminopropyl) carbodiimide (EDC, Fisher Scientific, USA) and $10 \mu \mathrm{M}$ taxol (Fisher Scientific, USA). The mixture was subsequently incubated for $15 \mathrm{~min}$ at room temperature. Upon incubation, $90 \mu \mathrm{L}$ of $2.8 \mathrm{mg} / \mathrm{mL}$ kinesin (expressed as previously described) was mixed with the FCA solution and incubated for $4 \mathrm{~h}$ at $4{ }^{\circ} \mathrm{C}$; the reaction was terminated by adding $\beta$-mercaptoethanol (Fisher Scientific, USA, $20 \mathrm{mM}$ final concentration) ${ }^{40}$.

FCA loading was estimated by subtracting the amount of the free FCA in the supernatant of the span down FCA-kinesin conjugates from the initial amount of FCA offered in the binding reaction. Specifically, the FCA-kinesin conjugates were span at 30,000 rpm for 10 min on an Allegra 64R centrifuge (Beckman Coulter, USA) and the supernatant and washes of the different span down steps were recorded and evaluated using using a fluorescent microscope (Nikon, USA) and a 100 x objective $(\mathrm{NA}=1.4)$ under a GFP filter and under an exposure time of $8.3 \mathrm{~s}$. The concentration of the free FCA in the supernatant was also 
measured using an electrochemical workstation ${ }^{41}$ (VersaSTAT 3 potentiostat/galvonostat, Princeton Applied Research, USA) by recording the peak currents at a scan rate of $50 \mathrm{mV} / \mathrm{s}$.

\section{Microtubule synthesis}

Microtubules (MTs) were synthesized from free tubulin suspended in a polymerization solution according to established protocols ${ }^{42,43}$. Briefly, the polymerization solution was obtained by vortexing $5 \mu \mathrm{L} 100 \mathrm{mM} \mathrm{MgCl}_{2}$, with $6 \mu \mathrm{L}$ dimethyl sulfoxide (DMSO, 99.7\%, Fisher Scientific, USA), $5 \mu \mathrm{L} 25 \mathrm{mM}$ guanosine-5'-triphosphate (GTP, Sigma, USA) and 9 $\mu \mathrm{L}$ BRB80 buffer. Then $2.5 \mu \mathrm{L}$ of this polymerization solution was injected into $10 \mu \mathrm{L}$ of 4 $\mathrm{mg} / \mathrm{mL}$ tubulin (Cytoskeleton Inc, USA) with the subsequent mixture being incubated at 37 ${ }^{\circ} \mathrm{C}$ for $30 \mathrm{~min}$. The resulting MTs were stabilized in $1 \mathrm{~mL} \mathrm{BRB} 80$ buffer solution containing $10 \mu \mathrm{M}$ taxol. The stabilized MTs were kept at room temperature until their experimental use; fresh solutions were prepared if the initial MT solution was older than 3 days.

\section{FCA-kinesin binding to MTs in a user-controlled synthetic environment}

FCA-kinesin conjugates were incubated with MTs in the presence of adenylyl-imidodiphosphate (AMP-PNP, Sigma, USA) to form FCA-kinesin-MT assemblies. Specifically, FCA-kinesin conjugates (different concentrations in BRB80 buffer were tested, i.e., 13.2-330 nM) were mixed with $2 \mu \mathrm{L}$ of $20 \mathrm{mM}$ AMP-PNP and incubated for $1 \mathrm{~h}$ at $4{ }^{\circ} \mathrm{C}$. Subsequently, the mixture was mixed with $20 \mu \mathrm{L}$ MT solution and incubated for another 30 min, again at room temperature. Any free FCA-kinesin was removed by spinning the assemblies at 30,000 rpm for $10 \mathrm{~min}$ and washing of the resulting pellet with BRB80 buffer. The supernatant was evaluated using fluorescence microscopy (100 x objective $(\mathrm{NA}=1.4)$, GFP filter, exposure time of $8.3 \mathrm{~s}$ ) to trace the amount of unbound FCA-kinesin; the procedure was repeated until no free FCA-kinesin was observed. The pellet (FCA-kinesin-MT assemblies) was redissolved in $20 \mu \mathrm{L} \mathrm{BRB80} \mathrm{buffer} \mathrm{with} 10 \mathrm{mM}$ taxol and used immediately.

\section{Functionalization of the electrode}

The 11-mercaptoundecanoic acid (Fisher Scientific, USA) was used to form a self-assembled monolayer (SAM) on the electrode previously cleaned with Piranha solution (see above). SAM was prepared by immersing the cleaned electrode overnight into a $100 \mathrm{mM}$ 11-mercaptoundecanoic acid solution in ethanol. Subsequently, $50 \mu \mathrm{g} / \mathrm{mL}$ anti-tubulin 
antibody (Sigma, USA) was attached to the electrode using the 1-Ethyl-3-(3-dimethylaminopropyl)carbodiimide (EDC)/ N-Hydroxysuccinimide (NHS) zero chemistry $^{44}$. For this, $50 \mu \mathrm{L}$ EDC/NHS $(0.4 \mathrm{mM} / 0.1 \mathrm{mM})$ solution was dropped onto the gold electrode and incubated at room temperature for $15 \mathrm{~min}$. The electrode was subsequently washed with $1 \mathrm{~mL}$ BRB80 buffer; $50 \mu \mathrm{L}$ solution of $20 \mu \mathrm{g} / \mathrm{mL}$ anti-tubulin antibody was then dropped onto the electrode and the electrode was again incubated at room temperature for $4 \mathrm{~h}$. After this additional incubation step, the electrode was washed with BRB80 and subsequently exposed to $50 \mu \mathrm{L}$ FCA-kinesin-MT assemblies for $1 \mathrm{~h}$ incubation, all at room temperature. Lastly, the electrode was rinsed with BRB80 buffer containing $10 \mathrm{mM}$ taxol and then exposed to $50 \mu \mathrm{L}$ of $100 \mathrm{mM}$ bovine serum albumin (BSA, Fisher Scientific, USA) for 30 min, at the room temperature. After this last incubation step, the electrode was rinsed with BRB80 containing $10 \mathrm{mM}$ taxol and subsequently used for cyclic voltammetry experiments (see below).

\section{Electrochemical measurement}

A three-electrode system was used to run the cyclic voltammetry experiments. The working electrode was the bare or functionalized gold electrode (FCA-kinesin-MT/11-mercaptoundecanoid acid/Au), the silver/silver chloride (Ag/AgCl, 1.0 $\mathrm{M} \mathrm{KCl}$ ) was the reference electrode, while the platinum electrode served as the counter-electrode (all electrodes were purchased from $\mathrm{CH}$ Instrument, USA). The reference electrode and the counter-electrode were rinsed with DI water and blown dry before every use. Cyclic voltammetry (CV, VersaSTAT 3 potentiostat/galvonostat, Princeton Applied Research, USA) was carried out in BRB80 buffer ( $\mathrm{pH}$ 6.8) containing $10 \mathrm{mM}$ taxol. The scan rate varied from $6 \mathrm{mV} / \mathrm{s}$ to $300 \mathrm{mV} / \mathrm{s}(6 \mathrm{mV} / \mathrm{s}, 8 \mathrm{mV} / \mathrm{s}, 10 \mathrm{mV} / \mathrm{s}, 20 \mathrm{mV} / \mathrm{s}, 30 \mathrm{mV} / \mathrm{s}, 40 \mathrm{mV} / \mathrm{s}$, $50 \mathrm{mV} / \mathrm{s}, 60 \mathrm{mV} / \mathrm{s}, 70 \mathrm{mV} / \mathrm{s}, 80 \mathrm{mV} / \mathrm{s}, 90 \mathrm{mV} / \mathrm{s}, 100 \mathrm{mV} / \mathrm{s}, 200 \mathrm{mV} / \mathrm{s} 300 \mathrm{mV} / \mathrm{s}$.). Electrochemical impedance spectroscopy (EIS) was carried out on the same instrument, in BRB80 buffer containing $50 \mathrm{mM}$ potassium ferricyanide $\left(\mathrm{K}_{3} \mathrm{Fe}(\mathrm{CN})_{6}\right.$, Fisher Scientific, USA) and $10 \mathrm{mM}$ taxol and in a frequency range from $0.1 \mathrm{~Hz}$ to $100 \mathrm{kHz}$.

\section{Statistics}

For each concentration of FCA-kinesin conjugates, 6 modified electrodes were used to obtain 6 different $\mathrm{CV}$ graphs which were subsequently statistically assessed. The total length of the MTs on the electrode was estimated using the area of the electrode $\left(3.14 \mathrm{~mm}^{2}\right)$ divided 
by the diameter of an individual MT $(25 \mathrm{~nm})$ and assuming that all of the electrode's surface was covered by a MT monolayer. The FCA covalent binding to the amino groups of the kinesin allowed determination of the theoretical loading, with the kinesin structure being imported from previous research ${ }^{45}$ (included in Supporting Information S1). 


\section{Results and Discussion}

The recognition process between kinesin and microtubule (MT) as well as kinesin's movement along a MT track have been mimicked in synthetic environment for transport-oriented tasks, all under the chemical energy of adenosine triphosphate (ATP) ${ }^{42,43}$. Viable and remote ${ }^{46}$ kinesin-MT biosensors capable of specific capturing ${ }^{47}$ or transport of antigens ${ }^{48}$ have been proposed for biomedical and biodefense research ${ }^{49}$ or for smart dust biosensors generation ${ }^{50}$. However, in such applications the reality of implementation of such a kinesin-MT system was hindered by the biosensor's reduced specificity and overall analyte-related selectivity ${ }^{51}$. Herein we proposed to design the next generation of kinesin-MT biosensors that allow autonomous and controlled detection of molecular analytes binding and unbinding events, all with single molecule resolution, on cheap and easy to manufacture electrochemical platforms. It is envisioned that by controlling the recognition of kinesin-MT binding events with high sensitivity and accuracy one could allow the interpretation and integration of the system's transport function into a scalable chip-based platform that permits detection at low power of consumption and under low fabrication $\operatorname{cost}^{52,53}$.

To demonstrate the feasibility of the proposed design, we first labeled lab-expressed kinesin with a pseudo-reference agent capable of producing an electrochemical signal, namely ferrocene monocarboxylic acid (FCA; Figure 1a) ${ }^{3}$. Published reports showed that FCA could be bound to proteins either through electrostatic interactions or direct insertions ${ }^{54}$ at their terminal amine sites or reactive amino acid side chains ${ }^{55}$. Further, previous reports showed that FCA has two known pair of reversible redox peaks between $0.2 \mathrm{~V} \sim 0.8 \mathrm{~V}^{56}$.

Kinesin's successful FCA labeling was demonstrated using cyclic voltammetry (CV); specifically, a pair of peaks between $0.2 \mathrm{~V} \sim 0.8 \mathrm{~V}$ were recorded for the FCA-kinesin conjugates, all relative to unlabeled kinesin (Figure 1b; concentration of kinesin in both FCA-kinesin conjugates as well as in unlabeled kinesin was $1.5 \mu \mathrm{M})$. The observed pair of redox peaks was presumably due to the redox reaction of the FCA in the conjugates which had the reduction peak at about $0.25 \mathrm{~V}$ and the oxidation peak at about $0.32 \mathrm{~V}$. A reduction peak current was also observed at $4.93 \mu \mathrm{A}$ with the corresponding oxidation peak current at $7.75 \mu \mathrm{A}$. 


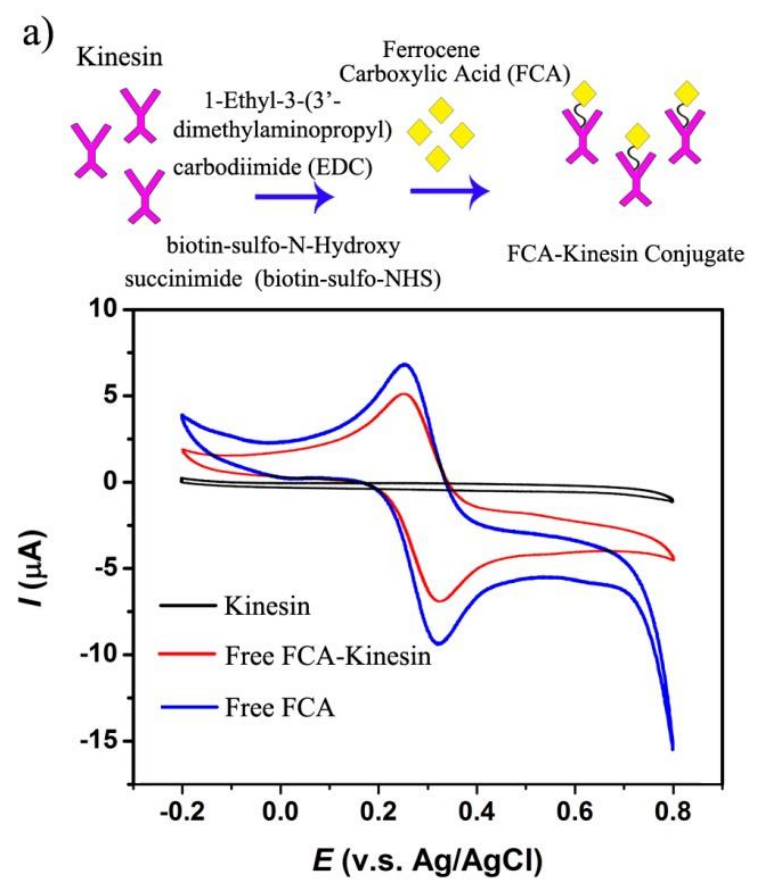

Figure 1: a) Schematic illustration of kinesin functionalization with ferrocene monocarboxylic acid (FCA). b) The cyclic voltammetry (CV) of unlabeled kinesin (simply called kinesin), FCA-kinesin conjugates and free FCA.

Theoretical estimate of the FCA molecules bound at the available amino groups on the kinesin was 192 FCA per one kinesin molecule ${ }^{45}$. However, the experimental value, i.e., FCA loading estimated by subtracting the amount of the free FCA in the supernatant obtained by washing the FCA-kinesin conjugates (Supporting Information S2) and evaluated using CV was about 1245 FCA molecules per individual kinesin molecule. The difference is presumably due to the non-specific physical binding of the $\mathrm{FCA}^{57}$ to the kinesin molecules.

FCA-kinesin conjugates were subsequently interfaced with user-synthesized MTs in the presence of adenylyl-imidodiphosphate (AMP-PNP; Figure 2a) to form FCA-kinesin-MT assemblies. MTs were obtained from precursor tubulin polymerized under the chemical energy of guanosine triphosphate (GTP) ${ }^{42}$. AMP-PNP is a non-hydrolyzed form of adenosine triphosphate (ATP) that allows for the high-affinity binding reactions between kinesin and MT to occur ${ }^{58}$. Previous studies showed that both heads of an individual kinesin bind at about $8 \mathrm{~nm}$ apart onto a single MT profilament either in the presence of AMP-PNP or its ATP counterpart $^{59,60}$. Free FCA-kinesin was removed by centrifugation as previously described. The FCA-kinesin-MT assembly was subsequently immobilized onto anti-tubulin antibodies covalently immobilized onto the gold electrode functionalized with a self-assembled monolayer (SAM) (Figure 2b) ${ }^{61}$. Herein SAM was used to create a "soft" spacer ${ }^{62}$ between 
the bare gold electrode surface and the FCA-kinesin-MT assembly, with such spacer also expected to ensure a more controlled and localized immobilization ${ }^{63}$ while reducing non-specific binding and denaturation of the individual proteins forming the assembly ${ }^{64}$.

A range of different concentrations of FCA-kinesin were initially tested to identify any changes in the redox potential and how they were related to the number of FCA-based conjugates immobilized at the MT interface (Figure 2c). A direct dependence of the peak currents on the FCA-kinesin conjugates concentrations was observed; specifically, the reduction peak currents decreased from $1.78 \mu \mathrm{A}$ to $0.49 \mu \mathrm{A}$ while the oxidation peak currents decreased from $2.14 \mu \mathrm{A}$ to $0.58 \mu \mathrm{A}$ when the FCA-kinesin conjugates concentrations used were varied from 13.2 to $84.3 \mathrm{nM}$.

Electrode surface functionalization was confirmed using electrochemical impedance spectrum (EIS) with analyses being shown in Figure 2d. Specifically, the Nyquist plot that plots the relationship between the real parts of the impedance $\left(Z_{R}\right)$ and the imaginary parts of the impedance $\left(\mathrm{Z}_{\mathrm{I}}\right)^{65}$ of the bare gold electrode (Au; black) was a straight line, representative of the clean and flat surface of the electrode ${ }^{66}$. Upon SAM functionalization however, the Nyquist plot changed to display a semicircle-like shape (charge-transfer resistance $R_{c t}<10 \Omega$, where $R_{c t}$ the current flow produced by redox reactants at the interface $^{65}$ reflective of the increase in impedance as resulted from the 11-mercaptoundecanoid Acid (MUA)/Au coverage ${ }^{67}$. Additional functionalization with anti-tubulin antibody changed the shape even further, with the $\mathrm{R}_{\mathrm{ct}}$ value reaching about $80 \Omega$ for the anti-tubulin antibody/MUA/Au ${ }^{68}$. Lastly, the $\mathrm{R}_{\mathrm{ct}}$ increased to about $100 \Omega$ after the bovine serum albumin (BSA) functionalization. The impedance decreased to about $50 \Omega$ after immobilization of the FCA-kinesin-MT assembly onto the electrode surface (to form FCA-kinesin-MT/Anti-tubulin Antibody (BSA)/MUA/Au) presumably due to the redox reactions associated with FCA. Herein the chosen concentration of FCA-kinesin-MT was 84.3 nM (see Figure 2c). 
a)

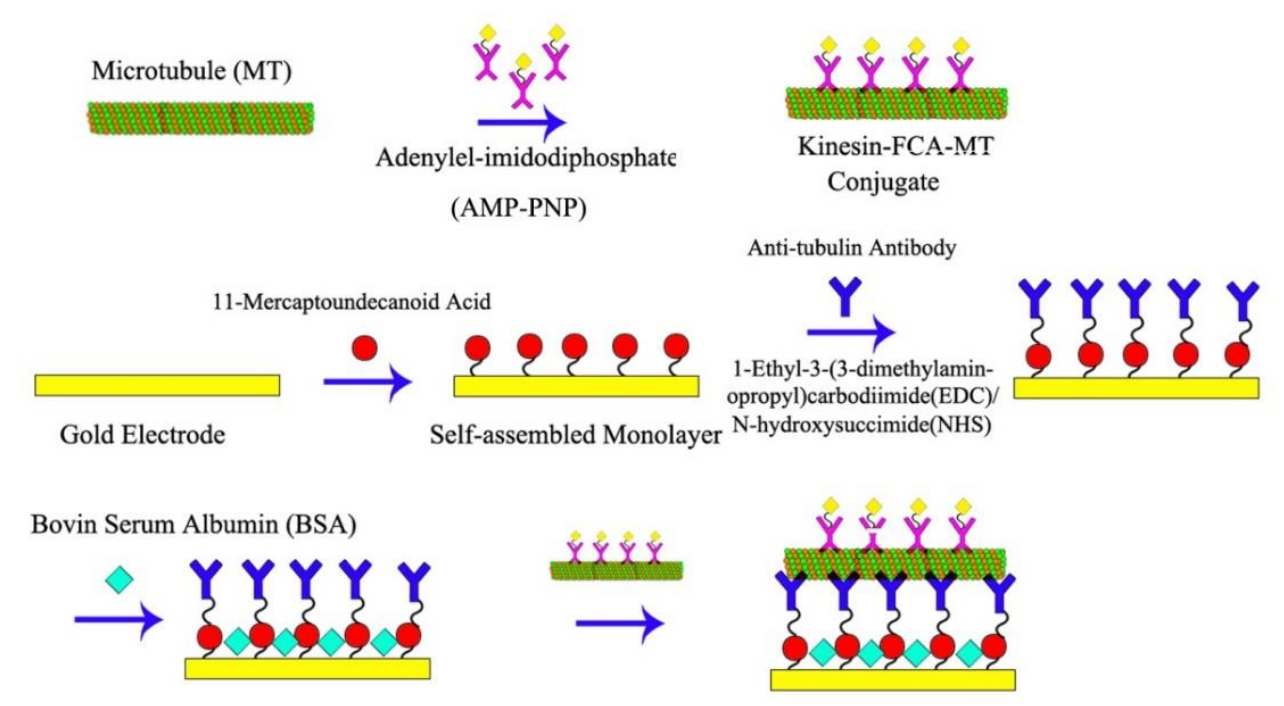

c)

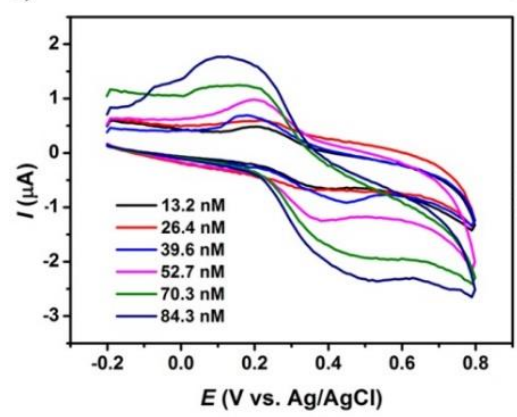

d)

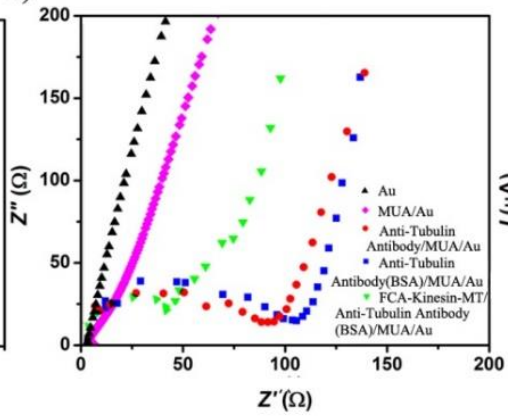

e)

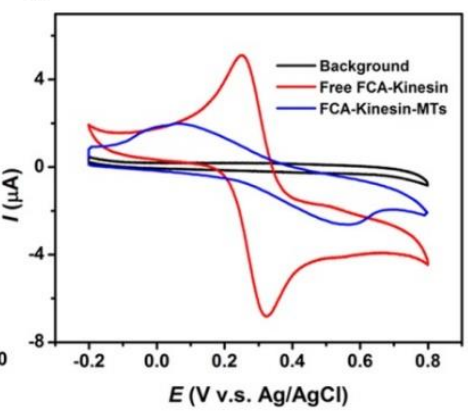

Figure 2: a) Schematic illustration of FCA-kinesin conjugate binding to a microtubule (MT) to form a FCA-kinesin-MT assembly in the presence of adenylyl-imidodiphosphate (AMP-PNP). b) The assembly was immobilized onto an anti-tubulin antibody functionalized electrode. Prior immobilization, surface functionalization of the electrode with self-assembly monolayers of 11-mercaptoundecanoic acid (MUA) and bovine serum albumin (BSA) aimed to reduce non-specific binding, was used. c) The CV analysis of different concentrations of FCA-kinesin-MT assembly immobilized at the modified electrode interface. d) Electrochemical impedance spectrum (EIS) at the functionalized electrode interface. Black: bare gold electrode (Au). Pink: MUA/Au. Red: Anti-tubulin Antibody/MUA/Au. Blue: Anti-tubulin Antibody (BSA)/MUA/Au. Green: FCA-kinesin-MT/Anti-tubulin Antibody (BSA)/MUA/Au. e) $\mathrm{CV}$ of background (black; obtained at the modified electrode (Anti-tubulin Antibody (BSA)/MUA/Au) interface), FCA-kinesin-MT (red) at the modified electrode interface, free FCA (blue) at the anti-tubulin antibody functionalized electrode interface and free FCA-kinesin control (pink) at the anti-tubulin antibody functionalized electrode (Anti-tubulin Antibody (BSA)/MUA/Au) interface respectively. 
The detection ability is shown in Figure 2e; for this, the voltammetric waves of the modified electrode interface (black curve), the FCA-kinesin-MT assembly (red curve), free FCA-kinesin conjugate (pink curve) and free FCA (blue curve) were studied at the modified electrode interface, i.e., Anti-tubulin Antibody (BSA)/MUA/Au under a fixed scan rate of 50 $\mathrm{mV} / \mathrm{s}$. The chosen scan rate was based on previous studies which showed that such value provide a smooth profile of most redox reactions ${ }^{69}$. As showed, well-defined pairs of redox peaks were identified for each sample except the background; reduction potentials of about $0.255 \mathrm{~V}$ and oxidation potentials of about $0.322 \mathrm{~V}$ were identified for the free FCA and FCA-kinesin conjugate respectively. Although FCA-kinesin had similar redox potential as free FCA, the reduction current decreased from 6.64 to $4.89 \mu \mathrm{A}$ while the oxidation current decreased from 10.1 to $7.83 \mu \mathrm{A}$. The reduction potential of the FCA-kinesin-MT assembly was about $0.08 \mathrm{~V}$ while its oxidation potential was about $0.51 \mathrm{~V}$.

Our analysis confirm previous reports that showed that ferrocene $(\mathrm{Fc})$ derivatives have redox peaks at about $0.15 \mathrm{~V}$ and $0.26 \mathrm{~V}$ (for $\mathrm{Fc}-\mathrm{CH}_{2} \mathrm{OH}$ ) or $0.25 \mathrm{~V}$ and $0.6 \mathrm{~V}$ for $\mathrm{Fc}_{\mathrm{C}} \mathrm{CHO}^{70}$ respectively. The observed oxidation peak current of the FCA-kinesin-MT assembly was about $1.86 \mu \mathrm{A}$ while its reduction peak current was about $2.56 \mu \mathrm{A}$. These results are similar to previous reports that showed that the FCA has redox peaks between $0.2 \sim 0.4 \mathrm{~V}$ at a bis(4-pyridyl) disulphide (PySSPy)/glucose oxidase-modified gold electrode ${ }^{71}$. The control black curve appeared smooth with no obvious peaks in the voltage range of FCA thus confirming that there was no redox reaction happening at such interface ${ }^{72}$.

Our analysis also showed that the separation of the redox peaks $(\Delta \mathrm{Ep} ; 0.468 \mathrm{~V})$ for the FCA-kinesin-MT assembly was much larger than the one of the free FCA or the FCA-kinesin conjugates respectively, all as recorded at the modified gold electrode $(0.059 \mathrm{~V})$. The recorded peak separation possibly indicates a decrease of the reversibility of the redox reaction and/or a faster electron transfer kinetics at the electrode interface. For the first, the non-specific binding the FCA onto the kinesin could possibly lead to embedment of its electrochemical activity and active site coverage as resulted from the overall kinesin hindrance effects. This is supported by previous analysis by $\mathrm{Wu}$ et al., ${ }^{73}$ that showed that the electrochemical signal of the alizarin red S (ARS) decreased rapidly after it was specifically bound to BSA, with such bound molecule getting buried/ entrapped into the protein; ARS is an organic dye used as an electrochemical probe ${ }^{74}$. The electrochemical signal of the ARS was however fully recovered when the molecule was exposed upon unfolding BSA by addition of urea. For the second, the increased peak separation could be attributed to the 
electrode modification ${ }^{75}$. In particular, Chen et al., ${ }^{76}$ found that chemical derivation of the carbonyl groups on the electrode would decrease the electron transfer rate of the $\mathrm{Fe}^{3+} /^{2+}$ pair by 2 3 orders of magnitude. Further, when compared with the free FCA, the redox peaks of the conjugates and also the assembly appeared to be broader with the broadening possibly indicating that the electrochemical process became less reversible, and the electrochemical reversibility factor $f_{r}$ approaches a zero value ${ }^{77}$. Such behavior is presumably due to the electrochemical activity sites in the FCA being concealed after FCA binding to kinesin ${ }^{73}$.

Upon demonstrating that both conjugates and assemblies as well as their association in the AMP-PNP state could be detected at functionalized gold electrodes, we evaluated the sensitivity of such detection. Herein sensitivity is defined as the ability of the functionalized electrode to discriminate between different concentrations of FCA-based conjugates immobilized onto similar concentrations of MTs. The relationship between the anodic peak currents and the concentrations of the FCA-kinesin conjugates immobilized onto the MTfunctionalized electrode is shown in Figure 3a. To record such sensitivity, we extended the range of concentrations of FCA-kinesin conjugates from 13.2 to $330 \mathrm{nM}$. It was observed that in this concentrations range the anodic peak currents increased from 0.45 to $1.79 \mu \mathrm{A}$ presumably due to the increase in the number of FCA-kinesin conjugates present at specific MT binding sites. This was further confirmed by the lower concentration ranges of the cathodic peak currents which also increased rapidly, i.e., from 0.579 to $1.92 \mu \mathrm{A}$. Our analysis also showed that in the higher concentration range (from $84.3 \mathrm{nM}$ to $330 \mathrm{nM}$ ), the anodic peak currents did not seem to change relative to the increase in the FCA-kinesin conjugates concentration. This was presumably due to all the available binding sites on the MT being fully covered by the conjugates. Indeed, theoretical calculation of the maximum number of FCA-kinesin to be bound onto a monolayer of MTs at the electrode interface led to about $1.58 \times 10^{12}$ FCA-kinesin conjugates. Herein the monolayer was considered to be formed from the total length of a MT covering the overall electrode area divided by an individual MT diameter (i.e., $25 \mathrm{~nm})^{78}$ as well as considering that kinesin molecules binding area is about 8 $\mathrm{nm}$ at a MT individual binding $\operatorname{site}^{79}$ (Supporting information S3). The number of FCA-kinesin conjugates in $13.2 \mathrm{nM}$ concentration was about $5.66 \times 10^{11}$, in $84.3 \mathrm{nM}$ was about $3.62 \times 10^{12}$, while at the $330 \mathrm{nM}$ was about $1.45 \times 10^{13}$ respectively. For such calculations we considered the molecular weight of kinesin as being $188 \mathrm{kDa}^{29}$ and the Avogadro number $6.0221409 \times 10^{23}$. In addition, as showed in Figure 3a inset, there was a linear relationship between the reduction peak currents and the FCA-kinesin concentrations 
in the concentration range of $13.2 \mathrm{nM}$ to $39.5 \mathrm{nM}$. Based on these above and considering that the signal to noise ratio needs to equals 3 in such $\operatorname{cases}^{80}$, the calculated limit of detection of FCA-kinesin was $6.26 \mathrm{nM}$, while the sensitivity was $0.38 \mathrm{nM}$ according to the linear fitting.

Figure 3b shows the dependence of the charge (Q) at the electrode surface on the different concentrations of the FCA-kinesin being used. Herein the charge was calculated based on Faraday's law ${ }^{81}$ using:

$$
Q=I \times t
$$

where $\mathrm{I}$ is the current and $\mathrm{t}$ is the time necessary for a redox reaction to occur. Analyses revealed that $\mathrm{Q}$ increased from $0.71 \mu \mathrm{C}$ to $1.87 \mu \mathrm{C}$ with increasing the immobilized FCA-kinesin conjugates concentrations at the MT interfaces, with such an increase being visually divided into two separate regions. Specifically, within $13.2 \mathrm{nM}$ to $84.3 \mathrm{nM}$ FCA-kinesin concentrations, Q increased rapidly. However, upon reaching the saturated concentration of $84.3 \mathrm{nM}$ for the FCA-kinesin conjugates, Q values seemed to have reached a plateau and subsequently only change slightly presumably due to the saturation levels $\left(\mathrm{Q}_{\text {sat }}\right)$ associated with full coverage of the MT monolayer ${ }^{57}$ or non-specific binding at the electrode itself to hinder the FCA signal. In particular, previous research has showed that the possible generation of reactive oxygen species via Haber-Weiss and/or Fenton reactions ${ }^{82}$ could lead to possible hindrance ${ }^{73}$ of the FCA signal or kinesin denaturation ${ }^{83}$ respectively. 
a)

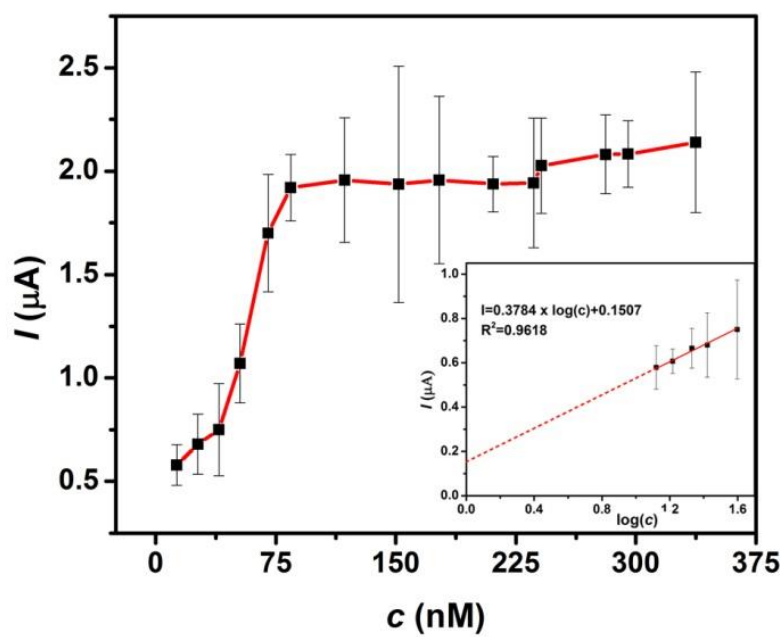

b)

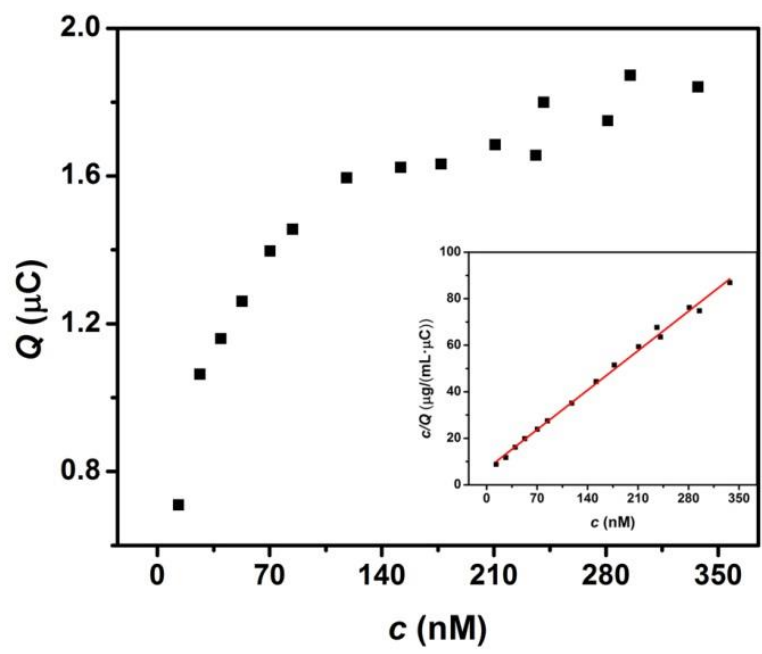

Figure 3: a) The relationship between the anodic peak currents and the concentrations of the FCA-kinesin-MT assemblies immobilized onto the functionalized electrode. Inset: The linear relationship between the peak currents and the concentrations of the FCA-kinesin-MT assemblies immobilized onto the electrode. b) The relationship of the charge involved in the redox reaction $(\mathrm{Q})$ at the modified electrode and the concentrations of the FCA-kinesin-MT assemblies. Inset: The linear relationship between the $\mathrm{c} / \mathrm{Q}$ and the concentrations of FCA-kinesin. All experiments were performed at a scan rate of $50 \mathrm{mV} / \mathrm{s}$.

Based on the obtained charge listed in Figure 3b, the number of available reactants (i.e., FCA) that could undertake a redox reaction at the electrode interface can also be calculated from the Faraday's law ${ }^{84}$ by using:

$Q=n F A \Gamma_{F C A}$ 
where $\mathrm{Q}$ is the charge involved in the reaction or the accumulated charge on the electrode surface, $\mathrm{n}$ is the number of the electron transferred per molecule that undergoes the redox reaction ( 1 in this study) ${ }^{85}, \mathrm{~F}$ is the Faraday constant $\left(9.64870 \times 10^{4} \mathrm{C}^{\circ} \mathrm{mol}^{-1}\right)$, A is the area of the interface and $\Gamma$ is the surface coverage of the reactant (i.e., FCA in this study). Specifically, the surface coverage of the kinesin $\left(\Gamma_{\text {kinesin }}\right)$ was calculated as:

$\Gamma_{\text {kinesin }}=\frac{N_{\text {kinesin }}}{N_{F C A}} \Gamma_{F C A}$

where $\mathrm{N}_{\text {kinesin }} / \mathrm{N}_{\mathrm{FCA}}$ represented the binding ratio between kinesin and FCA in the FCA-kinesin conjugates respectively. Lastly, considering that surface coverage of the FCA on the electrode was $3.90 \times 10^{16}$ molecules $/ \mathrm{m}^{2}\left(1.22 \times 10^{13}\right.$ molecules on the electrode in total if all the electrode area was covered by a MT monolayer) at the saturated concentration (Figure 3b), and considering that modification of kinesin with FCA would not considerably increase its surface area, the surface coverage of the FCA-kinesin was about $3.13 \times 10^{13}$ molecules $/ \mathrm{m}^{2}$ $\left(9.82 \times 10^{9}\right.$ molecules on the electrode in total) at the saturated concentration compared with $5.09 \times 10^{15}$ molecules $/ \mathrm{m}^{2}\left(1.57 \times 10^{12}\right.$ molecules $)$ of the theoretical full coverage value of the electrode.

We further evaluated whether the binding affinity (i.e., recognition reaction between kinesin and microtubule) changes upon kinesin labeling with FCA. For this, we used the Langmuir model ${ }^{86}$,

$\frac{c}{Q}=\frac{c}{Q_{s a t}}+\frac{1}{K Q_{s a t}}$

where $\mathrm{c}$ was the concentration of the redox cation (the concentration of the FCA-kinesin conjugate in this study), $\mathrm{K}$ is the binding constant and $\mathrm{Q}_{\text {sat }}$ is the charge involved in the redox reaction at the saturated FCA-kinesin conjugates coverage respectively. Previous research has showed that this model can be used to study the binding process between $\left(\mathrm{Ru}\left(\mathrm{NH}_{3}\right)_{6}{ }^{3+}\right)$ and DNA molecules on the electrode surface ${ }^{38}$ for instance. Our analysis showed that there was a linear relationship between the c/Q and the concentration of the immobilized FCA-kinesin conjugates that allowed calculation of the $\mathrm{Q}_{\text {sat }}$ and $\mathrm{K}$ respectively. Specifically, Figure $\mathbf{3 b}$ inset showed the linear relationship between the c/Q and the kinesin concentration and allow 
calculation of the saturated charge as being $1.96 \mu \mathrm{Q}$.

Electron transfer kinetics or the influence of the scan rate on the $\mathrm{CV}$ waves was also investigated. Previous research has showed that electron transfer kinetics provides a quantitative description of electrochemical reversibility ${ }^{77}$ to unveil the nature of a donor-acceptor interaction ${ }^{87}$. Specifically, based on the Marcus theory ${ }^{88}$ that specifies that the electron transfer provides valuable basis for quantitatively comparing homogeneous and heterogeneous processes ${ }^{89}$, with the theory assuming that after the reaction reactants are weakly coupled and stay individually rather than form a complex. Such processes could potentially help identify whether the binding affinity of the kinesin-FCA to the MT was changed due to the kinesin labeling with FCA, since previous analysis (Randles-Sevcik Equation $)^{90}$ has showed that the adsorption-controlled redox process reveals a linear relationship between the peak current and the scan rate while the diffusion-controlled redox process showed a linear relationship between the peak current and the square root scan rate. Kinesin-FCA conjugate with a concentration smaller than the saturated concentration (42.2 $\mathrm{nM}$ ) was used to form kinesin-FCA-MT assembly on the electrode surface in order to reduce any embedment.

As shown in Figure 4a, a pair of well-defined redox peak appeared when the scan rate was lower than $300 \mathrm{mV} / \mathrm{s}$, i.e., from 6 to $300 \mathrm{mV} / \mathrm{s}$. However, when the scan rate was faster (larger than $300 \mathrm{mV} / \mathrm{s}$ ), the redox peaks were hardly defined, as the change in current was too large to be recorded. Complementary, when the scan rate decreased to $6 \mathrm{mV}$, both the currents of anodic and cathodic peaks $\left(\mathrm{I}_{\mathrm{pa}}\right.$ and $\left.\mathrm{I}_{\mathrm{pc}}\right)$ increased. Further, the anodic potential $\left(\mathrm{E}_{\mathrm{pa}}\right)$ shifted to became more negative while the cathodic potential $\left(\mathrm{E}_{\mathrm{pc}}\right)$ became more positive when the $\Delta \mathrm{E}_{\mathrm{p}}$ increased from $39 \mathrm{mV}$ to $363 \mathrm{mV}$. Our experiments are supported by the Laviron's Equation $^{91}$ which showed the dependence of the anodic/cathodic potential on the scan rate for such a quasi-reversible reaction. Herein the reaction is being considered quasi-reversible since the peak separation is large than $59 \mathrm{mV}$ however only slightly. Briefly, for the quasi-reversible reaction, the anodic and cathodic potentials becomes larger (anodic) or smaller (cathodic) with the increasing of the scan rate.

Considering the electrochemical probes as being the immobilized FCA-kinesin-MT assembly and considering that the redox reaction is an adsorption-controlled electrochemical process, a direct relationship between the peak currents and the scan rates was also obtained (Figure 4b). Analysis showed that the peak current $\left(\mathrm{I}_{\mathrm{p}}\right)$ is proportional to the scan rates confirming that the absorbed species or the material at the surface of the electrode are the 
ones mainly contributing to the observed reaction ${ }^{92}$. Our analysis are supported by previous studies that showed that the adsorbed bromothymol blue for instance had an adsorption-control redox process onto a carbon paste electrode ${ }^{93}$.

The peak separation at the highest scan rate $(300 \mathrm{mV} / \mathrm{s})$ was further used to estimate the heterogeneous electron transfer rate constant. Such rate constant allows depicting the rate at which an electron can move or jump from the electron donor to the electron acceptor ${ }^{94}$. For this, the electron transfer coefficient $\mathrm{k}$ and transfer coefficient $\alpha$ were calculated from the Laviron's equation by using:

$$
E_{p c}=E^{o^{\prime}}-\frac{R T}{\alpha n F} \ln \left(\frac{\alpha n F v}{R T k}\right)
$$

or

$$
E_{p a}=E^{o^{\prime}}-\frac{R T}{(1-\alpha) \mathrm{nF}} \ln \left(\frac{(1-\alpha) \mathrm{nFv}}{R T k}\right)
$$

where $\mathrm{E}_{\mathrm{pc}}$ and $\mathrm{E}_{\mathrm{pa}}$ are the cathodic and anodic peak potentials and the $\mathrm{E}^{\mathrm{o}^{\prime}}$ is the formal potential respectively. The $\mathrm{E}^{\mathrm{o}^{\prime}}$ was calculated from the $\mathrm{E}_{\mathrm{p}} \mathrm{vs} . \mathrm{v}$ plot by extrapolating the line to $\mathrm{v}=0^{95}$ and considering that $\mathrm{n}$ is the number of electrons being transferred at the conductive surface, $\alpha$ is the transfer coefficient for the catholic process, $\mathrm{R}$ is the gas constant and $\mathrm{T}$ is the temperature respectively. The Laviron's equation assumes both redox states were strongly adsorbed and non-interacting on the surface, while the adsorption and electron-transfer processes involving solution-resident species would not affect measured currents ${ }^{96}$.

Figure 4c shows the Laviron's plots and allowed the electron transfer rate constant $\mathrm{k}$ to be calculated as being $0.23 \mathrm{~s}^{-1}$. Our result is supported by previous research that showed the electron transfer rate constant was $11.80 \mathrm{~s}^{-1}$ between FCA and cysteamine modified gold electrode and $0.27 \mathrm{~s}^{-1}$ between FCA and cysteamine/single wall carbon nanotube modified gold electrode ${ }^{97}$ respectively. Our result also reflected a slow heterogeneous electron transfer at the interface (since the value is small) that could be caused by the conformational changes in FCA-kinesin-MT assembly or the embedment of the FCA molecules at such interfaces ${ }^{98,99}$. Briefly, if the FCA was embedded then the hindered electron transfer between the FCA and the electrode surface will limit transfer at the interface being recorded ${ }^{74}$. Such embedment could be a result of kinesin assuming different conformation at the MT interfaces; previous studies have showed that kinesin could assume either a stand up or a lay on geometry ${ }^{100}$ which could lead to further hindering. 

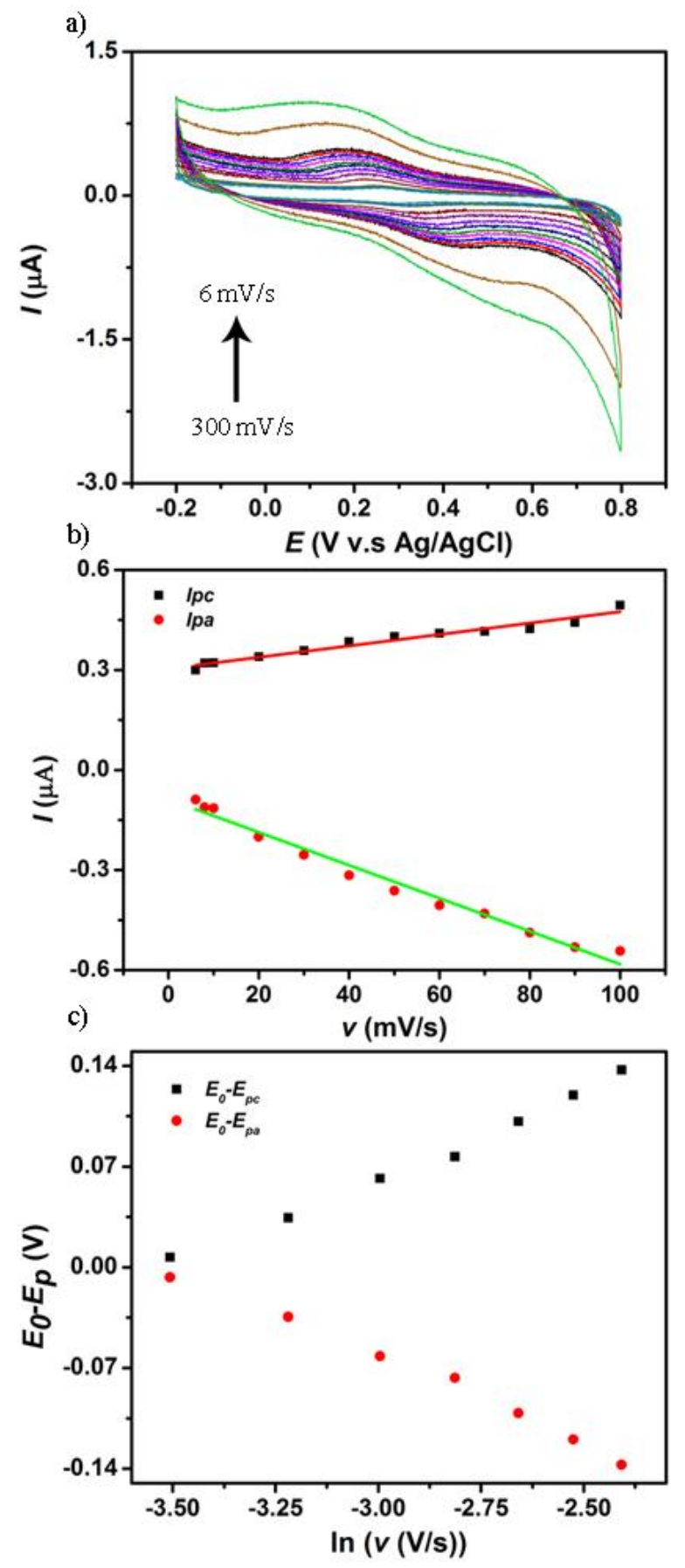

Figure 4: a) CV of FCA-kinesin at modified electrode under different scan rates (6-300 $\mathrm{mV} / \mathrm{s}$ ). The concentration of the FCA-kinesin was kept constant at $42.2 \mathrm{nM}$, which represented that concentration that limits the saturation sites of the MTs. b) The linear relationship between the scan rates and the peak currents of the FCA-kinesin conjugates. c) Laviron's plots. All experiments were carried at modified electrode (Au/MUA/Anti-tubulin Antibody (BSA)/MT) in BRB80 buffer (pH 7.1) with $10 \mu \mathrm{M}$ taxol. 
Upon confirming the electron transfer at the FCA-kinesin-MT assembly, we further evaluated the ability for detection of FCA-kinesin conjugates dissociation. It is hypothesized herein that if the ability for detection of the dissociation of FCA-kinesin conjugates from MT could be recorded, such strategy will help define and characterize the proposed biosensor's sensitivity of kinesin-FCA unbinding, with the sensitivity being defined as the ability of the sensor to provide a signal at a threshold concentration. Association and dissociation processes of kinesin-MTs are known to occur naturally in the cell and were shown to be responsible for functionally modulating cellular activities from DNA repair to kinesin-MT binding events involved in the cell division process ${ }^{101,102}$. Impediment of such processes was shown to lead to several diseases such as amyotrophic lateral sclerosis (ALS) ${ }^{103}$ and Alzheimer ${ }^{104}$. Further, impeded association or dissociation has also been shown to affect cellular activity or cell division ${ }^{105}$. When mimicked in synthetic environment, impeded association or dissociation of kinesin-MT conjugates were shown to lead to failure of a synthetic biosensor ${ }^{106}$. Sensitivity of dissociation was evaluated considering the performance and functionality of kinesin molecules. Specifically, in the cells kinesin 1 performs processive steps of $8 \mathrm{~nm}^{107}$ under the chemical energy of ATP. Previous research has shown that kinesin-MT motion is influenced by the ATP concentration available for the individual mechanical cycle performed by kinesin, with analysis showing that the velocity of a single molecule moving along the MT is obeying the Michaelis-Menten kinetics ${ }^{108}$. Previous research has also showed that using the chemical energy of ATP and transforming it into mechanical cycle ${ }^{42}$ will lead to kinesin releasing the MT binding sites in a bipedal stepping-like manner and at a controlled velocity ${ }^{109}$.

The schematic of FCA-kinesin conjugates dissociation as triggered by the addition of ATP (constant concentration of $250 \mu \mathrm{M}$ ) is shown in Figure 5a with specific analysis reflected in Figure 5b. As revealed, the electrochemical signal at the electrode interface decreased upon addion of ATP presumably as a result of kinesin molecules stepping out of the immobilized MT, releasing their binding sites and thus leading to decreased of individual FCA-kinesin conjugates bound at one time onto the modified electrode and capable to undergo redox reactions at such interface. Herein in order to record maximum dissociation sensitivity we used the kinesin at the saturated concentration as identified in Figure 3a, i.e., $84.3 \mathrm{nM}$. Specifically, analysis performed for $20 \mathrm{CV}$ cycles with scanning being stopped for $20 \mathrm{sec}$ after finishing each such CV cycle, showed that the peak currents decreased ${ }^{110}$ with the redox peak current of the FCA-kinesin being about $1.332 \mu \mathrm{A}$ and $-1.726 \mu \mathrm{A}$ respectively at the first $\mathrm{CV}$ cycle, and $0.687 \mu \mathrm{A}$ and $-0.948 \mu \mathrm{A}$ after the last $20^{\text {th }}$ cycle. 
Based on these observations and the theoretical concentration of FCA-kinesin conjugates used herein and known to lead to full MT coverage, the numbers of FCA-kinesin conjugates dissociating during the cyclic processes was estimated as being about $1.2 \times 10^{16}$ FCA-kinesin conjugates onto the MT in cycle 1 and $7.0 \times 10^{15}$ in cycle 20 . Based on such analysis, about $3 \times 10^{15}$ FCA-kinesin dissociated from the MT in the 20 cycles being considered.

Figure 5c shows the relationship between the number of FCA-kinesin conjugates left at a given time onto the MT and time. As shown, the number of the FCA-kinesin conjugates associated with the MT decreased rapidly at the beginning of the scanning (i.e., during the 0 to the $150 \mathrm{~s}$ time interval), to subsequently become stable (i.e., during the 150 to $800 \mathrm{~s}$ time interval). Also according to Figure 5c, about $2.6 \times 10^{12}$ FCA-kinesin conjugates dissociated from the MT per second ${ }^{110,111}$. The resulting sensitivity of dissociation was thus $2.04 \times 10^{12}$ molecules $\left(3.39 \times 10^{-10}\right.$ mol considering that kinesin molecular weight is $188 \mathrm{kDa}^{29}$ and the Avogadro number is $6.0221409 \times 10^{2}$ ).

This is the first demonstration of dissociation events being recorded at the level of single molecule binding as dictated by the recognition events between kinesin and MT. Further, this is the first demonstration to allow characterization of the event under chemical (energetical) transformation as associated with kinesin stepping onto the MT. By precisely controlling of the amount of ATP used in the system, we foresee control of the number of the FCA-kinesin conjugates left on the MT, again, at a single molecule level. Further, by integration of such biosensor on a lab-on-chip platform, we foresee the formation of a "nanoswitch" ("on" to be provided by the ATP while the "off" is to be provided by the AMP-PNP in solution) for applications ranging from biosensing, to logic gate design and operation, and diagnostics. In addition, we also foresee realization of storage and controlled release capabilities based on kinesin carried binding and unbinding of different cargo to be applicable to technologies from drug delivery to corrosion inhibition. 
a)
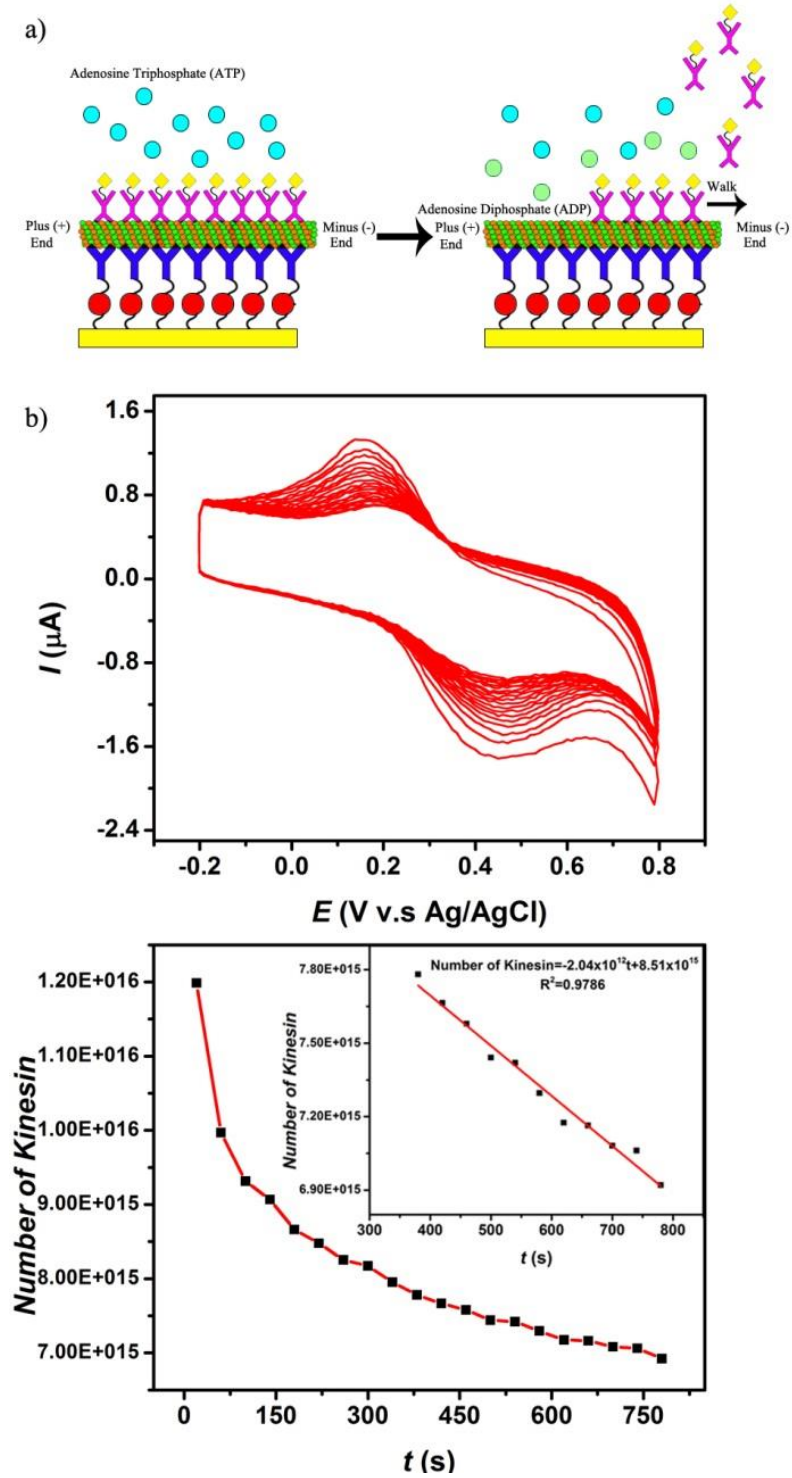

Figure 5: a) Schematic illustration of the FCA-kinesin conjugates stepping of the MT imoobilized at the electrode interface upon the addition of ATP. b) CV of FCA-kinesin conjugate at the modified electrode modified electrode (Au/MUA/Anti-tubulin Antibody/MT) after adding $250 \mu \mathrm{M}$ ATP. 


\section{Conclusions}

Herein we demonstrated successful design and fabrication of a biosensor based on kinesin-MT system; the designed biosensor was capable to allow detection of association and dissociation events based on redox reactions at user-designed electrode interfaces. Our biosensor functionality is inspired by cellular processes driven by molecular motors and could be implemented as a versatile nanoswitch under different energy sources. By integrating such a biosensor into a microfluidic chip, applications in biomedical, forensic and environmental analysis are foreseen. 


\section{References:}

1 Long, F., Zhu, A. \& Shi, H. Recent advances in optical biosensors for environmental monitoring and early warning. Sensors 13, 13928-13948 (2013).

2 Hayat, A. \& Marty, J. L. Disposable screen printed electrochemical sensors: Tools for environmental monitoring. Sensors 14, 10432-10453 (2014).

3 Kang, D. et al. DNA biomolecular-electronic encoder and decoder devices constructed by multiplex biosensors. NPG Asia Materials 4, e1 (2012).

4 Turner, A. P. Biosensors: sense and sensibility. Chemical Society Reviews 42, 3184-3196 (2013).

5 Gamella, M. et al. Integrated multienzyme electrochemical biosensors for monitoring malolactic fermentation in wines. Talanta 81, 925-933 (2010).

6 Akin, M. et al. A new set up for multi-analyte sensing: At-line bio-process monitoring. Biosensors and Bioelectronics 26, 4532-4537 (2011).

7 Huang, J. et al. Pyrene - excimer probes based on the hybridization chain reaction for the detection of nucleic acids in complex biological fluids. Angewandte Chemie International Edition 50, 401-404 (2011).

8 Mehrotra, P. Biosensors and their applications-A review. Journal of oral biology and craniofacial research 6, 153-159 (2016).

9 Jung, J. H., Cheon, D. S., Liu, F., Lee, K. B. \& Seo, T. S. A graphene oxide based immuno - biosensor for pathogen detection. Angewandte Chemie International Edition 49, 5708-5711 (2010).

10 Abuzairi, T. et al. Maskless functionalization of a carbon nanotube dot array biosensor using an ultrafine atmospheric pressure plasma jet. Carbon 89, 208-216 (2015).

11 Deng, Y., Wang, W., Ma, C. \& Li, Z. Fabrication of an electrochemical biosensor array for simultaneous detection of L-glutamate and acetylcholine. Journal of biomedical nanotechnology 9, 1378-1382 (2013).

12 Pollet, J. et al. Fast and accurate peanut allergen detection with nanobead enhanced optical fiber SPR biosensor. Talanta 83, 1436-1441 (2011).

13 Zhou, C. et al. Development of a fast and sensitive glucose biosensor using iridium complex-doped electrospun optical fibrous membrane. Analytical chemistry 85, 1171-1176 (2012). 
14 Claes, T., Bogaerts, W. \& Bienstman, P. Vernier-cascade label-free biosensor with integrated arrayed waveguide grating for wavelength interrogation with low-cost broadband source. Optics letters 36, 3320-3322 (2011).

15 Zhang, B. \& Cui, T. An ultrasensitive and low-cost graphene sensor based on layer-by-layer nano self-assembly. Applied Physics Letters 98, 073116 (2011).

16 Sarkar, D. \& Banerjee, K. Proposal for tunnel-field-effect-transistor as ultra-sensitive and label-free biosensors. Applied Physics Letters 100, 143108 (2012).

17 Shen, M.-Y., Li, B.-R. \& Li, Y.-K. Silicon nanowire field-effect-transistor based biosensors: From sensitive to ultra-sensitive. Biosensors and Bioelectronics $6 \mathbf{0}$, 101-111 (2014).

18 Choi, S. \& Chae, J. Microfluidic Biosensors for Thyroglobulin Detection and Application to Thyroid Cancer. Biosensors and Cancer, 300 (2012).

19 Chen, X., Wang, W. \& Wang, J. A DNA assay protocol in a lab-on-valve meso-fluidic system with detection by laser-induced fluorescence. Analyst 130, 1240-1244 (2005).

20 Cho, I.-H. \& Irudayaraj, J. In-situ immuno-gold nanoparticle network ELISA biosensors for pathogen detection. International journal of food microbiology 164, 70-75 (2013).

21 Zhao, Z. et al. Nuclease-free target recycling signal amplification for ultrasensitive multiplexing DNA biosensing. Biosensors and Bioelectronics 94, 605-608, doi:https://doi.org/10.1016/j.bios.2017.03.051 (2017).

22 Nasirizadeh, N., Zare, H. R., Pournaghi-Azar, M. H. \& Hejazi, M. S. Introduction of hematoxylin as an electroactive label for DNA biosensors and its employment in detection of target DNA sequence and single-base mismatch in human papilloma virus corresponding to oligonucleotide. Biosensors and Bioelectronics 26, 2638-2644 (2011).

23 Sun, X. \& Wang, X. Acetylcholinesterase biosensor based on prussian blue-modified electrode for detecting organophosphorous pesticides. Biosensors and Bioelectronics 25, 2611-2614, doi:https://doi.org/10.1016/j.bios.2010.04.028 (2010).

24 Kumar, S. et al. Reduced graphene oxide modified smart conducting paper for cancer biosensor. Biosensors and Bioelectronics 73, 114-122 (2015).

25 Balasubramanian, S., Sorokulova, I. B., Vodyanoy, V. J. \& Simonian, A. L. Lytic phage as a specific and selective probe for detection of Staphylococcus aureus-A 
surface plasmon resonance spectroscopic study. Biosensors and Bioelectronics 22, 948-955 (2007).

26 Gyawali, P. Detection of Viable Hookworm Ova from Wastewater and Sludge. (2017).

27 Van Dorst, B. et al. Recent advances in recognition elements of food and environmental biosensors: a review. Biosensors and Bioelectronics 26, 1178-1194 (2010).

28 Harris, J. M., Reyes, C. \& Lopez, G. P. Common causes of glucose oxidase instability in in vivo biosensing: a brief review. Journal of diabetes science and technology 7, 1030-1038, doi:10.1177/193229681300700428 (2013).

29 Cyr, J. L., Pfister, K. K., Bloom, G. S., Slaughter, C. A. \& Brady, S. T. Molecular genetics of kinesin light chains: generation of isoforms by alternative splicing. Proceedings of the National Academy of Sciences 88, 10114-10118 (1991).

30 Kapitein, L. C. \& Hoogenraad, C. C. Building the neuronal microtubule cytoskeleton. Neuron 87, 492-506 (2015).

31 Yang, H., Ganguly, A. \& Cabral, F. Inhibition of cell migration and cell division correlate with distinct effects of microtubule inhibiting drugs. Journal of biological chemistry, jbc. M110. 160820 (2010).

32 Kevenaar, J. T. et al. Kinesin-binding protein controls microtubule dynamics and cargo trafficking by regulating kinesin motor activity. Current Biology 26, 849-861 (2016).

33 Fischer, T., Agarwal, A. \& Hess, H. A smart dust biosensor powered by kinesin motors. Nature nanotechnology 4, 162 (2009).

34 Won, E.-J. et al. Response of glutathione S-transferase (GST) genes to cadmium exposure in the marine pollution indicator worm, Perinereis nuntia. Comparative Biochemistry and Physiology Part C: Toxicology \& Pharmacology 154, 82-92 (2011).

35 Soto, C. M., Martin, B. D., Sapsford, K. E., Blum, A. S. \& Ratna, B. R. Toward single molecule detection of staphylococcal enterotoxin B: mobile sandwich immunoassay on gliding microtubules. Analytical chemistry 80, 5433-5440 (2008).

36 Bachand, M., Bachand, G. D., Greene, A. C. \& Carroll-Portillo, A. In vivo collection of rare proteins using kinesin-based" nano-harvesters". (Sandia National Laboratories, 2008). 
37 Gan, W. Q., Man, S., Senthilselvan, A. \& Sin, D. Association between chronic obstructive pulmonary disease and systemic inflammation: a systematic review and a meta-analysis. Thorax 59, 574-580 (2004).

$38 \mathrm{Hu}, \mathrm{X}$. et al. Biological self-assembly and recognition used to synthesize and surface guide next generation of hybrid materials. ACS applied materials \& interfaces 10, 28372-28381 (2018).

39 Hu, X., Dong, C., Su, R., Xu, Q. \& Dinu, C. Z. Protein self-assembly onto nanodots leads to formation of conductive bio-based hybrids. Scientific reports 6, 38252 (2016).

40 Landers, T. A., Greenberg, H. B. \& Robinson, W. S. Structure of hepatitis B Dane particle DNA and nature of the endogenous DNA polymerase reaction. Journal of virology 23, 368-376 (1977).

41 Fernández, L. \& Carrero, H. Electrochemical evaluation of ferrocene carboxylic acids confined on surfactant-clay modified glassy carbon electrodes: oxidation of ascorbic acid and uric acid. Electrochimica Acta 50, 1233-1240 (2005).

42 Dong, C. \& Dinu, C. Z. Molecular trucks and complementary tracks for bionanotechnological applications. Current opinion in biotechnology 24, 612-619 (2013).

43 Dinu, C. Z. et al. Parallel Manipulation of Bifunctional DNA Molecules on Structured Surfaces Using Kinesin - Driven Microtubules. Small 2, 1090-1098 (2006).

$44 \mathrm{Hu}, \mathrm{X}$. \& Dinu, C. Z. Analysis of affinities between specific biological ligands using atomic force microscopy. Analyst 140, 8118-8126 (2015).

45 Kull, F. J., Sablin, E. P., Lau, R., Fletterick, R. J. \& Vale, R. D. Crystal structure of the kinesin motor domain reveals a structural similarity to myosin. Nature 380, 550 (1996).

46 Raab, M. \& Hancock, W. O. Transport and detection of unlabeled nucleotide targets by microtubules functionalized with molecular beacons. Biotechnology and bioengineering 99, 764-773 (2008).

47 Sardar, H. S. \& Gilbert, S. P. Microtubule Capture by Mitotic Kinesin Centromere Protein E (CENP-E). The Journal of Biological Chemistry 287, 24894-24904, doi:10.1074/jbc.M112.376830 (2012). 
48 Ramachandran, S., Ernst, K.-H., Bachand, G. D., Vogel, V. \& Hess, H. Selective Loading of Kinesin-Powered Molecular Shuttles with Protein Cargo and its Application to Biosensing. Small 2, 330-334, doi:10.1002/smll.200500265 (2006).

49 Stender, A. S. et al. Single Cell Optical Imaging and Spectroscopy. Chemical reviews 113, 2469-2527, doi:10.1021/cr300336e (2013).

50 Fischer, T., Agarwal, A. \& Hess, H. A smart dust biosensor powered by kinesin motors. Nat Nano 4, 162-166 (2009).

51 Ramachandran, S., Ernst, K. H., Bachand, G. D., Vogel, V. \& Hess, H. Selective Loading of Kinesin - Powered Molecular Shuttles with Protein Cargo and its Application to Biosensing. Small 2, 330-334 (2006).

52 Lim, Y. C., Kouzani, A. Z. \& Duan, W. Lab-on-a-chip: a component view. Microsystem Technologies 16, 1995-2015, doi:10.1007/s00542-010-1141-6 (2010).

53 Pires, N. M. M., Dong, T., Hanke, U. \& Hoivik, N. Recent developments in optical detection technologies in lab-on-a-chip devices for biosensing applications. Sensors 14, 15458-15479 (2014).

54 Nimse, S. B. \& Kim, T. Biological applications of functionalized calixarenes. Chemical Society Reviews 42, 366-386, doi:10.1039/C2CS35233H (2013).

55 Ge, D. \& Levicky, R. A Comparison of Five Bioconjugatable Ferrocenes for Labeling of Biomolecules. Chemical communications (Cambridge, England) 46, 7190-7192, doi:10.1039/c0cc02044c (2010).

56 Yang, N., Wei, Y., Kang, X. \& Su, Z. Selective recognition and sensing for adenosine triphosphate by label-free electrochemistry based on its inclusion with per-6-ammonium-[small beta]-cyclodextrin. Analytical Methods 7, 10129-10135, doi:10.1039/C5AY01264C (2015).

57 Sharan, R. et al. Conserved patterns of protein interaction in multiple species. Proceedings of the National Academy of Sciences of the United States of America 102, 1974-1979, doi:10.1073/pnas.0409522102 (2005).

58 Hunter, A. W. \& Wordeman, L. How motor proteins influence microtubule polymerization dynamics. Journal of cell science 113, 4379-4389 (2000).

59 Kawaguchi, K. \& Ishiwata, S. i. Nucleotide-Dependent Single- to Double-Headed Binding of Kinesin. Science 291, 667-669, doi:10.1126/science.291.5504.667 (2001). 
60 Schaap, Iwan A. T., Carrasco, C., de Pablo, Pedro J. \& Schmidt, Christoph F. Kinesin Walks the Line: Single Motors Observed by Atomic Force Microscopy. Biophysical Journal 100, 2450-2456 (2011).

61 Thébault, P., Boujday, S., Sénéchal, H. \& Pradier, C.-M. Investigation of an Allergen Adsorption on Amine- and Acid-Terminated Thiol Layers: Influence on Their Affinity to Specific Antibodies. The Journal of Physical Chemistry B 114, 10612-10619, doi:10.1021/jp103164b (2010).

62 Ciracì, C. et al. Probing the ultimate limits of plasmonic enhancement. Science 337, 1072-1074 (2012).

63 Kim, C. A. \& Bowie, J. U. SAM domains: uniform structure, diversity of function. Trends in biochemical sciences 28, 625-628 (2003).

64 Masson, J.-F. et al. Reduction of nonspecific protein binding on surface plasmon resonance biosensors. Analytical and Bioanalytical Chemistry 386, 1951-1959, doi:10.1007/s00216-006-0834-2 (2006).

65 Lisdat, F. \& Schäfer, D. The use of electrochemical impedance spectroscopy for biosensing. Analytical and Bioanalytical Chemistry 391, 1555, doi:10.1007/s00216-008-1970-7 (2008).

66 Dai, Z., Hu, X., Wu, H. \& Zou, X. A label-free electrochemical assay for quantification of gene-specific methylation in a nucleic acid sequence. Chemical Communications 48, 1769-1771 (2012).

67 Siebert, E., Hammouche, A. \& Kleitz, M. Impedance spectroscopy analysis of La1 xSritxMnO3-yttria-stabilized zirconia electrode kinetics. Electrochimica Acta 40, 1741-1753, (1995).

68 Karazehir, T., Ates, M. \& Sarac, A. S. Mott-Schottky and morphologic analysis of poly (pyrrole-n-propionic acid) in various electrolyte systems. International Journal of Electrochemical Science 10, 6146-6163 (2015).

69 Che, G., Lakshmi, B. B., Fisher, E. R. \& Martin, C. R. Carbon nanotubule membranes for electrochemical energy storage and production. Nature 393, 346-349 (1998).

70 Padeste, C., Grubelnik, A. \& Tiefenauer, L. Ferrocene-avidin conjugates for bioelectrochemical applications. Biosensors and Bioelectronics 15, 431-438 (2000).

71 Murthy, A. S. N. \& Sharma, J. Glucose oxidase bound to self-assembled monolayers of bis(4-pyridyl) disulfide at a gold electrode: Amperometric determination of glucose. Analytica Chimica Acta 363, 215-220 (1998). 
72 Pandurangachar, M. et al. Electrochemical investigations of potassium ferricyanide and dopamine by 1-butyl-4-methylpyridinium tetrafluoro borate modified carbon paste electrode: A cyclic voltammetric study. Int J Electrochem Sci 5, 1187-1202 (2010).

73 Wu, H., Wang, P., Hu, X., Dai, Z. \& Zou, X. Site-selective probe for investigating the asynchronous unfolding of domains in bovine serum albumin. Talanta 84, 881-886 (2011).

74 Schumacher, S., Nagel, T., Scheller, F. W. \& Gajovic-Eichelmann, N. Alizarin Red S as an electrochemical indicator for saccharide recognition. Electrochimica Acta 56, 6607-6611 (2011).

75 Nugent, J., Santhanam, K., Rubio, A. \& Ajayan, P. Fast electron transfer kinetics on multiwalled carbon nanotube microbundle electrodes. Nano letters 1, 87-91 (2001).

76 Chen, P., Fryling, M. A. \& McCreery, R. L. Electron Transfer Kinetics at Modified Carbon Electrode Surfaces: The Role of Specific Surface Sites. Analytical Chemistry 67, 3115-3122, doi:10.1021/ac00114a004 (1995).

77 Klingler, R. \& Kochi, J. Electron-transfer kinetics from cyclic voltammetry. Quantitative description of electrochemical reversibility. The Journal of Physical Chemistry 85, 1731-1741 (1981).

78 Akiyoshi, B. et al. Tension directly stabilizes reconstituted kinetochore-microtubule attachments. Nature 468, 576 (2010).

79 Kozielski, F. et al. The crystal structure of dimeric kinesin and implications for microtubule-dependent motility. Cell 91, 985-994 (1997).

80 Zhang, J. et al. Nanorod-constructed porous Co $3 \mathrm{O} 4$ nanowires: highly sensitive sensors for the detection of hydrazine. Analyst 140, 1686-1692 (2015).

81 Giancoli, D. C. Physics for scientists and engineers. Vol. 3 (Prentice hall Upper Saddle River, NJ, 2000).

82 Letelier, M. E., Sánchez-Jofré, S., Peredo-Silva, L., Cortés-Troncoso, J. \& Aracena-Parks, P. Mechanisms underlying iron and copper ions toxicity in biological systems: Pro-oxidant activity and protein-binding effects. Chemico-biological interactions 188, 220-227 (2010).

83 Auton, M., Holthauzen, L. M. F. \& Bolen, D. W. Anatomy of energetic changes accompanying urea-induced protein denaturation. Proceedings of the National Academy of Sciences 104, 15317-15322 (2007). 
84 Wang, S.-F., Chen, T., Zhang, Z.-L., Pang, D.-W. \& Wong, K.-Y. Effects of hydrophilic room-temperature ionic liquid 1-butyl-3-methylimidazolium tetrafluoroborate on direct electrochemistry and bioelectrocatalysis of heme proteins entrapped in agarose hydrogel films. Electrochemistry communications 9, 1709-1714 (2007).

85 Wei, S., Dandan, W., Ruifang, G. \& Kui, J. Direct electrochemistry and electrocatalysis of hemoglobin in sodium alginate film on a BMIMPF 6 modified carbon paste electrode. Electrochemistry Communications 9, 1159-1164 (2007).

86 Yu, H.-Z., Luo, C.-Y., Sankar, C. G. \& Sen, D. Voltammetric procedure for examining DNA-modified surfaces: quantitation, cationic binding activity, and electron-transfer kinetics. Analytical chemistry 75, 3902-3907 (2003).

87 Newton, M. D. Quantum chemical probes of electron-transfer kinetics: the nature of donor-acceptor interactions. Chemical Reviews 91, 767-792 (1991).

88 Marcus, R. On the theory of oxidation-reduction reactions involving electron transfer. v. comparison and properties of electrochemical and chemical rate constants1. The Journal of Physical Chemistry 67, 853-857 (1963).

89 Mohilner, D. M. Double-layer effects in the kinetics of heterogeneous electron exchange reactions. The Journal of Physical Chemistry 73, 2652-2662 (1969).

90 Jain, R., Gupta, V. K., Jadon, N. \& Radhapyari, K. Voltammetric determination of cefixime in pharmaceuticals and biological fluids. Analytical Biochemistry 407, 79-88 (2010).

91 Laviron, E. Adsorption, autoinhibition and autocatalysis in polarography and in linear potential sweep voltammetry. Journal of Electroanalytical Chemistry and Interfacial Electrochemistry 52, 355-393 (1974).

$92 \mathrm{Wu}$, J.-F., Xu, M.-Q. \& Zhao, G.-C. Graphene-based modified electrode for the direct electron transfer of Cytochrome c and biosensing. Electrochemistry Communications 12, 175-177 (2010).

93 Chandrashekar, B., Swamy, B. K., Mahesh, K. V., Chandra, U. \& Sherigara, B. Electrochemical studies of bromothymol blue at surfactant modified carbon paste electrode by using cyclic voltammetry. Int. J. Electrochem. Sci 4, 471-480 (2009).

94 Wipf, D. O., Kristensen, E. W., Deakin, M. R. \& Wightman, R. M. Fast-scan cyclic voltammetry as a method to measure rapid heterogeneous electron-transfer kinetics. Analytical Chemistry 60, 306-310 (1988). 
95 Fotouhi, L., Fatollahzadeh, M. \& Heravi, M. M. Electrochemical behavior and voltammetric determination of sulfaguanidine at a glassy carbon electrode modified with a multi-walled carbon nanotube. Int. J. Electrochem. Sci 7, 3919-3928 (2012).

96 Reeves, J. H., Song, S. \& Bowden, E. F. Application of square wave voltammetry to strongly adsorbed quasireversible redox molecules. Analytical Chemistry 65, 683-688 (1993).

97 Nkosi, D., Pillay, J., Ozoemena, K. I., Nouneh, K. \& Oyama, M. Heterogeneous electron transfer kinetics and electrocatalytic behaviour of mixed self-assembled ferrocenes and SWCNT layers. Physical Chemistry Chemical Physics 12, 604-613, doi:10.1039/B918754E (2010).

98 Brunschwig, B. S. \& Sutin, N. Directional electron transfer: Conformational interconversions and their effects on observed electron-transfer rate constants. Journal of the American Chemical Society 111, 7454-7465 (1989).

99 Grabowski, Z. R., Rotkiewicz, K. \& Rettig, W. Structural changes accompanying intramolecular electron transfer: focus on twisted intramolecular charge-transfer states and structures. Chemical reviews 103, 3899-4032 (2003).

100 Jung, Y., Jeong, J. Y. \& Chung, B. H. Recent advances in immobilization methods of antibodies on solid supports. Analyst 133, 697-701, doi:10.1039/B800014J (2008).

101 Seeger, M. A. \& Rice, S. E. Microtubule-associated Protein-like Binding of the Kinesin-1 Tail to Microtubules. Journal of Biological Chemistry 285, 8155-8162, doi:10.1074/jbc.M109.068247 (2010).

102 Barlan, K., Lu, W. \& Gelfand, V. I. The microtubule-binding protein ensconsin is an essential cofactor of kinesin-1. Current biology : $C B$ 23, 317-322, doi:10.1016/j.cub.2013.01.008 (2013).

103 Mandelkow, E. \& Mandelkow, E.-M. Kinesin motors and disease. Trends in Cell Biology 12, 585-591 (2002).

104 Goldstein, L. S. B. Kinesin molecular motors: Transport pathways, receptors, and human disease. Proceedings of the National Academy of Sciences of the United States of America 98, 6999-7003, doi:10.1073/pnas.111145298 (2001).

105 Altieri, D. C. Survivin, versatile modulation of cell division and apoptosis in cancer. Oncogene 22, 8581-8589 (2003).

106 Clemmens, J. et al. Motor-protein "roundabouts": microtubules moving on kinesin-coated tracks through engineered networks. Lab on a Chip 4, 83-86 (2004). 
107 Jamison, D. K., Driver, J. W., Rogers, A. R., Constantinou, P. E. \& Diehl, M. R. Two kinesins transport cargo primarily via the action of one motor: implications for intracellular transport. Biophysical journal 99, 2967-2977 (2010).

108 Schnitzer, M. J., Visscher, K. \& Block, S. M. Force production by single kinesin motors. Nature cell biology 2, 718-723 (2000).

109 Svoboda, K. \& Block, S. M. Force and velocity measured for single kinesin molecules. Cell 77, 773-784 (1994).

110 Subramanian, R., Ti, S.-C., Tan, L., Darst, S. A. \& Kapoor, T. M. Marking and measuring single microtubules by PRC1 and kinesin-4. Cell 154, 377-390 (2013).

111 Olsen, T. \& Thygesen, K. S. Random phase approximation applied to solids, molecules, and graphene-metal interfaces: From van der Waals to covalent bonding. Physical Review B 87, 075111 (2013). 


\section{Supporting Information}

1. The sequence of kinesin 1 (simply called kinesin) heavy chain

This sequence was imported from previous research ${ }^{1}$.

$\begin{array}{lllll}10 & 20 & 30 & 40 & 50\end{array}$

MADLAECNIK VMCRFRPLNE SEVNRGDKYI AKFQGEDTVV IASKPYAFDR

$\begin{array}{lllll}60 & 70 & 80 & 90 & 100\end{array}$

VFQSSTSQEQ VYNDCAKKIV KDVLEGYNGT IFAYGQTSSG KTHTMEGKLH

$\begin{array}{lllll}110 & 120 & 130 & 140 & 150\end{array}$

DPEGMGIIPR IVQDIFNYIY SMDENLEFHI KVSYFEIYLD KIRDLLDVSK

$\begin{array}{lllll}160 & 170 & 180 & 190 & 200\end{array}$

TNLSVHEDKN RVPYVKGCTE RFVCSPDEVM DTIDEGKSNR HVAVTNMNEH

$\begin{array}{lllll}210 & 220 & 230 & 240 & 250\end{array}$

SSRSHSIFLI NVKQENTQTE QKLSGKLYLV DLAGSEKVSK TGAEGAVLDE

$\begin{array}{lllll}260 & 270 & 280 & 290 & 300\end{array}$

AKNINKSLSA LGNVISALAE GSTYVPYRDS KMTRILQDSL GGNCRTTIVI

$\begin{array}{lllll}310 & 320 & 330 & 340 & 350\end{array}$

CCSPSSYNES ETKSTLLFGQ RAKTIKNTVC VNVELTAEQW KKKYEKEKEK

$\begin{array}{lllll}360 & 370 & 380 & 390 & 400\end{array}$

NKILRNTIQW LENELNRWRN GETVPIDEQF DKEKANLEAF TVDKDITLTN

$\begin{array}{lllll}410 & 420 & 430 & 440 & 450\end{array}$

DKPATAIGVI GNFTDAERRK CEEEIAKLYK QLDDKDEEIN QQSQLVEKLK

$\begin{array}{lllll}460 & 470 & 480 & 490 & 500\end{array}$

TQMLDQEELL ASTRRDQDNM QAELNRLQAE NDASKEEVKE VLQALEELAV

$\begin{array}{lllll}510 & 520 & 530 & 540 & 550\end{array}$

NYDQKSQEVE DKTKEYELLS DELNQKSATL ASIDAELQKL KEMTNHQKKR

$\begin{array}{lllll}560 & 570 & 580 & 590 & 600\end{array}$

AAEMMASLLK DLAEIGIAVG NNDVKQPEGT GMIDEEFTVA RLYISKMKSE
610
620
630
640
650

VKTMVKRCKQ LESTQTESNK KMEENEKELA ACQLRISQHE AKIKSLTEYL

$\begin{array}{llll}660 & 670 & 680 & 690\end{array}$

QNVEQKKRQL EESVDALSEE LVQLRAQEKV HEMEKEHLNK VQTANEVKQA

$\begin{array}{lllll}710 & 720 & 730 & 740 & 750\end{array}$

VEQQIQSHRE THQKQISSLR DEVEAKAKLI TDLQDQNQKM MLEQERLRVE 
760

770

780

790

800

HEKLKATDQE KSRKLHELTV MQDRREQARQ DLKGLEETVA KELQTLHNLR

810

820

830

840

850

KLFVQDLATR VKKSAEIDSD DTGGSAAQKQ KISFLENNLE QLTKVHKQLV

$\begin{array}{lllll}860 & 870 & 880 & 890 & 900\end{array}$

RDNADLRCEL PKLEKRLRAT AERVKALESA LKEAKENASR DRKRYQQEVD

$\begin{array}{lllll}910 & 920 & 930 & 940 & 950\end{array}$

RIKEAVRSKN MARRGHSAQI AKPIRPGQHP AASPTHPSAI RGGGAFVQNS

960

QPVAVRGGGG KQV

\section{Loading of the redox agent onto the kinesin}

Kinesin was expressed as described previously and listed in the Materials and Methods ${ }^{2}$. Ferrocene monocarboxylic acid-kinesin conjugates were prepared by dissolving $4 \mathrm{mg}$ ferrocene monocarboxylic acid (FCA, Fisher Scientific, USA) in $800 \mu \mathrm{L}$ of BRB80 buffer (formed from a mixture of $80 \mathrm{mM}$ piperazine-N,N'-bis(2-ethanesulfonic acid buffer, $1 \mathrm{mM}$ $\mathrm{MgCl}_{2}$ and $1 \mathrm{mM}$ ethylene glycol tetraacetic acid (EGTA), $\mathrm{pH}$ 6.8; all reagents were purchased from Fisher Scientific, USA) which contained $5 \mathrm{mM}$ biotin-sulfo-N-Hydroxy succinimide (biotin-sulfo-NHS, Sigma, USA ), 2 mM 1-Ethyl-3-(3'-dimethylaminopropyl) carbodiimide (EDC, Fisher Scientific, USA) and $10 \mu \mathrm{M}$ taxol (Fisher Scientific, USA). The mixture was subsequently incubated for $15 \mathrm{~min}$ at room temperature. Upon incubation, $90 \mu \mathrm{L}$ of $2.8 \mathrm{mg} / \mathrm{mL}$ kinesin (expressed as previously described) was mixed with the FCA solution and incubated for $4 \mathrm{~h}$ at $4{ }^{\circ} \mathrm{C}$; the reaction was terminated by adding $\beta$-mercaptoethanol (Fisher Scientific, USA, $20 \mathrm{mM}$ final concentration) ${ }^{3}$.Loading of the FCA onto the kinesin was estimated by subtracting the amount of the free FCA in the supernatant of span down FCA-kinesin conjugates from the total FCA being added in the reaction. Specifically, after the FCA-kinesin conjugates were synthesized as described above, the FCA-kinesin conjugates were span at 30,000 rpm for 10 min using an Allegra 64R centrifuge (Beckman Coulter, USA). After the centrifugation, the supernatant was collected and evaluated using fluorescence microscopy (Nikon, USA). The pellet was mixed with BRB80 again and the centrifugation was repeated until there was no kinesin visible in the fluorescence microscopy. The concentration of the free FCA in the supernatant was also evaluated using the electrochemical workstation and a user-performed calibration curve (Figure S1). 


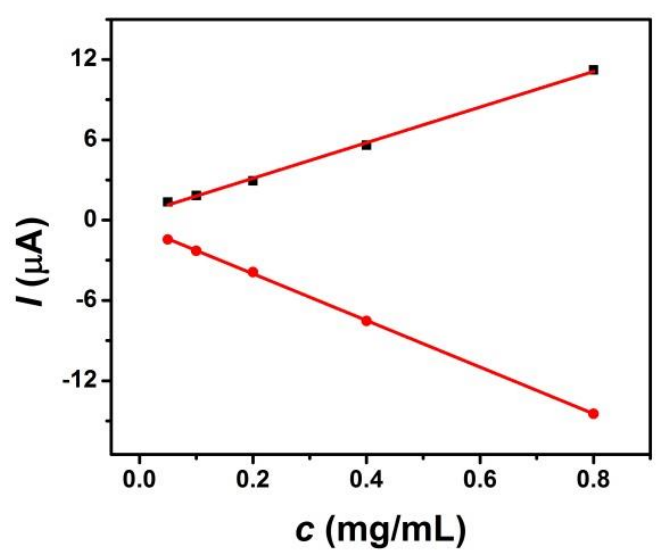

Figure S1: The calibration curve of FCA.

\section{Theoretical evaluation of FCA-kinesin conjugates Immobilized at the user-functionalized electrode interface}

Calculations considered the total length of a MT $\left(\mathrm{L}_{\mathrm{MT}}\right)$ as being the overall electrode area $\left(3.14 \mathrm{~mm}^{2}\right.$ ) divided by an individual MT diameter (i.e., $\mathrm{d}=25 \mathrm{~nm}^{4}$ ) as well as that kinesin molecules binding area is about $8 \mathrm{~nm}$ at a MT individual binding site ${ }^{5}$. Maximum theoretical numbers of FCA-kinesin conjugates $\left(\mathrm{N}_{\mathrm{FCA} \text {-kinesin-max }}\right)$ on the electrode was thus calculated using:

$$
N_{F C A-\text { Kinesin-max }}=\frac{L_{M T}}{8 n m}
$$

The full area of the electrode was defined as $A_{E}$.

$$
A_{E}=\pi(0.5 d)^{2}=3.14 \times(0.5 \times 0.02)^{2}=3.14 \times 10^{-4} \mathrm{~m}^{2}
$$

The area that the microtubule occupied on the electrode defied as $A_{M T}$ equaled its cross-section area ( $\left.\mathrm{A}_{\mathrm{MT} \text {-cross }}\right)$. When the $\mathrm{MT}$ formed a monolayer on the electrode it was considered that full surface area of the electrode $\left(A_{M T}=A_{E}\right)$ was occupied. The length of the microtubule was calculated using the area of the gold electrode divided by the diameter of the microtubule.

$$
\begin{aligned}
& L_{M T}=\frac{A_{M T}}{d_{M T}}=\frac{A_{E}}{d_{M T}}=\frac{3.14 \times 10^{-4} \mathrm{~m}^{2}}{25 \times 10^{-9} \mathrm{~m}}=1.256 \times 10^{4} \mathrm{~m} \\
& N_{F C A-\text { Kinesin-max }}=\frac{L_{M T}}{8 n m}=\frac{1.256 \times 10^{4} \mathrm{~m}}{8 \times 10^{-9} \mathrm{~m}}=1.57 \times 10^{12}
\end{aligned}
$$




\section{References:}

1 Kull, F. J., Sablin, E. P., Lau, R., Fletterick, R. J. \& Vale, R. D. Crystal structure of the kinesin motor domain reveals a structural similarity to myosin. Nature 380, 550 (1996).

$2 \mathrm{Hu}, \mathrm{X}$. et al. Biological self-assembly and recognition used to synthesize and surface guide next generation of hybrid materials. ACS applied materials \& interfaces $\mathbf{1 0}$, 28372-28381 (2018).

3 Landers, T. A., Greenberg, H. B. \& Robinson, W. S. Structure of hepatitis B Dane particle DNA and nature of the endogenous DNA polymerase reaction. Journal of virology 23, 368-376 (1977).

4 Carlson, L.-A., Shen, Q.-T., Pavlin, M. R. \& Hurley, J. H. ESCRT filaments as spiral springs. Developmental cell 35, 397-398 (2015).

5 Dong, C. \& Dinu, C. Z. Molecular trucks and complementary tracks for bionanotechnological applications. Current opinion in biotechnology 24, 612-619 (2013). 


\section{CONCLUSIONS}

\section{Applications of Cellular Components in}

\section{Engineered Environments}

Manipulation of cellular components in synthetic environment has attracted interest for applications ranging from sensors to nanelectronics, and from catalysis to biomedical devices. In such applications, biological-based processes such as specific recognition and self-assembly direct the formation of assemblies and hierarchical structures, all at minimum energy costs, with high efficiency and reliability. This thesis presents novel concepts and implementations of such cellular components in engineered environments. From studying single molecule assembly forces using atomic force microscopy approaches, to using single biomolecules for printing patterns with ultrahigh resolution, and from combining single biomolecules with user-synthesized nanomaterials to create novel self-assembled hybrids to be manipulated on user-defined surfaces for the creation of the next generation of nanocircuits, the work included herein offers viable solutions for emerging science and engineering concepts that aim to promote natural-based processes for synthetic applications. Briefly:

Chapter 1 reveals how atomic force microscopy (AFM) can be used to study the interactions between biological molecules. Specifically, we used atomic force microscopy to study and rank the energetics of the interactions between streptavidin and its ligand, namely anti-streptavidin antibody, which is used for nucleotide array, ELISA, and flow cytometry applications, just to name a few. By using the well characterized biotin-streptavidin molecular recognition as a control and by applying multi-system Gaussian fitting analysis to the distribution of the rupture forces evaluating the binding events between streptavidin and anti-streptavidin antibodies, we found that multi- and single binding events occurred at user-functionalized interfaces to account both for association and dissociation of the specific protein-ligand as well as for the structural integrity and binding efficiency of the analyzed complex. Our analysis also showed that by controlling the specificity of binding and limiting the non-specific interactions, fine tuning of the binding events can be achieved, all as a result of the packing of the proteins or ligands at their binding sites. The importance of elucidating the energetics of protein-ligand bonds is underscored by applications in a variety of fields from the fundamental science of understanding operative biomolecular interactions that 
govern and maintain structure and function in the biological world, to their synthetic translation for in situ assays in biosensors, immunoassays and diagnosis, or in pharmaceutical development. (Publication: $\underline{\mathrm{Hu}, \mathrm{Xiao}}$, and Cerasela Zoica Dinu. "Analysis of affinities between specific biological ligands using atomic force microscopy." Analyst 140.24 (2015): 8118-8126.)

Building on the work in Chapter 1 and on the knowledge of single molecule recognition binding events, in Chapter 2 we integrated our specific binding approaches for the creation of the next generation biological pen capable to print on user-functionalized surfaces single molecules with ultrahigh resolution. Advancing ultrahigh resolution (below $10 \mathrm{~nm}$ ) direct writing technologies could lead to impacts in areas as diverse as disease detection, genetic analysis and nanomanufacturing. Current methods based on electron-beam, photo- or dip-pen nano-lithography are laborious and lack flexibility when aiming to generate single molecule patterns for application specific integration. We hypothesized and demonstrated that a novel strategy could be developed to allow for writing of parallel and yet individually addressable patterns of single molecules on user-controlled surfaces. The strategy was based on using the in vitro self-recognition of tubulin protein to assembly the rigid protofilaments of a microtubule, with such microtubule to be subsequently used as a "biopen" capable of writing "inks" of single kinesin molecules in user-defined environments. In the cell, microtubules are giving structural integrity while serving as regular and uniform tracks for transport of vesicles or organelles; microtubules are growing with their plus end (fast growing end) towards the cell periphery while the slow end (or minus end) is oriented towards the cell nucleus. Kinesin uses a microtubule track in vivo to progress to specific locations with processive and coordinated $8 \mathrm{~nm}$ steps, and in vitro with speeds below $1 \mu \mathrm{m} / \mathrm{s}$, all under the transformation of the chemical cycle of adenosine triphosphate (ATP) into mechanical work and towards the fast growing end (or plus end) of the microtubule. Our results show that single kinesin inks could be written under the chemical hydrolysis of adenosine triphosphate and observed under both atomic force and optical microscopy. Upon extending inks functionalities, integration of soft and hard materials for nanostructure assembly and complex single molecule pattern formation is envisioned. (Publication: $\underline{\mathrm{Hu}, \mathrm{Xiao}}$, and Cerasela Zoica Dinu. "Bio-Pen For Direct Writing of Single Molecules on User-functionalized Surfaces" in review in Nature Communication, 2018) 
Extending the work from Chapter 1 and 2 into Chapter 3 allowed for us demonstrate hierarchical integration of biological components for the construction of the next generation of hybrid materials. Specifically, in this chapter, we synthesized a hybrid nanowire by using binding of biological molecules and their recognition processes at interfaces with synthetic materials, namely carbon nanodots. Several strategies attempted to create nanometer-scale integrated circuits to be used for optical devices by using closely spaced metallic nanodots as a possible solution to reduce energy losses at interfaces. However, such strategies do not provide the proof of concept that the scaffold-addressable nanodots forming such structures are capable to reduce energy losses or advance nanometer-scale implementation of nanowires in functional devices.

We created the next generation of bio-hybrids nanowires through the self-recognition and self-assembly properties of the biological molecules responsible for cell structure formation. Specifically, using user-synthesized sulfur-doped C-dots templates and tubulin, the precursor of microtubules cytoskeletal filaments, we demonstrated the ability to control the synthesis and stability of bio-nanowires as well as their conductivity. The physical and chemical properties (e.g., composition, morphology, diameter etc.) of such user-synthesized bio-hybrids were investigated using atomic and spectroscopic techniques, while the electron transfer rate was estimated using peak currents formed during voltammetry scanning. Our results demonstrate the ability to create individually hybrid nanowires capable to reduce energy losses; such hybrids could be used in the future for the advancement and implementation of nanometer-scale functional devices. (Publication: $\underline{\mathrm{Hu}, \mathrm{XiaO}}$, et al. "Protein self-assembly onto nanodots leads to formation of conductive bio-based hybrids." Scientific Reports 6 (2016): 38252.)

Chapter 4, takes the advantages of biological integration with the non-biological framework for hybrid formation to the next level. Specifically, we show that user-synthesized hybrid nanowires such as the ones synthesized in Chapter 3 could be manipulated in vitro under constant chemical energy of adenosine triphosphate (ATP) and have the potential to be implemented in the next generation of synthetic applications from drug delivery to diagnosis systems, and photocatalytic to optical devices. For this, we used kinesin molecular motors expressed and isolated in our lab and we demonstrated not only inherent hybrid transport activity in vitro but also our ability to implement biological function (through the use of selfassembly and biological recognition) to a non-biological material (carbon nanodots) for 
creating the next generation smart materials with extended applications as sensorial systems. (Publication: $\underline{\mathrm{Hu}, \mathrm{Xiao}}$, et al. "Biological self-assembly and recognition used to synthesize and surface guide next generation of hybrid materials." ACS Applied Materials \& Interfaces 10.34 (2018): 28372-28381. )

Lastly, in Chapter 5 we designed the next generation of sensitive biosensors based on assembly and integration of the biological components named above to detect single molecule targets with nanomolar resolution. Our strategy is based on creating sensitive electrodes capable to evaluate and detect molecular assembly formation as refined by self-assembly and self-recognition events, all under adenosine triphosphate (ATP) chemical energy control. Our analysis demonstrates not only that the proposed kinesin-MT biosensor is easy to create, fast, convenient and economic in its design and implementation, but further, that is capable to detect single binding and dissociation events. Lab-on-chip integration of such biosensor could possibly be used for signal controlling of sensitive and exquisite nanoswitches to allow storage or release of single target molecules. (Publication: $\underline{\mathrm{Hu}, \mathrm{XiaO}}$ and Cerasela Zoica Dinu. Biomolecular interfaces based on self-assembly and self-recognition form biosensors capable to record single molecule binding and release. Submitted to small) 